\title{
Realised Niche Shifts, Rapid Evolution and Phenotypic Plasticity in Introduced Plants
}

\author{
Charles Daniel Clark
}

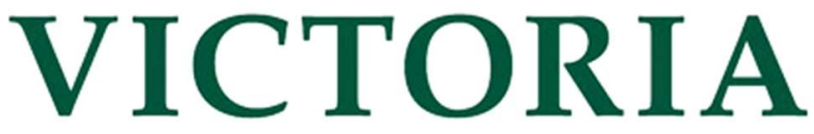

UNIVERSITY OF WELLINGTON

TE WHARE WA $\bar{A}$ ANGA

O TE ÜPOKO O TE IKA A MĀUI

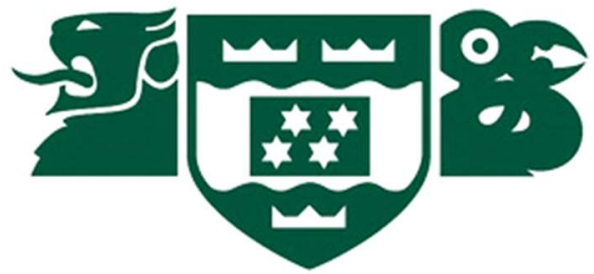

A thesis submitted to the Victoria University of Wellington in partial fulfilment to the requirements for the Degree of Doctor of Philosophy in Ecology and Biodiversity

School of Biological Sciences

Faculty of Science

Victoria University of Wellington

February 2018 



\section{Abstract}

Recent biological invasions provide a unique opportunity to examine how species may adapt to novel conditions over relatively short time frames. Introduced species may respond to novel environmental conditions in the new range via rapid evolution, phenotypic plasticity, or the rapid evolution of phenotypic plasticity. However, the prevalence of these different mechanisms in introduced species remains unclear. In this thesis, I explore how introduced plant species may adjust their phenotype when introduced to a new range.

First, I tested for evidence of phenotypic change through time in key morphological traits (plant height, leaf area, leaf shape, and leaf mass per unit area), using historic herbarium records for 34 plants introduced to Australia and New Zealand. Thirty-two out of 94 trait-species combinations showed evidence for change through time. The rate and direction of trait change was variable across species and the local climate. One possibility is that species introduced to a new range exhibit different trait responses depending on the relative difference in environment between the native and introduced range. To investigate this, I quantified climatic niche shifts in introduced species relative to their native range. I then predicted trait change through time from the magnitude and direction of climate niche shift in a meta-regression. This is the first study to simultaneously assess trait change in multiple introduced species in relation to a shift in their realised niche. Overall, climate niche shifts did not predict trait change through time, suggesting that climate may not be the predominant driver of trait change in plants introduced to Australia and New Zealand. Alternatively, the combined uncertainty and the mismatch in spatial scales that may arise when combining these two methods could mask any underlying patterns in plant trait responses to the new environment.

It has been hypothesised that introduced species may respond to a sudden change in environment, by rapidly selecting for an increase in phenotypic plasticity. I tested for a difference in phenotypic plasticity between the native and introduced ranges of a beach daisy, Arctotheca populifolia. Contrary to my expectations, A. populifolia has shown a loss of phenotypic plasticity in as little as 80 years since its introduction to Australia. When using a meta-analysis to test for an overall difference in plasticity across multiple traits, I found that the current practice of calculating an effect size of an effect size (Hedges' $d$ ) can lead to misleading results. I demonstrate how this issue arises when calculating a difference in Hedges' $d$ between two populations with different standard deviations. I propose an alternative way to calculate Hedges' $d$ to give a more accurate reflection of the difference in plasticity between ranges. 
Finally, I combine different lines of evidence from the previous chapters in a case study to explore how $A$. populifolia has changed since its introduction to Australia, and examine any discrepancies between the results. A glasshouse experiment revealed distinct trait differences between native and introduced populations of $A$. populifolia, which were not reflected in trait change through time inferred from herbarium specimens. Additionally, measured trait differences between ranges in the glasshouse experiment better reflected a niche shift into wetter climate, than the predicted trait change through time from herbarium specimens. This suggests that trait differences determined in glasshouse or common garden experiments, may be a more suitable approach to assess trait change in relation to a realised niche shift than using herbarium specimens.

Keywords: rapid evolution, phenotypic plasticity, height, leaf area, leaf shape, LMA, SDMs, niche shift, niche expansion, herbarium specimens, New Zealand, Australia, meta-analysis 


\section{Acknowledgements}

I would first to thank the Victoria University of Wellington for providing me with the opportunity to research this PhD. I would like to thank my primary supervisor, Stephen Hartley, for clear guidance, helpful ideas and interesting discussions throughout my PhD candidature. His quantitative skills and understanding of Ecology were key in the development of this thesis, and in improving on my own knowledge and abilities throughout the process. I would also like to acknowledge my secondary supervisor, Kevin Gould, for his advice in regards to plant physiology and comments to improve the thesis. I would like to thank Angela Moles for providing me with the excellent research opportunity to run an experiment at UNSW, Sydney Australia, in addition to her supervision and guidance while there. I would also like to thank Claire Brandenburger, Stephanie Creer, and the rest of the Big Ecology lab at UNSW, for the help and advice while working there. I would also like to acknowledge Joanna Buswell, for the extensive collection of plant trait data which was made available to me, contributing to a key component of my research. I am particularly grateful to the Victoria University of Wellington, the Victoria University of Wellington Faculty Strategic Research and the Centre for Biodiversity and Restoration Ecology for the provision of scholarships and grant funding that was crucial in allowing me to conduct the research for this thesis. Lastly, Lauren Bryce, for her support throughout sharing this $\mathrm{PhD}$ journey with me, and much needed proof reading at the end. 
'Nothing in evolution or ecology makes sense, except in light of the other' (Pelletier, Garant \& Hendry 2009)

Dedicated to my parents 


\section{Contents}

\section{Part I Introduction}

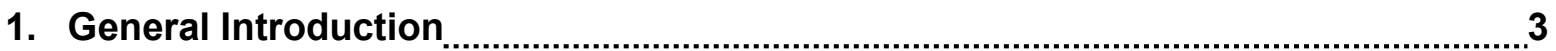

1.1. Background

1.2. Do introduced plants show evidence for trait change through time?

1.3. Do niche shifts predict trait change through time?

1.4. Does phenotypic plasticity change in the introduced range? $\ldots$

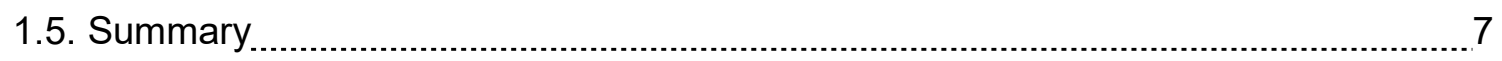

1.6. Thesis Structure

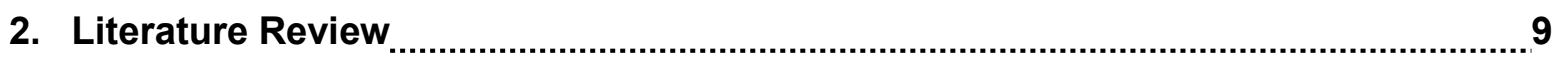

2.1. Rapid evolution in introduced Species $\ldots$

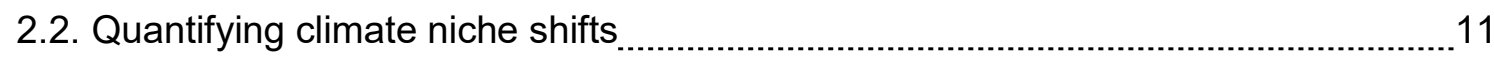

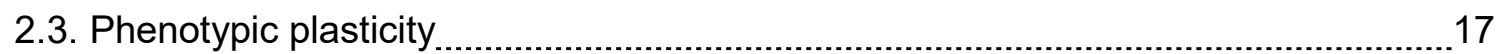

2.4. Phenotypic plasticity and evolution

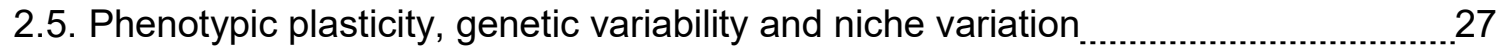

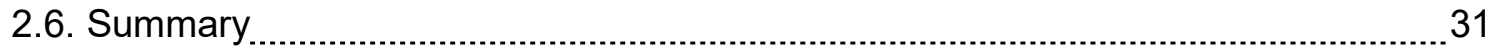

\section{Part II Data Chapters}

3. Do introduced plants show evidence for morphological trait change through time?

3.1. Introduction

3.2. Methods

3.3. Results

3.4. Discussion

3.5. Summary

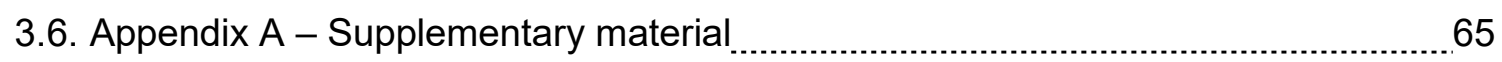

4. Do climate niche shifts predict trait change through time in introduced plants?

4.1. Introduction

4.2. Methods

4.3. Results

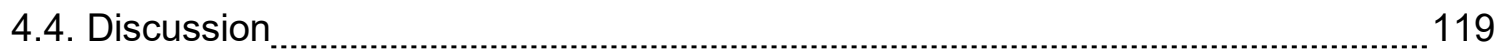

4.5. Summary

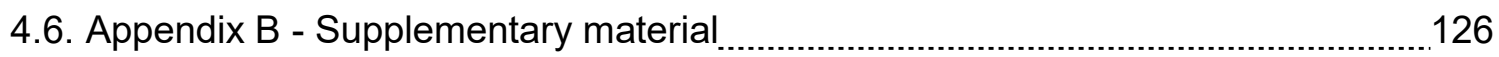


5. Rapid loss of phenotypic plasticity in the introduced range of the beach daisy, Arctotheca populifolia

5.1. Introduction

5.2. Methods

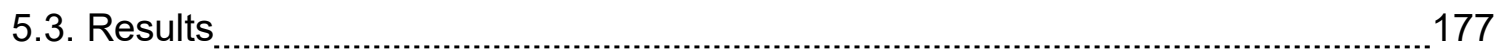

5.4. Discussion

5.5. Summary

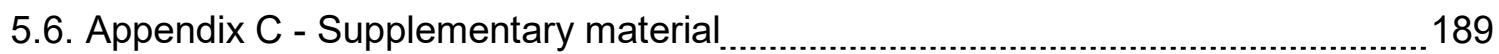

Part III Supporting Chapters

6. An effect size of an effect size: a problem with calculating a difference in plasticity between two groups $\ldots$

6.1. Rationale

6.2. Approach

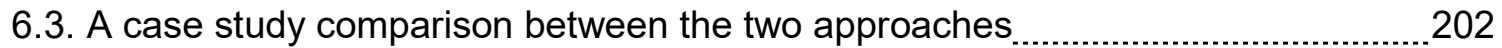

6.4. Comment on an alternative effect size index to calculate plasticity .......................206

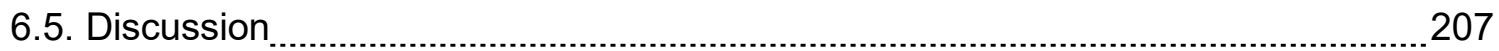

7. Do rates of trait change through time, trait differences between ranges and climate niche shifts follow similar patterns?

A case study of Arctotheca populifolia

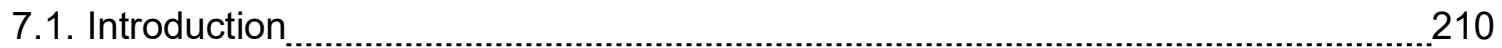

7.2. Methods

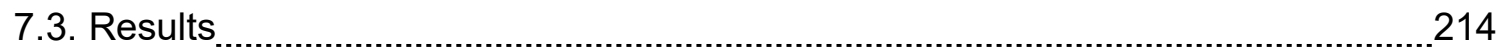

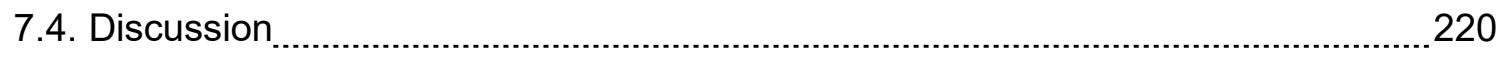

7.5. Summary

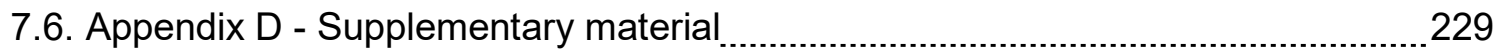

Part IV Discussion

8. Discussion

8.1. Recap of thesis findings

8.2. Research considerations

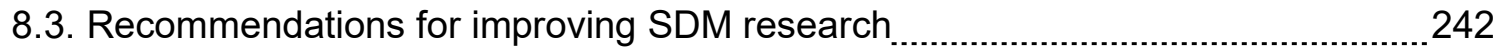

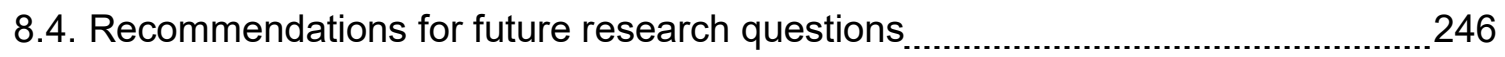

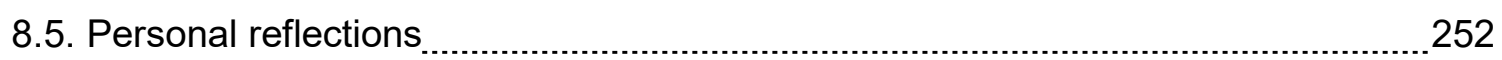

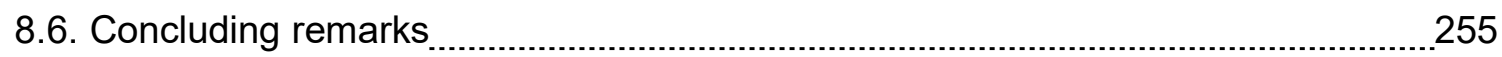

9. References 257 


\section{List of Figures}

Figure 1. Conceptual diagram of the overlap between the native range niche and introduced range niche

Figure 2.

Fundamental properties of (linear) reaction norms

Figure 3.

The potential increase in plasticity as a population adapts to a new optimal phenotype following a change in environment.

Figure 4.

The potential sources of phenotypic variability and niche breadth for individuals within three hypothetical populations

Figure 5.

A hypothetical scenario in which a realised niche shift could lead to a shift in the fundamental niche overtime

Figure 6.

PCA analysis for all 35 CliMond climate variables for

Australian and New Zealand herbarium specimens

Figure 7.

Flow diagram of methods used to collate data and address the three key questions of the study

Figure 8.

Flow diagram of the procedure for quantifying climate niche shift indices.

Figure 9.

Demonstration of the climatic niche calculated for the native and introduced ranges of Euphorbia peplus 100

Figure 10.

Categories of climate niche space. 103

Figure 11.

The calculation of niche centroid shifts in climate space 108

Figure 12.

Figure 13.

Example plot of how the magnitude and direction of a niche centroid shift would be expected to predict the rate and direction of change of a given trait through time 109

The proportional rate and direction of trait change through time for each species introduced to Australia and New Zealand

Figure 14.

Summary of mean niche shift metrics for Australia and New Zealand.

Figure 15.

The difference in the rate of trait change through time for New Zealand herbarium specimens sampled from novel climate space vs those sampled from climate space shared with the native range.

Figure 16.

The study site locations in the native South African range and the introduced Australian range.

Figure 17.

The traits measurements of Arctotheca populifolia, relative to the scale at which they were measured on the plant.

Figure 18.

Reaction norms for the South African and Australian

populations across high and low nutrient treatments. 
Figure 19.

Reaction norms for the South African and Australian populations across high and low water availability treatments 180

Figure 20. The overall difference in plasticity between the introduced Australian populations and the native South African population in the nutrient and water treatment experiments. 182

Figure 21. Hypothetical scenarios comparing two effect size calculations of the difference in plasticity $\left(P_{\text {diff }} v 1, P_{\text {diff_v2 }}\right)$ between a native and introduced population

Figure 22.

Meta-analysis for the difference in plasticity between native and introduced populations of Arctotheca populifolia when the effect size $P_{\text {diff }}$ is calculated as $\boldsymbol{P}_{\text {diff_v1 }}$ and $\boldsymbol{P}_{\text {diff_v2 }}$ 204

Figure 23. The geographic distribution and realised climate niche of Arctototheca populifolia's native and introduced range

Figure 24 .

Comparison of measured trait differences between ranges and predicted trait change through time

Figure 25. Example of how a lack of absence or pseudo absence data on one edge of a niche axis can create unreliable predicted suitability scores

Figure 26.

The hypothetical relationship between climate niche space, predicted habitat suitability and functional/fitness related traits

\section{List of Supplementary Figures}

Figure S1. Frequency histograms of the correlation coefficients for the temperature $\left({ }^{\circ} \mathrm{C}\right)$ and moisture index of each sample location with the year it was collected

Figure S2 - S8. Predictions from the best fitting GLS models which included a significant term for year, for species introduced to Australia and New Zealand.

Figure S9 - S42. Climate niche calculations and overlap for species introduced to Australia and New Zealand 130

Figure S9 - S42. Climate niche calculations and overlap for species introduced to Australia and New Zealand. 130

Figures S43 - S47. Photographs of Arctotheca populifolia as herbarium specimens, in the field, and in the glasshouse 


\section{List of Tables}

Table 1.

Table 2.

Table 3.

Table 5.

Table 6.

Table 7.

Table 8.

Table 9.

Table 10.

Table 11.

Table 12.
Key terms in plasticity research.

Summary of model combinations used to assess trait

change through time. 46

Summary of the frequency of significant terms in the best fitting model, across different traits for species introduced to Australia and New Zealand

The proportional rate of trait change through time over 100 years for species introduced to Australia. 53

The proportional rate of trait change through time over 100 years for species introduced to New Zealand 55

Summary of the frequency of trait-species combinations which included a significant term for year, in relation to the original study 56

Summary of the frequency of trait-species combinations which include a significant term for year, in the GLM and GLS analysis.

The predicted direction of trait change in response to the direction of the niche centroid shift. 107

The proportional rate of trait change through time in response to the magnitude of climate niche shift

The relationship between the magnitude and direction of the niche centroid shift and the direction and rate of trait change through time.

Arctotheca populifolia traits that show highly different effect sizes for difference in plasticity between ranges $\left(P_{\text {diff }}\right)$, when calculated as $P_{\text {diff } v 1}$ or $P_{\text {diff } v 2 \text {. }}$

Trait differences between the native source population and introduced populations of Arcotheca populifolia, in response to water availability. 


\section{List of Supplementary Tables}

Table S1. The loadings for each climate variable along the first two axes of the principle component analysis (PC1 and PC2) for herbarium specimens recorded in Australian and New Zealand

Table S2 - S8. Results of the best fitting GLS models that explained trait variation for each species introduced to Australia and New Zealand

Table S9.

Niche shift metrics used in meta-analysis to predict trait change through time, for each species introduced to Australia 126

Table S10.

Niche shift metrics used in meta-analysis to predict trait change through time, for each species introduced to New Zealand

Table S11.

The rate of trait change through time for species introduced to Australia

Table S12.

The rate of trait change through time for species introduced to New Zealand.

Table S13 - S46. Niche shift metrics for niche expansion and niche unfilling, in analog and non-analog climates. 130

Table S47.

Location of the sample sites for Arctotheca populifolia

Table S48.

The difference in phenotypic plasticity between the Australian and South African Ranges. 190

Table S49.

The phenotypic plasticity for each range, in response

to high and low nutrient and water treatments 191 


\section{List of Glossary Boxes}

Box 1.

Glossary of terms related to niche shifts 96

Box 2.

Glossary of terms related to calculating an effect size 197

\section{List of Equations}

Equation 1.

Species occupancy 101

Equation 2. Schoeners' D 101

Equation 3. GLS model formula to determine trait change through time. 104

Equation 4. Pooled standard deviation ( $\left.\mathrm{SD}_{\text {pooled }}\right)$ 175,198

Equation 5. A difference in plasticity between two groups $\left(P_{\text {diff }} v 1\right)$ 198

Equation 6. Combined standard deviation ( $\left.\mathrm{SD}_{\text {combined }}\right)$. 199

Equation 7.

A difference in plasticity between two groups $\left(P_{\text {diff }} v 2\right)$ 200

Equation 8.

Proposed model formula to predict trait differences between ranges, from a realised niche shift 250 


\section{List of Acronyms}

$\begin{array}{ll}\text { AIC } & \text { Aikaike's information criterion } \\ \text { BRT } & \text { Boosted regression tree } \\ \text { EICA } & \text { The evolution of increased competitive ability hypothesis } \\ \text { GLM } & \text { General linear model } \\ \text { GLS } & \text { General least squares model } \\ \text { MaxEnt } & \text { Maximum entropy (species distribution model) } \\ \text { PCA } & \text { Principle component analysis } \\ \text { SD } & \text { Standard deviation } \\ \text { SDM } & \text { Species distribution model }\end{array}$

\section{List of Plant Trait Acronyms}

$F_{v} / F_{m} \quad$ Plant chlorophyll fluorescence

LMA Leaf mass per unit area

LMF Leaf mass fraction; the proportion of total plant biomass as leaves

RMF Root mass fraction the proportion of total plant biomass as roots

SLA Specific leaf area

SSD Stem-specific density 
Part I

Introduction 


\section{General Introduction}

\subsection{Background}

Globalisation has led to widespread introductions of alien invasive species throughout the world (Meyerson \& Mooney 2007; Hulme 2009; Perrings et al. 2010). In recent years, there has been growing recognition of the role that rapid evolution may play in the success of species adapting to novel environmental conditions when introduced to a new range (Cox 2004; Maron et al. 2004; Prentis et al. 2008; Whitney \& Gabler 2008). Most research examining rapid evolution in introduced species, focuses on invasive species that have known adverse impacts in the introduced range (Blossey \& Notzhold 1995; Maron et al. 2004; Lavergne \& Molofky 2007). However, there are also many introduced species that become naturalised in their new range, without major direct adverse impacts. If introduced species are capable of successfully colonising and becoming established in a new range because they are especially proficient in adapting to a sudden change in environment, then they may also be particularly resilient in adapting to projected future climate change. If this is the case, then introduced species may also be better suited to cope with predicted future climate change, and thus pose an even greater threat to native species in the future.

\subsection{Do introduced plants show evidence for morphological trait change through time?}

Recent biological invasions provide a unique opportunity to examine how species may adapt to novel conditions over relatively short time scales (Hulme \& Barrett 2013). Most studies examining trait divergence in introduced species focus on single species, comparing native and introduced populations in controlled common garden environments (Maron et al. 2004; Williams et al. 2008; Ebeling et al. 2011). Recently, there has been growing evidence for morphological change through time in introduced plants, assessed from trait measures of 
historical herbarium records (Buswell et al. 2011; Flores-moreno et al. 2014; Dalrymple et al. 2015). This provides a unique approach in that: i) rates of rapid evolution in introduced species can be inferred from the change in their trait values through time, since their first documented occurrence in a new range, and ii) data is relatively accessible, inexpensive, and easy to measure, allowing for morphological change through time to be assessed simultaneously across multiple species.

Analysis of plant trait change through time can be made more informative and robust, by incorporating data on the climate and location of each herbarium specimen. Examples of rapid evolution from the analysis of herbarium specimens could potentially be masked by, or conflated with, trait variation as a species spreads across an environmental gradient through time and/or spatial non-independence of sample locations. A lack of independence in samples can occur between individuals that occur in close proximity to each other. As trait variation is likely to be driven by similar ecological processes, they do not represent truly independent samples (Hulbert 1984). This issue can be avoided by the inclusion of the georeferenced location of each sample in the analysis, to account for any potential spatial auto-correlation in the residuals of the fitted models.

Many introduced species initially colonise as small founder populations, before subsequently expanding in their new range (Moody \& Mack 1988; Mooney \& Cleland 2001; Bryers et al. 2015). It is possible that some of the introduced species in Buswell et al. (2011) expanded across an environmental gradient through time, while simultaneously exhibiting a plastic trait response along that gradient. Thus, the appearance of significant trait change through time, without controlling for environmental factors, could be misinterpreted as rapid evolutionary change. The inclusion of climate variables can allow for variation in climate conditions to be controlled for when predicting trait change through time, as well as assessing how the rate of trait change through time may vary in different climates. This will provide further insight into how specific climatic factors may be driving trait change through time, and the relative prevalence of rapid evolution in introduced species. 


\subsection{Do niche shifts predict trait change through time?}

If climate is a key driver of trait change through time in introduced populations, this could be due to climatic differences between the native and introduced ranges. In this case, the more dissimilar the climate between ranges, the greater the selective pressure to adapt towards a new optimal trait value. Correlative species distribution models (SDMs) are often used to model a species' environmental niche, based on the environmental parameters of the locations where the species is known to occur (Peterson \& Vieglais 2001; Peterson 2003; Guisan \& Thuiller 2005). By creating separate SDMs for a species' native and introduced ranges, it is possible to quantify the magnitude of the realised climate niche shift that a species has undergone when introduced to a new range (Broennimann et al. 2007; Gallagher et al. 2010; Petitpierre et al. 2012).

Species distribution models typically assume niche conservatism, meaning that a species will maintain its niche-related ancestral ecological requirements through time (Wiens \& Graham 2005), overlooking possible changes in biotic interactions and the evolutionary potential of a species. However, the evidence for niche conservatism is mixed, with different studies showing support for both niche conservatism (Peterson 2011; Petitpierre et al. 2012) and niche differentiation (Fitzpatrick et al. 2007; Broenniman et al. 2007; Gallagher et al. 2010; Tingley et al. 2014; Bocsi et al 2016). If a species does undergo a realised niche shift when introduced to a new range, this could expose them to novel environmental conditions that then drive rapid evolution (Müller-Schärer et al. 2004; Lavergne \& Molofsky 2007; Pearman et al. 2008; Gallien et al. 2016). In this case, the greater the climate mismatch between ranges (i.e., larger niche shift) then the greater the expected selective pressure to evolve in the introduced range. This idea can be tested by using the rate of trait change through time from measures of herbarium specimens to infer rapid evolution (Buswell 2009; Buswell et al. 2011). If climate is a predominant driver of trait change through time, then the rate of trait change through time may be expected to correspond with the magnitude of the realised climate niche shift. 


\subsection{Phenotypic plasticity}

In order to ensure that evidence for climatic niche shifts or trait change through time from modelled predictions is reliable, predictions should be validated using real trait measurements of plants, grown in controlled common garden environments (Vaughan \& Omerod 2003; Buswell et al. 2011; Hulme \& Barrett 2013). Recent research conducted by Brandenburger et al. (2017), compared introduced populations with the native source population for one of the species included in the herbarium specimen trait dataset (Arctotheca populifolia). They found a number of morphological traits to show significant divergence between the native and introduced populations. This suggests that Arctotheca populifolia may have undergone rapid evolution subsequent to being introduced to its new range.

Another mechanism that introduced species may employ to cope with a sudden change in environment is phenotypic plasticity. Phenotypic plasticity is the capacity for a single genotype to exhibit multiple phenotypes in response to varying environmental conditions (Bradshaw 1965; Pigliucci 2001; Whitman \& Agrawal 2009). As plasticity enables an individual to adjust its phenotype to better match the environment, it is thought to be particularly beneficial in facilitating introduced species to successfully colonise and expand into a new range (Sexton et al. 2002; Richards et al. 2006; Davidson et al. 2011).

However, rapid evolution and phenotypic plasticity should not be viewed as independent mechanisms to cope with a change in environmental conditions. Phenotypic plasticity in itself can be considered a property of the genotype, that can be selected upon and evolve (WestEberhard 2003; Lande 2009; Crispo et al. 2010). It has been hypothesised that greater phenotypic plasticity may be selected for in introduced species, broadening their niche and increasing their likelihood of survival when introduced to a novel environment (Bossdorf et al. 2005; Richards et al. 2006; Zou et al. 2009). Yet evidence for an increase in plasticity in a species' introduced range is mixed (Bossdorf et al. 2005; Williams et al. 2008; Flory et al. 2011), and the relative role that plasticity may play in benefiting a species when introduced to 
a new range remains unclear. I aim to test to test for a difference in plasticity in populations of an introduced plant (Arctotheca populifolia), relative to its native source population.

\subsection{Summary}

When introduced to a new range plant species may undergo a shift in the environmental niche they occupy. This can expose them to novel environmental conditions in which they may then adjust their phenotype through rapid evolution, phenotypic plasticity, or rapid evolution of phenotypic plasticity. I aim to explore these possibilities in my thesis by addressing the following specific questions:

1. Does the addition of climate and location data affect the prediction of trait change through time from historic herbarium records, for multiple species introduced to Australia and New Zealand? (Chapter 3).

2. Do climate niche shifts predict trait change through time in multiple species introduced to Australia and New Zealand? (Chapter 4).

3. Does phenotypic plasticity differ in populations from the introduced range of Arctotheca populifolia, relative to their native source population? (Chapter 5).

4. Is there a problem with calculating a difference in plasticity as an effect size of an effect size (Hedges' d; Davidson et al. 2011), and what would be a more suitable approach? (Chapter 6).

5. Does the predicted rate of trait change through time and climate niche shift observed in Arctotheca populifolia correspond to differences in trait values between its native and introduced range? (Chapter 7). 


\subsection{Thesis structure}

1. This thesis is divided into four key sections: part I is an introduction to the thesis (Chapters 1 - 2), part II comprises key data chapters (Chapters $3-5$ ), part III is supporting data chapters (Chapters $6-7$ ), and part IV is a general discussion of the thesis (Chapter 8).

2. The second chapter comprises a review of relevant literature, with the goal to both set the scene for the research and to introduce and explain key concepts referred to through the rest of the thesis.

3. The first three data chapters (Chapters $3-5$ ) are written in the style of individual manuscripts. Chapter 5 is written as a journal article that is planned to be submitted for publication; consequently, some information on environmental differences is omitted as it is intended to be included in another published journal article (this information is discussed in more detail in the Chapter 7).

4. Chapter 6 is written in the style of a short response paper, addressing a methodological issue in calculating an effect size from an effect size in a meta-analysis.

5. Chapter 7 is a case study that acts as a synthesis of the results from Chapters $3-5$, for the introduced species Arctotheca populifolia.

6. The final chapter (Chapter 8 ) is a discussion of the thesis as a whole: synthesising the results, discussing some of the limitations of the research, and proposing potential avenues for future research. 


\section{Literature Review}

\subsection{Rapid evolution in introduced species}

Evolutionary change has typically been viewed as a slow process occurring over very long time scales, driven by random mutations that can change in frequency due to random drift and/or through a process of natural selection (Carroll et al. 2007). However, there is growing evidence that many organisms can undergo rapid evolution over relatively short ecological timescales, often within decades (Ashley et al. 2003; Stockwell et al. 2003; Carrol et al. 2007). Many of these instances have been observed in species that have been recently introduced to a new range (Pergams \& Ashley 2001; Lee 2002; Phillips et al. 2006). The colonisation of a species to a distinct novel environment, with limited gene flow to its native source population, provides ideal conditions for rapid evolutionary change (Reznick \& Ghalmbor 2001). It has even been suggested that when an introduced species experiences novel environmental conditions in a new range, rapid evolution should be considered the norm rather than the exception (Stockwell \& Ashley 2004; Holt et al. 2005). Therefore, the recent introduction of a species to a distinct novel environment provides an opportunity to explore the prevalence and rate of rapid evolutionary change in introduced species.

It has been argued that to fully understand and detect evolutionary change, identification of the genes contributing to trait variation is necessary (Lee et al. 2014). However, Rausher and Delph (2015) argue that the identification of the underlying genes is not always required, and that evolution via natural selection can be inferred when heritable trait variation is identified to correspond to an increase in fitness. In this thesis, I explore how a change in a speciep phenotype through time or trait differences between native and introduced ranges, can imply trait change that may be a result of rapid evolution. I examine multiple species and look for trends that would be consistent with expectations when introduced to a new environment. However, without identifying genetic differences, or without a robust test for local adaptation 
via fitness differences between populations in a common garden experiment, it is not possible to conclude that evolution via natural selection has occurred. Nevertheless, if phenotypic changes that would be expected to increase fitness relative to a change in environment, are repeatedly detected in the introduced range, then it is plausible to consider that this may have been selected for via rapid evolution.

Recent research has used historic herbarium specimens to test for evidence of morphological trait change through time in introduced species to Australia and New Zealand, concluding that the observed changes in morphology were most likely due to rapid evolution (Buswell 2009; Buswell et al. 2011). This was the first multi-species study of rapid evolution in introduced plants. By taking measurements from historical herbarium specimens, they effectively used plants that were introduced up to 150 years ago as an inadvertent transplant experiment in which changes in morphology could be examined through time. The study assessed easy to measure functional plant traits related to growth and morphology (height, leaf area, leaf mass per area and leaf shape), with $70 \%$ of species showing a change in at least one trait in Australia (Buswell et al. 2011) and 33\% in New Zealand (Buswell 2009).

Morphological plant functional traits, such as height and leaf characteristics, are strongly correlated with plant growth strategy, often in relation to environmental conditions and resource availability (Grime 1977; Westoby et al. 2002; Wright et al. 2004). Thus a change in the abiotic environment following introduction to a new range would be expected to put a population under novel selection pressures that may lead to evolutionary change in these traits. Alternative explanations that could explain adaptive trait change in introduced species include the evolution of increased competitive ability (EICA) hypothesis (Blossey \& Notzold 1995) or the enemy release hypothesis (Keane \& Crawley 2002). Following a change in biotic interactions, such as the escape from herbivores present in the native range, selection will favour genotypes with improved competitive ability due to a reduced necessity to allocate resources to herbivore defences (Blossey \& Notzold 1995). However, evidence for the EICA hypothesis is mixed, with some research concluding that trait differences between ranges 
were more likely driven by abiotic factors (van Kleunen \& Schmid 2003; Felker-Quinn et al. 2013).

The direction that certain traits changed through time in Buswell (2009) and Buswell et al. (2011) was variable between species, suggesting that trait responses in the introduced range are likely to be species-specific. A possible reason for this is that the species originated from different ranges, with distinct climate niches adapted to their home conditions. Consequently the climate in the introduced range would exert differing selection pressures, potentially driving trait change through time in different directions or at different rates. If differences in climate between ranges was the predominant driver of the morphological change through time observed in Buswell (2009) and Buswell et al. (2011), then it should in theory be possible for realised climate niche shifts to predict where greater selection pressures for evolution may occur. In other words, the greater the climatic difference between the native and introduced populations, the stronger the expected selection pressure to evolve.

\subsection{Quantifying Climate Niche Shifts}

\subsubsection{Species distribution models}

The increasing availability of global species distribution data from online herbarium records (e.g., GBIF, http://www.gbif.org/) and global climate data (e.g. Hijmans et al. 2005), have made correlative SDMs an increasingly popular method for predicting a species' realised niche. Correlative SDMs aim to describe the patterns between species occurrence records and the spatial environmental data where they occur (Kearney et al. 2010; Dormann et al. 2012). These approaches can then be employed to address a range of different questions, including predicting the effect of climate change on future species distributions (Thuiller et al. 2005a; Araújo et al. 2006), identifying high suitability sites in species relocation programs (Guisan et al. 2013; Carneiro et al. 2016), predicting at risk areas for biological invasions (Hartley et al. 2006; Villemant et al. 2011) or disease transmission (Peterson 2006; 2007), or testing for niche shifts in introduced species (Fitzpatrick et al. 2007; Broennimann et al. 2007). 
While SDMs provide an effective tool for predicting suitable climate that a species may occupy, they are limited by the assumption of niche conservatism (Wiens \& Graham 2005; Pearman et al. 2008), as well as not accounting for other biotic and dispersal related factors (Pearson \& Dawson 2003; Jeschke \& Strayer 2008). Niche conservatism is the tendency for a species to maintain its niche-related ancestral ecological requirements through time (Wiens \& Graham 2005), overlooking the evolutionary potential of a species. However, as is often the case in biological invasions, species can expand beyond their predicted native range niche through niche differentiation (Fitzpatrick et al. 2007; Tingley et al. 2014). This can occur through a change in the realised niche, the fundamental niche, or both (Broennimann et al. 2007).

\subsubsection{The realised and fundamental niche}

When considering niche differentiation, it is important to understand the type of niche being referred to and how different factors may be causing it to change. Hutchinson (1957) defined the fundamental niche as the sum of environments in which a species can persist without interference from other species in order to achieve positive growth. The realised niche is often referred to as the environmental space that a species can occupy when constrained by biotic interactions (Silvertown 2004; Broennimann et al. 2007). However, a key shortcoming in predicting a species climate niche, is the assumption that all the necessary combinations of environmental factors to determine the niche limits will exist somewhere in geographic space (Ackerly 2003).

Environmental barriers that limit a species dispersal can and do have a significant influence on the niche space that a species is able to occupy in the real world (Colwell \& Rangel 2009). Jackson and Overpeck (2000) define a 'potential niche', which describes the actual distribution of a species in environmental space, as the intersecting space of the 'realised niche' with the 'realised landscape' (the combination of different environmental factors that are realised by a given landscape at a given point in time). This 'potential niche' gives a better reflection of the environmental space that a species is actually able to occupy. For the purposes of this thesis, 
the 'fundamental niche' will refer to the total range of environmental conditions that a species' physiology enables it to persist in, while the 'realised niche' is the actual environmental space occupied by a species (i.e., equivalent to the 'potential niche' defined in Jackson and Overpeck [2000]). In this thesis, I use a correlative SDM approach based on observed species occurrences, thus niche shifts will refer to a shift in the climate space occupied by a species (i.e., a shift in the realised niche).

\subsubsection{Species distribution modelling approaches}

Predictions based on correlative SDMs have been criticised for their limitations in regards to relating to the actual biology of the focal species and their transferability into novel environments (Davis et al. 1998; Vaughan \& Ormerod 2003; Dormann et al. 2012). An alternative approach is to use mechanistic SDMs, which attempt to determine the causal factors that influence a species' distribution, usually through measurements of their physiological limits or metabolic rates (Kearney \& Porter 2009). However as this requires very specific data on a species' physiological limits, it can often be an unfeasible approach for many species and/or studies. Consequently, correlative SDMs are a far more commonly used approach to model species distributions (Elith \& Leathwick 2009; Dormann et al. 2012). Kearney et al. (2010) found highly similar predictions generated from correlative and mechanistic SDMs, for the Australian greater gliders' (Petauroides volans) current distribution and predicted distribution under forecasted climate change scenarios.

Correlative SDMs can be generated from fairly simple algorithms, using presence-only data (eg., BIOCLIM, DOMAIN), or from more complex algorithms that use presence-absence data (eg., GLM, MaxEnt, BRT). As actual absence data of species occurrences is rarely recorded in accurate detail, pseudo-absences are often used as an alternative. Pseudo-absences are randomly generated from the background climate space available to that species, where there are no recorded presences (Barbet-Massin et al. 2012; Senay et al. 2013). Presence-absence models tend to have the benefit of being more precise, as they can predict more complex 
niche envelopes and be more specific in identifying the threshold climates where a species does and does not occur (Lobo et al. 2010; Senay et al. 2013). However, the quality of these models is dependent on the reliability of their input data, and can result in greatly over- or under-predicting a species' suitability in a given climate. Type II errors (false absences) can be especially problematic in these models. This can occur if pseudo-absences are generated at a given location because of a lack of presence data, describing a species as absent where it does actually occur (Lobo et al. 2010; Barbet-Massin et al. 2012). Models may also give a false impression of a species' niche if they are built from variables that do not actually limit the species.

With so many modelling approaches to choose from, it can be difficult to know which is the most appropriate to use. As different modelling algorithms can give widely varying results, one approach is to create multiple models from different algorithms and then take their average suitability or evaluation scores (Petitpierre et al. 2012; Hijmans \& Elith 2017). However, there is no universal 'best' method, and the choice for the optimal model to use will likely depend on the study species, the data available, and the overall goal of the study (Porfirio et al. 2014; Hefley \& Hooten 2016).

\subsubsection{Quantifying niches in climate space}

In order to quantify climate niche shifts, the first step is usually to determine and examine the extent of the predicted niche in climate space for each range. This aspect is often overlooked in most studies that use SDMs, with most only displaying suitability scores projected directly into geographic space. It has been suggested that predicting species responses along climate axes can be used as an effective way to visually evaluate the model predictions (Elith et al. 2005). Some studies have used SDM algorithms (e.g., GLM) to predict suitability scores into two or three dimensions of climate space, and create climate niche envelopes delineated by a suitability threshold (Hartley et al. 2006; Kikils et al. 2010). However, this approach becomes increasingly complex the more variables there are included in the model. Consequently, most 
approaches that attempt to describe a niche in climate space use ordination techniques, such as principal component analysis (PCA), to combine multiple variables into two dimensions.

\subsubsection{Quantifying species niche shifts in the introduced range}

There are a number of different approaches to test for climate niche shifts. One method is to use principal component analysis (PCA) to plot the native and introduced range niches along two dimensions in climate space, followed by a between-class analysis to test for a significant difference between them (Broennimann et al. 2007; Gallagher et al. 2010; Soberon \& Peterson 2011). An alternative method to test for a niche shift, is to create reciprocal SDMs for the native and introduced range distributions (Fitzpatrick et al. 2007; Rödder \& Lötters; 2009; Medley 2010; Hill \& Terblanche 2014). This approach involves creating separate SDMs for the native and introduced range, and then seeing how well they predict into the other range. While these approaches can indicate if a niche shift has occurred (Fitzpatrick et al. 2007), or quantify the degree of niche overlap between ranges (Broennimann et al. 2007), they do not describe the characteristics of the niche shift or account for the prevalence of climate space available in each range (Broennimann et al. 2012; Petitpierre et al. 2012). Accounting for the prevalence of climate conditions in each range prevents differences in species distributions from being interpreted as niche shifts when they are simply due to differences in the climate space available (Soberon \& Peterson 2011; Guisan et al. 2014).

A more recent approach outlined by Broennimann et al. (2012) has become an increasingly popular way to describe and quantify the magnitude of climate niche shifts (Petitpierre et al. 2012; Tingley et al. 2014; Kumar et al. 2015; Strubbe et al. 2015; Tingley et al. 2016). By applying a kernel smoothing algorithm to presence points in climate space to create a surface of occurrence densities, the climate niche can be determined while accounting for the prevalence of climate space available to that species (Broennimann et al. 2012). This approach has some key advantages when used to identify and quantify a niche shift. Firstly, the characteristics of a niche shift in climate space can be quantified in terms of niche 
expansion, the expansion into novel climate space in the introduced range; niche unfilling, the unfilling of climate space that is occupied in the native range; or niche conserved, the climate space shared between both ranges (Figure 1; Petitpierre et al. 2012; Guisan et al. 2014). Secondly, the climate space occupied can be corrected for the prevalence of climate space available in each range. This means that measures of niche overlap are not overestimated due to differences in the climate space available to a species in each range. Thirdly, niche shifts can be quantified by only including climate space that is shared between ranges, and not mistaken for expansion into climate space to which it is already pre-adapted (Petitpierre et al. 2012).

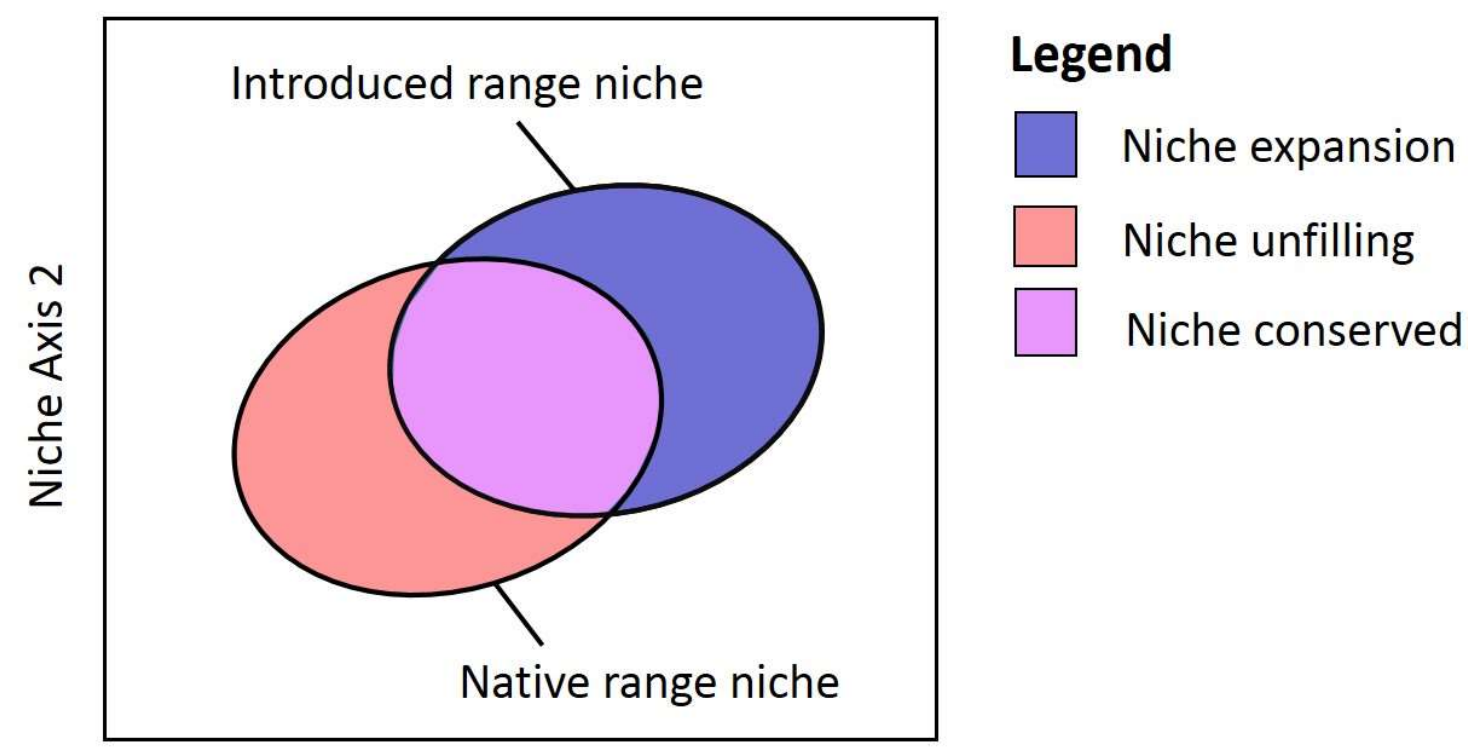

Niche Axis 1

Figure 1. Conceptual diagram of the overlap between the native range niche and introduced range niche. The overlap of predicted occurrence densities in each range allow for niche expansion, niche unfilling and niche conserved to be calculated.

Most studies that assess climate niche shifts in introduced species do so by quantifying niches in climate space along the first two axes of a principal component analysis (PCA; Broennimann et al. 2007; Petitpierre et al. 2012; Tingley et al. 2014). This has the advantage of condensing multiple explanatory variables that may explain species distributions into two key components, accounting for potential high collinearity between climate variables (Cruz-Cárdenas et al. 
2014). An alternative approach is to use a subset of bioclimatic variables to predict a species' niche (Hartley et al. 2006; Kikillus et al. 2010). These can be selected using a PCA analysis to identify climate variables that are not highly correlated, or select climate variables that are known to limit a species' distribution (Porfirio et al. 2014). The advantage of this method is that if a niche shift does occur, the direction of the shift along a climate axis can be identified and readily interpreted (i.e., a shift into wetter climate). As with other decisions that need to be made in the process of modelling climate niche shifts, the preferred axes in which to predict the species niche in climate space will likely depend on the goal of the study. If the aim is to relate climate niche shifts to a biological mechanism (i.e., trait change in the introduced range), then identifying the direction of niche shift along a specific climate axis can be more informative than using PCA axes.

\subsection{Phenotypic plasticity}

Phenotypic plasticity is the capacity for a single genotype to exhibit multiple phenotypes in response to varying environmental conditions (Bradshaw 1965; Pigliucci 2001; Whitman \& Agrawal 2009). Phenotypic plasticity can be explained as the phenotype that is expressed as a result of a genotype $\times$ environment interaction, and visualised through the use of reaction norms (Schlichting \& Pigliucci 1998; Sarkar 2004). Woltereck (1909) first introduced the concept of a reaction norm, as a function relating the different phenotypes that a genotype can express under varying environmental conditions. It is generally shown as a line or curve on a graph, that represents the change (or lack of change) of a phenotype in relation to an environmental factor or axis (Figure 2; Stearns 1989).

The reaction norm has two key properties that relate to phenotypic variation and plasticity: i) the angle of slope, which represents the degree of plasticity, and ii) the height, representing the mean phenotypic value across environments (Pigliucci 2001; Figure 2: a, b). Thus, reaction norms can describe phenotypic variation in a population of genotypes through three key characteristics: i) genetic variation, shown by a difference in the height of the focal trait 
between genotypes (Figure 2c), ii) phenotypic plasticity, if a genotype shows a change in the focal trait across environments (genotype $\times$ environment interaction), the degree of plasticity is indicated by the angle of the slope (Figure 2d); and iii) genetic variation for plasticity, indicated by a genotype $\times$ environment interaction where the angle of the reaction norm slopes are different between genotypes (Figure 2e; Pigliucci 2001).

(a)

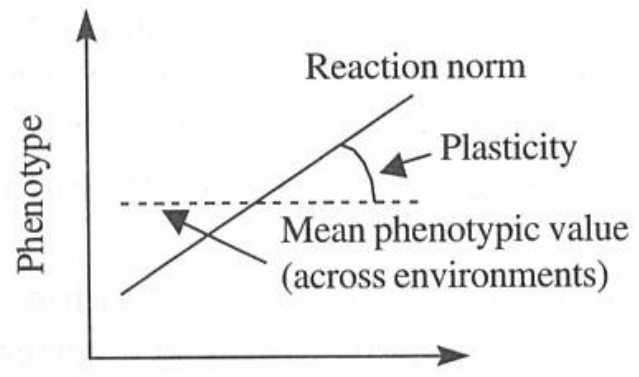

Environment

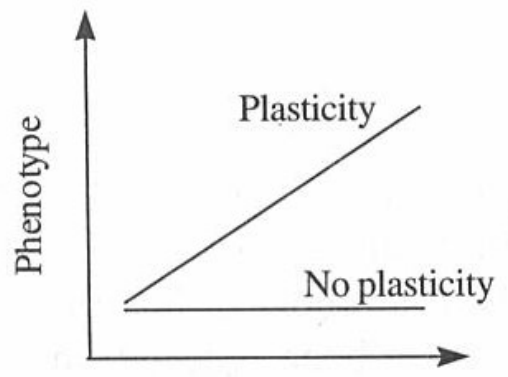

Environment

(b)

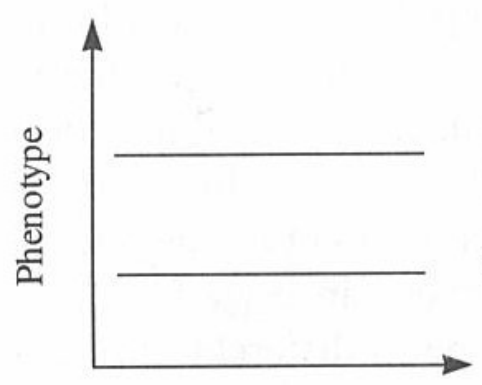

(c)

Genetic variation

No plasticity

No variation for plasticity

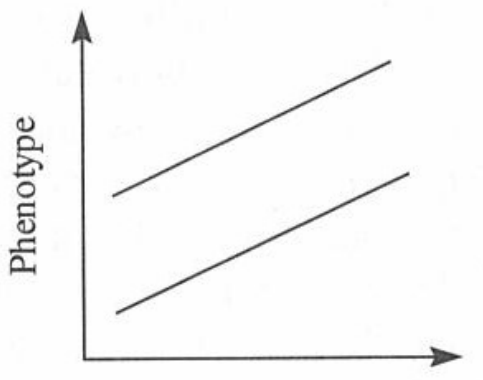

Environment

(d)

Genetic variation

Plasticity

No variation for plasticity

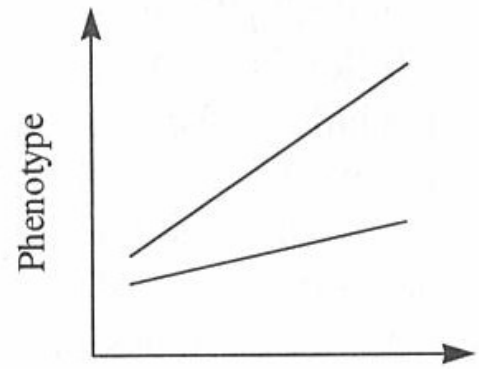

Environment

(e) Genetic variation Plasticity

Variation for plasticity

Figure 2. Fundamental properties of (linear) reaction norms: the slope (plasticity) and the height (mean phenotype across environments). a, b: the relationship between plasticity and the reaction norm; c-e: reaction norms for two individuals taken from a population, showing different genotype $\times$ environment interactions. Taken from Pigliucci (2001).

Phenotypic plasticity and reaction norms have often been used as interchangeable terms, although their meanings are not synonymous. Phenotypic plasticity is a reference to the interaction of the environment and the genotype to produce a phenotype, while the reaction norm refers to the specific form of that effect (Scheiner 1993). This can lead to some confusion as to what is meant by each term in a given context, which can then be further exacerbated 
as the definition will change depending on the scale that it is referring to; if it is at the level of individuals or populations (Table 1; Pigliucci 2005).

Table 1. Key terms in plasticity research, taken from Pigliucci (2005).

\begin{tabular}{|c|c|c|}
\hline Term & Individual level meaning & Population level meaning \\
\hline Genotype & $\begin{array}{l}\text { The actual set of genes affecting the phenotype } \\
\text { and shaping all aspects of the norm of reaction } \\
\text { (i.e., both its plasticity and 'height' in an } \\
\text { environment- phenotype space). }\end{array}$ & $\begin{array}{l}\text { Average phenotypic differences } \\
\text { among genotypes, across } \\
\text { environments. }\end{array}$ \\
\hline Plasticity & $\begin{array}{l}\text { An attribute of the individual reaction norm, } \\
\text { indicating that the genotype (through } \\
\text { interactions with the environment) generates } \\
\text { different phenotypes depending on the external } \\
\text { conditions. The only case of zero plasticity is } \\
\text { when the reaction norm is flat and parallel to the } \\
\text { environment axis. }\end{array}$ & $\begin{array}{l}\text { Average phenotypic differences } \\
\text { among environments, across } \\
\text { genotypes; a statistical measure } \\
\text { of how the across-genotype } \\
\text { phenotypic mean of a trait } \\
\text { changes with the environment. }\end{array}$ \\
\hline $\begin{array}{l}\text { Genotype x } \\
\text { Environment }\end{array}$ & $\begin{array}{l}\text { The idea that genotypes and environments } \\
\text { interact mechanistically throughout the } \\
\text { development of an organism to produce its } \\
\text { phenotype. }\end{array}$ & $\begin{array}{l}\text { Degree of non-parallelism among } \\
\text { reaction norms (which may or may } \\
\text { not be crossing), resulting in a } \\
\text { statistical } G \times E \text { effect. }\end{array}$ \\
\hline
\end{tabular}

\subsubsection{Individual phenotypic plasticity versus genetic variation}

When examining phenotypic variation it is key to consider the temporal and spatial scales that phenotypic differences may operate at. This can include short term acclimation responses, more long term irreversible responses, and the evolution of genotypes (Larcher 2003). Genetic variation at the population level, has been considered an alternative to phenotypic plasticity for dealing with environmental heterogeneity (Schlichting \& Smith 2002). There is evidence that genetic variation in plastic responses can occur both within (van Kleunen et al. 2000; Smekens \& van Tienderen 2001) and between plant populations (Leiss \& Müller-Schärer 2001; Botto \& Smith 2002). Phenotypic plasticity and genetic variability both contribute to intraspecific trait variation in heterogeneous environments (Pigliucci 2001; DeWitt \& Scheiner 2004). However, plasticity has been shown to account for more phenotypic variation in plants than genetic variation, particularly those with more exploitative resource strategies (Grassein et al. 2010). 


\subsubsection{Costs and limitations associated with phenotypic plasticity}

Phenotypic plasticity is thought to provide a fitness benefit for individuals and populations by enhancing their niche breadth (van Kleunen \& Fischer 2005). However, plasticity is also associated with a number of costs and limitations (DeWitt et al. 1998). A cost of plasticity is defined as a reduction in fitness associated with plastic genotypes relative to fixed genotypes that produce the same average phenotype across a defined range of environmental conditions (DeWitt et al. 1998; Scheiner \& Berrigan 1998). More specifically, it is the fitness cost of maintaining the genetic and cellular machinery necessary to be plastic (Scheiner 1993). Thus, if a plastic response is not necessary, it can result in overall lower fitness even when an optimal phenotype is expressed (Pigliucci 2005).

Limits to plasticity occur when a plastic response produces a sub-optimal trait in relation to its environment (DeWitt et al. 1998; Pigliucci 2005). This can happen if an organism cannot reliably detect environmental cues to express an optimal phenotype in a new environment (Moran 1992; Getty 1996; Visser 2008), potentially resulting in the expression of maladaptive phenotypes (Tufto 2000; Weinig 2000). Additionally, limits can occur if there is a lag-time in sensing and responding to an environmental cue in order to produce the optimal phenotype at the right time (Moran 1992; Stomp et al. 2008). This could arise as a result of climatic and seasonal differences when introduced to a new range. Consequently, limits to plasticity can pose problems for introduced species that experience novel environmental conditions in their new range.

\subsubsection{Phenotypic Plasticity and Introduced Species}

Phenotypic plasticity is thought to provide important benefits in facilitating the colonisation and subsequent expansion of invasive species into a new environment (Baker 1965; Nicotra 2010). It is thought to be particularly important in plants, as they are static entities and consequently have developed a number of physiological and morphological response mechanisms in order to cope with any changes in environmental conditions (Bradshaw 1965). Richards et al. (2006) 
outlined three primary scenarios in which phenotypic plasticity may enhance an invasive plant's success: 1) a Jack-of-all-trades, in which plasticity allows the invader to maintain fitness across a broad range of environmental conditions; 2) a Master-of-some, in which plasticity allows an invader to take advantage of favourable conditions, but cannot maintain fitness across a broad environmental niche; or 3) a Jack-and-master, as a combination of two. If possible, it is important to differentiate between these scenarios, in order to understand what may be driving potential differences in plasticity.

There are two main hypotheses that explain why phenotypic plasticity could be higher in introduced plant species: i) that invasive species are pre-adapted to be more plastic than noninvasive species (Williams et al. 1995; McDowell 2002; Lamarque et al. 2013); and ii) that populations introduced into a new range evolve greater plasticity than those that remain in their native range (Kaufman \& Smouse 2001; Sexton et al. 2002). The second hypothesis follows the idea that if there is genetic variation for plasticity, then it can be selected upon in the new environment (Richards et al. 2006; Crispo et al. 2010). Alternatively, it has also been suggested that phenotypic plasticity may actually facilitate rapid evolution in introduced species by allowing them to initially tolerate a new environment, and then subsequently adapt to it (Pigliucci 2001; Schlichting 2004; Lande 2009).

Buswell et al. (2011) concluded that trait change through time inferred from herbarium specimens was most likely to be attributed to rapid evolution, but were unable to rule out phenotypic plasticity as an alternative explanation. In reality, the mechanisms influencing these two processes are highly complex, with a range of interacting effects (Schlichting \& Smith 2002; van Kleunen \& Fisher 2005; Ghalambor et al. 2007; Wund 2012). Fundamentally, it is key to understand that a change in the environment will play two essential roles in evolution; initially influencing how genetic variation will translate into individual phenotypic variation, and then selecting among those phenotypes within a population (Gilbert \& Epel 2009; Wund 2012). 


\subsection{Phenotypic plasticity and evolution}

In the past, phenotypic plasticity has often been considered as nuisance, adding unwanted 'noise' in evolutionary studies (McWilliams et al. 1997; Fitzpatrick 2012; Forsman 2015). However, it is now regarded a key component in ecological and evolutionary processes (Pigliucci 2005; Whitman \& Agrawal 2009). Bradshaw (1965) recognised that plasticity itself could be a property of the genotype, and therefore also subject to selection pressure. Since then, there have been great advances in understanding the interacting effects that phenotypic plasticity can have in influencing evolution, and vice versa (Schlichting 1986; Sultan 1987; Scheiner 1993). As both plasticity and rapid evolution are regarded as key processes that can confer an advantage in a species when introduced to a new range, it is crucial to understand how prevalent these processes are and how they may interact and influence one another.

\subsubsection{Pre-adapted phenotypic plasticity.}

When introduced to a new range, phenotypic plasticity is thought to play a key role in enabling a colonising species to initially tolerate the novel environment, and then subsequently adapt to it (Pigliucci 2001; Schlichting 2004; Lande 2009). This is suspected to be particularly important in newly introduced populations, which tend to be small and may suffer from demographic problems such as low genetic variation and a predominance for drift over natural selection, limiting adaptive evolution from operating until a viable population size is established (Pigliucci 2001; Schlichting 2004). In this case, plasticity would help to buy enough time for mutation and recombination to occur, leading to evolutionary adaptation in the new environment (Wund 2012). This is a possible reason why a lag-phase is often observed between the time of initial establishment, and the subsequent expansion and occurrence of any ecological impacts associated with a species invasion (Crooks \& Soulé 1999; Lambrinos 2004; Pigliucci et al. 2006). 


\subsubsection{The Evolution of Phenotypic Plasticity}

It is evident that phenotypic plasticity can play a key role in both facilitating and impeding adaptive evolution (Pfennig et al. 2010; Wund et al. 2012). As a plastic response can directly influence the fitness of an individual, it follows that it can be selected upon and evolve (Crispo et al. 2010). Conceptually, plasticity can be considered as a trait in its own right, with the capacity for genetic variation for plasticity to occur both within and between populations (Via 1984). However, this can be confusing as plasticity is dependent on the expression of other traits, and has been argued to not be a specific property of a genotype, but rather is related to the response of a trait or group of traits (Schlichting 1986; Scheiner 1993).

\subsubsection{Evolution of Phenotypic Plasticity in response to the Environment}

A study carried out by Svanbäck \& Schluter (2012) indicated that plasticity had evolved in a marine stickleback following introduction to a new environment, allowing it to occupy a broader niche. Other empirical studies have shown populations of introduced plant species to evolve greater plasticity in biomass and leaf traits, relative to their native range (Chun et al. 2007; Bossdorf et al. 2008; Zou et al. 2009). A meta-analysis carried out by Bossdorf et al. (2005) found an increase in plasticity in introduced plant populations, in comparison to their native conspecifics for 5 out of 10 studies, with the remainder showing no significant change.

Schlichting and Smith (2002) describe a number of factors that may select for an increase in phenotypic plasticity, following a change in the environment. Central among these is the idea that plasticity will be beneficial and selected for in environments where a broader population niche is favoured (West-Eberhard 1989; Robinson \& Wilson 1994). This follows the general hypothesis that phenotypic plasticity will be favoured in unstable environments (Stearns 1989; Scheiner 1993), as an alternative to fixed phenotypes which are thought to be preferred in more stable environments (Hori 1993; Smith 1993). Thus, an increase in plasticity is more likely to be selected for in more heterogeneous environments, with increased rates of disturbance and fluctuations on small temporal or spatial scales (Padilla \& Adolph 1996; Alpert 
\& Simms 2002; Stomp et al. 2008). Plasticity has also been associated with being beneficial in more competitive, resource-high environments, as the ability to rapidly respond to changes in resource availability and adapt to environmental variation can greatly enhance competitive ability (Valladares et al. 2002; Grassein et al. 2010). On the other hand, stressful environments may select for more canalised phenotypes, as genotypes adapt to specialise in and tolerate these harsh conditions (Lortie \& Aarsen 1996; Grassein et al. 2010).

An evolutionary gain in plasticity may be selected for indirectly following a change in environment. If more plastic individuals are capable of expressing the most extreme phenotypes that best match the environment, then it will be these more plastic individuals that will be selected for in a population (Via et al. 1995). This could potentially lead to the rapid evolution of phenotypic plasticity, through a selective sweep that rapidly reduces the standing genetic variation for plasticity (Crispo et al. 2010). In this case, an already plastic "generalpurpose genotype" would have a greater fitness advantage upon introduction, which is then selected for within a population (Richards et al. 2006). Alternatively, plasticity could evolve to increase in a population following colonisation, if there is adequate genetic variation for plasticity within the population for natural selection to act upon (Bossdorf et al. 2005).

\subsubsection{Evolution of increased plasticity range}

Gomez-Mestre \& Giovani (2013) created a heuristic model that showed phenotypic plasticity to be rapidly selected for following a sudden change in environmental conditions, allowing the population to persist through a number of generations as the genotypes evolve towards a new phenotypic optimum. Once the new optimal phenotype was reached in a stable environment, plasticity was rapidly reduced due to the additional fitness costs of maintaining it, as the new phenotype became genetically assimilated. This indicates how an adaptive increase in plasticity following an introduction to a new environment can improve the match of the phenotype to the environment, increasing population viability and allowing directional selection to occur (Ghalambor et al. 2007). It also shows how phenotypic plasticity can facilitate a shift 
in a population across an adaptive valley, moving it closer to an optimal phenotype in the new environment.

Certain trait types will have different responses to selective pressures, and consequently exhibit different rates of evolutionary change (Crispo et al. 2010). This can occur if some traits are able to respond at a faster rate due to having more rapid and reliable environmental cues (Padilla \& Adolph 1996; Stomp et al. 2008). Additionally, the degree of plasticity that certain traits can express will influence their selection (Crispo et al. 2010), as well as their heritability including any genetic trade-offs that may occur between traits under different selection pressures. In general, selection for plasticity is thought to occur more frequently and at a faster rate in labile traits, such as acclimation through physiological responses, rather than irreversible changes in morphological traits (Alpert \& Simms 2002; Gabriel et al. 2005), as the former tend to be more efficient at responding to environmental cues. However, it is important to consider that plasticity across a suite of labile physiological traits (e.g., cycling of gases, photosynthetic rate), will also be likely influence the expression of fixed, longer-term morphological traits.

A possible mechanism for the adaptation to a more plastic phenotype could be through the rapid evolution of labile traits that are immediately under strong and direct selection pressures in the new environment, while maintaining certain ecological requirements for the native range through traits that are not under as strong a selective pressure to evolve. In this case, phenotypic plasticity is not evolving due to direct selection pressure to be beneficial in the new environment, but indirectly due to different evolutionary rates or genetic correlations across a suite of traits (van Kleunen \& Fisher 2005). This interaction of different selection pressures across a suite of traits could act to temporarily increase phenotypic plasticity (and niche width), until the optimal combination of trait values is reached. As the plasticity itself is not adaptive, it would then be selected against through genetic assimilation once the new phenotypic optimum is reached (Figure 3.). 

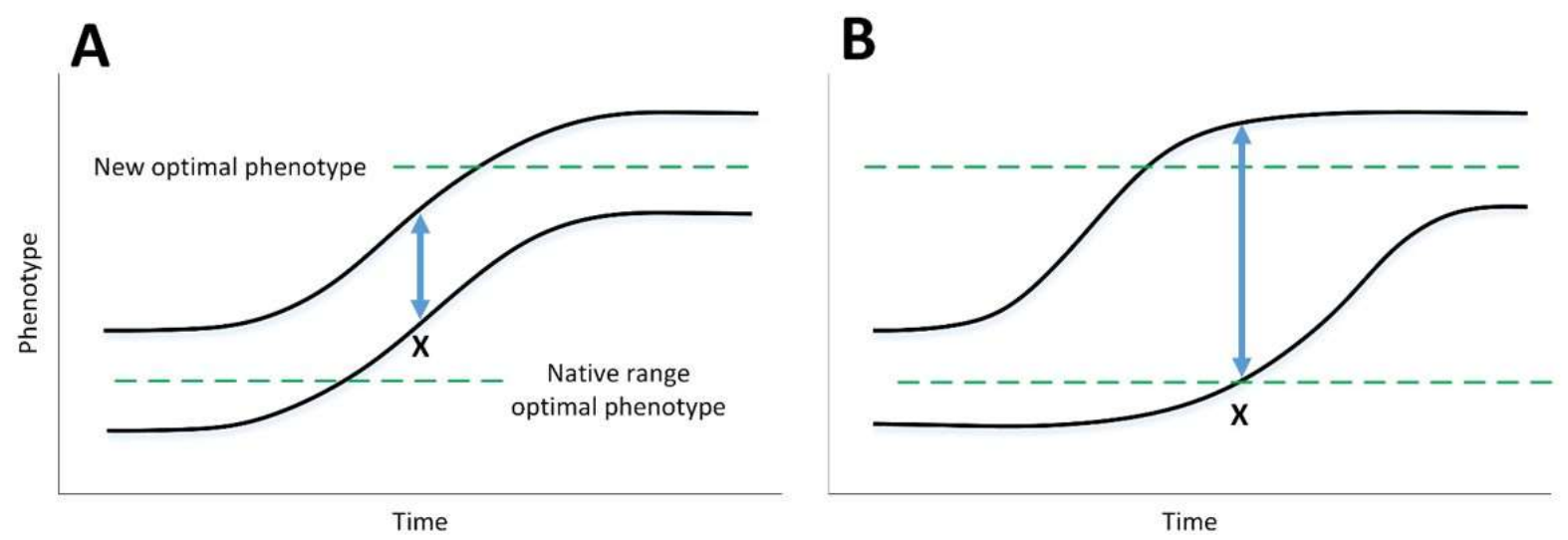

Figure 3. The potential temporary increase in phenotypic plasticity as a population adapts to a new phenotypic optimum following a change in environment. The dashed green lines show the optimal phenotype in the native and introduced ranges; the blue arrow $(\mathbf{X})$ shows the potential phenotypic plasticity of an individual at the same point in time for scenarios $A$ and $B$. A shows a scenario where there is linear evolution towards a new phenotype, with no change in the degree of plasticity through time. B shows a scenario where evolution to an optimal phenotype involves a temporary increase in plasticity. This could occur directly through selection for an increase in plasticity, or indirectly due to genetic correlations between traits or specific traits evolving at different rates.

In order for plasticity to be adaptive, it must place populations close enough to a new phenotypic optimum for directional selection to act, by suitably enhancing fitness (Ghalambor 2007). For this to happen, plasticity must provide an overall fitness advantage in the new environment, which will outweigh any associated costs in maintaining plasticity or limitations to expressing the optimal phenotype (DeWitt et al. 1998). When considering the costs of plasticity, the hypothesised evolution of increased competitive ability (EICA) and escape from natural enemies in the introduced range may make it easier for genotypes to maintain or even increase their plasticity, due to the reduced impact on fitness from natural enemies (Huang et al. 2015). This follows the idea that in the absence of herbivores, selection will favour genotypes with greater competitive ability due to reduced resource allocation to herbivore defence (Blossey \& Notzold 1995). 


\section{$\underline{2.5 \text { Phenotypic plasticity, genetic variability and niche variation }}$}

The niche width of a population is determined by the degree of phenotypic plasticity and genetic variability of individuals within a population (Bradshaw 1984; Joshi et al. 2001). This determines the range of environmental conditions in which that population can maintain a positive growth rate. Accordingly, phenotypic plasticity and genetic variability can interact to influence niche variation at the level of: i) individuals within a population, and ii) populations that make up a species' global distribution. These different scales of niche breadth can be considered in a conceptually similar way. Where populations (or groups of populations) have both high phenotypic plasticity and high genetic variation, their aggregated niche will be even broader (Figure 4). This concept could be utilised to identify phenotypic variability and niche breadth at the nested scale of individuals, populations, and species, in order to better understand the underlying causes of their variation.

The 'niche variation hypothesis' proposed by Van Valen (1965) suggests that populations with a broad niche should be more phenotypically variable than those with a narrower niche. This increase in phenotypic variability can be achieved through greater genetic variation (Lande 1980; Forsman 2014), phenotypic plasticity (Whitlock 1996; Svanbäck \& Schluter 2012), or through a combination of the two (Figure 4). Niche expansion at the species level is generally thought to arrive via increased variation among individuals rather than increased individual niche breadth (Bolnick et al. 2007). However, as plasticity is thought be adaptive when a broader population niche is favoured (West-Eberhard 1989; Robinson \& Wilson 1994), it may be selected for when introduced to a new range, leading to niche expansion. 
A. Phenotypic variability
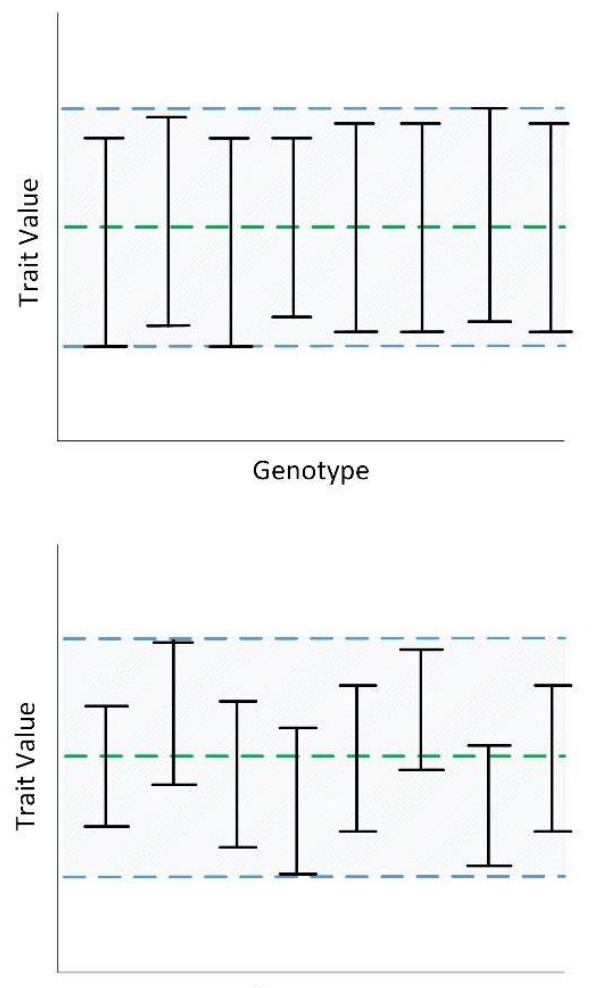

Genotype

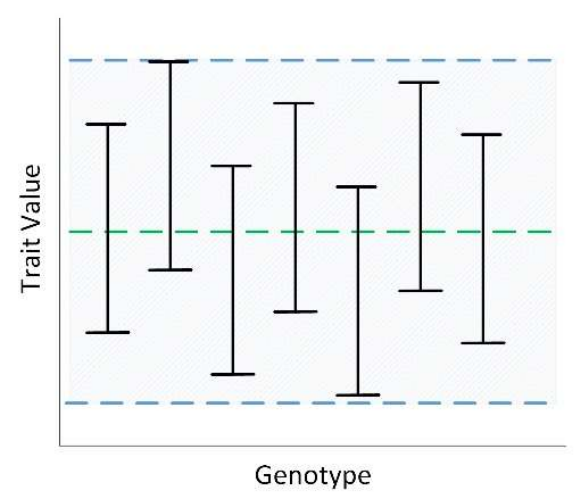

B. Niche variability

C. Source of Variation

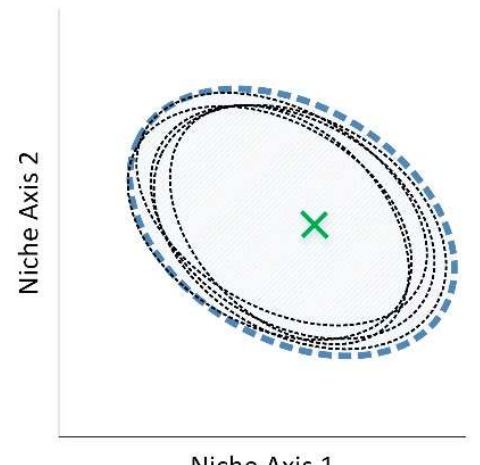

High plasticity

within individuals

Low genetic variability

between individuals

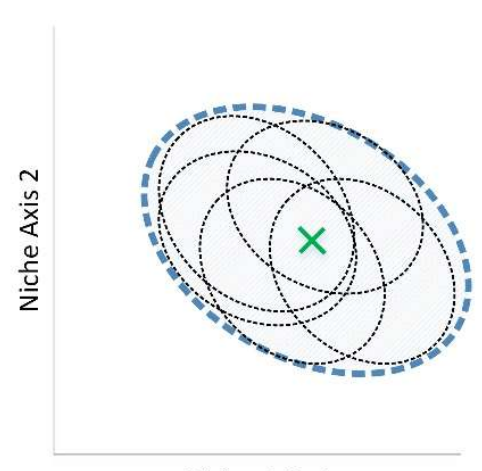

Niche Axis 1

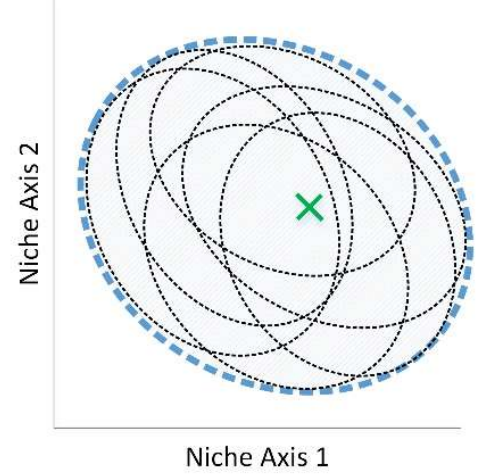

Low Plasticity

within individuals

High genetic variability between individuals

High Plasticity within individuals

High genetic variability between individuals

Figure 4. The potential sources of phenotypic variability and niche breadth for individuals within three hypothetical populations. A: The phenotypic variability of a given trait for individual genotypes within a population. The dotted green line represents the optimum trait value for that given environment. Each bar represents the potential trait plasticity for each genotype. The shaded area represents the niche width of that population as the sum of all genotypes. $\mathbf{B}$ : The niche variability for individual genotypes within a population, represented as species envelopes for 2 key niche variables. Each dotted ellipse represents the climate niche of an individual, the blue area represents the fundamental niche of that population. The green cross represents the actual climate in which the population exists, corresponding to the optimal phenotypic value (indicated by the green dotted line) in A. C: The source of phenotypic variation across the population, through individual plasticity and/or genotypic variability. 


\subsubsection{Change in the realised niche}

When colonising new environments, introduced species can change and expand beyond their predicted native range niche in various ways (Broennimann et al. 2007; Fitzpatrick et al. 2007; Tingley et al. 2014). The realised niche can shift or expand through changes in biotic interactions, such as enemy release (Keane \& Crawley 2002), that may result in the subsequent evolution of increased competitive ability (Bossdorf et al. 2005). Phenotypic plasticity can facilitate a rapid shift or expansion in the realised niche, in response to a change in biotic interactions (Berg \& Ellers 2010). High plasticity in traits related to resource uptake have been shown to enable an expansion in the realised niche of an invasive tree to Hawaii, Schinus terebinthiflorus (Stratton \& Goldstein 2001). This facilitated an increase in growth rate during the wet season while reducing water loss during the dry season, in relation to their native conspecifics.

A shift in the fundamental niche occurs through a change in environmental tolerance as a result of evolutionary processes (Broennimann et al. 2007; Tingley et al. 2014). Whereas a shift in the realised niche is often due to a change biotic or abiotic conditions that result in a change in the niche space occupied, within a species' pre-adapted fundamental niche (Soberón \& Peterson 2011; Tingely et al. 2014). Consequently, a realised niche shift is not usually associated with evolutionary adaptation (Escobar et al. 2016). However, an introduced species that shifts its realised niche will still be exposed to novel environmental conditions, which may lead to evolutionary change over time (Dlugosch \& Parker 2008; Whitney \& Gabler 2008). If a shift in the realised niche puts a population closer to its fundamental niche limit, then as it adapts to be more optimally suited in this new local environment, it may also change its environmental tolerance and expand its fundamental niche (Figure 5; Pearman 2008; Alexander \& Edwards 2010). This is suspected to be occurring in the invasion front of the common ragweed (Ambrosia artemisifolia L.), as it expands into previously unsuitable colder climates in the French Alps (Gallien et al. 2016). Thus, rapid evolution to novel environmental conditions may occur with a shift in the realised niche or the fundamental niche. However, it 
is not possible to determine if a shift in the realised niche is due to rapid evolution of physiological tolerances, or to other factors (Soberón \& Peterson 2011; Guisan et al. 2014).

\section{A: realized niche shift}

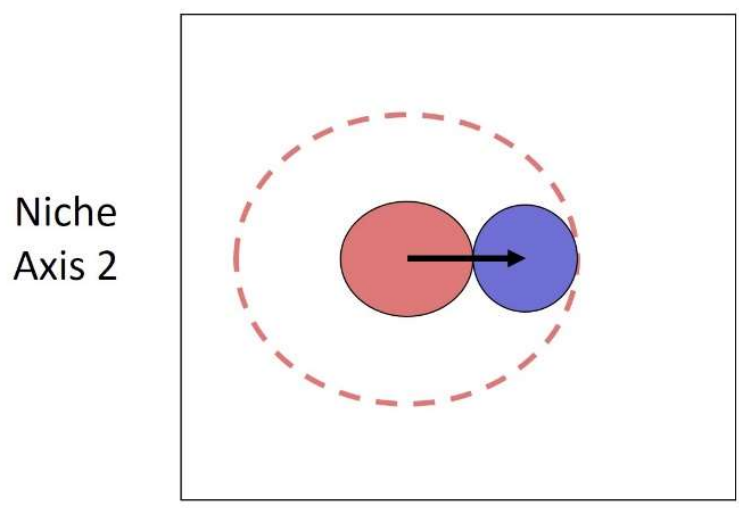

Niche Axis 1

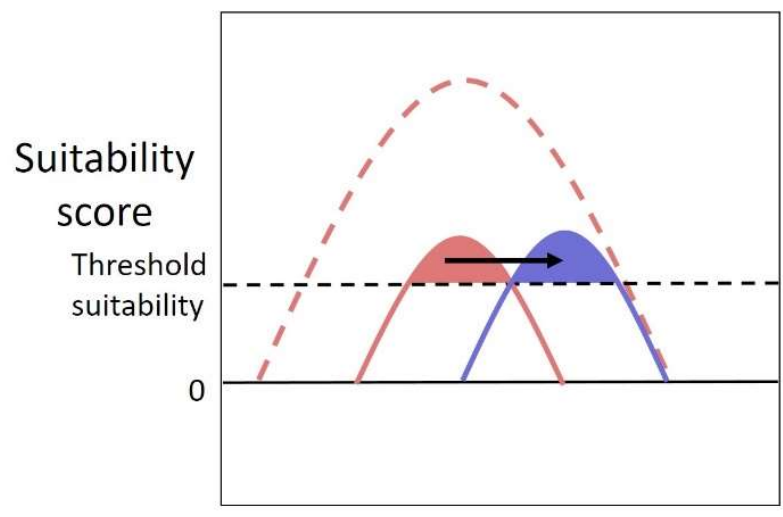

Niche Axis 1

\section{B: fundamental niche shift}

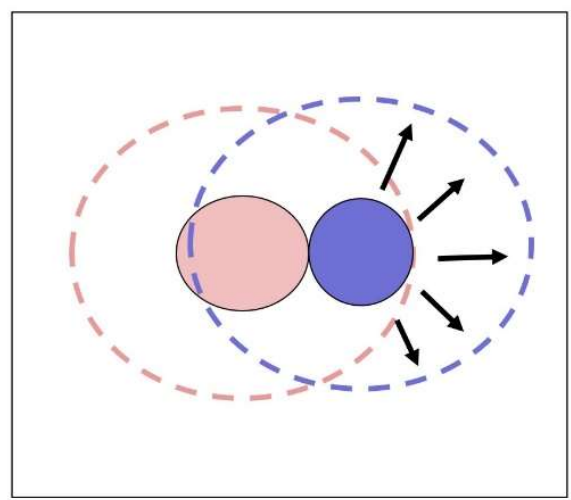

Niche Axis 1

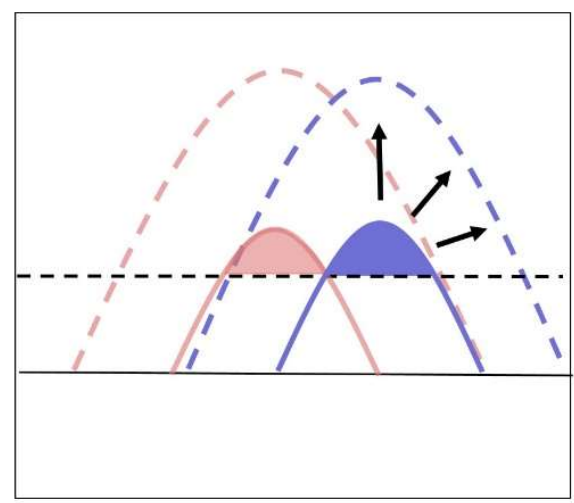

Niche Axis 1

Figure 5. A hypothetical scenario in which a realised niche shift could lead to a shift in the fundamental niche overtime. The top panels show a shift in climate envelope in 2 axes of climate space. The bottom panels show a cross section of the same niche shift, against the suitability scores used to predict the niche. Pink represents the native range and blue represents the introduced range. Solid lines/circles represent the realised niche; coloured dashed lines/circles represent the fundamental niche. The black dashed line (bottom) shows the suitability threshold, where the species can persist in its realised niche. A: an introduced species shifts its realised niche when introduced to a new range, it remains within its fundamental niche. B: as the introduced population is exposed to novel environmental conditions, it adapts to the new environment and subsequently shifts its fundamental niche limits over time. This could then lead to further expansion in the realised niche. 


\subsection{Summary}

Introduced species can often colonise and become established in a new range, with distinct environmental conditions from the native range in which they originate (Broennimann et al. 2007; Gallagher et al. 2010; Bocsi et al. 2016). However, the absolute and relative importance of phenotypic plasticity and rapid evolution, in determining an introduced species capability to persist under novel conditions in a new range remain unclear (Maron et al. 2004). In this thesis, I aim to address three key research questions: i) how often do we observe evidence for trait change through time in plant species introduced to Australia and New Zealand, ii) can climate niche shifts predict the magnitude and direction of trait change through time in introduced plants, and iii) does phenotypic plasticity differ in the introduced range populations of Arctotheca populifolia, relative to its native source population. Research in this area will improve our understanding of the capacity for non-native species to undergo evolutionary change in an introduced range, the prevalence of niche conservatism, and the usefulness of species distribution models in predicting biological invasions (Pearson \& Dawson 2003; Jeschke \& Strayer 2008). 
Part II

Data Chapters 


\title{
3. Do introduced plants show evidence for morphological trait change through time?
}

\begin{abstract}
Non-native species can often show evidence for a shift in trait values when introduced to a new range. Records of herbarium specimens that document a species since colonisation provide a unique opportunity to examine how plant traits may change through time when introduced to a new range. This study aims to test if evidence of trait change through time can be detected from historic herbarium records for 34 plant species recently introduced to Australia and New Zealand. I re-analysed change through time in key plant morphological traits (height, leaf area, leaf shape, and leaf mass per unit area) for approximately 4000 herbarium specimens, while accounting for the climate and location of each sample. Thirtytwo out of 94 trait-species combinations showed evidence for trait change through time in their best fitting model, with 19 of these detected as a significant interaction of year $\times$ climate. This suggests that trait change through time was occurring at different rates and directions in different climates. There was no clear pattern to the rate and direction of trait change through time across different traits or species, indicating that these responses are likely to be speciesspecific. It is possible that species originating from separate native ranges with distinct climates, may show differing trait response through time when introduced to the new range. Further work is recommended to explore climatic differences between the native and introduced range of these species.
\end{abstract}




\section{$\underline{3.1 \text { Introduction }}$}

There is growing evidence for plants to show rapid morphological change following their successful introduction to a new environment (Maron et al. 2004; Bossdorf et al. 2005; Buswell et al. 2011; Dalrymple et al. 2015). This could occur through rapid evolution towards new optimum trait values when exposed to novel selection pressures in the introduced range (Maron et al. 2004; Müller-Schärer \& Steinger 2004; Lavergne \& Molofsky 2007). Recent biological invasions provide a unique opportunity to examine how rapidly adaptation may occur in the introduced range (Hulme and Barrett 2013). This study aims to examine the incidence and magnitude of trait change through time for 34 recently introduced plants to Australia and New Zealand.

Plant morphological traits are strongly correlated with a plant's growth strategy, often in relation to environmental conditions and resource availability (Grime 1977; Westoby et al. 2002; Wright et al. 2004). Dry environments are often associated with shorter plants, with smaller and narrower leaves, while plants in wetter conditions tend to be taller, with larger and wider leaves (Westoby et al. 2002; Wright et al. 2004; Moles et al. 2009). Thus, a change in the abiotic environment following introduction to a new range could put a population under novel selection pressures, leading to rapid evolutionary change in these traits.

There are numerous cases of introduced species exhibiting trait variation along environmental gradients, which is generally attributed to phenotypic plasticity (Novak 2007; Colautti et al. 2009). However, genetic adaptation to climate conditions can also vary along environmental gradients (Hoffman \& Sgrò 2011; Still et al. 2005). Consequently, climate-niche evolution can occur at different rates, in response to variation in selection pressure along climate gradients (Lawson \& Weir 2014). In some cases, this can then facilitate range expansion into novel environments in the introduced range (Zenni et al. 2014; Gallien et al. 2016). If climate is a predominant driver of trait change in the introduced range, then the rate of trait change through time may be expected to occur at different rates in different climates. 
Recent research examined morphological change in plant traits through time for 41 introduced species to Australia and New Zealand, concluding that the observed changes in morphology were most likely to be due to rapid evolution (Buswell 2009; Buswell et al. 2011). This was the first multi-species study of rapid evolution in introduced plants. By taking measurements from historical herbarium specimens, they effectively used plants that were introduced up to 150 years ago as an inadvertent transplant experiment in which changes in morphology could be examined through time. The study assessed easy-to-measure plant functional traits related to plant growth and morphology (plant height, leaf area, leaf shape, and leaf mass per area), with $70 \%$ of species showing a change in at least one trait in Australia (Buswell et al. 2011) and $33 \%$ in New Zealand (Buswell 2009).

Although rapid evolution was suggested as the most likely explanation for the morphological changes observed, it is possible these changes could also be attributed to phenotypic plasticity in cases where a species spreads across an environmental gradient through time. By reanalysing the herbarium specimen data of Buswell (2009) and Buswell et al. (2011), with the inclusion of climate and location data, it should be possible to detect if trait variation is actually due to plasticity being expressed across a climatic gradient. Additionally, the inclusion of climate data could potentially reveal an interaction of the rate of trait change through time with climate, indicating that trait change through time occurred at different rates along climate gradients.

Plant height is an important trait in relation to a plant's growth strategy, and is often correlated with its competitive ability, seed mass and time to maturity (Westoby et al. 2002; Moles et al. 2009). Height can also be indicative of a plant's ability to compete for light and of its metabolic rate (Enquist et al. 1998). Plants are frequently thought to shift towards an increase in height when introduced to new range (Crawley 1987; Thebaud \& Simberloff 2001; Blumenthal \& Hufbauer 2007), which is often associated with increased invasiveness in the introduced species (Pyšek \& Richardson 2008). This increase in height is often attributed to the enemy 
release hypothesis (Keane \& Crawley 2002), or the Evolution of Increased Competitive Ability (EICA) hypothesis (Blossey \& Notzhold 1995).

The EICA hypothesis proposes that if an introduced plant population escapes from specialist herbivores in its home range, then it should evolve towards an increase in competitive ability due to a reduced necessity to allocate resources to herbivory defence. Bossdorf et al. (2005) performed a meta-analysis investigating the EICA hypothesis, with mixed results in support of it. While most studies examining changes in height in introduced species are tests of the EICA hypothesis, few examine the effect that changes in abiotic (or climatic) pressures may have on phenotype selection. Chun et al. (2007) found an increase in plant height in invasive populations of Lythrum salicaria, which was associated with a shift to higher water level conditions. Whereas no difference in stem height or growth rates was found between native and invasive populations of Sapium sebiferum (Zou et al. 2007).

Leaf size and shape play key roles in plants' resource acquisition, leaf longevity, temperature regulation, photosynthetic rate and herbivore defence (Westoby et al. 2002; Wright et al. 2004; Nicotra et al. 2011). The size and shape of a leaf affect its thermoregulation, and is strongly influenced by temperature and water availability (Nicotra 2011). Smaller and narrower leaves are often associated with hot and dry environments (Fonseca et al. 2000; Mcdonald et al. 2003; Peppe et al. 2011; Guerin et al. 2012). Maron et al. (2004) found no difference in leaf area between native and introduced populations of St. Johns Wort (Hypericum perforatum). However, introduced populations did show evidence for genetically based trait variation along latitudinal clines, indicating evidence for local adaptation across environmental gradients in the introduced range (Maron et al. 2004; Maron et al. 2007).

Leaf mass per unit area (LMA) represents a plant's carbon investment in photosynthetic area (Lambers \& Poorter 1992; Westoby et al. 2002). It is associated with a plant's growth strategy, leaf lifespan, $\mathrm{CO}_{2}$ assimilation rates, herbivore defence and resource availability, where it tends to decrease as conditions become more favourable (Grime 1977; Westoby et al. 2002; Wright et al. 2004). Plant growth rate is negatively correlated with higher LMA (Lambers \& 
Poorter 1992; Reich et al. 1997), and consequently it is often associated with a species' competitive ability.

Comparative studies have found LMA to be significantly lower in introduced populations compared to their native congeners (Bastlova \& Kvet 2002; Zou et al. 2007), while others have found no difference between ranges (DeWalt et al. 2004; Güswell \& 2006). These studies compare populations of introduced species with their native congeners, usually hypothesizing that LMA will decrease in the introduced range as they shift towards a more competitive growth strategy (Grime 2001; Güswell et al. 2006). Alternatively, a shift in LMA could be selected for in introduced species in their new range, through a change in the abiotic environment, or in accordance with the EICA hypothesis (Blossey and Notzhold 1995). LMA has been found to correlate positively with increasing aridity (Fonseca et al. 2000, Niinemets 2001; Wright et al. 2001; Poorter et al. 2009), which is associated with adaptations that help enable continued leaf function under very dry conditions (Wright et al. 2004).

The inclusion of environmental and location data for each herbarium sample allows for spatial autocorrelation to be accounted for in the analysis. Spatial autocorrelation suggests a lack of independence between pairs of observations at given distances in space (Legendre 1993). This arises because individuals in close proximity to each other will likely express traits that are driven by a whole set of similar ecological processes, and thus are not truly independent samples with respect to environmental covariates being measured (Hurlbert 1984). Pairs of individuals close to each other are also more likely to be connected by recent gene flow. Failing to account for autocorrelation when testing hypotheses using traditional methods can lead to the over inflation of type 1 errors (false-positives) and increased likelihood of detecting "redherrings" in ecological patterns using geographic data (Legendre 1993; Lennon 2000).

Colautti et al. (2009) conducted a meta-analysis of 32 common garden studies, which showed evidence for rapid evolution to be common in introduced species (evidence for phenotypic differences between native and introduced ranges). However, when latitude was included in the analysis it significantly altered the results, reversing the direction of overall trait change 
between ranges. This highlights the importance of taking into account geographic clines and potential environmental gradients when attempting to account for rapid evolution in an introduced species' range. If the significant trait change through time detected in Buswell (2009) and Buswell et al. (2011) was actually due to spatial non-independence of samples, or plasticity as a species spread across an environmental gradient, then the effect of time would be expected to no longer be significant with the addition of climate and location data.

Although evidence for trait divergence has been documented in introduced species, this is often in single species comparisons of native and introduced populations, grown in controlled environmental conditions (Maron et al. 2004; Williams et al. 2008; Ebeling et al. 2011). Thus the relative prevalence of rapid evolution across multiple introduced species remains relatively uncertain. Additionally, patterns in the rate of trait change through time can be overlooked. Historic herbarium specimens have been utilised as a tool to assess trait change through time in multiple introduced species simultaneously (Buswell et al. 2011; Flores-moreno et al. 2014; Dalyrymple et al. 2015). However, the approach used in these studies does not account for potential spatial-autocorrelation between herbarium samples, and may have allowed for the misinterpretation of phenotypic plasticity as a species spreads across a gradient through time as rapid evolution. By re-analysing the herbarium specimen data of Buswell (2009) and Buswell et al. (2011) with the inclusion of climate and location data, it should be possible to better detect the prevalence of trait change through time in multiple introduced species, if trait change through time occurs at different rates in different climates, or alternatively, if trait variation is more likely due to plasticity being expressed across a climatic gradient.

The overall aim of this study is to ask: i) how frequently do introduced species show evidence for trait change through time; ii) what proportion of plant trait-species combinations show evidence for trait variation to be best explained by: a) trait change through time, b) trait change through time occurring at different rates in different climate, c) plasticity as a species spread across a gradient, or d) no trait change; and 3) how does the addition of climate and location 
data affect the results of the analysis, compared to the original regression analysis used in Buswell (2009) and Buswell et al. (2011). 


\subsection{Methods}

\subsubsection{Collection of trait data}

Trait data were compiled from herbarium specimens for 34 species introduced to Australia and/or New Zealand, measured in Buswell (2009) and Buswell et al. (2011). The traits measured comprised plant height, leaf area, leaf shape and leaf mass per unit area (hereafter referred to as LMA). Data for these traits was collected by Joanna Buswell (Buswell 2009; Buswell et al. 2011), using following methods.

Plant height for each species was measured using a tape measure as either i) the distance between where the roots began and the top of the highest leaf, or ii) for prostate plants, height was measured as the 'stretch length' of the longest stem, from the roots to the tip of the furthest fully expanded leaf (as in Cornelissen et al. 2003). The method used to measure height was kept consistent within each species. Leaf area was calculated as the one-sided surface area of a fully expanded leaf, excluding the petiole (Cornelissen et al. 2003). Leaf shape was measured using digital callipers as the ratio of leaf width to leaf length, excluding the petiole. LMA was measured for only five species, as it requires destructive sampling of each herbarium specimen. LMA was calculated as the oven dry weight of a leaf divided by its area (including the petiole). These traits were selected as they are relatively easy to measure and reflect plant strategies for growth and resource acquisition (Westoby et al. 2002).

The species in this dataset were initially selected to be those that were introduced to Australia or New Zealand before 1920, had an annual or short-lived perennial life history, and had an average size that would fit well within an A3 size herbarium sheet (Buswell 2009; Buswell et al. 2011). Species that may have been cultivated or introduced on purpose were avoided where possible (Buswell 2009; Buswell et al. 2011). In addition to these requirements, I applied a further criteria that each species was required to have a minimum of 25 intact geo-referenced specimens to be included in the analysis. 


\subsubsection{Geo-referencing of sample locations}

I georeferenced herbarium specimens using the information available on each herbarium record sheet: either as coordinates or as a written description of the location. Where both coordinates and a description were given, they were cross referenced to check that they matched. In cases where they did not match the written description was used preferentially. Google Earth (Google Earth 2014), was used to locate written descriptions and assign them coordinate values. Herbarium specimens were omitted from the study if they did not have information on location, or if this was not precise enough to assign its location within a $20 \mathrm{~km}$ radius. Coastal records that fell outside the climate grid cells (i.e., those on small islands) were assigned the value of the closest cell on land, up to a maximum of $20 \mathrm{~km}$ away. Thus, the final dataset comprised a subset of the herbarium samples used in the original study (Buswell 2009; Buswell et al. 2011).

\subsubsection{Climate data}

Climate data for 35 bioclimatic variables, at 10' spatial resolution, was obtained from the CliMond dataset (V1.2, Kriticos et al. 2012). The first 19 'core' bioclimatic variables are derived from weekly measures of minimum temperature $\left({ }^{\circ} \mathrm{C}\right)$, maximum temperature $\left({ }^{\circ} \mathrm{C}\right)$ and precipitation $(\mathrm{mm})$, variables taken from a network of meteorological stations. The remaining 16 bioclimatic variables are derived from modelled monthly values of soil moisture index and solar radiation $(\mathrm{W} m-2 \mathrm{~d}-1)$. These are combined to give mean climate values collected over 30 years, from 1961 to 1990 , centred on 1975. Climate data was extracted for each georeferenced herbarium specimen using the Raster package in v 3.2.1 (R Core Team 2013).

To avoid over-fitting models, it was decided that they should only include terms with combinations of up to two climatic variables, which were considered to potentially be strong predictors of plant morphological change through time. The climate variables were selected based on their known potential to influence plant growth, based on their relationship to different primary climatic factors (i.e., a climate variable relating to water availability and a climate 
variable for temperature). A principal component analysis (PCA) was performed in order to select two variables that i) captured a large amount of variance across the first 2 axis of the PCA, ii) were perceived to have differing effects on plant growth.

The first two axis of the PCA analysis explained $72.7 \%$ of the total variation in climate for Australian herbarium records, and $73.7 \%$ for New Zealand records (Table S1). Through the PCA (Figure 6), I elected to use the mean soil moisture index of the driest three months of the year (PCA axis 1; Bio33, hereafter referred to as Moisture), and the mean temperature of the warmest quarter (PCA axis 2; Bio10, hereafter referred to as Temperature; ${ }^{\circ} \mathrm{C}$ ). Soil moisture index of the driest quarter of the year indicates the mean water available to a plant when it is most likely to experience drought stressed. Mean temperature of the warmest quarter indicates the mean temperature that a plant will experience during the hotter parts of the year that may limit or help to facilitate plant growth. The soil moisture index ranges from zero (oven dry) to one (field capacity), values greater than one are possible and represent excess moisture above field capacity (Kriticos et al. 2012). Although other climate datasets are available at smaller resolutions (Hijmans 2005), the CliMond dataset was preferentially used as it included climate variables that would unavailable in the other databases (i.e., soil moisture index, solar radiation). 

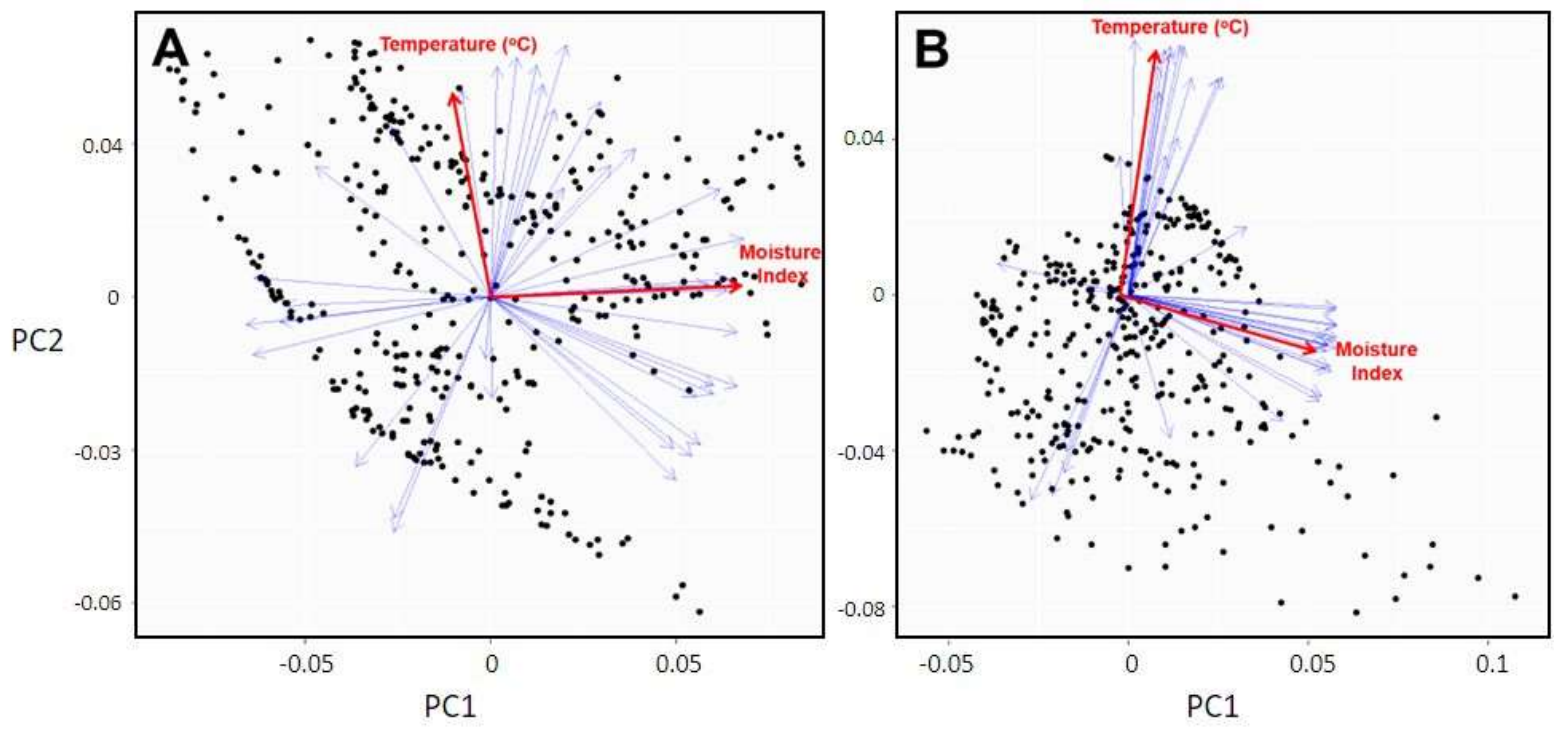

Figure 6. Principal component analysis (PCA) for all 35 CliMond climate variables for $\mathbf{A}$ ) Australia and B) New Zealand herbarium records. Highlighted in red are the two climate variables selected as climate response variables for further analysis: i) the temperature of the warmest quarter (temperature) and, ii) the moisture index of the driest quarter (moisture index).

\subsubsection{Data analysis}

Trait variation was predicted from models that included a combination of terms for year, climate (moisture and/or temperature), and geo-referenced location. All models included a term for year. I then added terms for moisture, temperature, and then terms for their interaction with year (Table 2). Analyses were performed with a spatial regression using a generalized least squares model (GLS), in the nlme package in $R$ v 3.2.1 ( $R$ Core Team 2013). The GLS is a form of linear regression, in which spatial auto-correlation between samples can be accounted for by including their locations (as geographic coordinates) in the model as a random effect. A GLS thus provides a more robust estimate of beta-coefficients and their associated p-values, by effectively down-weighting the influence of 'semi-pseudoreplicated' samples. The dependency between coordinate locations was modelled as correlogram with a Gaussian structure (Box et al. 1996; Venables \& Ripley 2002). 
Multiple model specifications were compared as I wanted to examine how trait variation was predicted by main effects for: i) year on its own, ii) how this would change with the addition of climate (a potential effect of plasticity), and iii) if there was a significant year $\times$ climate interaction (differing rates of change through time in different climates). Model terms were added sequentially, and the best fitting model selected using Akaike's information criterion (AIC; Akaike 1973). The delta AIC ( $\triangle$ AIC) was then calculated by subtracting the AIC of the best fitting model, with the AIC of the null model (a model with ' 1 ' as its only main effect). This allows a comparison of how well the combined terms in the best fitting explain trait variation in relation to the null hypothesis. Models were interpreted as having sufficient explanatory power relative to the null model if they had a $\Delta$ AIC $>2$ (Burnham \& Anderson 2002, p. 73; McGill 2015).

Table 2. Summary of model combinations used to assess trait change through time. Specifically, each term that was included in general linear model (GLM) or a general least squares model (GLS), as either a main effect or random effect.

\begin{tabular}{llll}
\hline $\begin{array}{l}\text { Model aims to } \\
\text { test: }\end{array}$ & Model & Main effect terms & $\begin{array}{l}\text { Random } \\
\text { effects }\end{array}$ \\
\hline $\begin{array}{l}\text { Comparison with } \\
\text { original study }\end{array}$ & GLM & $\begin{array}{l}\text { Year } \\
\text { Year + Region } \\
(\text { Year + Region) }\end{array}$ & none \\
\hline Null Model & GLS & 1 & Coordinates \\
\hline Year & GLS & Year & Coordinates \\
\hline Year + Climate & GLS & $\begin{array}{l}\text { Year + Temperature } \\
\text { Year + Moisture } \\
\text { Year + Temperature }+ \text { Moisture }\end{array}$ & Coordinates \\
\hline $\begin{array}{l}\text { Interaction of } \\
\text { Year } \times \text { Climate }\end{array}$ & GLS & $\begin{array}{l}\text { (Year + Temperature })^{2} \\
(\text { Year + Moisture })^{2} \\
(\text { Year + Temperature })^{2}+\text { Moisture } \\
(\text { Year + Moisture })^{2}+\text { Temperature } \\
(\text { Year + Temperature })^{2}+(\text { Year }+ \text { Moisture })^{2} \\
(\text { Year + Temperature }+ \text { Moisture })^{2}\end{array}$ & Coordinates \\
\hline
\end{tabular}




\subsubsection{Interpreting results}

Each trait measurement was $\log _{10}$ transformed prior to analysis, to satisfy assumptions of data normality and so that the proportional rate of change in a trait through time could be compared across different species. The beta coefficient $(B)$ of the year term in the model output was interpreted as the rate of trait change through time. Following the analysis, the beta-coefficient was back transformed and multiplied by $100\left(10^{\wedge(B \times 100)}\right)$, to represent a proportional rate of change through time over 100 years. The rate of change was given over 100 years as this was deemed to be more meaningful than a rate of change per year. Thus, a value of 2 would indicate a doubling in that trait over 100 years, while a values 0.5 would indicate a halving over 100 years.

If climate is a predominant driver of trait change through time in the introduced range, then the rate of change through time may be expected to occur at different rates in different climates (indicated by a significant year $\mathrm{x}$ climate interaction). The detection of a significant year $\times$ climate interaction in the best fitting model adds an additional complication when interpreting an overall rate of change. To explore how the rate of trait change through time varied in different climates, the slope of the year term $(B)$ was predicted from the model while keeping the lower quartile (Q1), the median (Q2), and the upper quartile (Q3) values of that climate variable constant. The lower quartile, median and upper quartile were calculated for the climate conditions encompassed by the herbarium specimens for each species. If terms for two climate variables were included in the best fitting model, then the same approach was used while keeping the other climate variable constant at its mean value. 


\subsubsection{Comparison of results to previous analysis}

In addition to the GLS analysis described above, I repeated the analysis used in Buswell et al. (2011) by fitting a general linear model (GLM) for each trait against terms for year, region and their interaction, for the updated dataset of georeferenced herbarium specimens. This was carried out to: i) see if the slightly reduced dataset of geo-referenced herbarium specimens gave the same results as Buswell (2009) and Buswell et al. (2011), and ii) to see if the addition of geographic coordinates and/or climate changed the effect of year in the model.

If a plant trait showed a significant change through time when using a GLM (Buswell 2009; Buswell et al. 2011), but was no longer significant when analysed with a GLS (with the inclusion of geographic coordinates and/or climate), this was interpreted in two ways: i) if year was no longer significant then this indicates that the previous analysis (Buswell 2009; Buswell et al. 2011) likely reached an overconfident conclusion of the significance of trait change through time, based on pseudo-replicated data; ii) if a previously significant effect of year was replaced by a significant effect for climate, this suggests that the conclusion of this being due to rapid evolution is more plausibly explained by phenotypic plasticity as that species spread across a climate gradient through time. To test this, a Pearson's correlation coefficient was used to test for a relationship between year and the climate of sites occupied by that species.

\subsubsection{Weighting of samples}

Each herbarium plant specimen was considered as an individual in the analysis. Where multiple leaf measurements were made on one plant, these were averaged to give a single value for the plant. Some herbarium sheets had multiple plant specimens, collected at the same time and place. In cases where multiple measurements were made from a single herbarium sheet, these were still assessed as individuals, but were weighted in the analysis according to the number of specimens on the sheet. Individuals from herbarium sheets with one specimen were giving a weight of one, while the individuals from a sheet with two specimens were each given a weight of 0.5 , and so on. 


\subsection{Results}

\subsubsection{Summary of significant terms in the best fitting model}

In total, trait change through time was assessed for 34 species, across 4 different traits, for introductions into two separate ranges. This gave a total of 94 trait-species combinations assessed in the analysis. There was a significant term for year in the best model of the GLS analysis for 13 out of 43 trait-species combinations in New Zealand, and for 19 out 51 trait species combinations in Australia (Table 2). Of these, there was a year $\times$ climate interaction for 11 trait-species combinations in Australia and eight in New Zealand. Year was the only significant term for six trait-species combinations in New Zealand and for three in Australia. year + climate were significant in two trait species combinations in both Australia and New Zealand. While only a significant term for climate was present in nine trait-species combinations in New Zealand and only one in Australia. There was no significant effect for year for 62 out of 94 species-trait combinations. The direction of trait change through time, and of any year $\times$ climate interactions occurred in varying directions for different species in the GLS analysis, which I will now explain in more detail (Tables 2 \& 3; Figures S2 - S7.). 
Table 3. Summary of the frequency of significant terms in the best fitting model, across different traits for species introduced to Australia and New Zealand. Best fitting models that include a significant term for year indicate evidence for trait change through time.

\begin{tabular}{|c|c|c|c|c|c|c|}
\hline Country & $\begin{array}{l}\text { Best Model } \\
\text { Terms }\end{array}$ & $\begin{array}{c}\text { Plant } \\
\text { Height }\end{array}$ & Leaf Area & Leaf Shape & LMA & Total \\
\hline \multirow{7}{*}{ Australia } & None & 13 & 9 & 8 & & 30 \\
\hline & Climate & & 4 & 5 & & 9 \\
\hline & Year & 2 & 2 & 1 & 1 & 6 \\
\hline & Year + Climate & & & 2 & & 2 \\
\hline & Year $\times$ Climate & 5 & 3 & 3 & 1 & 12 \\
\hline & $\begin{array}{l}\text { Total: best } \\
\text { models with } \\
\text { year significant }\end{array}$ & 5 & 5 & 7 & 1 & 21 \\
\hline & No. of Species & 16 & 15 & 15 & 5 & 51 \\
\hline \multirow{7}{*}{$\begin{array}{l}\text { New } \\
\text { Zealand }\end{array}$} & None & 10 & 10 & 9 & & 28 \\
\hline & Climate & & & 1 & & 1 \\
\hline & Year & & 1 & 2 & & 3 \\
\hline & Year + Climate & & 2 & & & 2 \\
\hline & Year $\times$ Climate & 3 & 2 & 3 & & 8 \\
\hline & $\begin{array}{l}\text { Total: best } \\
\text { models with } \\
\text { year significant }\end{array}$ & 3 & 5 & 5 & & 13 \\
\hline & No. of Species & 13 & 15 & 15 & & 43 \\
\hline
\end{tabular}




\subsubsection{Trait change through time in Australia}

Six out of 16 introduced species showed evidence for a change in plant height through time in Australia in the best fitting GLS model (Table 4; Figure S2; Table S2). Plant height showed an overall increase in Veronica arvensis to $200 \%$ of its original height over a 100 year period, while Facelis retusa decreased to $34 \%$. Four other species showed that plant height was changing at different rates or directions in different climates. Trifolium glomeratum height decreased through time in dry climates $(\mathrm{Q} 1=47 \%)$, with a reduced rate of change in wetter climates $(\mathrm{Q} 2=50 \%, \mathrm{Q} 3=106 \%)$. Euphorbia peplus showed a similar pattern of a decline in height in dry climates $(\mathrm{Q1}=67 \%)$, which then shifts to a slight increase through time in wetter climates $(\mathrm{Q} 3=124 \%)$. The relationship between the change in plant height and moisture was in the opposite direction for Pertrohagia nanteullii, with plant height increasing through time in dry climates $(\mathrm{Q} 1=158 \%)$ and decreasing in wetter climates $(\mathrm{Q} 3=34 \%)$. Plant height in Arenaria leptoclados showed a significant interaction of year with both temperature and moisture index, with height increasing at a faster rate in colder $(\mathrm{Q} 1=375 \%)$ and drier $(\mathrm{Q} 3=$ $181 \%$ ) areas, but decreasing through time when the climate was warmer ( $Q 3=181 \%$ and wetter $(Q 3=50 \%)$.

Five out of 15 introduced species showed evidence for a change in leaf area through time in Australia (Table 4; Figure S3; Table S3). Leaf area showed an overall decrease through time over a 100 year period for Cicendia quadrangularis (50\%) and Polycarpon tetraphyllum (62\%). Four other species indicated that leaf area was changing through time at different rates in different climate. Leaf area showed an increase through time in cold climates for Arenaria leptoclados $(\mathrm{Q} 1=259 \%)$ and Geranium molle $(\mathrm{Q} 1=343 \%)$, but then a decrease through time with increasing temperature. Medicago lupulina showed a decrease in leaf area in wetter climates $(\mathrm{Q} 3=75 \%$, along with an increase through time with decreasing moisture $(\mathrm{Q} 1=$ $189 \%, \mathrm{Q} 2=121 \%$.

Six out of 15 species introduced to Australia showed evidence for a change in leaf shape through time in Australia (Table 4; Figure S4; Table S4). Leaf shape showed an overall 
increase through time in Veronica arvensis (209\%) and Geranium molle (120\%), while decreasing in Gamochaeta americana (74\%). Five other species indicated that leaf area was changing through time at different rates in different climate. Arctotheca populifolia and Euphorbia peplus showed a decrease in leaf shape through time in cold climates $(\mathrm{Q} 1=66 \%$; $\mathrm{Q} 1=64 \%)$. As temperature increased the rate of change slowed for $E$. peplus (Q2 $=92 \%, \mathrm{Q}$ $=103 \%$ ) and changed direction to increase through time in A. populifolia (Q2 = 96\%, Q3 = $183 \%$ ). Cicendia quadrangularis showed a significant interaction of year with both temperature and moisture index, with leaf shape decreasing at a faster rate in warmer ( $Q 3=55 \%$ ) and wetter $(\mathrm{Q} 3=70 \%)$ climates.

Two out of five species showed evidence for change through time in LMA (Table 4; Figure S5; Table S5). Trifolium dubium showed an increase in LMA by $158 \%$ over 100 years, across all climates. Euphorbia peplus showed varying rates of change through time in LMA, through a significant interaction of year with moisture Index. Leaf mass per area increased through time in drier climates $(\mathrm{Q} 1=158 \%, \mathrm{Q} 2=116 \%)$, but decreased through time in wetter climates $(\mathrm{Q} 3$ $=70 \%$. 
Table 4. The proportional rate of trait change through time over 100 years for species introduced to Australia, predicted from the best fitting models that included a significant effect for year. When the best fitting model included an interaction with moisture or temperature, that climate was divided into low, medium and high values and the slope for year was calculated in that climate. Climate values were determined as the lower, middle and upper quartile for the climate values encompassed by those herbarium specimens. When two climate variables were included in the model, one of the climate variables was controlled for at its mean value in order to predict the other. The proportional rate of trait change through time was calculated by back transforming $\log _{10}$ beta-coefficients for the year $\left(10^{(B \times 100)}\right)$. Values of 1 represent no change, 0.5 indicates a halving while 2 would indicate a doubling, over a 100 year period. Acronyms: LMA, leaf mass unit area.

\begin{tabular}{|c|c|c|c|c|c|c|c|c|c|}
\hline \multirow{3}{*}{ Trait } & \multirow{3}{*}{ Species } & \multicolumn{7}{|c|}{$\begin{array}{l}\text { Proportional trait change through time over } 100 \text { years } \\
\text { in according climate }\end{array}$} & \multirow{3}{*}{$\triangle \mathrm{AIC}$} \\
\hline & & \multirow{2}{*}{$\begin{array}{c}\text { Year } \\
\text { All }\end{array}$} & \multicolumn{3}{|c|}{ Year $\times$ Temperature } & \multicolumn{3}{|c|}{ Year $\times$ Moisture } & \\
\hline & & & Low & Med & High & Low & Med & High & \\
\hline Height & A. leptoclados & & 3.75 & 0.96 & 0.34 & 1.81 & 1.31 & 0.50 & 12.63 \\
\hline Height & F. retusa & 0.34 & & & & & & & 2.32 \\
\hline Height & E. peplus & & & & & 0.67 & 0.97 & 1.24 & 1.79 \\
\hline Height & P. nanteullii & & & & & 1.58 & 1.11 & 0.34 & 1.18 \\
\hline Height & T. glomeratum & & & & & 0.47 & 0.50 & 1.06 & 69.48 \\
\hline Height & V. arvensis & 2 & & & & & & & 5.8 \\
\hline Leaf area & A. leptoclados & & 2.59 & 1.13 & 0.45 & & & & 12.62 \\
\hline Leaf area & C. quadrangularis & 0.5 & & & & & & & 1.88 \\
\hline Leaf area & G. molle & & 3.43 & 2.64 & 0.71 & & & & 2.66 \\
\hline Leaf area & M. Iupulina & & & & & 1.89 & 1.21 & 0.74 & 3.28 \\
\hline Leaf area & P. tetraphyllum & 0.62 & & & & & & & 6.96 \\
\hline Leaf shape & A. populifolia & & 0.66 & 0.96 & 1.83 & & & & 0.23 \\
\hline Leaf shape & C. quadrangularis & & 1.03 & 0.99 & 0.55 & 1.19 & 0.88 & 0.7 & 1.44 \\
\hline Leaf shape & E. peplus & & 0.64 & 0.92 & 1.03 & & & & 3.3 \\
\hline Leaf shape & G. americana & 0.74 & & & & & & & 4.44 \\
\hline Leaf shape & G. molle & 1.2 & & & & & & & 3.8 \\
\hline Leaf shape & V. arvensis & 2.09 & & & & & & & 8.7 \\
\hline LMA & E. peplus & & & & & 1.58 & 1.16 & 0.7 & 3.5 \\
\hline LMA & T. dubium & 1.58 & & & & & & & 2.28 \\
\hline
\end{tabular}




\subsubsection{Trait change through time in New Zealand}

Plant height showed a significant change through time for three out of 12 species in New Zealand (Table 5; Figure S6; Table S6). Acaena agnipila, Plantago coronopus and Stachys arvensis showed a significant effect for the interaction of Years $\times$ Moisture Index. Plantago coronopus and Stachys arvensis both showed a slight increase in plant height through time in drier climates $(\mathrm{Q} 1=118 \% ; \mathrm{Q} 1=131 \%)$ and a shift to a decrease through time in wetter climate $(\mathrm{Q} 3=64 \% ; \mathrm{Q} 3=46 \%)$. Acaena agnipila showed a trend in the opposite direction, with plant height decreasing through time in dry climates $(\mathrm{Q} 1=25 \%)$ and increasing through time with increasing moisture $(\mathrm{Q} 2=138 \%, \mathrm{Q} 3=193 \%)$.

Leaf area showed a significant change through time for six out of 15 species in New Zealand (Table 5; Figure S7; Table S7). Medicago lupulina and Silene gallica showed an overall decrease in leaf area to $30 \%$ and $33 \%$ of their original size over 100 years, while Myosotis arvensis showed an increase in leaf area to $238 \%$. Leaf area in Stachys arvensis decreased through time at a faster rate in wet climates (Q3 = 46\%), while showing little change in drier climates $(\mathrm{Q} 2=05 \%, \mathrm{Q} 3=105 \%)$. Cerastium glomeratum showed an overall decline in leaf area through time, with a significant interaction of year with both temperature and moisture. Leaf area decreased through time at a faster rate in warmer $(Q 3=25 \%)$ and wetter $(Q 3=$ $36 \%)$ climates.

Leaf shape showed a significant change through time for five out of 15 species in New Zealand (Table 4. Figure S8; Table S8). Myositis arvensis and Silene gallica both showed an increase in shape through time to $148 \%$ and $145 \%$ respectively, over a 100 year period. There was little to no change in leaf shape through time at low temperatures for Lythrum hyssopifolia $(98 \%)$ and Medicago lupulina (94\%), but this shifted to a decrease in shape through time at an increasing rate in warmer climates (Q3 = 59\%; Q3 =61\%). Persicaria maculosa showed a significant interaction of year with both temperature and moisture index, with leaf shape decreasing at a faster rate in warmer $(Q 3=55 \%)$ and drier $(Q 1=70 \%)$ climates. 
Table 5. The proportional rate of trait change through time over 100 years for species introduced to New Zealand, predicted from the best fitting models that included a significant effect for year. When the best fitting model included an interaction with moisture or temperature, that climate was divided into low, medium and high values and the slope for year was calculated in that climate. Climate values were determined as the lower, middle and upper quartile for the climate values encompassed by those herbarium specimens. When two climate variables were included in the model, one of the climate variables was controlled for at its mean value in order to predict the other. The proportional rate of trait change through time was calculated by back transforming $\log _{10}$ beta-coefficients for the year $\left(10^{(B \times 100)}\right)$. Values of 1 represent no change, 0.5 indicates a halving while 2 would indicate a doubling, over a 100 year period.

\begin{tabular}{|c|c|c|c|c|c|c|c|c|c|}
\hline \multirow{3}{*}{ Trait } & \multirow{3}{*}{ Species } & \multicolumn{7}{|c|}{$\begin{array}{l}\text { Proportional trait change through time over } 100 \text { years } \\
\text { in according climate }\end{array}$} & \multirow{3}{*}{$\triangle \mathrm{AIC}$} \\
\hline & & \multirow{2}{*}{$\begin{array}{c}\text { Year } \\
\text { All }\end{array}$} & \multicolumn{3}{|c|}{ Year $\times$ Temperature } & \multicolumn{3}{|c|}{ Year $\times$ Moisture } & \\
\hline & & & Low & Med & High & Low & Med & High & \\
\hline Height & A. agnipila & & & & & 0.26 & 1.38 & 1.93 & 9.8 \\
\hline Height & P. coronupus & & & & & 1.18 & 0.80 & 0.64 & 7.17 \\
\hline Height & S. arvensis & & & & & 1.31 & 0.95 & 0.46 & 1.79 \\
\hline Leaf area & C. glomeratum & & 0.48 & 0.29 & 0.25 & 0.56 & 0.47 & 0.36 & 11.27 \\
\hline Leaf area & M. arvensis & 2.38 & & & & & & & 4.66 \\
\hline Leaf area & M. Iupulina & 0.3 & & & & & & & 25.15 \\
\hline Leaf area & S. arvensis & & & & & 1.05 & 0.95 & 0.46 & 3.29 \\
\hline Leaf area & S. gallica & 0.33 & & & & & & & 6.51 \\
\hline Leaf shape & L. hyssopifolia & & 0.98 & 0.69 & 0.59 & & & & 1.6 \\
\hline Leaf shape & M. arvensis & 1.48 & & & & & & & 2.3 \\
\hline Leaf shape & M. Iupulina & & 0.94 & 0.62 & 0.61 & & & & 1.31 \\
\hline Leaf shape & P. maculosa & & 1.1 & 0.28 & 0.18 & 0.4 & 0.46 & 0.6 & 12.36 \\
\hline Leaf shape & S. gallica & 1.45 & & & & & & & 13.37 \\
\hline
\end{tabular}




\subsubsection{Comparison to the original analysis}

A comparison in the frequency of detecting a significant effect of year in a GLM for each traitspecies combination, was made between the updated geo-referenced dataset and the results found in the original analysis (Buswell 2009; Buswell et al. 2011). The year term remained significant or non-significant across 80 out of 94 of the trait-species combinations (Table 6 ). A change in the significance of the year term occurred in 14 trait-species combinations in the updated dataset, 9 became significant while 5 became non-significant.

Table 6. Summary of the frequency of trait-species combinations which included a significant term for year, in relation to the original study. Comparisons are made between the results obtained from the original dataset used in in Buswell (2009) and Buswell et al. (2011), and the updated dataset (using the same modelling approach: general linear model). A tick $(\checkmark)$ indicates if a significant effect of year was detected using that dataset, while a cross $(\boldsymbol{x})$ indicates no significant effect for year. $n$ refers to the total number of herbarium measurements included in the analysis ( $n$ was unavailable for the New Zealand dataset in the original analysis [Buswell et al. 2011]).

\begin{tabular}{|c|c|l|c|}
\hline $\begin{array}{c}\text { Original } \\
\text { dataset }\end{array}$ & $\begin{array}{c}\text { Updated } \\
\text { dataset } \\
(n=7960)\end{array}$ & Description & Total \\
\hline $\mathbf{x}$ & $\mathbf{x}$ & Year not significant in any models & 73 \\
\hline$\checkmark$ & $\checkmark$ & Year remains significant in all models & 6 \\
\hline$\checkmark$ & $\mathbf{x}$ & Year no longer significant in the updated dataset. & 9 \\
\hline $\mathbf{x}$ & $\checkmark$ & Year becomes significant in the updated dataset & 5 \\
\hline- & $\mathbf{x}$ & Species not used in original study & 1 \\
\hline \multicolumn{2}{|l}{} & 94 \\
\hline
\end{tabular}




\subsubsection{Adding climate and location data to models.}

A comparison of the frequency of detecting a significant effect for year, for each trait-species combination in the geo-referenced dataset, was made between the original linear regression (GLM) approach used and the updated spatial regression (GLS), which included terms for climate and location. Re-analysis with climate and location data revealed a change to a significant effect for year in 23 of the best fitting models in the GLS. The year term remained non-significant for 56 out of 94 of the best fitting models for each trait species combination, while also remaining significant across both modelling approaches in nine instances (Table 7).

Table 7. Summary of the frequency of trait-species combinations which include a significant term for year (for the best fitting model in the GLS analysis). Comparisons are made between analysis of the updated dataset from a general linear model (GLM), and the general-least squares (GLS) regression, which includes terms for the climate and location of each herbarium specimen. A tick $(\checkmark)$ indicates if a significant effect of year was detected using that model, while a cross $(\mathbf{x})$ indicates no significant effect for year.

\begin{tabular}{|c|c|l|c|}
\hline GLM & $\begin{array}{c}\text { GLS } \\
\text { (Climate }+ \\
\text { Location) }\end{array}$ & \multicolumn{1}{|c|}{ Description } & Total \\
\hline$x$ & $x$ & Year not significant in any models & 56 \\
\hline$\checkmark$ & $\checkmark$ & Year remains significant in all models & 9 \\
\hline$x$ & $\checkmark$ & $\begin{array}{l}\text { Re-analysis with climate and coordinate data to } \\
\text { model reveals a significant effect of year. }\end{array}$ & 23 \\
\hline$\checkmark$ & $\times$ & $\begin{array}{l}\text { Re-analysis with climate data removes significant } \\
\text { effect of year. Previous significant effect of year } \\
\text { likely due to spatial auto-correlation or plasticity as } \\
\text { that species spread across an environmental } \\
\text { gradient through time. }\end{array}$ & 6 \\
\hline
\end{tabular}


Six trait-species combinations changed to having no significant effect for year, when climate and/or location data was added to the model. Two of these were for introductions to New Zealand, in which the year term for the best-fitting model for plant height in Cerastium glomeratum and leaf area for Solenogyne gunni changed to non-significant, with the addition of coordinate data in a spatial regression. In Australia, the effect of year on leaf shape in Trifolium glomeratum and height in Silene gallica also became non-significant with the inclusion of coordinate data. This is likely to be due to spatial-auto correlation in the herbarium samples for the species.

Two of the Australian trait-species combinations that showed a significant effect for year in the GLM, were then replaced by a significant effect of climate in the GLS. The effect of year on Leaf Area in Trifolium dubium became non-significant with the addition of location and a climate term for summer temperature to the model (Table S3). The best fitting model in the GLS analysis showed leaf area to increase as temperature increased $(B=0.048, p=0.005)$. A Pearson's correlation coefficient was computed to test for a relationship between year and the climate of the sites occupied by $T$. dubium was then carried out, finding that the location of the sites sampled decreased in temperature through time $(r=-0.20, \mathrm{df}=115, p=0.002)$. An originally significant effect of year on leaf area in Veronica arvensis, was also replaced by a significant effect of temperature $(B=0.030, p=0.021)$. A Pearson's correlation coefficient revealed no significant relationship between year and the climate of the sites occupied by Veronica arvensis $(r=0.067, \mathrm{df}=65, p=0.588)$. 


\subsection{Discussion}

Based on an analysis of herbarium specimens, 32 out of 94 trait-species combinations in this study showed evidence for trait change through time in the introduced range of Australia or New Zealand. However, it should be noted that in eight of these cases the best fitting model had a $\Delta \mathrm{AIC}<2$, indicating that it did not have that much more explanatory power compared to the null model. Twenty-two of these cases involved a significant interaction between year and climate. This suggests that climate may be a key factor in driving trait change through time at different rates in different climates. Previous studies have found trait differences between native and introduced ranges that were likely a result of rapid evolution in response to climate, which proceeded to facilitate further range expansion into novel climatic conditions (Colautti \& Barret 2013; Gallien et al. 2016). Colomer-Ventrua et al. (2015) found a decrease in leaf area and increase in leaf shape (leaf area:perimeter ratio) in introduced populations of Senecio pterophorus, which were likely responses to increased drought stress in the introduced range(s). Similarly, introduced populations of Centaurea diffusa grew to a larger size with larger leaves, changes that were associated with a release from abiotic stresses in the introduced North American range (Turner et al. 2013). However, while these studies report trait differences between ranges, they could not describe how these traits had changed through time.

Other studies that examine plant trait change through time in plants using herbarium specimens have found evidence for a decline in height through time in Panax quinefolius, in response to harvesting in the U.S. (McGraw 2001). They also found trait change through time to occur at different rates in different parts of the country, attributing this to different harvesting intensities or indirect environmental effects. Václavík et al. (2017) compared leaf trait measurements in herbarium specimens for two introduced species to New Zealand (Hieracium pilosella and Echium vulgare) with those from their native, northern hemisphere range. They found leaf area to be significantly lower in herbarium records from the introduced range than those from the native range, along with a significant increase in precipitation and 
UV-B radiation at the sites sampled in the introduced range (Václavík et al. 2017). They also found no evidence of a change through time in leaf hair length or density, but did not report any effect for year on leaf area.

The addition of climate and location data to the GLS model revealed a significant effect of year for 23 trait-species combinations that were previously non-significant (Buswell 2009; Buswell et al. 2011). This was either through significant interaction of year with climate, or because the addition of climate and location to the model resulted in the year term becoming significant. This suggests that controlling for potential spatial non-independence between points and climate in the model can increase the likelihood of detecting trait change through time. When trait change through time showed an interaction with year, moisture index tended to be more prevalent in the best fitting models for plant height, while models for leaf shape and leaf area more frequently included an interaction term for temperature.

\subsubsection{Physiological expectations if climate is a driver of trait change}

If climate is a key driver of trait change, then physiological considerations predict that leaf area and leaf shape (leaf width:length ratio) would typically show a decline in drier areas. Lower values in these leaf traits are associated with greater leaf temperature regulation, and higher photosynthetic water use efficiency (Parkhurst \& Loucks 1972; Fonsecca et al. 2000; Peppe et al. 2011). Leaf area decreased at a faster rate in hotter climates for some species in both Australia and New Zealand. However, three species showed contrary results to this, in which leaf area declined at a faster rate in climates with higher moisture. Medicago lupulina showed an overall decrease in leaf area in New Zealand, and a decrease in leaf area in wetter climate in Australia. As New Zealand tends to have a much wetter climate than Australia (Adamson \& Adamson 1999), this could be an adaptive response in M. lupulina showing a decrease in leaf area through time in response to increasing moisture availability in both countries. Further investigation into the climate niche space occupied by this species in New Zealand, Australia, 
and in its native range would help to further elucidate if it is a shift in climate that is driving this trait change.

There are a number of reasons why these varying patterns in the direction of trait change through time may have occurred, which I will now outline. Firstly, the direction of an adaptive trait change in a given environment may be species specific. Thuiller et al. (2009) found that in 16 alpine plant species, nine showed an increase in height with increasing habitat suitability, while three species showed a decrease. Thus, even when assuming that a shift into wetter and/or warmer climates is favourable for a plant, not all plant species will respond in the same way. It is likely that the response of a trait will also depend on other specific microsite factors and/or biotic interactions. Secondly, the climate of the native range for certain species may differ. A species that originates from a native range with a dry climate and a species that originates from a wet climate would experience a different directional change in the environment when introduced to the new range. Therefore their traits may be may be expected to change in different directions in the introduced range. Secondly, plant morphological traits tend to have a non-linear relationship with climate (Porter \& Semenov 2005; Poorter et al. 2010; Moles et al. 2014), in which traits such as plant height may decrease towards their upper and lower climatic limits (i.e., in very wet and very dry climates). If introduced species originate from separate native ranges with different climates, then they will likely be adapted for optimum growth in different climatic conditions. Thus, the introduced range climate in Australia or New Zealand could be either beneficial or stressful for different species, driving trait change through time in different directions.

It should be noted that this study only looked at a small number of morphological traits. It is possible that other plant traits may also be selected for following a change in environment when introduced to a new range. A change in climate could also result in a change in stomatal size and density (Xu \& Zhou 2008), leaf hair length and density (Václavík et al. 2017), plant water-use efficiency (Medrano et al. 2011; Linares \& Camarero 2011), total plant biomass (Hobbie \& Chappin III 1998), and biomass allocation to roots (Sexton et al. 2002; Poorter et 
al. 2009). In some instances, there may be no net change in certain morphological traits, due to plasticity or selection in other traits enabling a plant to adjust to be better suited to the new environment. In particular, short term acclimation responses and plasticity in labile physiological traits are thought to be selected for more frequently and at a faster rate, than in morphological traits (Alpert \& Simms 2002; Gabriel et al. 2005). Nevertheless, the traits used in this study are thought to proximate a range of physiological responses, and indicate a plant's growth strategy across a range of conditions (Westoby et al. 2002; Wright et al. 2004).

\subsubsection{Other abiotic or biotic factors}

The mixed results could also be due to other unaccounted for abiotic or biotic factors having a large influence on trait variation. For example, variation in soil nutrients could also occur along an environmental gradient, and have a strong influence on trait variation. Additionally, multiple selective pressures could simultaneously drive trait change in opposite directions, resulting in no net change in traits through time.

Biotic interactions could also have a strong influence on trait change and/or variation in the introduced range. Biotic effects on introduced plants are typically associated with an increase in trait values (Jakobs et al. 2004). This is typically attributed to an escape from natural enemies in the introduced range and the evolution of increased competitive ability (EICA) hypothesis (Blossey \& Notzold 1995; Keane \& Crawley 2002), as individuals are able to allocate more resources to increased growth. Most of the species in this study showed either no evidence for trait change through time, or for key size related traits (height and leaf area) to decline through time or change at different rates in different climates. This suggests for a lack of evidence for EICA in the introduced range of these species. Other research into the EICA hypothesis has found that introduced plants often show distinct traits compared to conspecifics from their native range, but that this was more likely to be driven by other abiotic differences between ranges (Kleunen \& Schmid 2003; Felker-Quin et al. 2013). However, it is possible that in some species, biotic effects in the introduced range could have a stronger 
influence on trait change through time, or interact with climate variables used in this study to give variable results.

\subsubsection{Addition of climate and location data to the analysis}

One of the limitations of the original study (Buswell 2009; Buswell et al. 2011), was the inability to determine if trait change through time actually due to phenotypic plasticity as a species spread across an environmental gradient. As a measure to account for this, I included climate and location data in the analysis for each herbarium specimen. The addition of climate and location made year no longer significant in 6 out of 94 of the trait-species combinations. The year term was no longer significant in the best fitting model in four of these instances. This suggests that in these cases, the previous analysis (Buswell 2009; Buswell et al. 2011) likely reached an overconfident conclusion as to the significance of trait change through time, based on pseudo-replicated spatial data.

In two other trait-species combinations (leaf area in Trifolium dubium and Veronica arvensis, in Australia), the significant effect of year on leaf areas was replaced by a significant effect of temperature. This would suggest that the previous detection of a significant effect of year (Buswell 2009; Buswell et al. 2011), may have been due to phenotypic plasticity as these species spread across an environmental gradient through time. Further analysis revealed a positive correlation between year and the temperature of sites occupied by $T$. dubium, confirming that this was likely the reason why year was previously significant. However in the case of Veronica arvensis, there was no significant relationship between the year term and the temperature of the sites occupied. Given this low number it seems unlikely that plasticity as a species spread across an environmental gradient through time was a predominant explanation of trait change through time. Thus, a more confident conclusion can be drawn in interpreting trait change through time as being driven by selection in the new range. However, micro-scale landscape variation in other abiotic factors, such as soil nutrients, could still drive a plastic response that results in unaccounted for trait variation. 


\subsection{Summary}

This study suggests that non-native plants can frequently show evidence for trait change through time in the introduced range. The recurrent detection of a significant year $\times$ climate interaction in the best fitting models, indicates that trait change through time is likely to occur at different rates and/or directions in climatically dissimilar areas. However, the direction of trait change through time in response to climate did not show consistent patterns across traits and species in either the New Zealand or Australian range. One reason for this is that if species originate from native ranges with different climates, they will likely show different trait responses to novel climatic conditions when introduced to a new range. By exploring mismatches between the climatic niches occupied in the native and introduced ranges, it may help to elucidate if climate is a key driver of morphological trait change through time. This will be explored further in Chapter 4 . 


\subsection{Appendix A - Supplementary material}

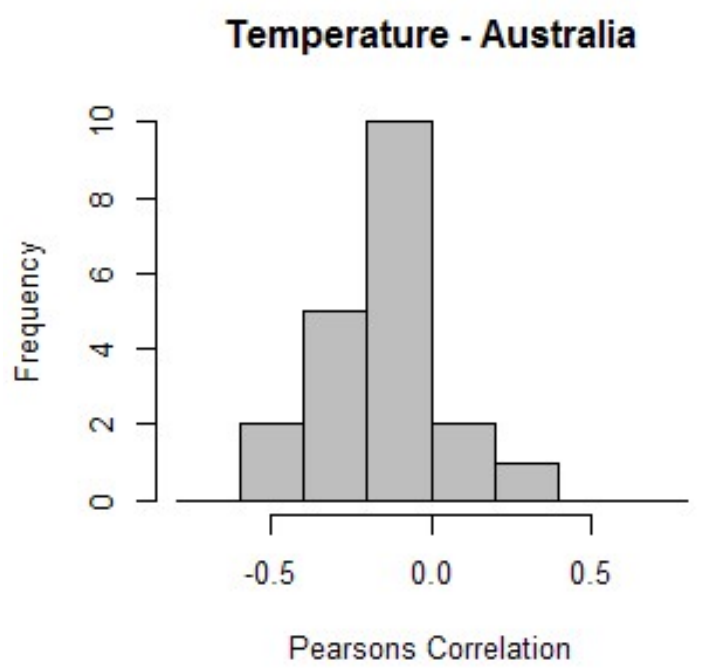

Moisture Index - Australia

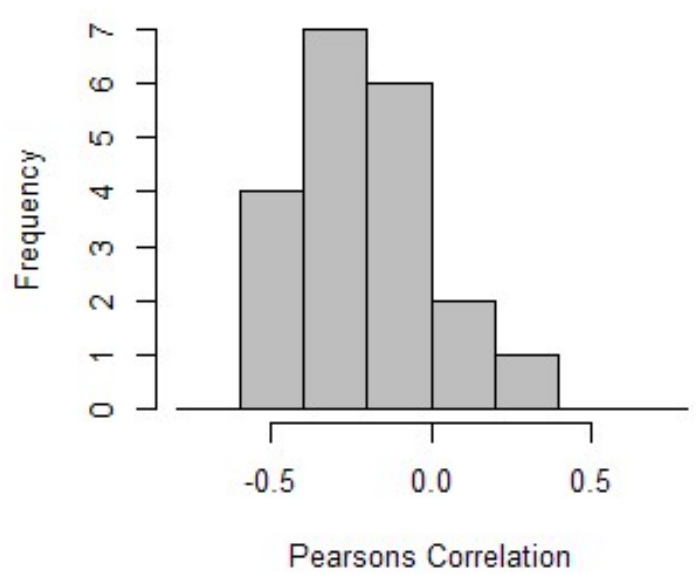

Temperature - New Zealand

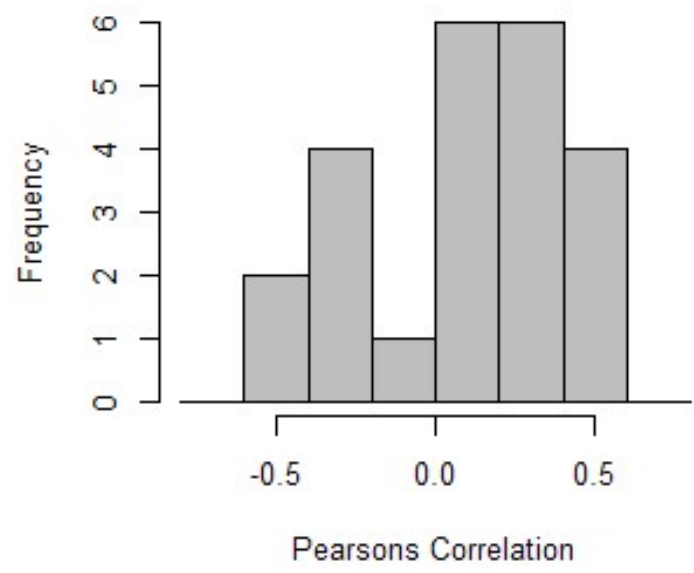

Moisture Index - New Zealand

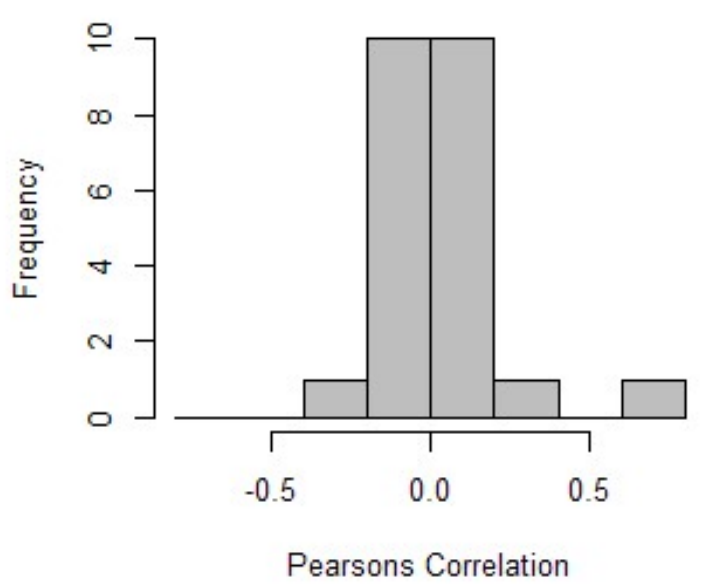

Figure S1. Frequency histograms of the Pearsons' correlation coefficients for the temperature $\left({ }^{\circ} \mathrm{C}\right)$ and moisture index of each sample location with the year it was collected, for each species in Australia and New Zealand. Positive correlation coefficients indicate that the climate (moisture; temperature) of the location of the herbarium specimen samples for a species increased through time, and vice versa. 
Figures S2 - S8. Predictions from the best fitting GLS models which included a significant term for year, for species introduced to Australia and New Zealand.

A. leptocalados Year

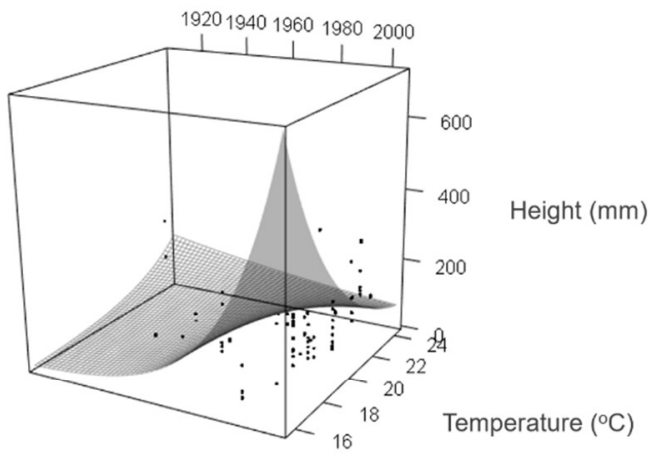

F. retusa
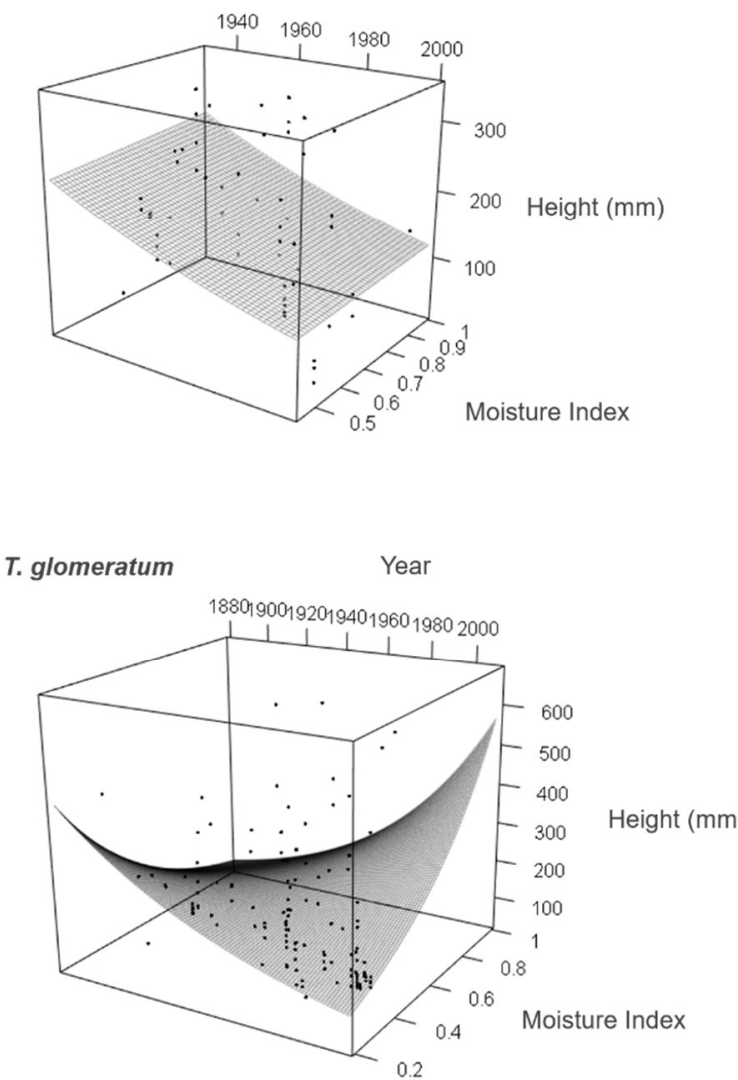

E. peplus Year
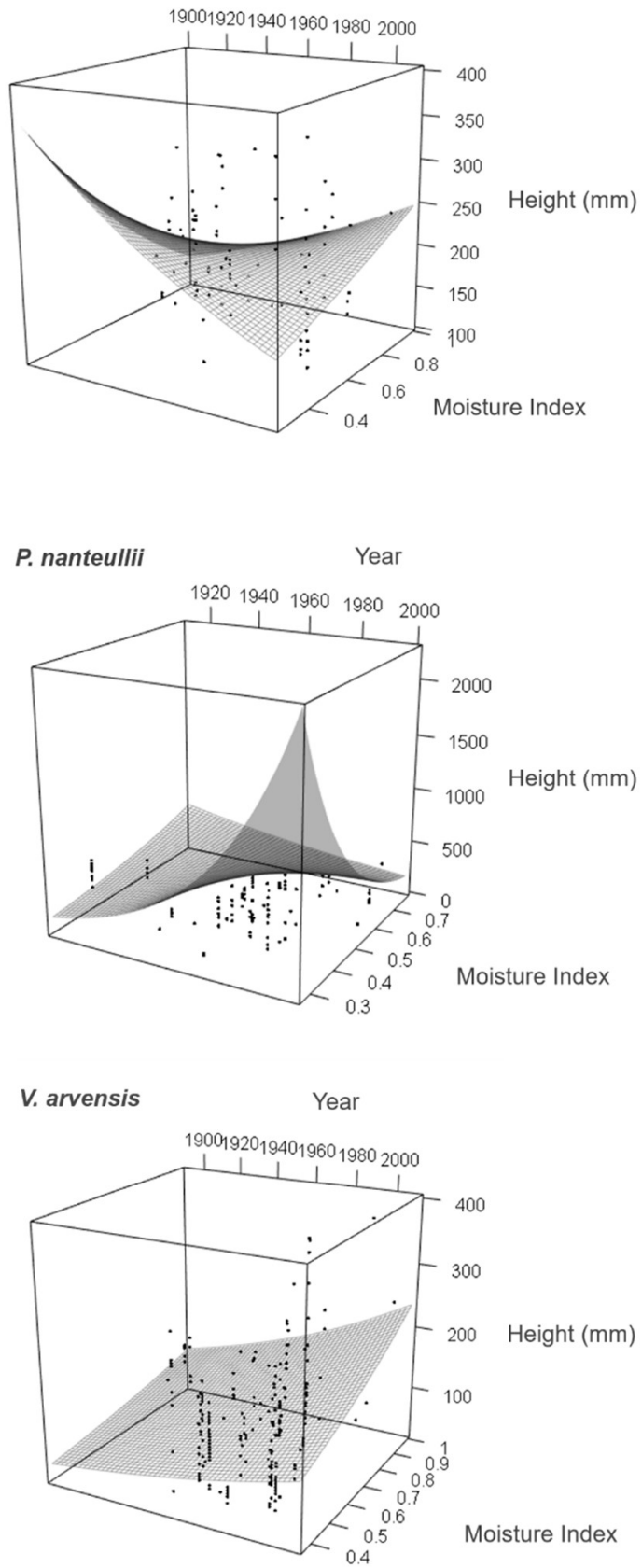

Figure S2. Species introduced to Australia in which year had a significant effect upon plant height in the best fitting model. Slopes are predicted from a GLS analysis. Arenaria leptoclados, Euphorbia peplus, Petrorhagia nanteullii and Trifolium glomeratum all showed a significant year $\times$ climate interaction. Year and climate were predicted to have a significant effect on Veronica arvensis, while just year was predicted to have a significant effect on Facelis retusa. 
Year

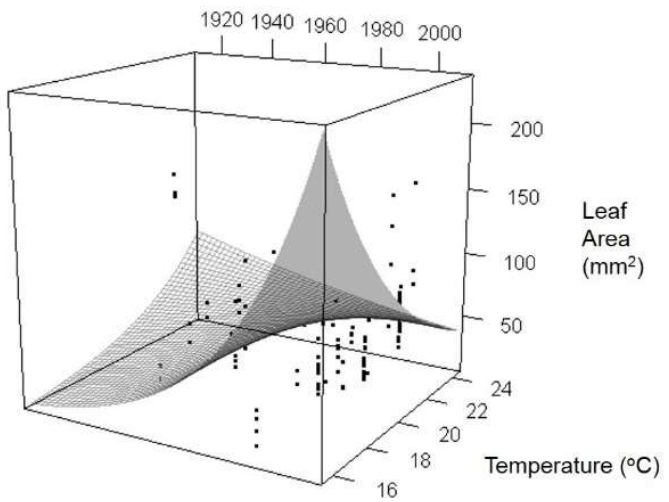

16

G. molle

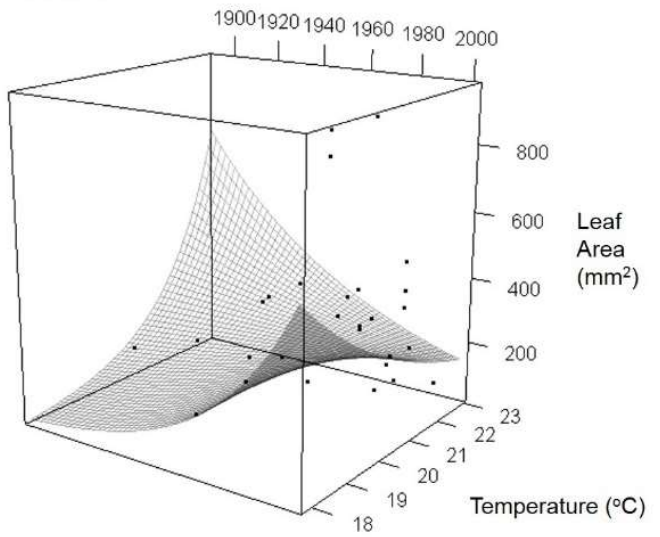

C. quadrangularis

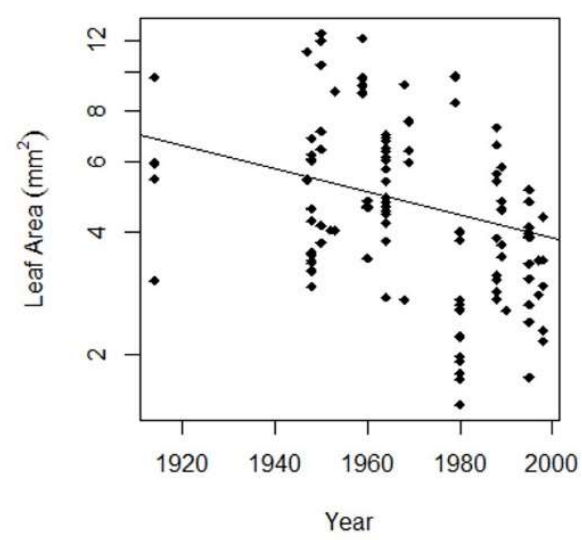

M. Iupulina

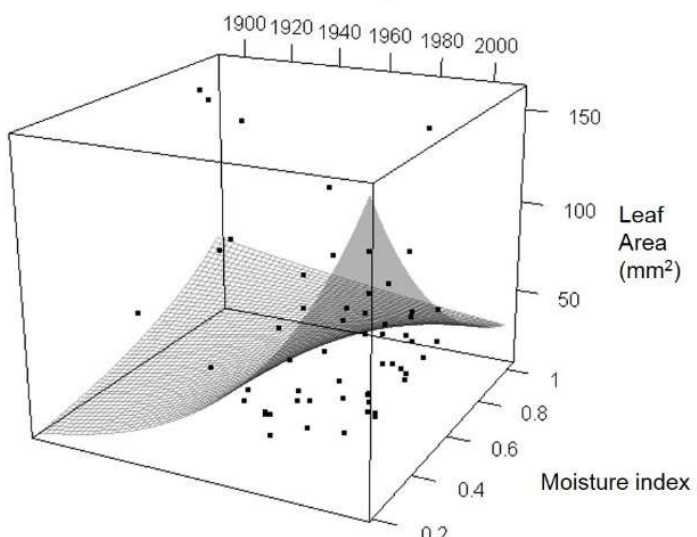

P. tetraphyllum

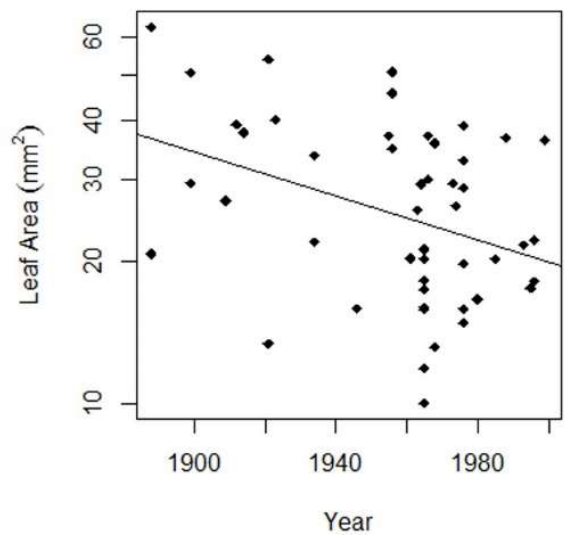

Figure S3. Species introduced to Australia, in which year showed a significant effect on leaf area in the best fitting model. Slopes are predicted from a GLS analysis. Arenaria leptoclados, Geranium molle, Medicago lupulina showed a significant year $\times$ climate interaction. Cicendia quadrangularis and Polycarpon tetraphyllum, showed a significant effect of year. 
A. populifolia

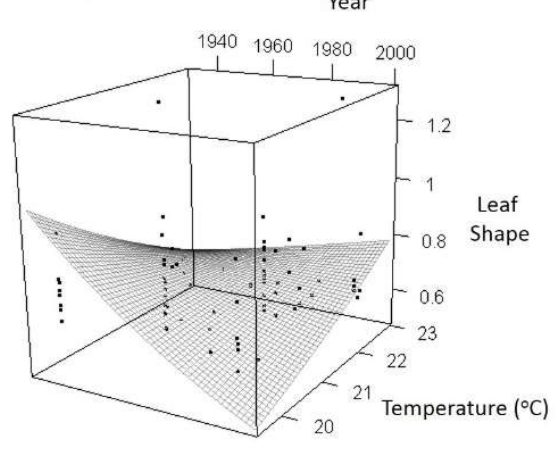

E. peplus

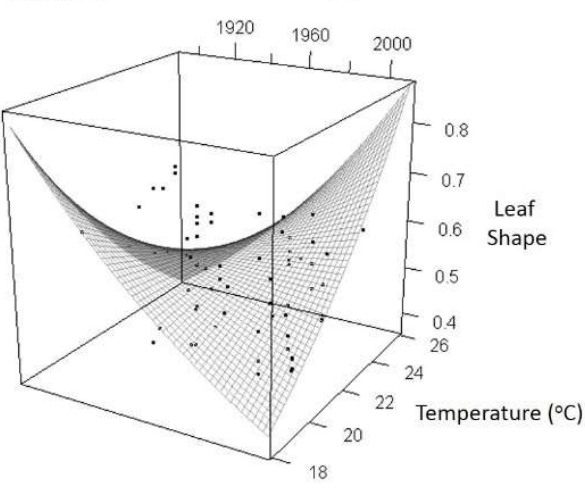

G. molle

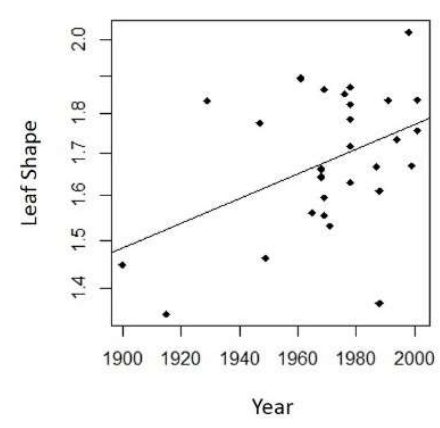

V. arvensis Year

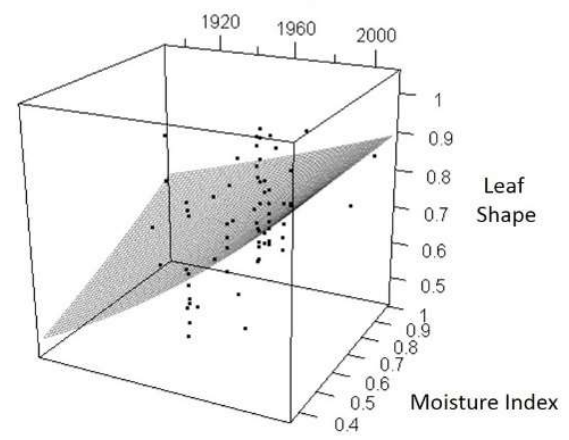

C. quadrangularis Year

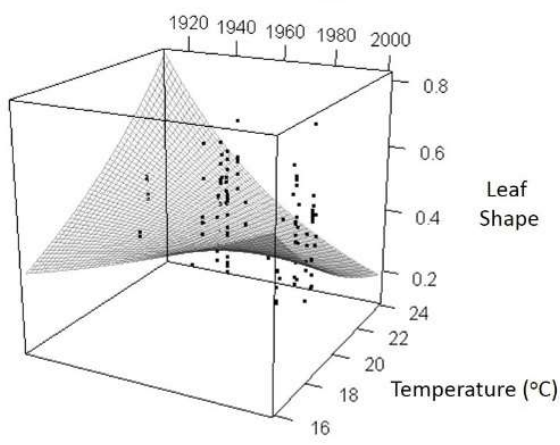

G. americana

Year

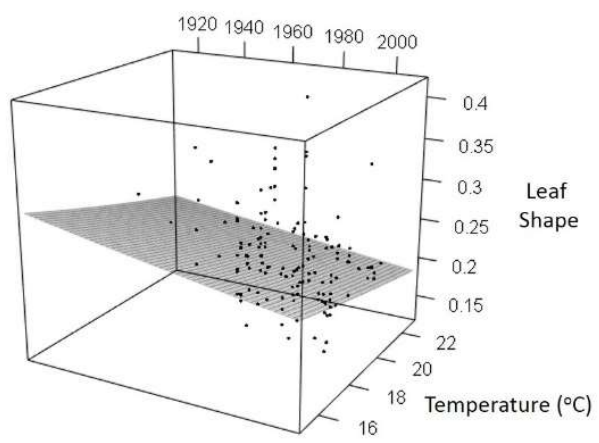

S. arvensis Year

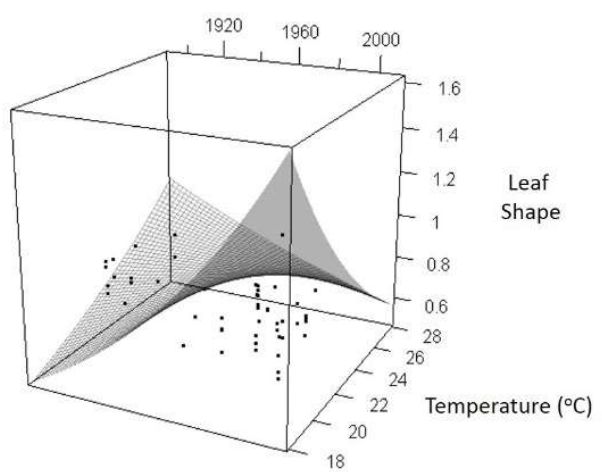

Figure S4. Species introduced to Australia, in which leaf shape showed a significant effect of year in the best fitting model. Slopes are predicted from a GLS analysis. Arctotheca populifolia, Cicendia quadrangularis, Euphorbia peplus, Stachys arvensis each showed a significant year $\times$ climate interaction. Gamochaeta americana and Veronica arvensis showed a significant effect for year and climate. Geraniume molle showed a significant effect of just year. 


\section{E. peplus}

Year

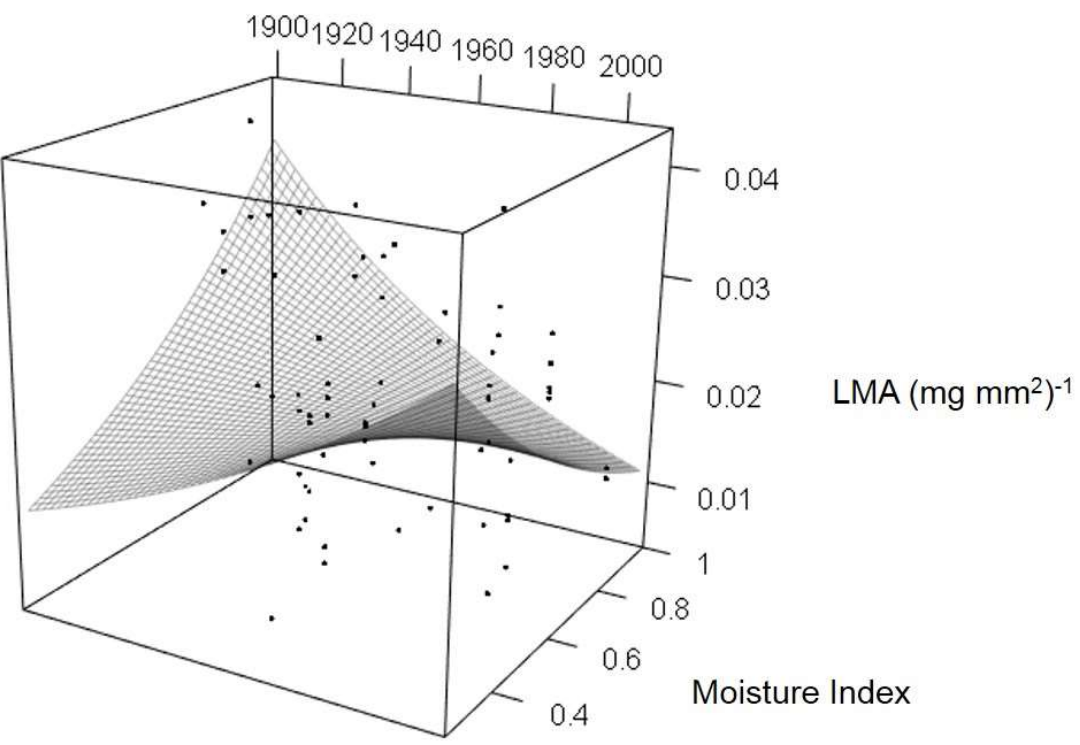

\section{T. dubium}

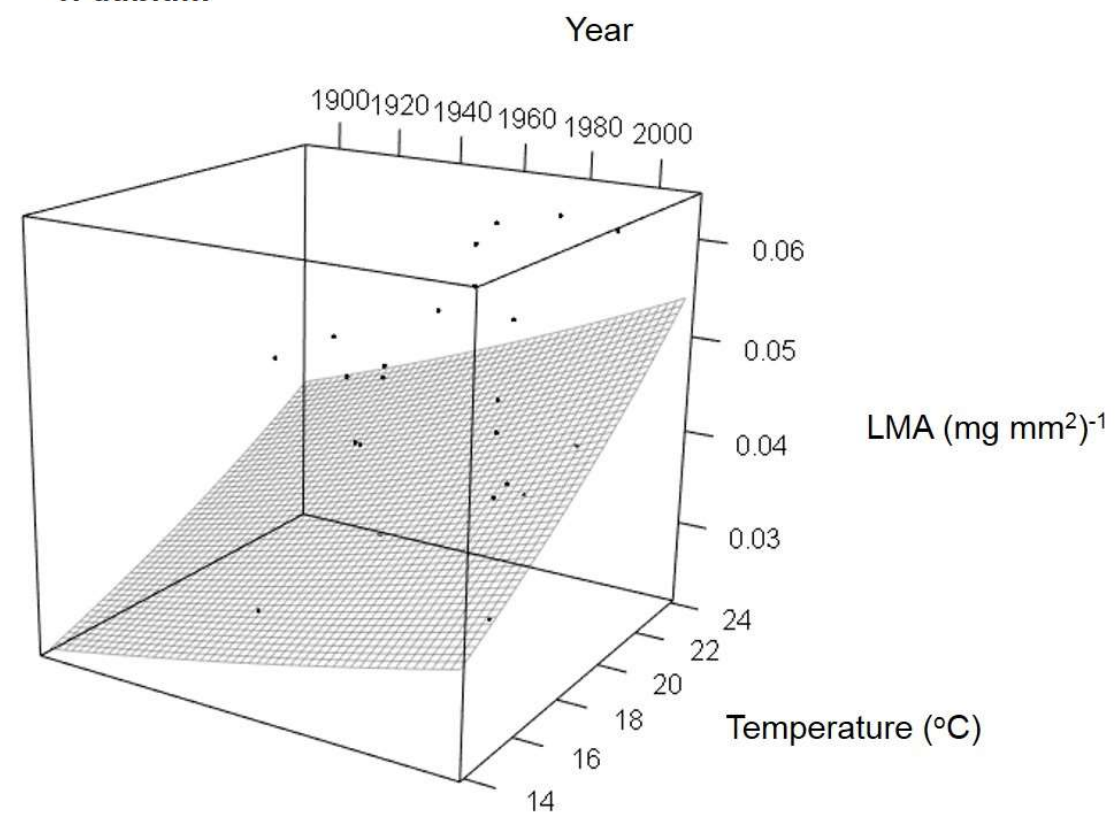

Figure S5. Models in which leaf mass per unit area (LMA) showed a significant effect of year in the best fitting model for species introduced to Australia. Euphorbia peplus showed a significant interaction of climate $\times$ year. Trifolium dubium showed a significant effect of year. 

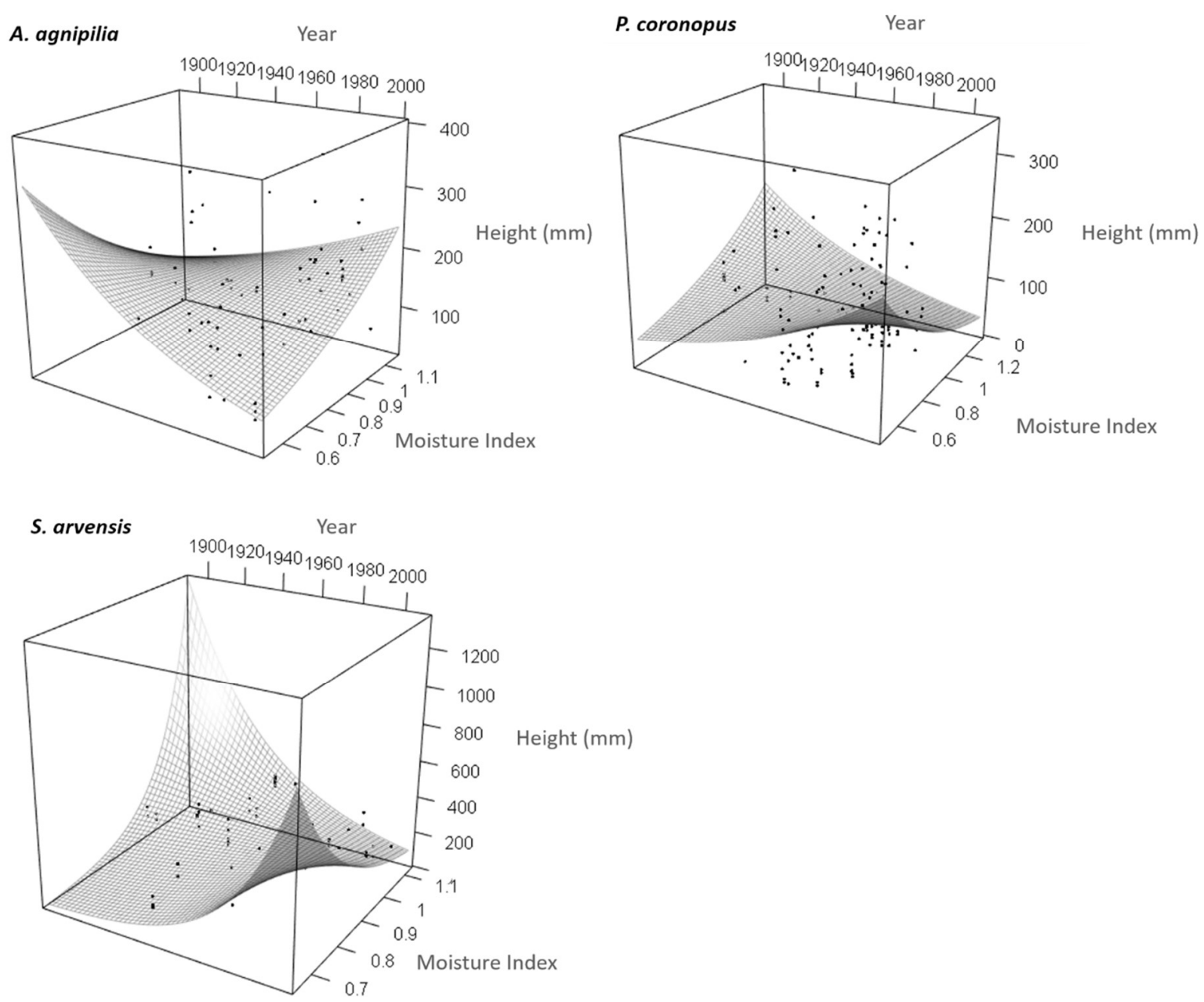

Figure S6. Models in which plant height showed a significant effect of year in the best fitting model for species introduced to New Zealand. Acaena agnipila, Plantago coronopus and Stachys arvensis all showed a significant year $\times$ climate interaction. 

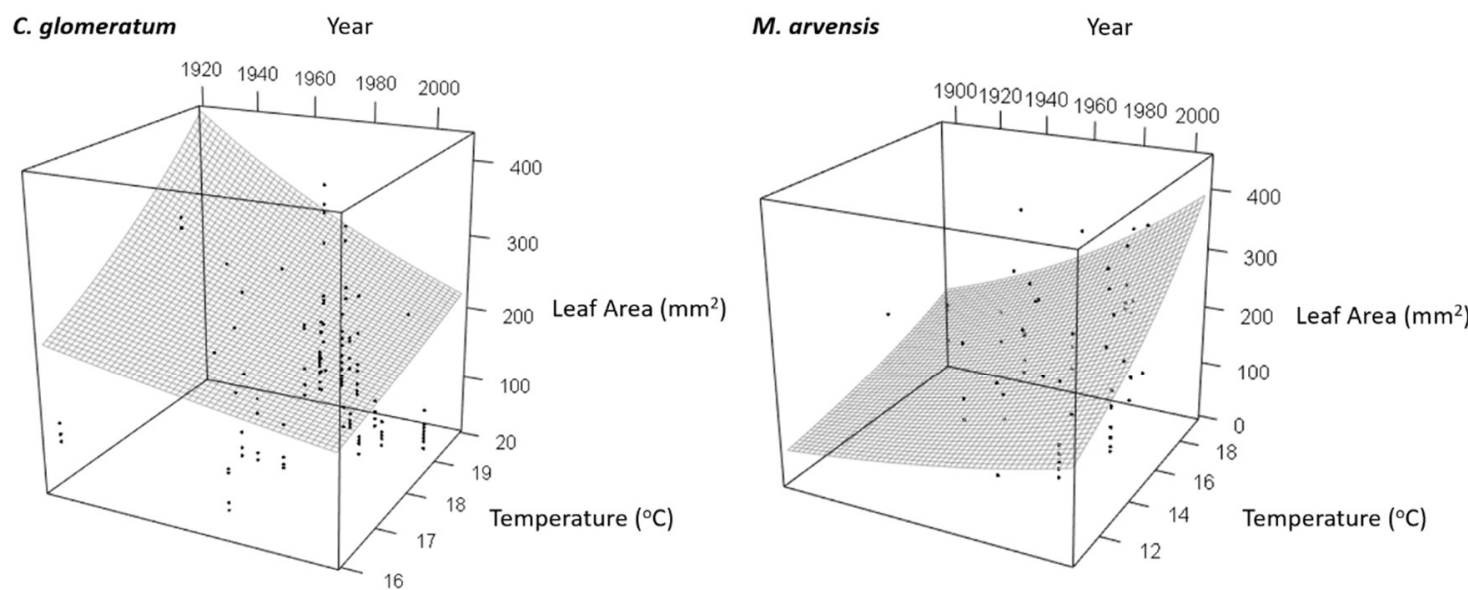

M. Iupulina
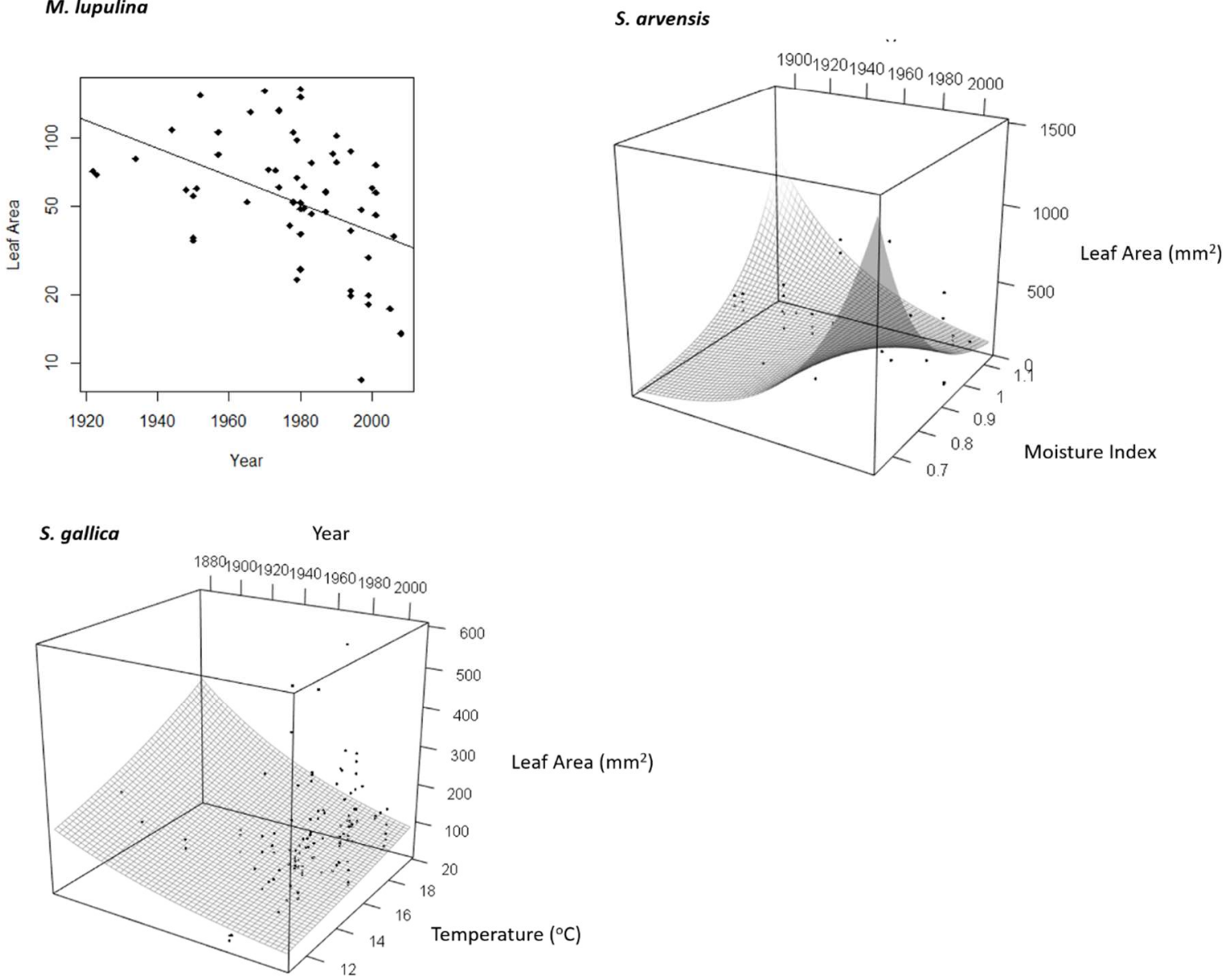

Figure S7. Models in which leaf area $\left(\mathrm{mm}^{2}\right)$ showed a significant effect of year in the best fitting model for species introduced to New Zealand. Cerastium glomeratum, Stachys arvensis and Silene gallica all showed a significant climate $\times$ year interaction. Myosotis arvensis showed a significant effect for year + climate. Medicago lupulina showed a significant effect for year. 


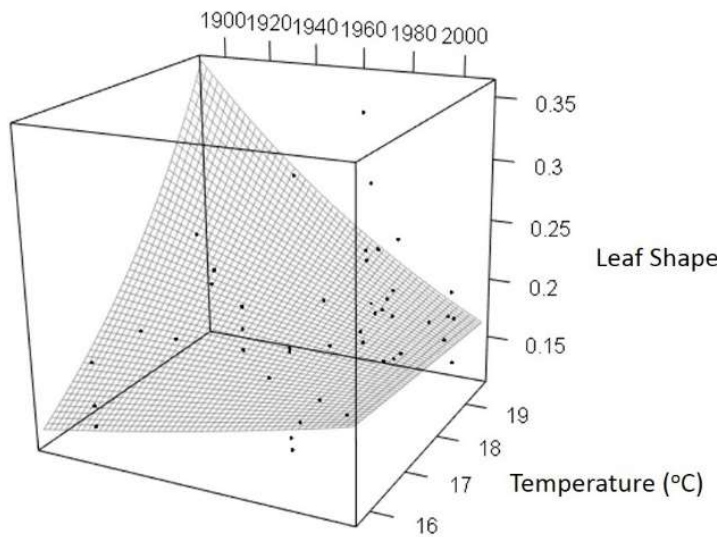

M. Iupulina

Year

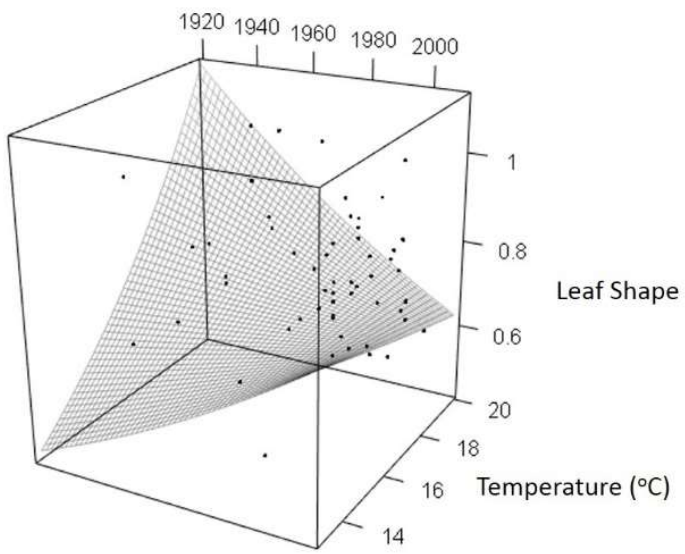

14

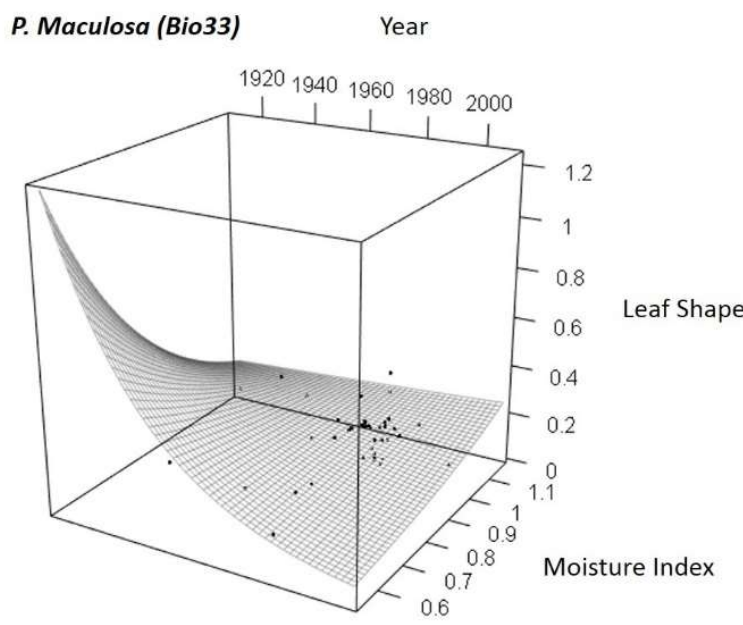

M. arvensis

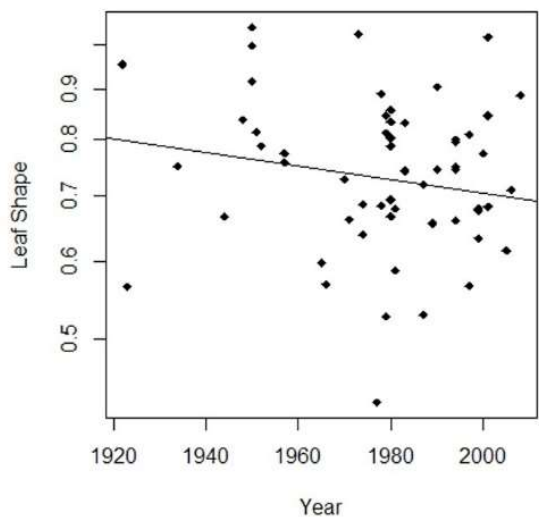

P. Maculosa (Bio10) Year

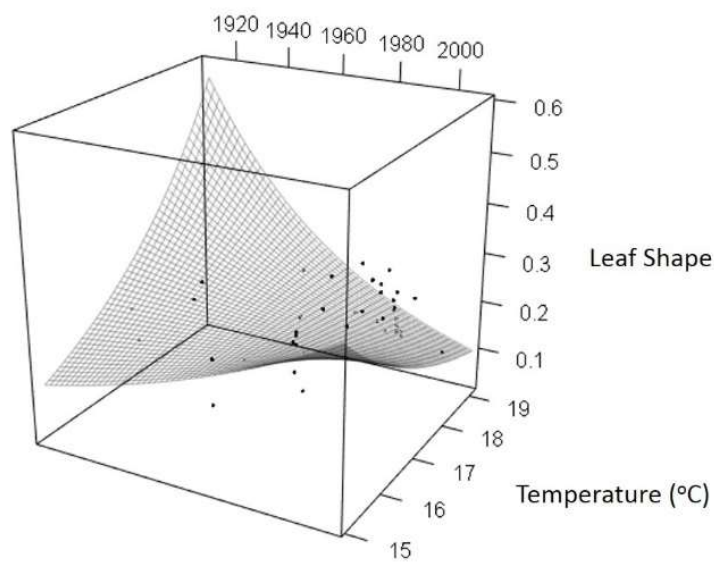

S. gallica

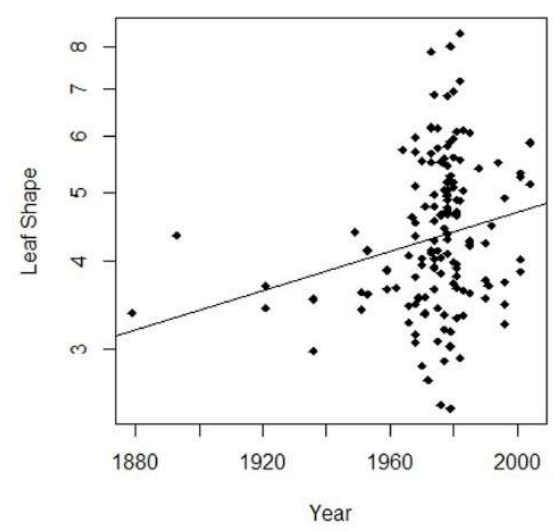

Figure S8. Models in which leaf area showed a significant effect of year in the best fitting model for species introduced to New Zealand. Lythrum hyssopifolia, Medicago lupulin, and Perscaria maculosa all showed a significant year $\times$ climate interaction. Myosotis arvensis and Silene gallica both showed a significant effect for year. 
Table S1. The loadings for each climate variable along the first two axes of the principle component analysis (PC1 and PC2) for herbarium specimens recorded in Australian and New Zealand. The two climate variables selected to be used in this study (Bio10 and Bio33) are highlighted in bold. The proportion of variation and cumulative proportion of variance accounted for along each axis is also included.

\begin{tabular}{|c|c|c|c|c|c|}
\hline \multirow{2}{*}{$\begin{array}{l}\text { CliMond } \\
\text { Variable }\end{array}$} & \multirow{2}{*}{ Variable Name } & \multicolumn{2}{|c|}{ Australia } & \multicolumn{2}{|c|}{ New Zealand } \\
\hline & & PC1 & PC2 & PC1 & PC2 \\
\hline Bio01 & Annual mean temperature $\left({ }^{\circ} \mathrm{C}\right)$ & 0.049 & -0.262 & 0.048 & 0.261 \\
\hline Bio02 & $\begin{array}{l}\text { Mean diurnal temperature range } \\
(\text { mean }(\text { period } \max -\min ))\left({ }^{\circ} \mathrm{C}\right)\end{array}$ & -0.225 & -0.017 & -0.071 & -0.165 \\
\hline Bio03 & Isothermality (Bio02 $\div$ Bio07) & 0.057 & -0.225 & 0.011 & 0.084 \\
\hline Bio04 & Temperature seasonality ( $\mathrm{C}$ of $\mathrm{V}$ ) & -0.224 & 0.076 & -0.087 & -0.210 \\
\hline Bio05 & Max temperature of warmest week $\left({ }^{\circ} \mathrm{C}\right)$ & -0.167 & -0.160 & 0.035 & 0.239 \\
\hline Bio06 & Min temperature of coldest week $\left({ }^{\circ} \mathrm{C}\right)$ & 0.137 & -0.186 & 0.063 & 0.260 \\
\hline Bio07 & Temperature annual range (Bio05-Bio06) $\left({ }^{\circ} \mathrm{C}\right)$ & -0.232 & 0.040 & -0.073 & -0.187 \\
\hline Bio08 & Mean temperature of wettest quarter $\left({ }^{\circ} \mathrm{C}\right)$ & 0.067 & -0.298 & 0.043 & 0.145 \\
\hline Bio09 & Mean temperature of driest quarter $\left({ }^{\circ} \mathrm{C}\right)$ & -0.002 & 0.051 & 0.049 & 0.253 \\
\hline Bio10 & Mean temperature of warmest quarter $\left({ }^{\circ} \mathrm{C}\right)$ & $\underline{-0.037}$ & $\underline{-0.252}$ & $\underline{0.041}$ & $\underline{0.257}$ \\
\hline Bio11 & Mean temperature of coldest quarter $\left({ }^{\circ} \mathrm{C}\right)$ & 0.103 & -0.240 & 0.059 & 0.261 \\
\hline Bio12 & Annual precipitation $(\mathrm{mm})$ & 0.235 & 0.027 & 0.231 & -0.081 \\
\hline Bio13 & Precipitation of wettest week (mm) & 0.224 & -0.039 & 0.235 & -0.048 \\
\hline Bio14 & Precipitation of driest week (mm) & 0.195 & 0.121 & 0.224 & -0.078 \\
\hline Bio15 & Precipitation seasonality (C of V) & 0.111 & -0.177 & 0.056 & 0.165 \\
\hline Bio16 & Precipitation of wettest quarter $(\mathrm{mm})$ & 0.224 & -0.026 & 0.237 & -0.044 \\
\hline Bio17 & Precipitation of driest quarter (mm) & 0.211 & 0.112 & 0.220 & -0.106 \\
\hline Bio18 & Precipitation of warmest quarter (mm) & 0.214 & -0.138 & 0.217 & -0.112 \\
\hline Bio19 & Precipitation of coldest quarter (mm) & 0.177 & 0.167 & 0.236 & -0.014 \\
\hline Bio20 & Annual mean radiation $\left(\mathrm{W} \mathrm{m}^{-2}\right)$ & -0.101 & -0.199 & 0.073 & 0.226 \\
\hline Bio21 & Highest weekly radiation $\left(\mathrm{W} \mathrm{m}^{-2}\right)$ & -0.196 & 0.017 & -0.009 & 0.145 \\
\hline Bio22 & Lowest weekly radiation $\left(\mathrm{W} \mathrm{m}^{-2}\right.$ & 0.000 & -0.277 & 0.103 & 0.227 \\
\hline Bio23 & Radiation seasonality ( $\mathrm{C}$ of $\mathrm{V})$ & -0.086 & 0.283 & -0.110 & -0.216 \\
\hline Bio24 & Radiation of wettest quarter $\left(\mathrm{W} \mathrm{m}^{-2}\right)$ & -0.034 & -0.232 & 0.048 & -0.150 \\
\hline Bio25 & Radiation of driest quarter $\left(\mathrm{W} \mathrm{m}^{-2}\right)$ & 0.003 & 0.101 & 0.035 & 0.212 \\
\hline Bio26 & Radiation of warmest quarter $\left(\mathrm{W} \mathrm{m}^{-2}\right)$ & -0.201 & 0.036 & -0.046 & 0.006 \\
\hline Bio27 & Radiation of coldest quarter $\left(\mathrm{W} \mathrm{m}^{-2}\right)$ & 0.037 & -0.278 & 0.107 & 0.228 \\
\hline Bio28 & Annual mean moisture index & 0.236 & 0.098 & 0.236 & -0.056 \\
\hline Bio29 & Highest weekly moisture index & 0.203 & 0.161 & 0.236 & -0.033 \\
\hline Bio30 & Lowest weekly moisture index & 0.234 & -0.014 & 0.224 & -0.043 \\
\hline Bio31 & Moisture index seasonality ( $\mathrm{C}$ of $\mathrm{V}$ ) & -0.122 & 0.190 & -0.148 & 0.033 \\
\hline Bio32 & Mean moisture index of wettest quarter & 0.196 & 0.176 & 0.237 & -0.031 \\
\hline Bio33 & Mean moisture index of driest quarter & $\underline{0.238}$ & $\underline{-0.014}$ & $\underline{0.224}$ & $\underline{-0.059}$ \\
\hline Bio34 & Mean moisture index of warmest quarter & 0.238 & -0.077 & 0.226 & -0.053 \\
\hline Bio35 & Mean moisture index of coldest quarter & 0.180 & 0.208 & 0.236 & -0.016 \\
\hline \multicolumn{2}{|c|}{ Proportion of Variance } & 0.457 & 0.270 & 0.417 & 0.320 \\
\hline \multicolumn{2}{|c|}{ Cumulative Proportion of Variance } & & $\underline{0.727}$ & & $\underline{0.737}$ \\
\hline
\end{tabular}


Table S2. Results for the best fitting GLS models to explain variation in plant height for each species introduced to Australia. Delta AIC is calculated in reference to the null model. Negative Delta AIC's indicate that the null model was better than the best fitting model Change with GLS?' refers to if the best fitting model in the updated GLS approach (including climate and coordinate data), gave a different significant effect for the year (or year $\mathrm{x}$ climate term).

\begin{tabular}{|c|c|c|c|c|c|c|c|c|c|}
\hline Species & $\begin{array}{c}\text { Year } \\
\text { significant in } \\
\text { original study? }\end{array}$ & $\begin{array}{c}\text { Year } \\
\text { significant in } \\
\text { new GLM? }\end{array}$ & $\begin{array}{l}\text { Terms included in } \\
\text { best model }\end{array}$ & $B$ & $p$ & $n$ & $\begin{array}{l}\text { Delta } \\
\text { AIC }\end{array}$ & $\begin{array}{l}\text { Change } \\
\text { with } \\
\text { GLS? }\end{array}$ & $\begin{array}{l}\text { Explanation of } \\
\text { change with GLS }\end{array}$ \\
\hline $\begin{array}{l}\text { Arenaria } \\
\text { leptoclados }\end{array}$ & No & No & $\begin{array}{l}\text { Year } \\
\text { Temperature } \\
\text { Moisture } \\
\text { Year } \times \text { Temperature } \\
\text { Year } \times \text { Moisture }\end{array}$ & $\begin{array}{r}0.077 \\
5.267 \\
91.267 \\
-0.003 \\
-0.046 \\
\end{array}$ & $\begin{array}{r}\frac{<0.001}{<0.001} \\
\underline{0.041} \\
\underline{\leq 0.001} \\
\underline{0.040} \\
\end{array}$ & 115 & 12.63 & Yes & $\begin{array}{l}\text { Adding climate data } \\
\text { reveals a significant } \\
\text { effect of year }\end{array}$ \\
\hline $\begin{array}{l}\text { Arctotheca } \\
\text { populifolia }\end{array}$ & Yes & No & Year & 0.002 & 0.109 & 36 & -5.26 & No & \\
\hline $\begin{array}{l}\text { Cicendia } \\
\text { quadrangulis }\end{array}$ & No & No & Year & 0.0011 & 0.217 & 122 & 1.37 & No & \\
\hline $\begin{array}{l}\text { Euphorbia } \\
\text { peplus }\end{array}$ & No & No & $\begin{array}{l}\text { Year } \\
\text { Moisture } \\
\text { Year } \times \text { Moisture }\end{array}$ & $\begin{array}{r}-0.005 \\
-14.987 \\
0.008 \\
\end{array}$ & $\begin{array}{l}\frac{0.03}{0.018} \\
\underline{0.018}\end{array}$ & 91 & 1.79 & Yes & \\
\hline $\begin{array}{l}\text { Facelis } \\
\text { retusa }\end{array}$ & Yes & Yes & Year & -0.005 & $\underline{0.039}$ & 58 & 2.32 & No & \\
\hline $\begin{array}{l}\text { Gamochaeta } \\
\text { americana }\end{array}$ & No & No & $\begin{array}{l}\text { Year } \\
\text { Moisture }\end{array}$ & -0.0001 & 0.954 & 139 & -1.43 & No & \\
\hline $\begin{array}{l}\text { Geraniume } \\
\text { molle }\end{array}$ & No & No & Year & -0.001 & 0.306 & 108 & -0.95 & No & \\
\hline $\begin{array}{l}\text { Medicago } \\
\text { polymorpha }\end{array}$ & No & No & $\begin{array}{l}\text { Year } \\
\text { Moisture }\end{array}$ & $\begin{array}{l}0.001 \\
0.380 \\
\end{array}$ & $\begin{array}{l}0.268 \\
0.097 \\
\end{array}$ & 62 & 0.52 & No & \\
\hline $\begin{array}{l}\text { Medicago } \\
\text { lupulina }\end{array}$ & No & No & Year & 0.001 & 0.45 & 54 & -1.4 & No & \\
\hline
\end{tabular}




\begin{tabular}{|c|c|c|c|c|c|c|c|c|c|}
\hline $\begin{array}{l}\text { Petrorhagia } \\
\text { nanteulli }\end{array}$ & Yes & No & $\begin{array}{l}\text { Year } \\
\text { Moisture } \\
\text { Year } \times \text { Moisture }\end{array}$ & $\begin{array}{r}0.015 \\
71.029 \\
-0.035 \\
\end{array}$ & $\begin{array}{l}\underline{0.008} \\
0.007 \\
\underline{0.007} \\
\end{array}$ & 137 & 1.18 & Yes & $\begin{array}{l}\text { Adding climate data } \\
\text { reveals a significant } \\
\text { effect of year }\end{array}$ \\
\hline $\begin{array}{l}\text { Polycarpon } \\
\text { tetraphyllum }\end{array}$ & $\begin{array}{l}\text { Yes (Arid- } \\
\text { Region) }\end{array}$ & No & Year & -0.001 & 0.302 & 103 & -0.93 & No & \\
\hline Silene gallica & Yes (Semi-arid) & $\begin{array}{l}\text { Yes (Semi- } \\
\text { arid) }\end{array}$ & Year & -0.0003 & 0.772 & 74 & -1.91 & No & \\
\hline $\begin{array}{l}\text { Stachys } \\
\text { arvensis }\end{array}$ & No & No & Year & 0.0002 & 0.791 & 93 & -1.94 & No & \\
\hline $\begin{array}{l}\text { Trifolium } \\
\text { dubium }\end{array}$ & No & No & Year & 0.0006 & $\underline{0.002}$ & 102 & -1.9 & Yes & $\begin{array}{l}\text { Adding coordinate data } \\
\text { reveals a significant } \\
\text { effect of year }\end{array}$ \\
\hline $\begin{array}{l}\text { Trifolium } \\
\text { glomeratum }\end{array}$ & Yes (arid) & Yes & $\begin{array}{l}\text { Year } \\
\text { Moisture } \\
\text { Year } \times \text { Moisture }\end{array}$ & $\begin{array}{r}-0.010 \\
-29.173 \\
0.015\end{array}$ & $\begin{array}{r}<0.001 \\
\underline{0.008} \\
\underline{0.008}\end{array}$ & 129 & 69.48 & No & \\
\hline $\begin{array}{l}\text { Veronica } \\
\text { arvensis }\end{array}$ & Yes & Yes & $\begin{array}{l}\text { Year } \\
\text { Moisture } \\
\text { Temperature }\end{array}$ & $\begin{array}{l}0.003 \\
0.012 \\
0.444\end{array}$ & $\begin{array}{l}\underline{0.007} \\
0.209 \\
\underline{0.004} \\
\end{array}$ & 67 & 5.8 & No & \\
\hline
\end{tabular}


Table S3. Results for the best fitting models to explain variation in leaf area for each species introduced to Australia. Delta AIC is calculated in reference to the null model. Negative Delta AIC's indicate that the null model was better than the best fitting model. Change with GLS?' refers to if the best fitting model in the updated GLS approach (including climate and coordinate data), gave a different significant effect for the year (or year $\mathrm{x}$ climate term).

\begin{tabular}{|c|c|c|c|c|c|c|c|c|c|}
\hline Species & $\begin{array}{l}\text { Year } \\
\text { significant in } \\
\text { original } \\
\text { study? }\end{array}$ & $\begin{array}{c}\text { Year } \\
\text { significant in } \\
\text { new GLM? }\end{array}$ & $\begin{array}{l}\text { Terms included in } \\
\text { best model }\end{array}$ & $B$ & $p$ & $n$ & Delta AIC & $\begin{array}{l}\text { Change } \\
\text { with } \\
\text { GLS? }\end{array}$ & $\begin{array}{l}\text { Explanation of } \\
\text { change with GLS }\end{array}$ \\
\hline $\begin{array}{l}\text { Arenaria } \\
\text { leptoclados }\end{array}$ & No & No & $\begin{array}{l}\text { Year } \\
\text { Temperature } \\
\text { Year } \times \text { Temperature }\end{array}$ & $\begin{array}{l}0.0491 \\
3.9529 \\
-0.002\end{array}$ & $\begin{array}{l}\frac{0.022}{0.021} \\
\underline{0.022}\end{array}$ & 122 & 12.62 & Yes & $\begin{array}{l}\text { Adding climate } \\
\text { data reveals a } \\
\text { significant effect of } \\
\text { year }\end{array}$ \\
\hline $\begin{array}{l}\text { Arctotheca } \\
\text { populifolia }\end{array}$ & No & No & $\begin{array}{l}\text { Year } \\
\text { Temperature }\end{array}$ & $\begin{array}{r}0.0001 \\
-0.1138\end{array}$ & $\begin{array}{r}0.802 \\
\leq 0.001 \\
\end{array}$ & 52 & 2.41 & No & \\
\hline $\begin{array}{l}\text { Cicendia } \\
\text { quadrangulis }\end{array}$ & Yes (semi-arid) & Yes & Year & -0.003 & $\underline{0.033}$ & 111 & 1.88 & No & \\
\hline $\begin{array}{l}\text { Euphorbia } \\
\text { peplus }\end{array}$ & No & No & Year & 0.0004 & 0.723 & 69 & 1.87 & No & \\
\hline $\begin{array}{l}\text { Gamochaeta } \\
\text { americana }\end{array}$ & No & No & Year & -0.0013 & 0.449 & 141 & -32.34 & No & \\
\hline $\begin{array}{l}\text { Geraniume } \\
\text { molle }\end{array}$ & No & No & $\begin{array}{l}\text { Year } \\
\text { Temperature } \\
\text { Year } \times \text { Temperature }\end{array}$ & $\begin{array}{l}0.0788 \\
7.175 \\
-0.0037\end{array}$ & $\begin{array}{l}\frac{0.039}{0.048} \\
\underline{0.048}\end{array}$ & 28 & 2.66 & Yes & $\begin{array}{l}\text { Adding climate } \\
\text { data reveals a } \\
\text { significant effect of } \\
\text { year }\end{array}$ \\
\hline $\begin{array}{l}\text { Medicago } \\
\text { polymorpha }\end{array}$ & No & No & $\begin{array}{l}\text { Year } \\
\text { Temperature }\end{array}$ & $\begin{array}{r}-0.0001 \\
0.0413\end{array}$ & $\begin{array}{l}0.414 \\
\mathbf{0 . 0 1 4} \\
\end{array}$ & 120 & 3.01 & No & \\
\hline $\begin{array}{l}\text { Medicago } \\
\text { lupulina }\end{array}$ & No & No & $\begin{array}{l}\text { Year } \\
\text { Moisture } \\
\text { Year } \times \text { Moisture }\end{array}$ & $\begin{array}{l}0.0093 \\
29.798 \\
-0.0145\end{array}$ & $\begin{array}{l}\frac{0.046}{0.021} \\
\underline{0.023}\end{array}$ & 57 & 3.28 & Yes & $\begin{array}{l}\text { Adding climate } \\
\text { data reveals a } \\
\text { significant effect of } \\
\text { year }\end{array}$ \\
\hline Melilotus indicus & No & No & Year & 0.0027 & 0.336 & 53 & -1.1 & No & \\
\hline
\end{tabular}




\begin{tabular}{|c|c|c|c|c|c|c|c|c|c|}
\hline $\begin{array}{l}\text { Polycarpon } \\
\text { tetraphyllum }\end{array}$ & No & Yes & Year & -0.0021 & $\underline{0.024}$ & 49 & 6.96 & No & \\
\hline Silene gallica & No & No & Year & -3.81 & -0.0014 & 78 & 0.121 & No & \\
\hline $\begin{array}{l}\text { Stachys } \\
\text { arvensis }\end{array}$ & No & No & Year & -0.0016 & 0.269 & 93 & -0.74 & No & \\
\hline Trifolium dubium & No & Yes & $\begin{array}{l}\text { Year } \\
\text { Temperature }\end{array}$ & $\begin{array}{l}0.0006 \\
0.048\end{array}$ & $\begin{array}{r}0.5693 \\
< \\
\end{array}$ & 119 & 7.89 & Yes & $\begin{array}{l}\text { Adding climate } \\
\text { and coordinate } \\
\text { data removes a } \\
\text { significant effect of } \\
\text { year }\end{array}$ \\
\hline $\begin{array}{l}\text { Trifolium } \\
\text { glomeratum }\end{array}$ & No & No & $\begin{array}{l}\text { Year } \\
\text { Temperature } \\
\text { Moisture } \\
\text { Year } \times \text { Moisture }\end{array}$ & $\begin{array}{l}0.0094 \\
0.9232 \\
-0.213 \\
-0.0005\end{array}$ & $\begin{array}{l}0.485 \\
0.448 \\
0.153 \\
0.461\end{array}$ & 76 & 76 & No & \\
\hline $\begin{array}{l}\text { Veronica } \\
\text { arvensis }\end{array}$ & Yes & Yes & $\begin{array}{l}\text { Year } \\
\text { Temperature } \\
\text { Moisture } \\
\text { Year } \times \text { Temperature }\end{array}$ & $\begin{array}{r}-0.0016 \\
0.0295 \\
-16.8018 \\
0.4442\end{array}$ & $\begin{array}{l}0.702 \\
\underline{\mathbf{0 . 0 2 1}} \\
0.123 \\
0.12\end{array}$ & 67 & 10.92 & Yes & $\begin{array}{l}\text { Adding climate } \\
\text { and coordinate } \\
\text { data removes a } \\
\text { significant effect of } \\
\text { year }\end{array}$ \\
\hline
\end{tabular}


Table S4. Results for the best fitting models to explain variation in leaf shape for each species introduced to Australia. Delta AIC is calculated in reference to the null model. Negative Delta AIC's indicate that the null model was better than the best fitting model. Change with GLS?' refers to if the best fitting model in the updated GLS approach (including climate and coordinate data), gave a different significant effect for the year (or year $\mathrm{x}$ climate term).

\begin{tabular}{|c|c|c|c|c|c|c|c|c|c|}
\hline Species & $\begin{array}{l}\text { Year } \\
\text { significant in } \\
\text { original } \\
\text { study? }\end{array}$ & $\begin{array}{c}\text { Year } \\
\text { significant } \\
\text { in new } \\
\text { GLM? }\end{array}$ & $\begin{array}{l}\text { Terms included in best } \\
\text { GLS model }\end{array}$ & $n$ & $B$ & $p$ & $\begin{array}{l}\text { Delta } \\
\text { AIC }\end{array}$ & $\begin{array}{l}\text { Change } \\
\text { with } \\
\text { GLS? }\end{array}$ & $\begin{array}{l}\text { Explanation of } \\
\text { change with GLS }\end{array}$ \\
\hline $\begin{array}{l}\text { Arenaria } \\
\text { leptoclados }\end{array}$ & No & No & Year & 122 & 0.00004 & 0.934 & -1.99 & No & \\
\hline $\begin{array}{l}\text { Arctotheca } \\
\text { populifolia }\end{array}$ & No & $\begin{array}{c}\text { No } \\
(p=0.064)\end{array}$ & $\begin{array}{l}\text { Year } \\
\text { Temperature } \\
\text { Year } \times \text { Temperature }\end{array}$ & 52 & $\begin{array}{l}-0.026 \\
-2.476 \\
0.001\end{array}$ & $\begin{array}{l}0.05 \\
\underline{0.042} \\
\underline{\mathbf{0 . 0 4 3}} \\
\end{array}$ & 0.229 & Yes & $\begin{array}{l}\text { Adding climate } \\
\text { data reveals a } \\
\text { significant effect of } \\
\text { year }\end{array}$ \\
\hline $\begin{array}{l}\text { Cicendia } \\
\text { quadrangulis }\end{array}$ & No & No & $\begin{array}{l}\text { Year } \\
\text { Temperature } \\
\text { Moisture } \\
\text { Year } \times \text { Temperature } \\
\text { Year } \times \text { Moisture } \\
\text { Temperature } \times \text { Moisture }\end{array}$ & 111 & $\begin{array}{r}0.0343 \\
2.4305 \\
53.2269 \\
-0.0012 \\
-0.0257 \\
-0.1913 \\
\end{array}$ & $\begin{array}{l}\frac{0.036}{0.027} \\
\frac{0.061}{0.031} \\
\frac{0.074}{0.014} \\
\end{array}$ & 1.44 & Yes & $\begin{array}{l}\text { Adding climate } \\
\text { data reveals a } \\
\text { significant effect of } \\
\text { year }\end{array}$ \\
\hline $\begin{array}{l}\text { Euphorbia } \\
\text { peplus }\end{array}$ & No & No & $\begin{array}{l}\text { Year } \\
\text { Temperature } \\
\text { Year } \times \text { Temperature }\end{array}$ & 69 & $\begin{array}{r}-0.0167 \\
-1.4652 \\
0.0007\end{array}$ & $\begin{array}{l}\frac{0.016}{0.024} \\
\underline{0.022}\end{array}$ & 3.3 & Yes & $\begin{array}{l}\text { Adding climate } \\
\text { data reveals a } \\
\text { significant effect of } \\
\text { year }\end{array}$ \\
\hline $\begin{array}{l}\text { Gamochaeta } \\
\text { americana }\end{array}$ & Yes & No & $\begin{array}{l}\text { Year } \\
\text { Temperature }\end{array}$ & 141 & $\begin{array}{l}-0.0013 \\
-0.0117\end{array}$ & $\underline{0.015}$ & 4.44 & Yes & $\begin{array}{l}\text { Adding climate } \\
\text { data reveals a } \\
\text { significant effect of } \\
\text { year }\end{array}$ \\
\hline $\begin{array}{l}\text { Geraniume } \\
\text { molle }\end{array}$ & Yes & Yes & Year & 28 & 0.0008 & $\underline{0.021}$ & 3.80 & No & \\
\hline $\begin{array}{l}\text { Medicago } \\
\text { polymorpha }\end{array}$ & No & No & $\begin{array}{l}\text { Year } \\
\text { Temperature } \\
\text { Moisture }\end{array}$ & 120 & $\begin{array}{l}-0.0001 \\
-0.0122 \\
-0.2057\end{array}$ & $\begin{array}{r}0.532 \\
0.001 \\
\leq 0.001 \\
\end{array}$ & 10.29 & No & \\
\hline
\end{tabular}




\begin{tabular}{|c|c|c|c|c|c|c|c|c|c|}
\hline $\begin{array}{l}\text { Medicago } \\
\text { Iupulina }\end{array}$ & No & No & $\begin{array}{l}\text { Year } \\
\text { Temperature } \\
\text { Moisture }\end{array}$ & 70 & $\begin{array}{c}-0.0004 \\
-0.0104 \\
-0.098\end{array}$ & $\begin{array}{l}0.287 \\
\underline{0.049} \\
0.107\end{array}$ & 0.07 & & \\
\hline $\begin{array}{l}\text { Melilotus } \\
\text { indicus }\end{array}$ & No & No & Year & 36 & -0.0005 & 0.241 & -0.24 & No & \\
\hline $\begin{array}{l}\text { Polycarpon } \\
\text { tetraphyllum }\end{array}$ & No & No & $\begin{array}{l}\text { Year } \\
\text { Temperature }\end{array}$ & 103 & $\begin{array}{r}-0.0005 \\
0.0115\end{array}$ & $\begin{array}{l}0.206 \\
\underline{\mathbf{0 . 0 0 8}} \\
\end{array}$ & 3.80 & No & \\
\hline Silene gallica & No & No & $\begin{array}{l}\text { Year } \\
\text { Moisture }\end{array}$ & 78 & $\begin{array}{l}-0.0003 \\
-0.1905\end{array}$ & $\begin{array}{l}0.329 \\
\mathbf{0 . 0 0 2} \\
\end{array}$ & 18.13 & No & \\
\hline $\begin{array}{l}\text { Stachys } \\
\text { arvensis }\end{array}$ & No & No & $\begin{array}{l}\text { Year } \\
\text { Temperature } \\
\text { Moisture } \\
\text { Year } \times \text { Temperature } \\
\text { Year } \times \text { Moisture } \\
\text { Temperature } \times \text { Moisture }\end{array}$ & 55 & $\begin{array}{r}0.0058 \\
0.6706 \\
3.8285 \\
-0.0003 \\
0.0023 \\
-0.0332 \\
\end{array}$ & $\begin{array}{l}0.483 \\
0.33 \\
0.353 \\
0.339 \\
0.258 \\
0.149 \\
\end{array}$ & 8.51 & No & \\
\hline $\begin{array}{l}\text { Trifolium } \\
\text { dubium }\end{array}$ & No & No & $\begin{array}{l}\text { Year } \\
\text { Temperature } \\
\text { Moisture } \\
\text { Year } \times \text { Temperature } \\
\text { Year } \times \text { Moisture } \\
\text { Temperature } \times \text { Moisture }\end{array}$ & 117 & $\begin{array}{r}0.0006 \\
-0.0446 \\
-0.8587 \\
<0.0001 \\
-0.0001 \\
-0.0611\end{array}$ & $\begin{array}{l}0.849 \\
0.877 \\
0.818 \\
0.965 \\
0.945 \\
\underline{\mathbf{0 . 0 2 5}} \\
\end{array}$ & 3.05 & No & \\
\hline $\begin{array}{l}\text { Trifolium } \\
\text { glomeratum }\end{array}$ & Yes & Yes & Year & 76 & -0.0004 & 0.182 & -0.35 & No & $\begin{array}{l}\text { Adding coordinate } \\
\text { data makes year } \\
\text { no longer } \\
\text { significant }\end{array}$ \\
\hline $\begin{array}{l}\text { Veronica } \\
\text { arvensis }\end{array}$ & No & Yes & $\begin{array}{l}\text { Year } \\
\text { Temperature } \\
\text { Moisture } \\
\text { Year } \times \text { Moisture }\end{array}$ & 67 & $\begin{array}{r}0.0032 \\
0.0084 \\
4.5411 \\
-0.0023\end{array}$ & $\begin{array}{l}\frac{0.003}{\mathbf{0 . 0 1 1}} \\
0.099 \\
0.106\end{array}$ & 8.7 & Yes & $\begin{array}{l}\text { Adding coordinate } \\
\text { data makes year } \\
\text { no longer } \\
\text { significant }\end{array}$ \\
\hline
\end{tabular}


Table S5. Results for the best fitting models to explain variation in leaf mass per area for each species introduced to Australia. Delta AIC is calculated in reference to the null model. Negative Delta AIC's indicate that the null model was better than the best fitting model. 'Change with GLS?' refers to if the best fitting model in the updated GLS approach (including climate and coordinate data), gave a different significant effect for the year (or year $\mathrm{x}$ climate term).

\begin{tabular}{|c|c|c|c|c|c|c|c|c|c|}
\hline Species & $\begin{array}{c}\text { Year } \\
\text { significant } \\
\text { in original } \\
\text { study? }\end{array}$ & $\begin{array}{l}\text { Year } \\
\text { significant } \\
\text { in new } \\
\text { GLM? }\end{array}$ & $\begin{array}{l}\text { Terms included in best } \\
\text { GLS model }\end{array}$ & B & $p$ & $n$ & $\begin{array}{l}\text { Delta } \\
\text { AIC }\end{array}$ & $\begin{array}{c}\text { Change } \\
\text { with } \\
\text { GLS? }\end{array}$ & \\
\hline $\begin{array}{l}\text { Euphorbia } \\
\text { peplus }\end{array}$ & Yes & No & $\begin{array}{l}\text { Year } \\
\text { Moisture } \\
\text { Year } \times \text { Moisture }\end{array}$ & $\begin{array}{r}0.0067 \\
22.5306 \\
-0.0115\end{array}$ & $\begin{array}{l}0.051 \\
\underline{0.017} \\
\underline{\mathbf{0 . 0 1 6}}\end{array}$ & 68 & 3.5 & Yes & $\begin{array}{l}\text { Adding climate } \\
\text { data reveals a } \\
\text { significant effect } \\
\text { of year }\end{array}$ \\
\hline $\begin{array}{l}\text { Medicago } \\
\text { lupulina }\end{array}$ & No & No & Year & -0.0001 & 0.898 & 57 & -1.98 & No & \\
\hline $\begin{array}{l}\text { Polycarpon } \\
\text { tetraphyllum }\end{array}$ & No & $\begin{array}{c}\text { Yes } \\
\text { (Arid Region) }\end{array}$ & $\begin{array}{l}\text { Year } \\
\text { Temperature } \\
\text { Year } \times \text { Temperature }\end{array}$ & $\begin{array}{r}-0.004 \\
-23.0284 \\
0.0119 \\
\end{array}$ & $\begin{array}{c}0.759 \\
0.5 \\
0.5\end{array}$ & 49 & -3.81 & No & \\
\hline $\begin{array}{l}\text { Trifolium } \\
\text { glomeratum }\end{array}$ & No & No & $\begin{array}{l}\text { Year } \\
\text { Temperature }\end{array}$ & $\begin{array}{l}0.0002 \\
0.0175\end{array}$ & $\begin{array}{l}0.8291 \\
0.1643\end{array}$ & 29 & -1.31 & No & \\
\hline Trifolium dubium & $N / A$ & No & $\begin{array}{l}\text { Year } \\
\text { Temperature }\end{array}$ & $\begin{array}{c}0.002 \\
0.0156\end{array}$ & $\frac{\mathbf{0 . 0 2 7}}{0.153}$ & 25 & 2.28 & Yes & \\
\hline
\end{tabular}


Table S6. Results for the best fitting models to explain variation in plant height for each species introduced to New Zealand. Delta AIC is calculated in reference to the null model. Negative Delta AIC's indicate that the null model was better than the best fitting model. Change with GLS?' refers to if the best fitting model in the updated GLS approach (including climate and coordinate data), gave a different significant effect for the year (or year $\mathrm{x}$ climate term).

\begin{tabular}{|c|c|c|c|c|c|c|c|c|c|}
\hline Species & $\begin{array}{c}\text { Year } \\
\text { significant } \\
\text { in original } \\
\text { study? }\end{array}$ & $\begin{array}{c}\text { Year } \\
\text { significant in } \\
\text { new GLM? }\end{array}$ & $\begin{array}{l}\text { Terms included in best } \\
\text { GLS model }\end{array}$ & $B$ & $p$ & $n$ & $\begin{array}{l}\text { Delta } \\
\text { AIC }\end{array}$ & $\begin{array}{l}\text { Change } \\
\text { with } \\
\text { GLS? }\end{array}$ & $\begin{array}{c}\text { Explanation of } \\
\text { change with GLS }\end{array}$ \\
\hline $\begin{array}{l}\text { Acaena } \\
\text { agnipila }\end{array}$ & No & No & $\begin{array}{l}\text { Year } \\
\text { Temperature } \\
\text { Moisture } \\
\text { Year } \times \text { Moisture }\end{array}$ & $\begin{array}{r}-0.014 \\
0.041 \\
-32.627 \\
0.017\end{array}$ & $\begin{array}{l}\underline{0.022} \\
\underline{0.002} \\
\underline{0.014} \\
\underline{0.014}\end{array}$ & 78 & 9.80 & Yes & $\begin{array}{l}\text { Adding climate } \\
\text { reveals significant } \\
\text { effect of year }\end{array}$ \\
\hline $\begin{array}{l}\text { Cerastium } \\
\text { glomeratum }\end{array}$ & Yes & Yes & Year & -0.003 & 0.181 & 129 & 114.83 & Yes & $\begin{array}{l}\text { Adding coordinates } \\
\text { removes } \\
\text { significance of Year }\end{array}$ \\
\hline $\begin{array}{l}\text { Carex } \\
\text { longebrachiata }\end{array}$ & No & No & Year & -0.001 & 0.653 & 35 & -1.81 & No & \\
\hline $\begin{array}{l}\text { Euphorbia } \\
\text { peplus }\end{array}$ & No & No & Year & -0.001 & 0.446 & 166 & 2.44 & No & \\
\hline $\begin{array}{l}\text { Myosotis } \\
\text { arvensis }\end{array}$ & No & No & Year & 0.001 & 0.482 & 91 & 31.07 & No & \\
\hline Poa annua & No & No & Year & -0.002 & 0.115 & 28 & 0.51 & No & \\
\hline $\begin{array}{l}\text { Plantago } \\
\text { coronopus }\end{array}$ & No & No & $\begin{array}{l}\text { Year } \\
\text { Temperature } \\
\text { Moisture } \\
\text { Year } \times \text { Temperature } \\
\text { Year } \times \text { Moisture } \\
\text { Temperature } \times \text { Moisture }\end{array}$ & $\begin{array}{r}0.014 \\
0.506 \\
31.452 \\
0.000 \\
-0.013 \\
-0.306\end{array}$ & $\begin{array}{r}0.222 \\
0.663 \\
\underline{0.015} \\
0.857 \\
\underline{\mathbf{0 . 0 3 7}} \\
\underline{0.001} \\
\end{array}$ & 155 & 7.17 & Yes & $\begin{array}{l}\text { Adding climate and } \\
\text { coordinate reveals } \\
\text { significant } \\
\text { interaction effect } \\
\text { of year with climate. }\end{array}$ \\
\hline
\end{tabular}




\begin{tabular}{|c|c|c|c|c|c|c|c|c|c|}
\hline $\begin{array}{l}\text { Ranunculus } \\
\text { parviflorus }\end{array}$ & No & No $(0.08)$ & Year & -0.002 & 0.229 & 101 & -0.31 & No & \\
\hline Silene gallica & No & No & Year & -0.002 & 0.130 & 198 & 3.46 & No & \\
\hline $\begin{array}{l}\text { Solenogyne } \\
\text { gunnii }\end{array}$ & No & No & Year & -0.001 & 0.346 & 57 & 19.18 & No & \\
\hline $\begin{array}{l}\text { Stachys } \\
\text { arvensis }\end{array}$ & No & No & $\begin{array}{l}\text { Year } \\
\text { Temperature } \\
\text { Moisture } \\
\text { Year } \times \text { Temperature } \\
\text { Year } \times \text { Moisture } \\
\text { Temperature } \times \text { Moisture }\end{array}$ & $\begin{array}{r}0.058 \\
0.306 \\
91.510 \\
0.000 \\
-0.052 \\
0.587\end{array}$ & $\begin{array}{r}\leq 0.001 \\
0.763 \\
\underline{0.000} \\
0.415 \\
\leq 0.001 \\
\underline{0.002}\end{array}$ & 72 & 1.79 & Yes & $\begin{array}{l}\text { Adding climate and } \\
\text { coordinate reveals } \\
\text { significant } \\
\text { interaction effect } \\
\text { of year with climate }\end{array}$ \\
\hline $\begin{array}{l}\text { Trifolium } \\
\text { dubium }\end{array}$ & No & No & $\begin{array}{l}\text { Year } \\
\text { Moisture }\end{array}$ & $\begin{array}{r}-0.001 \\
0.155\end{array}$ & $\begin{array}{l}0.481 \\
0.381\end{array}$ & 111 & 4.01 & No & \\
\hline
\end{tabular}


Table S7. Results for the best fitting models to explain variation in leaf area for each species introduced to New Zealand. Delta AIC is calculated in reference to the null model. Negative Delta AIC's indicate that the null model was better than the best fitting model. Change with GLS?' refers to if the best fitting model in the updated GLS approach (including climate and coordinate data), gave a different significant effect for the year (or year $\mathrm{x}$ climate term).

\begin{tabular}{|c|c|c|c|c|c|c|c|c|c|}
\hline Species & $\begin{array}{c}\text { Year } \\
\text { significant } \\
\text { in original } \\
\text { study? }\end{array}$ & $\begin{array}{c}\text { Year } \\
\text { significant } \\
\text { in new } \\
\text { GLM? }\end{array}$ & $\begin{array}{c}\text { Terms included in best } \\
\text { GLS model }\end{array}$ & $B$ & $p$ & $n$ & $\begin{array}{l}\text { Delta } \\
\text { AIC }\end{array}$ & $\begin{array}{c}\text { Change } \\
\text { with } \\
\text { GLS? }\end{array}$ & $\begin{array}{l}\text { Explanation of } \\
\text { change with GLS }\end{array}$ \\
\hline Acaena agnipila & No & No & $\begin{array}{l}\text { Year } \\
\text { Temperature }\end{array}$ & $\begin{array}{l}0.002 \\
0.027\end{array}$ & $\begin{array}{l}0.156 \\
0.105\end{array}$ & 82 & 5.1 & No & \\
\hline $\begin{array}{l}\text { Cerastium } \\
\text { glomeratum }\end{array}$ & No & No & $\begin{array}{l}\text { Year } \\
\text { Temperature } \\
\text { Moisture } \\
\text { Year } \times \text { Temperature } \\
\text { Year } \times \text { Moisture }\end{array}$ & $\begin{array}{r}0.089 \\
7.673 \\
41.135 \\
-0.004 \\
-0.021\end{array}$ & $\begin{array}{l}\frac{0.004}{0.005} \\
\frac{0.038}{0.005} \\
0.037 \\
\end{array}$ & 139 & 11.27 & Yes & \\
\hline $\begin{array}{l}\text { Euphorbia } \\
\text { peplus }\end{array}$ & No & No $(0.076)$ & $\begin{array}{l}\text { Year } \\
\text { Moisture }\end{array}$ & $\begin{array}{l}-0.000 \\
-0.264 \\
\end{array}$ & $\begin{array}{l}0.729 \\
0.079\end{array}$ & 141 & 40.12 & No & \\
\hline $\begin{array}{l}\text { Lythrum } \\
\text { hyssopifolia }\end{array}$ & No & No & $\begin{array}{l}\text { Year } \\
\text { Temperature } \\
\text { Year } \times \text { Temperature }\end{array}$ & $\begin{array}{r}0.035 \\
3.803 \\
-0.002 \\
\end{array}$ & $\begin{array}{l}0.084 \\
0.095 \\
0.087\end{array}$ & 45 & 3.35 & No & \\
\hline $\begin{array}{l}\text { Medicago } \\
\text { lupulina }\end{array}$ & No & Yes & $\begin{array}{l}\text { Year } \\
\text { Temperature }\end{array}$ & $\begin{array}{r}-0.005 \\
0.042\end{array}$ & $\underline{0.005}$ & 56 & 25.15 & No & \\
\hline Melilotus indicus & Yes & No & Year & 0.0002 & 0.866 & 68 & 3.24 & No & \\
\hline $\begin{array}{l}\text { Myosotis } \\
\text { arvensis }\end{array}$ & No & No & $\begin{array}{l}\text { Year } \\
\text { Temperature } \\
\text { Moisture }\end{array}$ & $\begin{array}{l}0.004 \\
0.049 \\
0.540\end{array}$ & $\begin{array}{l}\underline{0.023} \\
0.082 \\
\underline{0.009} \\
\end{array}$ & 85 & 4.66 & Yes & $\begin{array}{l}\text { Adding climate } \\
\text { and coordinates } \\
\text { changes year to } \\
\text { significant }\end{array}$ \\
\hline $\begin{array}{l}\text { Persicaria } \\
\text { maculosa }\end{array}$ & No & No & Year & 0.863 & 0.842 & 42 & 12.36 & No & \\
\hline $\begin{array}{l}\text { Ranunculus } \\
\text { parviflorus }\end{array}$ & No & No & $\begin{array}{l}\text { Year } \\
\text { Moisture }\end{array}$ & $\begin{array}{r}-0.0008 \\
0.9493\end{array}$ & $\begin{array}{l}0.633 \\
0.106\end{array}$ & 96 & 5.46 & No & \\
\hline
\end{tabular}




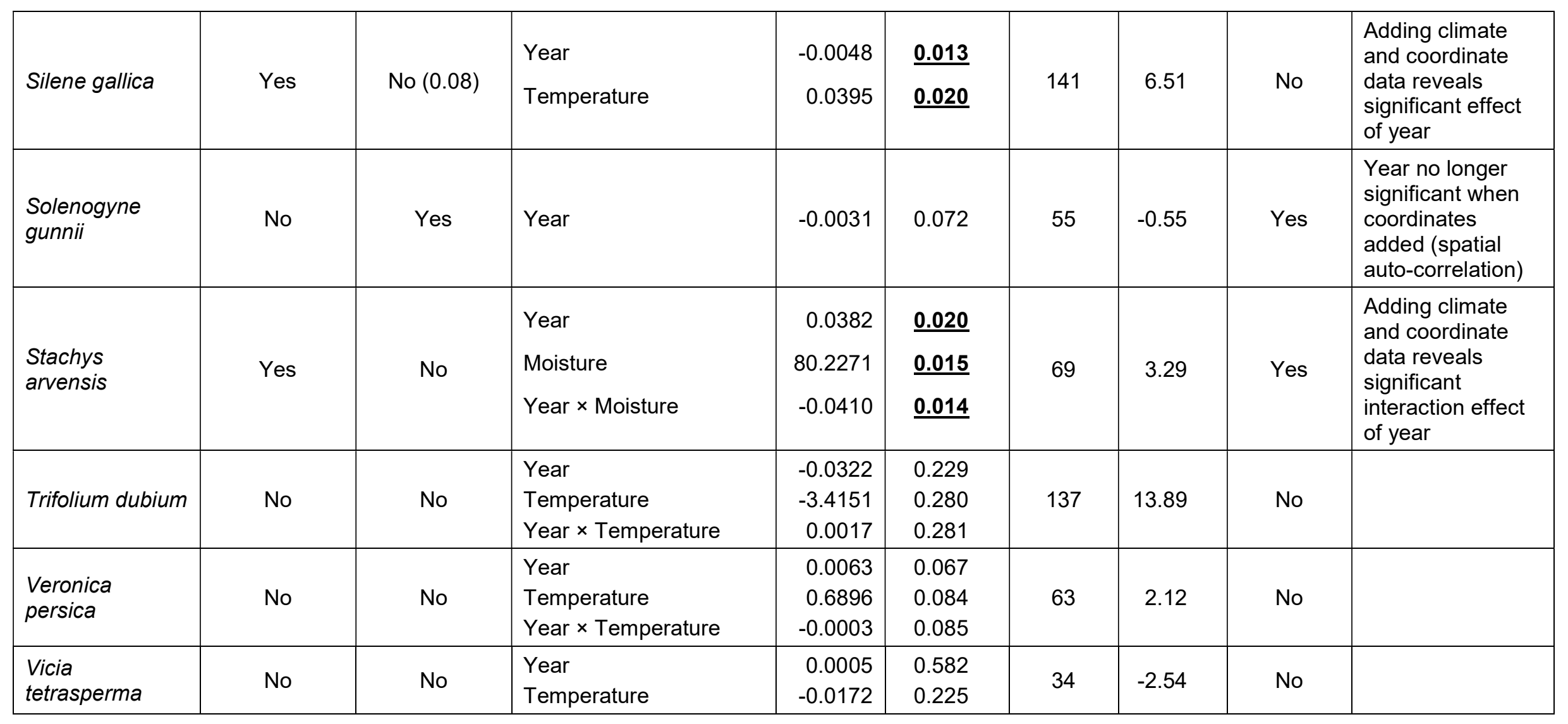


Table S8. Results for the best fitting models to explain variation in leaf shape for each species introduced to New Zealand. Delta AIC is calculated in reference to the null model. Negative Delta AIC's indicate that the null model was better than the best fitting model Change with GLS?' refers to if the best fitting model in the updated GLS approach (including climate and coordinate data), gave a different significant effect for the year (or year $\mathrm{x}$ climate term).

\begin{tabular}{|c|c|c|c|c|c|c|c|c|c|}
\hline Species & $\begin{array}{c}\text { Year } \\
\text { significant in } \\
\text { original } \\
\text { study? }\end{array}$ & $\begin{array}{c}\text { Year } \\
\text { significant } \\
\text { in new } \\
\text { GLM? }\end{array}$ & $\begin{array}{l}\text { Terms included in } \\
\text { best GLS model }\end{array}$ & $B$ & $p$ & $n$ & $\begin{array}{l}\text { Delta } \\
\text { AIC }\end{array}$ & $\begin{array}{c}\text { Change } \\
\text { with } \\
\text { GLS? }\end{array}$ & $\begin{array}{c}\text { Explanation of } \\
\text { change with GLS }\end{array}$ \\
\hline $\begin{array}{l}\text { Acaena } \\
\text { agnipila }\end{array}$ & No & No & $\begin{array}{l}\text { Year } \\
\text { Moisture }\end{array}$ & $\begin{array}{l}-0.0002 \\
-0.0386\end{array}$ & $\begin{array}{l}0.402 \\
0.322\end{array}$ & 82 & -2.42 & No & \\
\hline $\begin{array}{l}\text { Cerastium } \\
\text { glomeratum }\end{array}$ & No & No & $\begin{array}{l}\text { Year } \\
\text { Temperature } \\
\text { Moisture } \\
\text { Year } \times \text { Moisture }\end{array}$ & $\begin{array}{r}-0.0032 \\
-0.0276 \\
-7.0471 \\
0.0035\end{array}$ & $\begin{array}{r}0.157 \\
<0.001 \\
0.114 \\
0.119\end{array}$ & 139 & 6.68 & No & \\
\hline $\begin{array}{l}\text { Euphorbia } \\
\text { peplus }\end{array}$ & No & No & $\begin{array}{l}\text { Year } \\
\text { Temperature }\end{array}$ & $\begin{array}{l}-0.00006 \\
-0.0060\end{array}$ & $\begin{array}{l}0.838 \\
0.087\end{array}$ & 141 & -4.7 & No & \\
\hline $\begin{array}{l}\text { Lythrum } \\
\text { hyssopifolia }\end{array}$ & No & No & $\begin{array}{l}\text { Year } \\
\text { Temperature } \\
\text { Year } \times \text { Temperature }\end{array}$ & $\begin{array}{r}0.0177 \\
2.0869 \\
-0.0010\end{array}$ & $\begin{array}{l}0.028 \\
\underline{0.022} \\
\underline{0.023} \\
\end{array}$ & 45 & 1.6 & No & \\
\hline $\begin{array}{l}\text { Medicago } \\
\text { lupulina }\end{array}$ & No & No & $\begin{array}{l}\text { Year } \\
\text { Temperature } \\
\text { Year } \times \text { Temperature }\end{array}$ & $\begin{array}{r}0.0129 \\
1.5677 \\
-0.0008\end{array}$ & $\begin{array}{l}\frac{0.040}{0.029} \\
\underline{0.030}\end{array}$ & 56 & 1.31 & Yes & $\begin{array}{l}\text { Adding climate } \\
\text { and coordinate } \\
\text { reveals significant } \\
\text { interaction effect } \\
\text { of year. }\end{array}$ \\
\hline $\begin{array}{l}\text { Melilotus } \\
\text { indicus }\end{array}$ & No & No & Year & 0.0004 & 0.437 & 68 & -1.37 & No & \\
\hline $\begin{array}{l}\text { Myosotis } \\
\text { arvensis }\end{array}$ & Yes & No & $\begin{array}{l}\text { Year } \\
\text { Moisture }\end{array}$ & $\begin{array}{l}0.0017 \\
0.1496\end{array}$ & $\frac{0.010}{0.153}$ & 89 & 2.3 & Yes & $\begin{array}{l}\text { Adding climate } \\
\text { and coordinates } \\
\text { makes year } \\
\text { significant. }\end{array}$ \\
\hline $\begin{array}{l}\text { Persicaria } \\
\text { maculosa }\end{array}$ & No & No & $\begin{array}{l}\text { Year } \\
\text { Temperature }\end{array}$ & $\begin{array}{l}0.0257 \\
5.5770\end{array}$ & $\begin{array}{l}0.166 \\
\mathbf{0 . 0 1 0}\end{array}$ & 42 & 12.36 & Yes & $\begin{array}{l}\text { Adding climate } \\
\text { and coordinate }\end{array}$ \\
\hline
\end{tabular}




\begin{tabular}{|c|c|c|c|c|c|c|c|c|c|}
\hline $\begin{array}{l}\text { Persicaria } \\
\text { maculosa } \\
\text { (cont.) }\end{array}$ & & & $\begin{array}{l}\text { Moisture } \\
\text { Year } \times \text { Temperature } \\
\text { Year } \times \text { Moisture }\end{array}$ & $\begin{array}{r}-44.0358 \\
-0.0028 \\
0.0224\end{array}$ & $\begin{array}{l}\frac{0.004}{0.010} \\
\underline{0.004} \\
\end{array}$ & & & & $\begin{array}{l}\text { reveals significant } \\
\text { interaction effect } \\
\text { of year. }\end{array}$ \\
\hline $\begin{array}{l}\text { Ranunculus } \\
\text { parviflorus }\end{array}$ & No & No & $\begin{array}{l}\text { Year } \\
\text { Temperature }\end{array}$ & $\begin{array}{l}0.000001 \\
0.0131\end{array}$ & $\begin{array}{l}0.994 \\
0.263\end{array}$ & 96 & 15.65 & No & \\
\hline Silene gallica & Yes & Yes & Year & 0.0016 & $\underline{0.005}$ & 143 & 13.37 & No & $\begin{array}{l}\text { Year remains } \\
\text { significant }\end{array}$ \\
\hline $\begin{array}{l}\text { Solenogyne } \\
\text { gunni }\end{array}$ & No & No & Year & 0.00022 & 0.299 & 58 & -0.87 & No & \\
\hline $\begin{array}{l}\text { Stachys } \\
\text { arvensis }\end{array}$ & No & No & Year & -0.0004 & 0.335 & 69 & -7.62 & No & \\
\hline $\begin{array}{l}\text { Trifolium } \\
\text { dubium }\end{array}$ & No & No & $\begin{array}{l}\text { Year } \\
\text { Temperature } \\
\text { Year } \times \text { Temperature }\end{array}$ & $\begin{array}{r}0.0062 \\
0.7078 \\
-0.0004\end{array}$ & $\begin{array}{l}0.249 \\
0.264 \\
0.260\end{array}$ & 137 & 12.18 & No & \\
\hline $\begin{array}{l}\text { Veronica } \\
\text { persica }\end{array}$ & No & No & Year & 0.0015 & 0.201 & 63 & -0.05 & No & \\
\hline $\begin{array}{l}\text { Vicia } \\
\text { tetrasperma }\end{array}$ & No & No & $\begin{array}{l}\text { Year } \\
\text { Moisture }\end{array}$ & $\begin{array}{l}-0.0003 \\
-0.0201\end{array}$ & $\begin{array}{l}0.616 \\
0.892\end{array}$ & 35 & -3.74 & No & \\
\hline
\end{tabular}




\title{
4. Do climate niche shifts predict trait change through time in introduced plants?
}

\begin{abstract}
Introduced plants often undergo realised niche shifts, occupying climates distinctly different from those in their native range. This change in the abiotic environment may exert novel selection pressures on introduced plants, which could lead to adaptive trait change through time. This study aims to test if the direction and magnitude of realised climate niche shifts, for 34 plant species recently introduced to Australia and New Zealand, could predict the rate of morphological trait change through time in the introduced range. I re-analysed morphological change through time in key plant traits (height, leaf area, and leaf shape) for approximately 4000 herbarium specimens. Climate niche shifts were then quantified for each of the introduced species. The overall rate of trait change through time for each trait was then predicted from the magnitude of each species climate niche shift, in a meta-analysis. Species climate niche shifts did not effectively predict plant trait change through time, indicating that climate may not be the predominant driver of trait change in introduced plants to Australia and New Zealand. Alternatively, the combined noise and the mismatch in scales that may arise when combining these two methods could mask any underlying patterns in plant trait responses to the new environment. While the use of herbarium specimens and species distribution models may individually provide useful tools to explore species responses to shifts in geographic range and climate, the two approaches did not combine to provide a consistent perspective on how these processes affect species that have expanded into introduced ranges where they may experience novel abiotic conditions.
\end{abstract}




\subsection{Introduction}

Non-native plants can often become successfully established in a new range, despite environmental conditions being largely different from the native range in which they originate (Broennimann et al. 2007; Gallagher et al. 2010; Bocsi et al. 2016). This change in the abiotic environment can expose them to novel selection pressures, leading to rapid evolution in the introduced range (Dlugosch \& Parker 2008; Colautti \& Barret 2013; Gallien et al. 2016). If rapid evolution in the introduced range is driven primarily by a change in environmental niche, then it may be possible to use climate niche shifts to predict trait change through time in introduced species.

Species distribution models (SDMs) are becoming increasingly recognised as a tool to understand the linkage between species distributions and their underlying biological mechanisms (Elmendorf \& Moore 2008; Thuiller et al. 2009; Wittmann et al. 2016). Thuiller et al. (2009) found that suitability scores from SDM models could be used to predict plant performance through measures of plant functional traits in a number of alpine plants. Brambilla \& Ficetola (2012) found suitability scores from correlative SDMs to positively correlate with reproductive output in passerine birds. If suitability scores generated from SDMs can predict an organism's fitness, via functional traits or reproductive output, then a niche shift may correspond to lower predicted suitability scores and an increase selection pressure to adapt, when introduced to a new environment. However, this has never been formally tested.

In the previous chapter (Chapter 3) I found evidence for morphological change through time for 32 out of 94 trait-species combinations, in plants introduced to Australia and New Zealand. This was based on functional trait measurements (plant height, leaf area, leaf shape, and leaf mass per unit area) taken from historic herbarium specimens, sampled through time (Buswell 2009; Buswell et al. 2011). The rate and direction of trait change through time, as well as varying rates of trait change along different climate gradients, was highly variable between species. One 
possible reason for this is that species originated from different native ranges with distinct climates. Thus, the climate in the introduced range could have had a species specific response on the rate and direction of trait change through time. If this is the case, then the rate of trait change through time would be expected to increase with a greater magnitude of climate niche shift in the introduced range. The aim of this study is to examine if climatic niche shifts calculated from species distribution data can effectively predict morphological change through time in introduced plants. Due to the correlative nature of this study, niche shifts will refer to a shift in the climate space occupied by a species (i.e., a shift in the realised niche). This is the first study to simultaneously assess trait change in multiple introduced species, in relation to their climate niche shift.

Species distribution modelling has been widely used to predict the potential future distribution that a species could achieve when introduced to a new range (Peterson \& Vieglais 2001; Peterson 2003; Thuiller et al. 2005b). This approach assumes niche conservatism, that a species will retain its niche-related ancestral ecological requirements through space and time (Wiens \& Graham 2005). However, the evidence for niche conservatism is mixed with different studies showing evidence for both niche conservatism (Peterson 2011; Petitpierre et al. 2012) and niche differentiation (Fitzpatrick et al. 2007; Broennimann et al. 2007; Gallagher et al. 2010; Bocsi et al 2016). These different findings could be due to methodological differences in quantifying niche shifts (Warren et al. 2008; Guisan et al. 2014). Crucially, the consideration of the total climate space available to the species in each range, rather than simply the climate space occupied, has been highlighted to be of key importance when quantifying niche shifts (Mandle et al. 2010; Peterson et al. 2011; Broennimann et al. 2012; Petitpierre et al. 2012). Accounting for the availability of climate conditions in each range prevents differences in species distributions from being interpreted as a niche shift, when they are simply due to differences in the climate space available (Soberon \& Peterson 2011; Guisan et al. 2014). 
A large scale study of 50 invasive plants found niche shifts to be rare, when only analog climate (climate space that is shared between both ranges) was accounted for (Petitpierre et al. 2012). Under these conditions, niche expansion into analog climate space is described as 'true' niche shift, which could arise through rapid evolution or a change in biotic interactions (Petitpierre et al. 2012; Guisan et al. 2014). In contrast, niche shifts that are detected when including non-analog climate space are described as 'pseudo-shifts', as it is not possible to tell if they represent a true niche shift or simply the filling of niche space for which a species is already pre-adapted (i.e., representing no change in the fundamental niche; Petitpierre et al. 2012).

Gallagher et al. (2010) found plant species introduced to Australia to frequently occupy climate conditions outside of their native range (20 of 26 species), but were unable to link these niche shifts to any key plant trait, or characteristics relating to their introduction or distribution. However, their approach only looked to quantify the degree of niche overlap, and calculated niche shifts including non-analog climate space, which would likely over estimate niche shift magnitude (Petitpierre et al. 2012). Considering shifts into non-analog climates is problematic as most studies examining niche shifts do not include complementary information of the biology of the species (Guisan et al. 2014). However, by relating trait change through time to niche shifts into both analog and non-analog climate space, this may allow for greater understanding into what is driving niche evolution.

When quantifying niche shifts with the inclusion of non-analog climates, niche shifts would be expected to be detected more frequently and to a greater magnitude, than when assessed using only analog climate space. However, the way that these different approaches may predict trait change through time is unclear. If a 'true' niche shift is detected using only analog climates, then this may be due to expansion of the fundamental niche via rapid evolution, and thus may be more likely to correlate with trait change through time. On the other hand, a niche shift detected when including non-analog climates will likely reveal greater climatic differences between the ranges, 
in which case there would be a greater selective pressure expected to drive trait change through time in the introduced range.

The aim of this study is to examine if climatic niche shifts calculated from species distribution data can effectively predict morphological change through time in introduced plants. To do this I first test for rates of niche shifts in 34 plant species introduced to Australia and New Zealand (considering both analog and non-analog climate space). I then ask: i) does the magnitude of a species climatic niche shift predict the rate of change through time in certain traits?, ii) does the direction and magnitude of a climate niche centroid shift predict the direction and rate of trait change through time?, iii) do herbarium specimens that were sampled in areas of novel climate space (relative to the home range) change at a faster rate than those sampled from areas of climate space shared with the native range, and iv) do niche shifts calculated across shared (analog) or novel (non-analog) climate spaces affect predictions of trait change through time. 


\subsection{Methods}

\subsubsection{Data Collection}

\subsubsection{Herbarium specimen trait and location data}

Plant trait data was compiled for 19 species introduced to Australia and 16 species introduced to New Zealand. Trait measurements were taken from herbarium specimens in Buswell (2009) and Buswell et al. (2011). These traits comprised of plant height, leaf area and leaf shape. See previous chapter for details on how traits were measured. I added to this dataset by georeferencing the herbarium specimens using the information available on each herbarium record sheet, as either coordinates or as a written description of the location. Where both coordinates and a description were given, they were cross referenced to check if they matched. In cases where they did not match, the written description was used preferentially. Google Earth (Google Earth 2014), was used to locate written descriptions and assign them coordinate values. Herbarium specimens that did not have information on location, or if this was not precise enough to determine its location within a $10 \mathrm{~km}$ radius, were omitted from the study. Coastal records that fell outside the climate grid cells were assigned the value of the closest cell on land (up to a maximum of $20 \mathrm{~km}$ away). Each species was required to have a minimum of 25 geo-referenced specimens to be included in the analysis. See Figure 7 for outline of the full methodological procedure taken.

\subsubsection{Climate data}

Climate data for 35 bioclimatic variables, at 10' spatial resolution (this gives cell dimensions of approximately $18.5 \mathrm{~km}$ East-West, and $14.2 \mathrm{~km}$ North-South at $40^{\circ}$ Latitude), were obtained from the CliMond dataset (V1.2, Kriticos et al. 2012). This was deemed to be the resolution that best reflected that accuracy of the herbarium record locations. The first 19 core bioclimatic variables are derived from weekly measures of minimum temperature $\left({ }^{\circ} \mathrm{C}\right)$, maximum temperature $\left({ }^{\circ} \mathrm{C}\right)$ and 
precipitation $(\mathrm{mm})$, variables taken from a network of meteorological stations. The remaining 16 bioclimatic variables are derived from modelled monthly values of soil moisture index and solar radiation $\left(\mathrm{W} \mathrm{m} \mathrm{m}^{-2} \mathrm{~d}^{-1}\right)$. These are combined to give mean climate values collected over 30 years, from 1961 to 1990 , centred on 1975. Climate data was extracted for each georeferenced herbarium specimen using the Raster package in $\mathrm{R}$.

To avoid over-fitting models with multiple variables, only two climate variables that were considered to be strong potential predictors of plant morphological change through time were used. These were selected based on their known potential influence on plant growth, and derived from different underlying climatic factors (i.e., a climate variable for water moisture and a climate variable for temperature). A PCA analysis was performed in order to select two variables that: i) captured a large amount of variance across the first 2 axis of the PCA (Figure 6, Chapter 3), ii) were perceived to have differing effect on plant growth. The first two axis of the PCA analysis explained $72.7 \%$ of the total variation in climate for Australian herbarium records, and $73.7 \%$ for New Zealand records (Table S1). Through this approach the soil moisture index of the driest 3 months of the year (PCA axis 1; Bio33, hereafter referred to as Moisture), and the mean temperature of the warmest quarter (PCA axis 2; Bio10, hereafter referred to as Temperature; ${ }^{\circ} \mathrm{C}$ ), were selected. The soil moisture index describes a value from zero (oven dry) to one (field capacity), values greater than one are possible and represent excess moisture above field capacity (Kriticos et al. 2012). Although other climate datasets are available at finer resolutions (e.g., Hijmans 2005), the CliMond dataset was preferentially used as it included climate variables that would otherwise be unavailable (i.e., moisture index). 


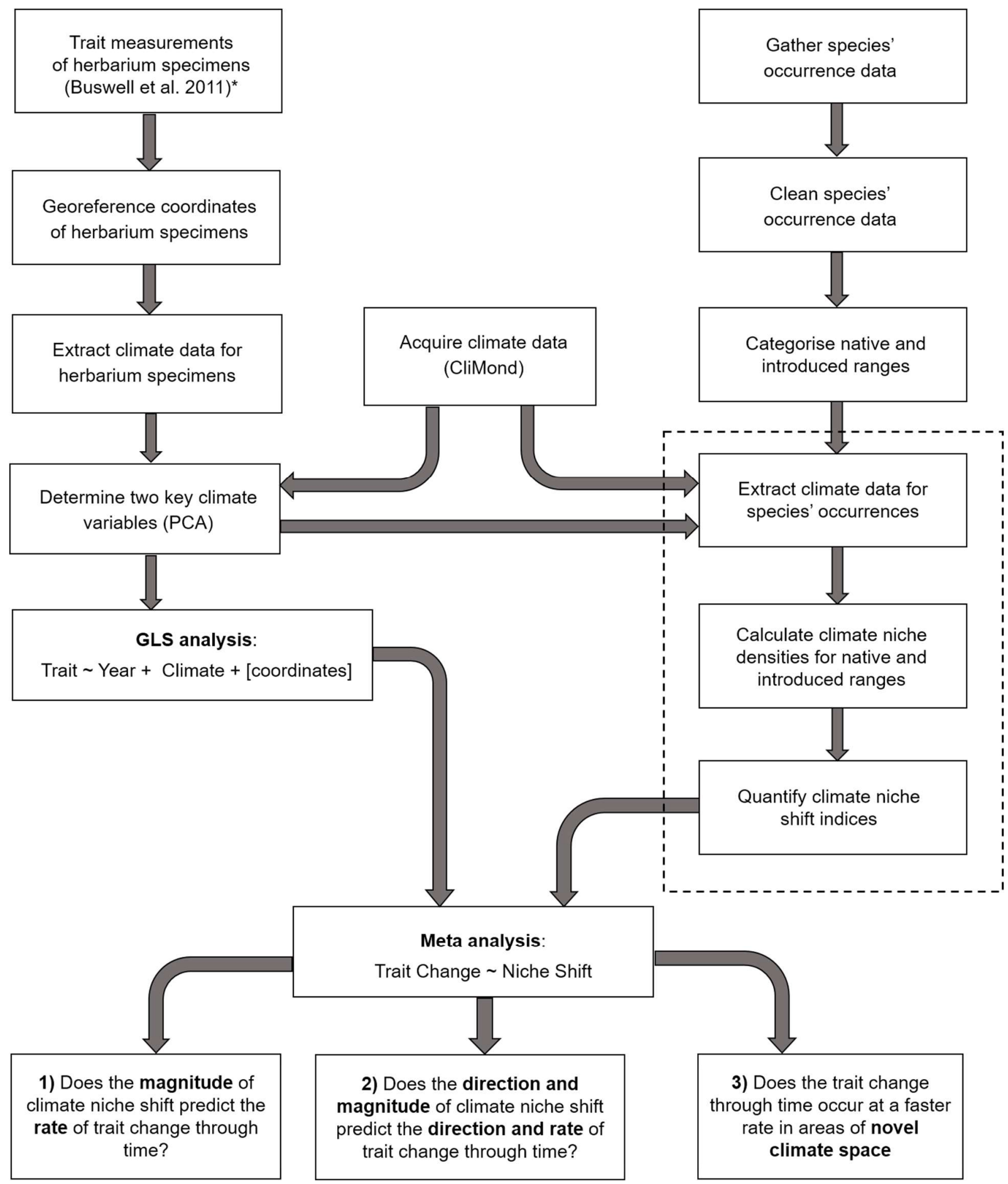

Figure 7. Flow diagram of methods used to collate data and address the three key questions of the study. The dashed box represents a more complex part of the process for quantifying climate niche shift indices, outlined in greater detail in Figure 2.

*Indicates data that was already collected and available, prior to researching this thesis. 


\subsubsection{Species distribution data}

Occurrence records for the native and introduced ranges were collated from the Global Biodiversity Information Facility (GBIF, http://www.gbif.org/), New Zealand's virtual herbarium (http://www.virtualherbarium.org.nz/), Australia's virtual herbarium (http://avh.chah.org.au/), and from georeferenced herbarium records already collected. The native range for each species was identified from a global compendium of weeds (Randall 2012), Delivering Alien Invasive Species Inventories for Europe (DAISIE, http://www.europe-aliens.org/default.do), the U.S national plant Germplasm System (http://www.ars-grin.gov/npgs/index.html), the Centre for Agriculture and Bioscience International Invasive Species Compendium (http://www.cabi.org/isc/), and the Global Invasive Species Database, http://www.issg.org/database/welcome/). When conflicting information was presented, a species' status in neighbouring countries was considered in assessing the likelihood of that species being native or non-native in a given country. 


\section{Box 1: Glossary of terms related to niche shifts}

\section{Calculating Climate Niches:}

1. Species occurrence densities: the kernel smoothed density of species' presence points occurring in gridded cells in climate space, re-scaled to range between 0 and 1 .

2. Environmental occurrence densities: the kernel smoothed density of background points occurring in gridded cells in climate space.

3. Species occupancy: The kernel smoothed density of a species occurrences in climate space, corrected for the prevalence of climate space available (environmental occurrence density).

4. Analog climate: Background climate space that is shared between the native and introduced ranges (i.e., where the environmental occurrence densities for each range overlap).

5. Non-analog climate: Background climate space that is not shared between the native and introduced ranges (i.e., where the environmental occurrence densities for each range do not overlap).

6. Marginal climate space: The marginal climate space available in each range, calculated from percentiles of the environmental occurrence densities (at $75,80,85$, 90, 95 and 99\%).

\section{Quantifying Niche Shifts:}

7. Niche expansion: The proportion of a species' occurrence densities in the introduced range niche (ranges from that do no overlap with the native niche (ranges from 0-1).

8. Niche unfilling: The proportion of a species' occurrence densities in the native range niche that do no overlap with the introduced range niche (ranges from 0-1).

9. Niche stability: The proportion of species occurrence densities in the introduced range niche that do overlap with the native niche (ranges from 0-1).

10. Total niche change: the sum of niche expansion + niche unfilling.

11. Schoener's D: A measure of the degree of overlap of species occupancy between the native and introduced ranges. It ranges from 0 (no overlap) to 1 (complete overlap).

12. Niche centroid shift: The difference in the weighted mean species occurrence densities between ranges, along a respective axis of climate space. 


\subsubsection{Calculating native and introduced range climate niches}

A common approach used for many SDM algorithms is to use the true presence data of a species' occurrence, along with randomly generated pseudo-absence data, to describe the relative likelihood of a species occurring in a particular climate. However, because various combinations of climates will vary in their frequency of occurrence in geographic space, or sometimes not exist at all, there may be large areas of climate space for which there is no presence or pseudoabsence data. Consequently SDM models that are extrapolated to predict suitability into novel or unavailable climate space may over or under-predict their climate niche suitability, with these error in predictions continuing into calculations of niche overlap (Warren et al. 2008). Thus it is of key importance that the climate space available in a geographic region is accounted for in any calculations of a species niche in climate space.

Rather than using presences and pseudo-absences to predict suitability scores, the climate niche was determined from species presences, while accounting for the total climate space available in that range. A buffer with a radius of $800 \mathrm{~km}$ was created around the presence points in a given range for each species. All presence and absence cells that occurred within this buffer were considered as the background environment available to that species' range.

To calculate a species' climate niche, I followed the approach outlined in Broennimann et al. (2012; Figure 8). Niches were calculated along 2 climatic axes, the first being an axis of temperature; 'the average temperature of the warmest quarter, the second as an axis of water availability; 'the average moisture index of the driest quarter'. To account for any sampling bias, point records for each species were converted into cells of presence-absence at 10 arc resolution (approx. $20 \mathrm{~km}^{2}$ ). This was done to: i) avoid any sampling of potential duplicate records (e.g., those that are recorded twice in different datasets), and ii) reduce any bias in sampling location that could arise from areas that have a greater likelihood to be sampled (e.g. near cities and roads). Real climate values were used, as opposed to PCA axis as is common in niche shift analyses 
(Broennimann et al. 2012; Petitpierre et al. 2012), as these would be more meaningful when predicting and interpreting the direction of trait change through time.

The total environmental space of both the native and introduced ranges was gridded into $100 \mathrm{x}$ 100 cells. The frequency of species occurrences and background occurrences were then converted into densities. A standard Gaussian kernel function was applied to determine the smoothed density for species occurrences, and background occurrences for each cell in climate space (Broennimann et al. 2012). This gives a smoothed envelope of a species' density of occurrence in climate space. These smoothed occurrence densities were used to calculate niche envelopes that were: i) occupied by the species, and ii) available to the species. These niche envelope thresholds were cut-off at the $99^{\text {th }}$ percentile, to mitigate potential adverse side effects of the kernel smoothing giving many very low density values. See Figure 9 for an example of how climate niches for a species' native and introduced range was determined from presence locations in geographic space (for details on each species, see Figures S9-S42). 


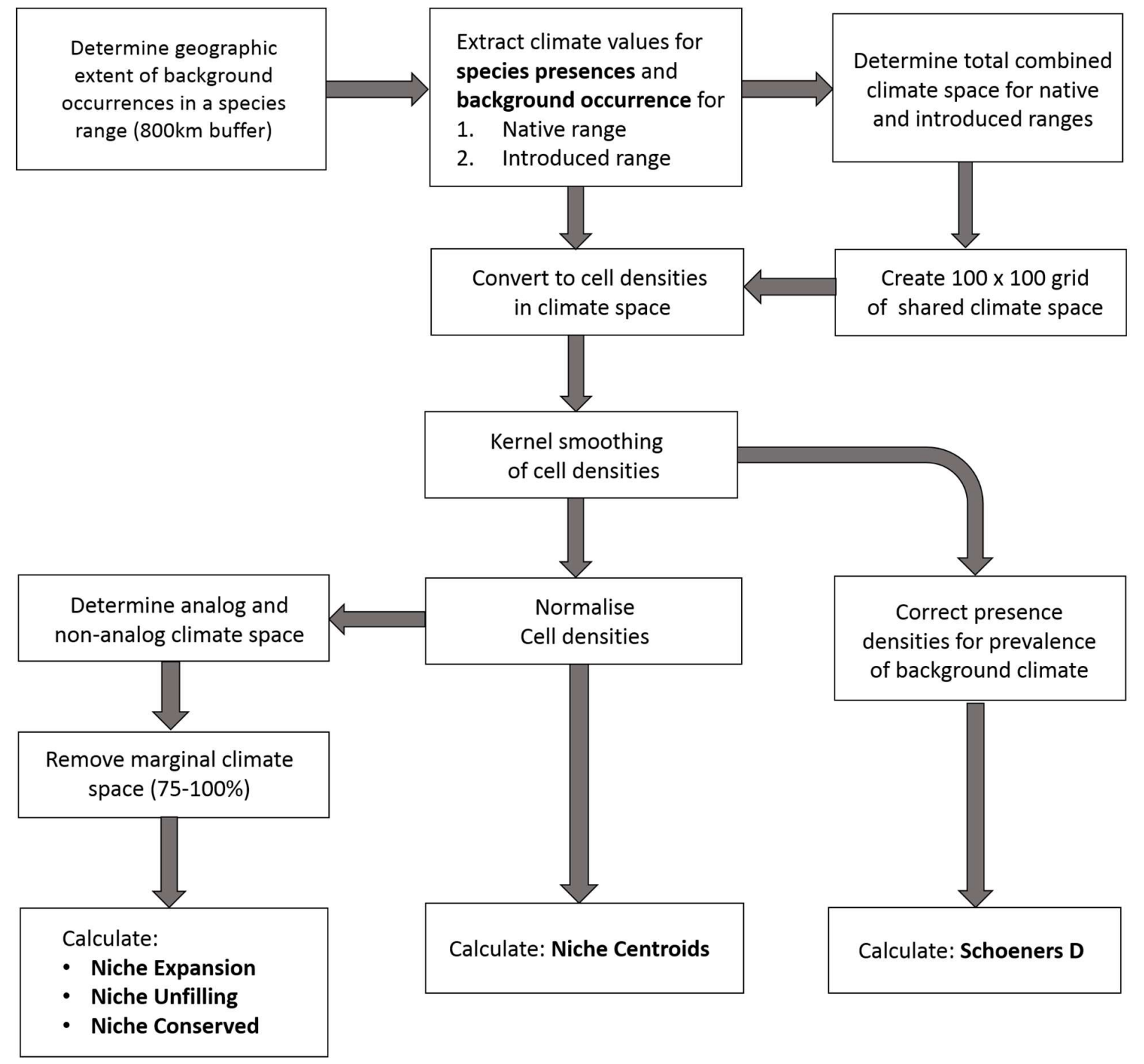

Figure 8. Flow diagram of the procedure for quantifying climate niche shift indices. 
Native Distribution

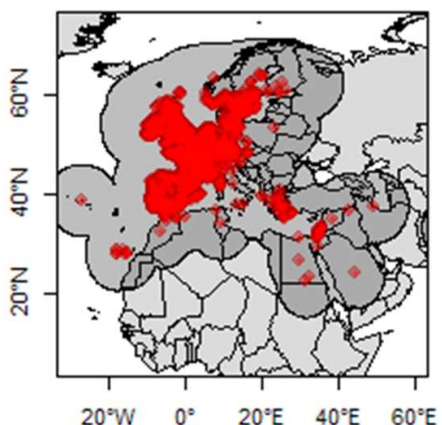

Introduced Distribution

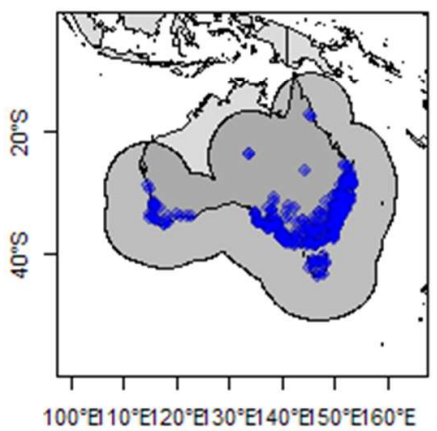

Native Climate Space

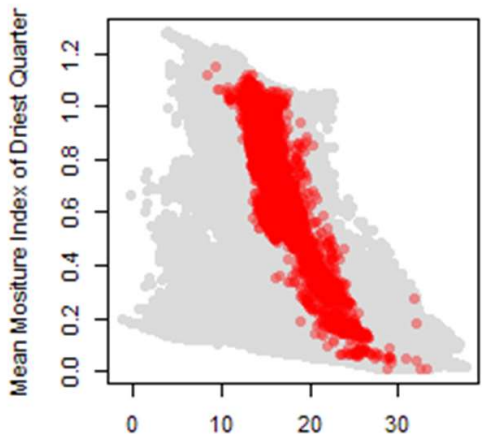

Mean Temperature of Warmest Quarter Introduced Climate Space

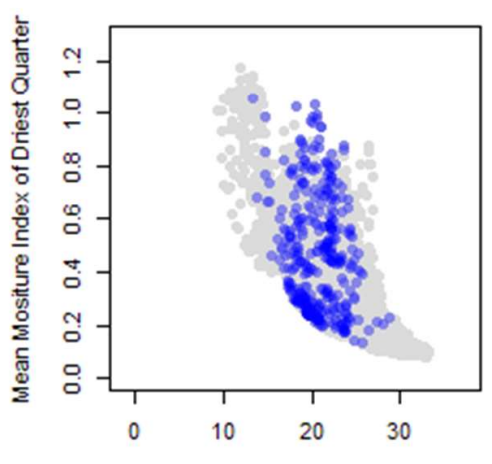

Mean Temperature of Warmest Quarter
Native Climate Densities

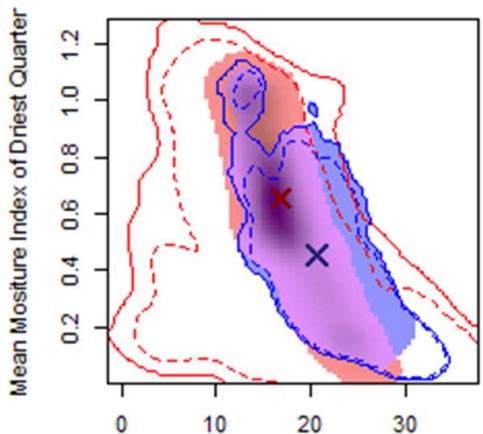

Mean Temperature of Warmest Quarter Introduced Climate Densities

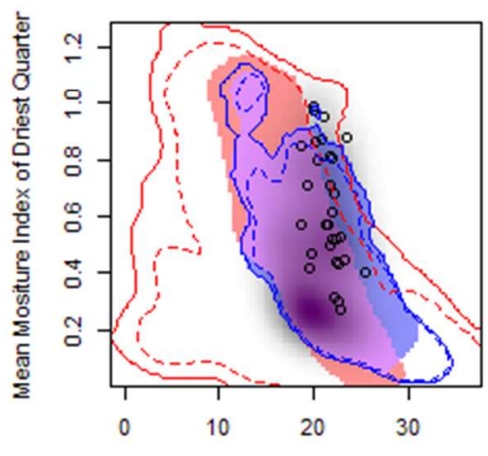

Mean Temperature of Warmest Quarter

Figure 9. Demonstration of the climatic niche calculated for the native and introduced ranges of Euphorbia peplus. Left panels: Red (native) and blue (introduced) dots represent the locations for the native and introduced locations respectively, surrounding grey shading show the $800 \mathrm{~km}$ buffer in which background climate points were taken from. Middle panels: Red and blue dots represent the location of presences in climate space, grey dots represent the background points in climate space. Right panels: Solid lines represent the extent of the available climate space at the $99^{\text {th }}$ percentile for the native range (red) and introduced range (blue), dashed lines show this at the 75th percentile; coloured areas show the extent of climate space occupied in only the native range (red; niche unfilling), the introduced range (blue; niche expansion) and their overlap (purple; niche conserved); shading (greyscale) represents the densities (species occupancy) of the present locations in climate space for the native (top) and introduced (bottom) ranges; crosses (top panel) show the niche centroid for the native (red) and introduced ranges (blue); black circles (bottom panel) show the location in climate space of the herbarium specimens used to model trait change through time. 


\subsubsection{Quantifying Niche Shifts}

\subsubsection{Schoener's D index (D)}

Schoener's $D$ was used as an index to describe the degree of niche overlap between the native and introduced range (Schoener 1968; See Box 1 for Glossary of terms). Schoener's D describes the overall match between two niches calculated in climate space, varying between 0 (no overlap) and 1 (identical overlap). The density of species occupancy was calculated for native and introduced ranges along two axis of climate space (described above), as in Broennimann et al. (2012). Species occupancy $\left(z_{i j}\right)$ for each cell was calculated from species occurrence densities $\left(o_{i j}\right)$, corrected for the background environment occurrence densities $\left(e_{i j}\right)$, as:

$$
\begin{gathered}
z_{i j}=\frac{o_{i j} / e_{i j}}{\max (o / e)} \\
\text { while } e_{i j} \neq 0 \text {, and } z_{i j}=0 \text { if } e_{i j}=0
\end{gathered}
$$

The species occupancy $\left(z_{i j}\right)$ ranges between 0 and 1 , and corresponds to the species density corrected by the environmental availability in that range. From the species occupancy, the degree of overlap (Schoener's D) can be calculated (Schoener 1970; Warren, Glor \& Turelli 2008) :

$$
D=1-\frac{1}{2}\left(\sum_{i j}\left|z_{1 i j}-z_{2 i j}\right|\right)
$$

Where $z_{1 i}$ is the species occupancy of niche one (i.e., the native range), and $z_{2 i j}$ is the species occupancy of niche two (i.e., the introduced range). The calculation of species occupancy ensures that overlap is only measured for climate space available to the species, while the use of a smooth kernel function means that the measure of overlap is independent of the resolution of the grid (Broennimann et al 2012). 


\subsubsection{Niche expansion, niche unfilling, niche stability and total niche change}

Four metrics were used to describe the magnitude and characteristics of how the niche had changed in the introduced range. These were niche expansion, niche unfilling, niche stability and total niche change. They were calculated from the proportion of species occurrence densities that overlap between the native and introduced ranges, as in Petitpierre et al. (2012). The species occurrence densities for each cell were normalized (by dividing by the maximum species occurrence density, rescaling the values to be bound between 0 and 1) to allow for comparisons between species. Niche expansion is the proportion of cell densities in the introduced range niche that occupies novel climate space. Niche unfilling is the proportion of cell densities in the native range niche that do not overlap with the introduced range. Niche stability is the proportion of cell densities in the introduced range niche that overlap with the native range (1 - niche expansion). Total niche change is the sum of niche expansion + niche unfilling. Niche expansion and total niche change were considered as two key metrics for this study, as the most relevant to quantifying the magnitude of the niche shift in the introduced range.

Niche expansion and total niche change were calculated for both analog and non-analog climates (Figure 10). Non-analog climate space is all the available background climate space for a given species' native or introduced range niche. Analog climate space is calculated as the background climate space that is shared between the native and introduced ranges (i.e., where the cell occupancies of the available climate niche overlap). Niche expansion and total niche change were then calculated for each species to include different percentile levels of marginal climate space (99\%, 95\%, 90\%, 85\%, 80\%, 75\%; Tables S12 - S45), as recommended by Guisan et al. (2014). 


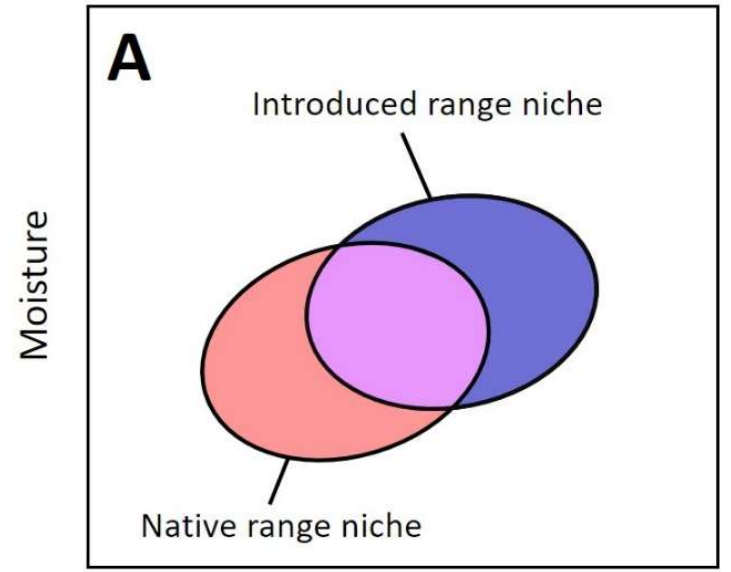

Temperature

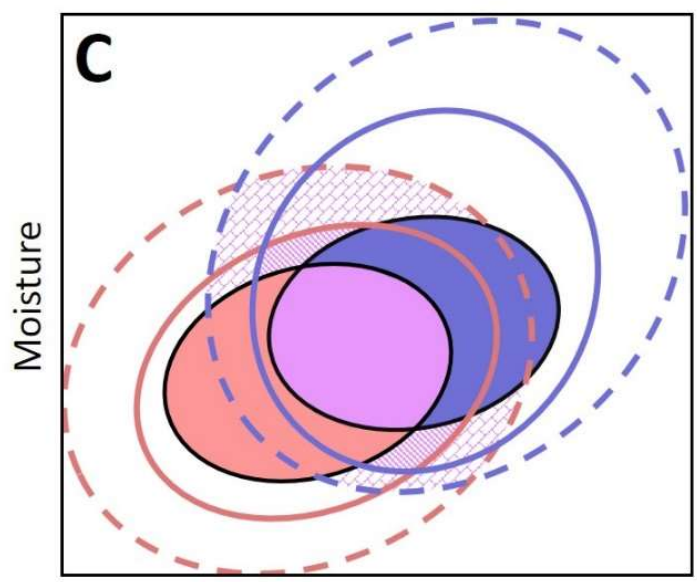

Temperature

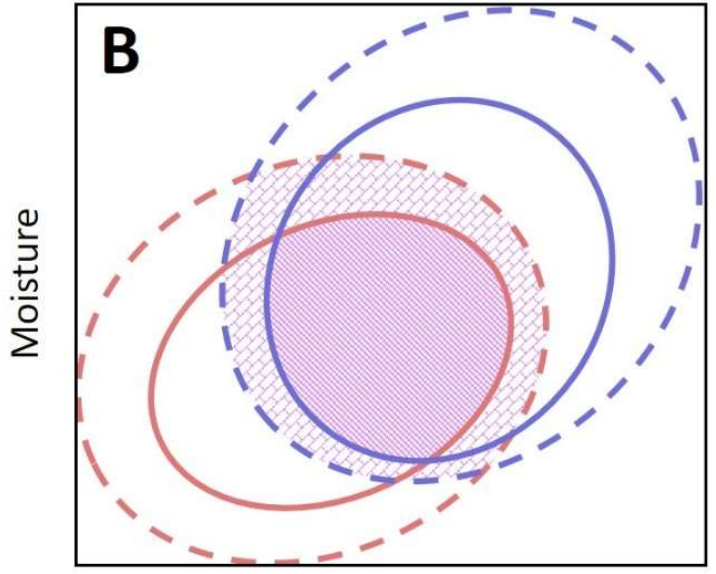

Temperature

Legend

$\square$ Niche expansion

Niche unfilling

Niche conserved

Analog climate (75\%)

Analog climate (99\%)

--. Introduced climate space (99\%)

— Introduced climate space (75\%)

--. Native climate space (99\%)

- Native climate space $(75 \%)$

Figure 10. Categories of climate niche space. A) Species occurrences in the native and introduced ranges; $\mathbf{B}$ ) background occurrence densities of the native and introduced ranges at thresholds of $75^{\text {th }}$ and $99^{\text {th }}$ percentile of marginal climate space, indicating analog and non-climate space; C) The combined overlap of species occurrence densities and background occurrence densities, so that niche expansion and total niche change could be calculated in analog and nonanalog climates to include the $75^{\text {th }}-99^{\text {th }}$ percentiles of available climate space. 


\subsubsection{Predicting trait change through time from niche shifts}

\subsubsection{Quantifying trait change through time:}

The rate of trait change through time was quantified for each species trait using a general least squares analysis (GLS), also known as spatial regression, using the nlme package in $R$ v. 3.2.1 ( $R$ Core Team 2013). Each model explained variation in herbarium trait values for a species as a log-linear relationship, with main effect terms for year, temperature and moisture. Each trait measurement was $\log _{10}$ transformed, both to satisfy assumptions of data normality and so that the proportional change in a trait through time could be compared across different species. The coordinates of each herbarium specimen were included in each model; this allowed for the calculation of a variogram that modelled spatial dependency between data points as a function of their proximity to each other, based on a Gaussian correlation structure.

$$
\text { Trait } \sim \text { Year }+ \text { Temperature }+ \text { Moisture }+[1 \mid \text { Coordinates }]
$$

(Equation 3.)

Climate terms were included in the model as covariates, in order to predict the rate of trait change through time, independent of trait variation across a climatic gradient. The geo-referenced locations of each herbarium specimen were included as a random term to account for any potential spatial autocorrelation in trait values.

The beta coefficient of the year term gives the direction and proportional rate of trait change through time for each trait-species combination, while accounting for the effects of climate. Interaction terms were not included so that there would only be a single slope value for rate of trait change through time, which could then be related to niche shift. The beta coefficient $(B)$ of the year term for each trait-species combination was used as the effect size in the subsequent stages of the analysis (Tables S10 -S11). 


\subsubsection{Question 1: Predicting the rate of trait change through time from the magnitude of a climate niche shift}

A meta-analysis approach was used to combine the rate of trait change through time for each species into an overall multi-species effect size for each trait. The beta coefficient and standard error for the year term in the GLS analysis were used to give an effect size and variance in a meta-analysis random-effects model. Beta-coefficients are not often used as effect sizes in metaanalyses, due to disparity in their measurement between studies. However, it is appropriate to synthesise beta-coefficients in this manner if the following assumptions are met: i) the response variable is measured in the same way across groups, ii) the predictor variable is measured in the same way across groups, and iii) additional predictors variables are measured in the same way across groups (Becker \& Wu 2007). Using this approach, the contribution of each species to the overall effect size was weighted as the inverse of that species' variance plus the between-species variance in each range. As the aim of question one was to make an assessment of absolute rate of change through time, the direction of the trait change was not included. Consequently negative beta-coefficients (i.e., decrease through time) were converted to positive values. Summary effects were assessed separately for introductions into New Zealand and Australia. To explore how the rate of trait change through time varied in response to the magnitude of climatic niche shifts, a mixed effects meta-analysis model (meta-regression) was performed. The degree of niche overlap (Schoener's D), niche expansion and total niche change (calculated at levels of $75^{\text {th }}-99 t^{\text {th }}$ percentile of marginal climate space) were introduced as predictor variables in separate analyses. 


\subsubsection{Question 2: Predicting the direction and rate of trait change through time from the magnitude and direction of niche centroid shift}

Question two set to ask if the magnitude and direction of climate niche shift could predict the rate and direction of trait change through time. The shift in niche centroid between the native and introduced range was used to address this question. This approach describes the niche shift as a vector in climate space, and thus the direction can be accounted for. The niche centroid for each range was calculated as the weighted mean value of the species occurrence densities in climate space. The magnitude of the niche centroid shift is the difference between the native range centroid and the introduced range centroid. Niche centroid shifts were calculated along a 1-dimensional axis for each climate variable (temperature and moisture), and along a normalised 2-dimensional axis for both climate variables (Figure 11). The normalised 2-dimensional axis accounts for the total niche centroid shift in shared climate space. To calculate this, each axis was re-scaled to range from one to 100 so that both climate variables were in comparable units. Then distance of the hypotenuse between two niche centroids was calculated using Pythagoras' theorem.

The purpose of this analysis was to see if the direction and magnitude of a climate niche shift predicted the magnitude and direction of trait change through time that would be expected based on general trait-climate relationships taken from the literature. For example, following a shift into a drier climate we may expect for trait change through time towards a decline in height and a decrease in leaf area and shape (Fonseca et al. 2000; McDonald et al. 2003; Moles et al. 2011). In niche shift scenarios that would be expected to drive an increase in a specific trait through time, the niche centroid shift was given a positive value (corresponding with a trait increase through time if correct). In scenarios in which a decline in specific trait would be predicted, the niche centroid shift was given a negative value (corresponding with a trait decrease through time if correct; Figure 12). For a summary of predictions, see Table 8 . The niche centroid shift metrics 
(and their directions) were then included separately as predictors in a mixed-effects metaanalysis. Following this, all positive beta-coefficients for predictors are in accordance with predictions, while all negative beta-coefficients indicate contrary predictions.

Table 8. The predicted direction of trait change in response to the direction of the niche centroid shift.

\begin{tabular}{|c|c|c|}
\hline Trait & $\begin{array}{l}\text { Predicted direction of change in response } \\
\text { to niche shift }\end{array}$ & Source \\
\hline Height & $\begin{array}{l}\text { Increase with shift into wetter niche } \\
\text { Decrease with shift into drier niche } \\
\text { Increase with shift into hotter niche } \\
\text { Decrease with shift into colder niche }\end{array}$ & $\begin{array}{l}\text { Baher et al. (2002); } \\
\text { Walker et al. (2006); } \\
\text { Moles et al. (2009; 2014) }\end{array}$ \\
\hline Leaf Shape & $\begin{array}{l}\text { Increase (Narrower) with shift into hotter } \\
\text { and/or drier niche } \\
\text { Decrease (Wider) with shift into colder and } \\
\text { wetter niche }\end{array}$ & $\begin{array}{l}\text { Yates et al. (2010); } \\
\text { Peppe et al. (2011); } \\
\text { Guerin et al. (2012); }\end{array}$ \\
\hline Leaf area & $\begin{array}{l}\text { Decrease with shift into drier niche } \\
\text { Increase with shift into hotter niche } \\
\text { Increase with shift into wetter niche }\end{array}$ & $\begin{array}{l}\text { Parkhurst \& Loucks (1972); } \\
\text { Fonseca et al. (2000); } \\
\text { Khan et al. 2001; } \\
\text { McDonald et al. (2003); } \\
\text { Xu et al. (2009); } \\
\text { Yates et al. 2010; } \\
\text { Peppe et al. (2011); } \\
\text { Moles et al. (2014) }\end{array}$ \\
\hline
\end{tabular}




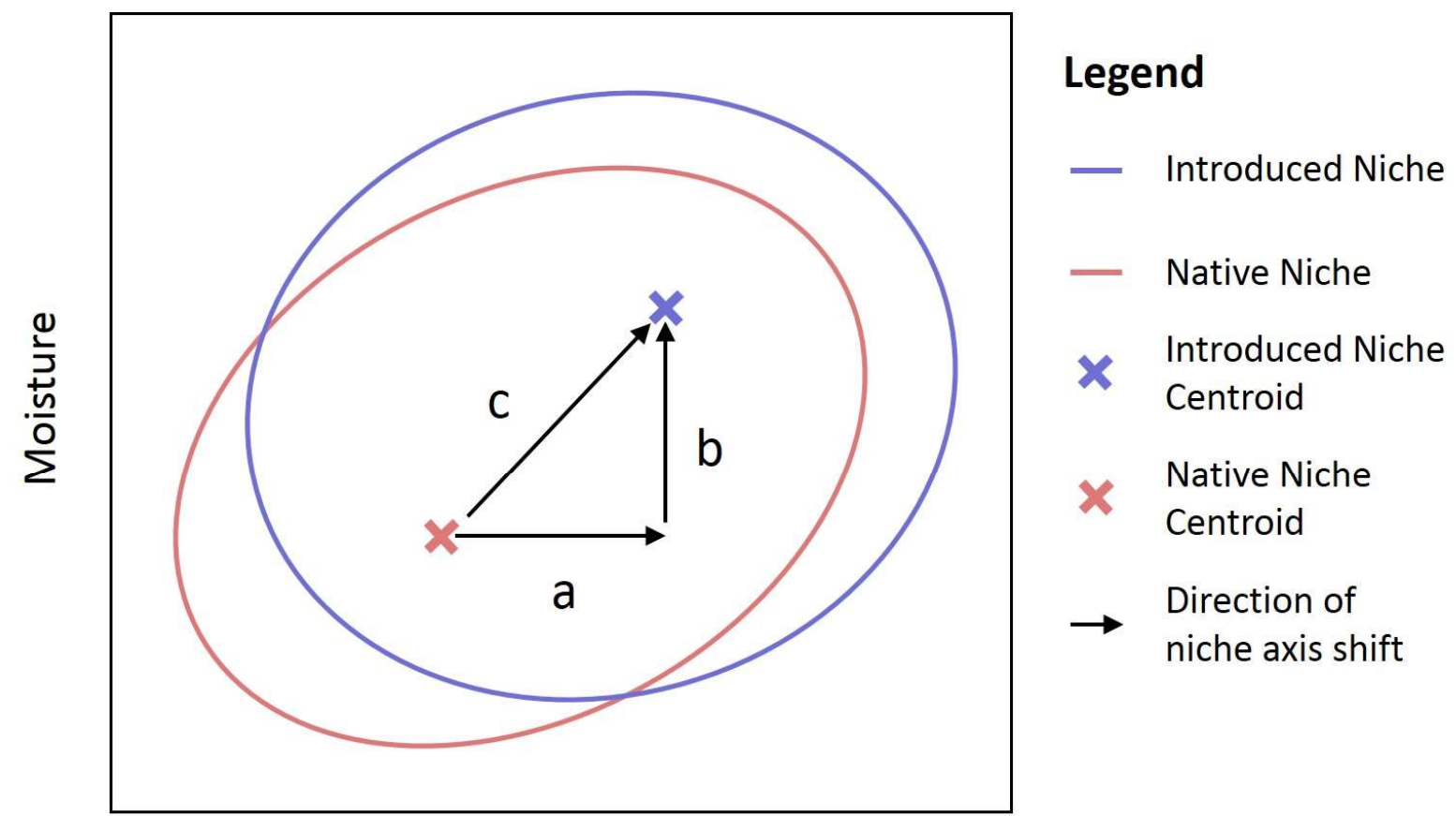

Temperature

Figure 11. The calculation of a niche centroid shift in climate space. Niche centroids $(\mathbf{x})$ were calculated as the weighted mean species occurrence densities for each niche. Niche centroid shifts were calculated as the difference between each centroid along a respective axis in climate space: a) Niche centroid shift along temperature axis; b) Niche centroid shift along moisture axis; c) Niche centroid shift along a normalized 2- dimensional axis (calculated from Pythagoras' theorem: $c^{2}=a^{2}+b^{2}$ ). 


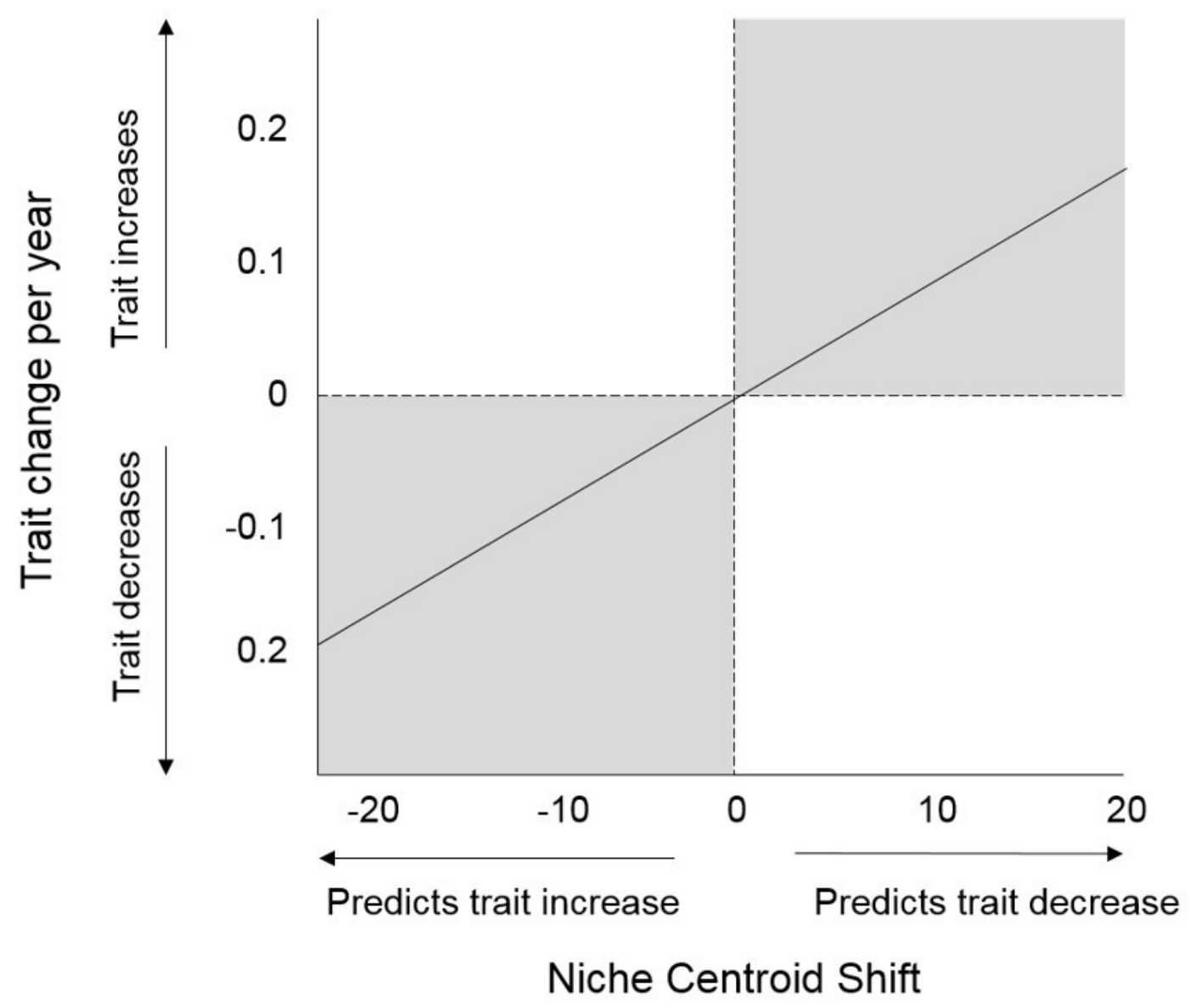

Figure 12. Example plot of how the magnitude and direction of a niche centroid shift would be expected to predict the rate and direction of change of a given trait through time. Grey areas indicate sections of the graph in which the direction of the niche centroid shifts correctly predicts the direction of the trait change through time, while white areas indicate incorrect predictions. The black line represents an example regression slope, in which trait change through time is correctly predicted by the niche centroid shift. 


\subsubsection{Question 3: Trait change through time in novel climate space}

The third question asks whether trait change through time occurs at a faster rate in herbarium specimens from areas of novel climate space. The GLS analysis was repeated for herbarium specimens that were subsampled to only include individuals that occurred in: i) novel climate space (niche expansion) and ii), shared climate space (niche conserved). A mixed effects metaanalysis model was used to test if the rate of trait change through time was significantly greater in novel climate space. Hedges' D is a commonly used effect size in meta-analysis, calculated as the difference between two means. Here, the difference in the rate of change through time (i.e., the beta-coefficient) between novel and shared climate space was used as an effect size $\left(B_{\text {diff }}\right)$. This was calculated by subtracting the beta-coefficient for the trait change through time in the novel climate $\left(B_{n}\right)$ space from the beta-coefficient if the shared $\left(B_{s}\right)$ climate space $\left(B_{\text {diff }}=B_{n}-B_{s}\right)$. The variance $\left(V_{B}\right)$ of the difference between two uncorrelated effect sizes is calculated simply as the sum of their variances (Borenstein et al. 2009a). Positive values indicate a faster rate of trait change through time in novel climate space, while negative values indicate a faster rate of change through time in shared climate space. Introduced species that did not have a suitable minimum number of herbarium samples in both novel and shared climate space $(n>20)$, were removed from this analysis. As so few species introduced to Australia had a sufficient number of samples occurring in novel climate space, this analysis was only performed on a subset of the New Zealand species. If a herbarium specimen fell outside of a niche category in climate space (i.e., was outside niche expansion or niche conserved), then it was extrapolated to be included in the niche expansion category. For an example, see Figure 9 in which some herbarium specimens fall outside of the predicted climate niche. 


\subsection{Results}

\subsubsection{Herbarium trait change through time}

A meta-analysis approach using a random effects model found the overall proportional rate of trait change through time over 100 years (including direction) to not be significantly different from zero for height $(\mathrm{AU}=1.07[95 \% \mathrm{Cl}: 0.93$ to 0.123$] ; \mathrm{NZ}=0.88$ [0.76 to 1.01]), leaf area $(\mathrm{AU}=0.94$ [0.73 to 1.22$] ; \mathrm{NZ}=0.92$ [0.68 to 0.25$])$ and leaf shape $(\mathrm{AU}=0.97$ [0.88 to 1.07$] ; \mathrm{NZ}=1.00$ [0.93 to 1.07]). See Figure 13 for a summary of the change of time for each species and the overall rate of change for each trait.

\subsubsection{Summary of key niche differences}

Species introductions to New Zealand underwent climate niche shifts of a greater magnitude than those in Australia, for the degree of niche overlap (Schoener's D), Niche Expansion and Total Niche Change in both analog and non-analog climates (Figure 14). Niche shifts were greater for all metrics when calculated to also include non-analog climate space. When niche shifts were assessed including non-analog climate space, the percentile of marginal climate space had little effect on niche expansion or total niche change metrics. When assessed using only analog (shared) climate space, the percentile of marginal climate space showed more variation. In this case, niche expansion and total niche change were significantly reduced when a lower marginal climate percentile (i.e., more conservative niche envelope) was used for New Zealand, but not for Australia. See tables S9 - S10 for a summary of niche shift metrics calculated for each species. 


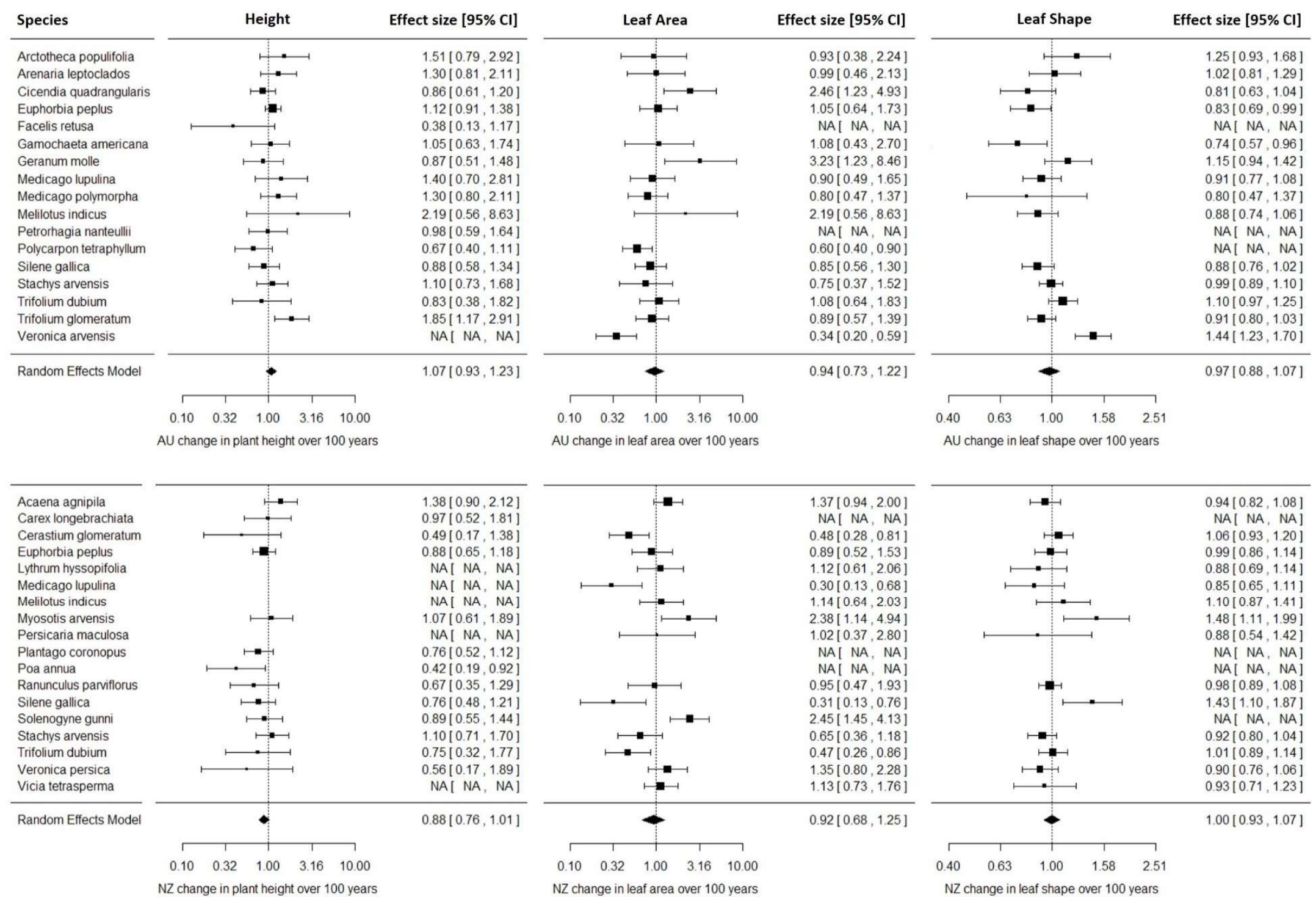

Figure 13. The proportional rate and direction of trait change through time for each species introduced to Australia (top) and New Zealand (bottom). Diamonds represent the overall rate of change for that trait, calculated from a random effects model meta-analysis. 


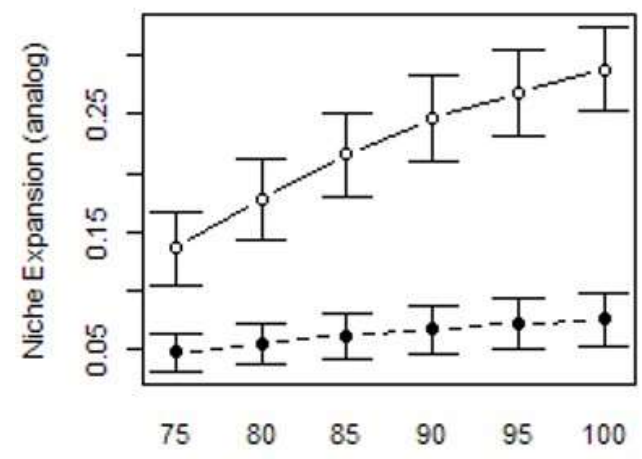

Percentile of marginal climate included

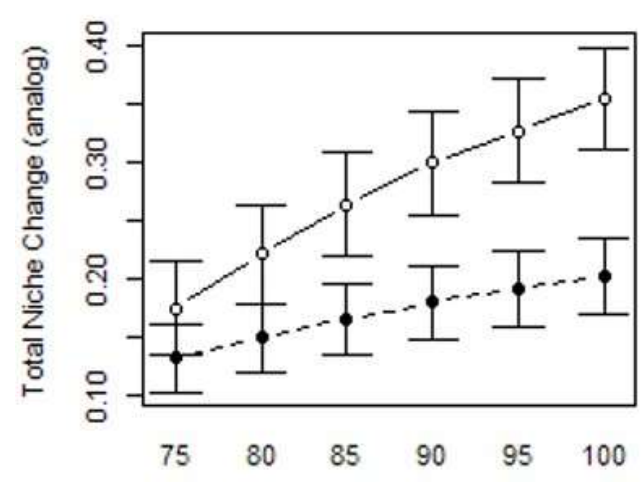

Percentile of marginal climate included

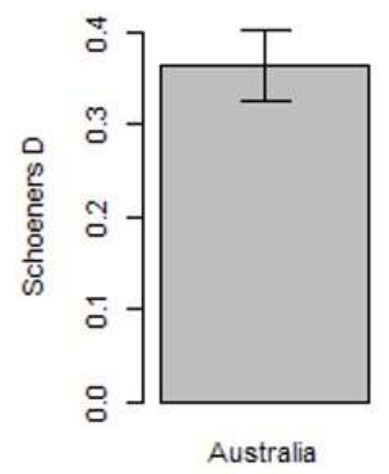

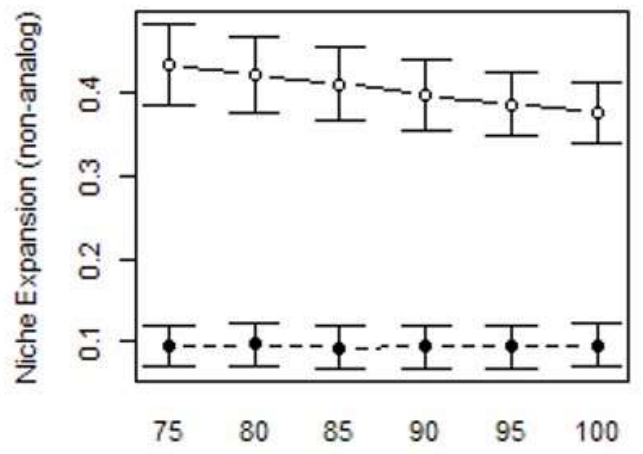

Percentile of marginal climate included

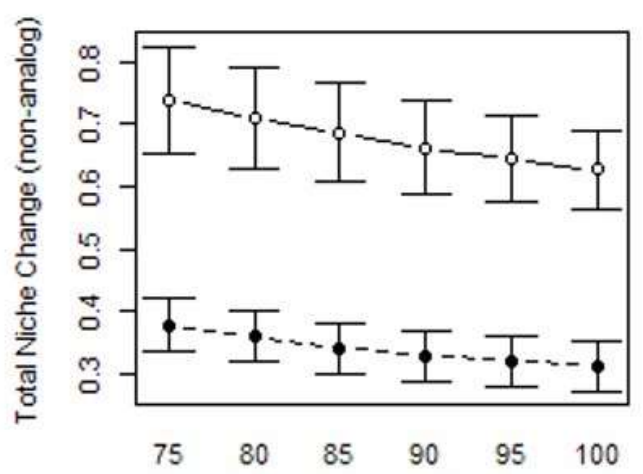

Percentile of marginal climate included

Introduced range

Figure 14. Summary of mean niche shift metrics for Australia (Black) and New Zealand (White) calculated to include different percentiles of marginal climate space $\left(75^{\text {th }}\right.$ to $99^{\text {th }}$ percentile). 


\subsubsection{Does the magnitude of the niche shift predict the rate of trait change through time?}

The absolute rate of trait change through time for herbarium specimens was poorly predicted by the magnitude of the niche shift, for all niche shift metrics examined (Table 9). There was little difference in the predictions made when climate thresholds were calculated at intervals between the $75^{\text {th }}$ and $99^{\text {th }}$ percentile. Consequently, I present results for climate niches calculated at the $99^{\text {th }}$ percentile of available climate space, as this gave the more conservative estimate of a species niche shift. Nearly all relationships between the rate of trait change and the magnitude of niche shift were non-significant $(p>0.05)$. However, the rate of leaf area change through time in New Zealand was found to decrease with increasing magnitude of niche shift for total niche change $(B=-0.544, p=0.009)$ and niche expansion $(B=-0.605, p=0.019)$ when assessed using only analog climates. This negative relationship was counter to my expectations.

\subsubsection{Does the magnitude and direction of niche centroid shift predict the direction and rate of trait change through time?}

The rate and direction of plant trait change through time was poorly predicted by the direction and magnitude of the niche centroid shift into the introduced range. This was the case for change in plant height, leaf area and leaf shape when assessed along a single axis of temperature or moisture, and along a normalised two-dimensional axis of the two variables combined (Table 10). All of these tests were non-significant, apart from leaf shape change through time which showed a negative relationship with the direction predicted from the niche centroid shift in Australia $(B=-$ $0.002, p=0.04)$. This negative relationship indicates that the direction of leaf shape change through time was in the opposite direction to what was predicted from the direction of the niche centroid shift in climate space. 
Table 9. The proportional rate of trait change through time in response to the magnitude of the climate niche shift. $B$ is the raw beta-coefficient for the change in the rate of trait change through time over 100 years per unit niche shift. Negative values represent a decrease in the rate of trait change through time, while positive values represent an increase. $10^{(B)}$ is the exponent transformation of the beta-coefficient, representing the change in the proportional rate of trait change through time over 100 years per unit niche shift. $10^{(B)}$ values are centred on 1 ; values $>1$ indicate an increase in the rate of trait change through time per unit niche shift, while values $<1$ indicate a decrease. Climate thresholds were calculated at the $99^{\text {th }}$ percentile of the available climate space.

\begin{tabular}{|c|c|c|c|c|c|c|}
\hline Range & Trait & Niche Shift Metric & Climate threshold & $B$ & $10^{(B)}$ & $p$ \\
\hline \multirow{15}{*}{ Australia } & \multirow{5}{*}{ Height } & \multirow{2}{*}{$\begin{array}{l}\text { Total Niche } \\
\text { Change }\end{array}$} & Analog climate & -0.185 & 0.65 & 0.346 \\
\hline & & & Non-analog climate & 0.009 & 1.02 & 0.943 \\
\hline & & \multirow{2}{*}{ Niche Expansion } & Analog climate & -0.091 & 0.81 & 0.648 \\
\hline & & & Non-analog climate & -0.027 & 0.93 & 0.875 \\
\hline & & Schoener's D & & -0.033 & 0.93 & 0.938 \\
\hline & \multirow{5}{*}{$\begin{array}{l}\text { Leaf } \\
\text { Area }\end{array}$} & \multirow{2}{*}{$\begin{array}{l}\text { Total Niche } \\
\text { Change }\end{array}$} & Analog climate & -0.544 & 0.29 & 0.011 \\
\hline & & & Non-analog climate & -0.229 & 0.59 & 0.178 \\
\hline & & \multirow{2}{*}{ Niche Expansion } & Analog climate & -0.605 & 0.25 & 0.020 \\
\hline & & & Non-analog climate & -0.251 & 0.56 & 0.336 \\
\hline & & Schoener's D & & -0.459 & 0.35 & 0.416 \\
\hline & \multirow{5}{*}{$\begin{array}{l}\text { Leaf } \\
\text { Shape }\end{array}$} & \multirow{2}{*}{$\begin{array}{l}\text { Total Niche } \\
\text { Change }\end{array}$} & Analog climate & -0.074 & 0.85 & 0.322 \\
\hline & & & Non-analog climate & -0.013 & 0.98 & 0.742 \\
\hline & & \multirow{2}{*}{ Niche Expansion } & Analog climate & -0.084 & 0.83 & 0.311 \\
\hline & & & Non-analog climate & -0.044 & 0.91 & 0.454 \\
\hline & & Schoener's D & & -0.117 & 0.76 & 0.366 \\
\hline \multirow{15}{*}{$\begin{array}{l}\text { New } \\
\text { Zealand }\end{array}$} & \multirow{5}{*}{ Height } & \multirow{2}{*}{$\begin{array}{l}\text { Total Niche } \\
\text { Change }\end{array}$} & Analog climate & 0.016 & 0.68 & 0.321 \\
\hline & & & Non-analog climate & 0.001 & 0.74 & 0.379 \\
\hline & & \multirow{2}{*}{ Niche Expansion } & Analog climate & 0.102 & 0.49 & 0.234 \\
\hline & & & Non-analog climate & 0.161 & 0.62 & 0.374 \\
\hline & & Schoener's D & & 0.146 & 1.62 & 0.176 \\
\hline & \multirow{5}{*}{$\begin{array}{l}\text { Leaf } \\
\text { Area }\end{array}$} & \multirow{2}{*}{$\begin{array}{l}\text { Total Niche } \\
\text { Change }\end{array}$} & Analog climate & 0.278 & 2.14 & 0.294 \\
\hline & & & Non-analog climate & 0.132 & 1.48 & 0.538 \\
\hline & & \multirow{2}{*}{ Niche Expansion } & Analog climate & 0.114 & 1.62 & 0.678 \\
\hline & & & Non-analog climate & -0.017 & 1.12 & 0.903 \\
\hline & & Schoener's D & & 0.049 & 1.07 & 0.896 \\
\hline & \multirow{5}{*}{$\begin{array}{l}\text { Leaf } \\
\text { Shape }\end{array}$} & \multirow{2}{*}{$\begin{array}{l}\text { Total Niche } \\
\text { Change }\end{array}$} & Analog climate & -0.076 & 0.83 & 0.391 \\
\hline & & & Non-analog climate & -0.029 & 0.93 & 0.733 \\
\hline & & \multirow{2}{*}{ Niche Expansion } & Analog climate & -0.140 & 0.69 & 0.141 \\
\hline & & & Non-analog climate & -0.113 & 0.74 & 0.184 \\
\hline & & Schoener's D & & 0.012 & 1.02 & 0.937 \\
\hline
\end{tabular}


Table 10. The relationship between the magnitude and direction of the niche centroid shift and the direction and rate of trait change through time. Niche centroid shifts were assessed along a single axis for each climate variable and a combined two-dimensional shift across both normalised axes. Niche centroid shift metrics were assessed independently, as predictors in a mixed effects meta-analysis. The direction of the 2-D niche centroid shift was interpreted as: positive betacoefficients indicate that the direction of the niche centroid shift correctly predicted the direction of the trait change through time, while negative beta-coefficients indicate counter predictions. $10^{(B)}$ is the exponent transformation of the beta-coefficient, representing the change in the proportional rate of trait change through time over 100 years per unit niche centroid shift. $n=$ number of species included in the analysis. Climate thresholds were calculated at the $99^{\text {th }}$ percentile of the available climate space.

\begin{tabular}{|c|c|c|c|c|c|c|}
\hline Range & Trait & $n$ & Niche axis shift & $B$ & $10^{(B)}$ & $p$ \\
\hline \multirow{9}{*}{ Australia } & \multirow{3}{*}{ Height } & \multirow{3}{*}{16} & Moisture index of the driest quarter & 0.001 & 0.62 & 0.878 \\
\hline & & & Mean temperature of the warmest quarter & -0.007 & 1.05 & 0.746 \\
\hline & & & 2-D Centroid shift & 0.001 & 0.95 & 0.547 \\
\hline & \multirow{3}{*}{$\begin{array}{l}\text { Leaf } \\
\text { Area }\end{array}$} & \multirow{3}{*}{15} & Moisture index of the driest quarter & 0.001 & 2.09 & 0.842 \\
\hline & & & Mean temperature of the warmest quarter & -0.005 & 0.95 & 0.818 \\
\hline & & & 2-D Centroid shift & 0.002 & 1.55 & 0.791 \\
\hline & \multirow{3}{*}{$\begin{array}{l}\text { Leaf } \\
\text { Shape }\end{array}$} & \multirow{3}{*}{15} & Moisture index of the driest quarter & -0.001 & 0.59 & 0.513 \\
\hline & & & Mean temperature of the warmest quarter & 0.007 & 1.02 & 0.586 \\
\hline & & & 2-D Centroid shift & -0.002 & 0.60 & 0.040 \\
\hline \multirow{9}{*}{$\begin{array}{l}\text { New } \\
\text { Zealand }\end{array}$} & \multirow{3}{*}{ Height } & \multirow{3}{*}{12} & Moisture index of the driest quarter & -0.132 & 0.74 & 0.795 \\
\hline & & & Mean temperature of the warmest quarter & -0.018 & 0.96 & 0.534 \\
\hline & & & 2-D Centroid shift & 0.003 & 1.86 & 0.704 \\
\hline & \multirow{3}{*}{$\begin{array}{l}\text { Leaf } \\
\text { Area }\end{array}$} & \multirow{3}{*}{14} & Moisture index of the driest quarter & 0.551 & 3.55 & 0.517 \\
\hline & & & Mean temperature of the warmest quarter & -0.015 & 0.97 & 0.818 \\
\hline & & & 2-D Centroid shift & 0.012 & 14.13 & 0.382 \\
\hline & \multirow{3}{*}{$\begin{array}{l}\text { Leaf } \\
\text { Shape }\end{array}$} & \multirow{3}{*}{13} & Moisture index of the driest quarter & 0.112 & 1.35 & 0.512 \\
\hline & & & Mean temperature of the warmest quarter & -0.011 & 0.98 & 0.342 \\
\hline & & & 2-D Centroid shift & 0.001 & 1.35 & 0.676 \\
\hline
\end{tabular}


4.3.5 Do herbarium specimens sampled from areas of climate space change through time at a faster rate?

New Zealand herbarium specimens sampled from novel climate space did change through time at a slightly faster rate than those sampled from climate space shared with the native range (Figure 15), however this difference was not significant for height $\left(B_{\text {diff }}=0.06[95 \% \mathrm{Cl}:-0.07\right.$ to $0.19])$, leaf area $\left(B_{\text {diff }}=0.09[95 \% \mathrm{Cl}:-0.42\right.$ to 0.6$\left.]\right)$ or leaf shape $\left(B_{\text {diff }}=0.04[95 \% \mathrm{Cl}:-0.01\right.$ to 0.09]). As there were not enough herbarium samples from novel climate space in Australia, this analysis could not be performed for introductions to the Australian range. 


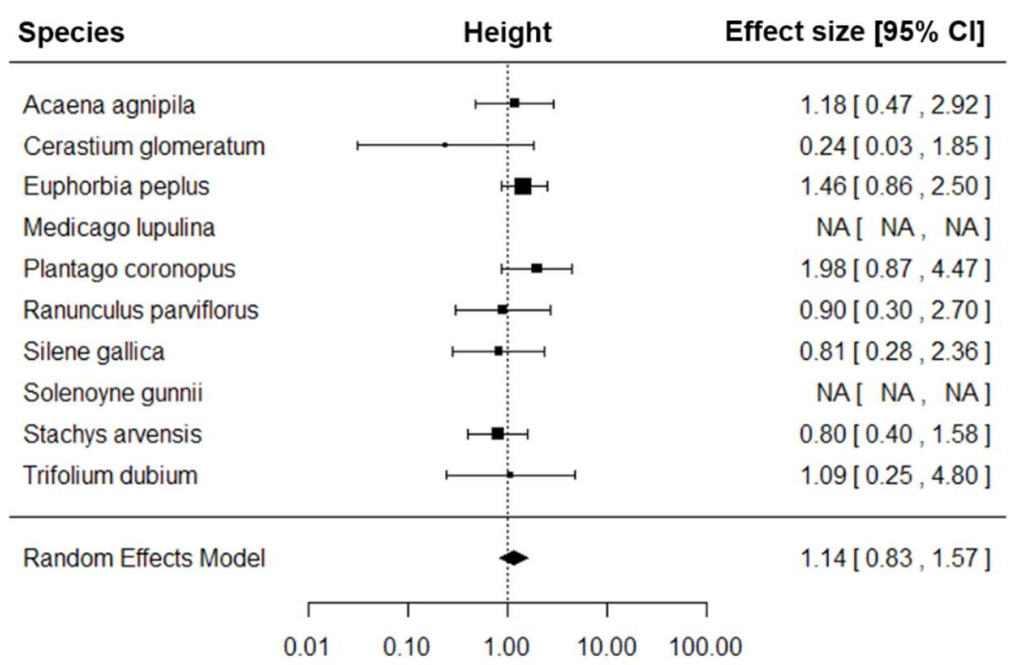

Species

Leaf Area

Effect size $[95 \% \mathrm{Cl}]$

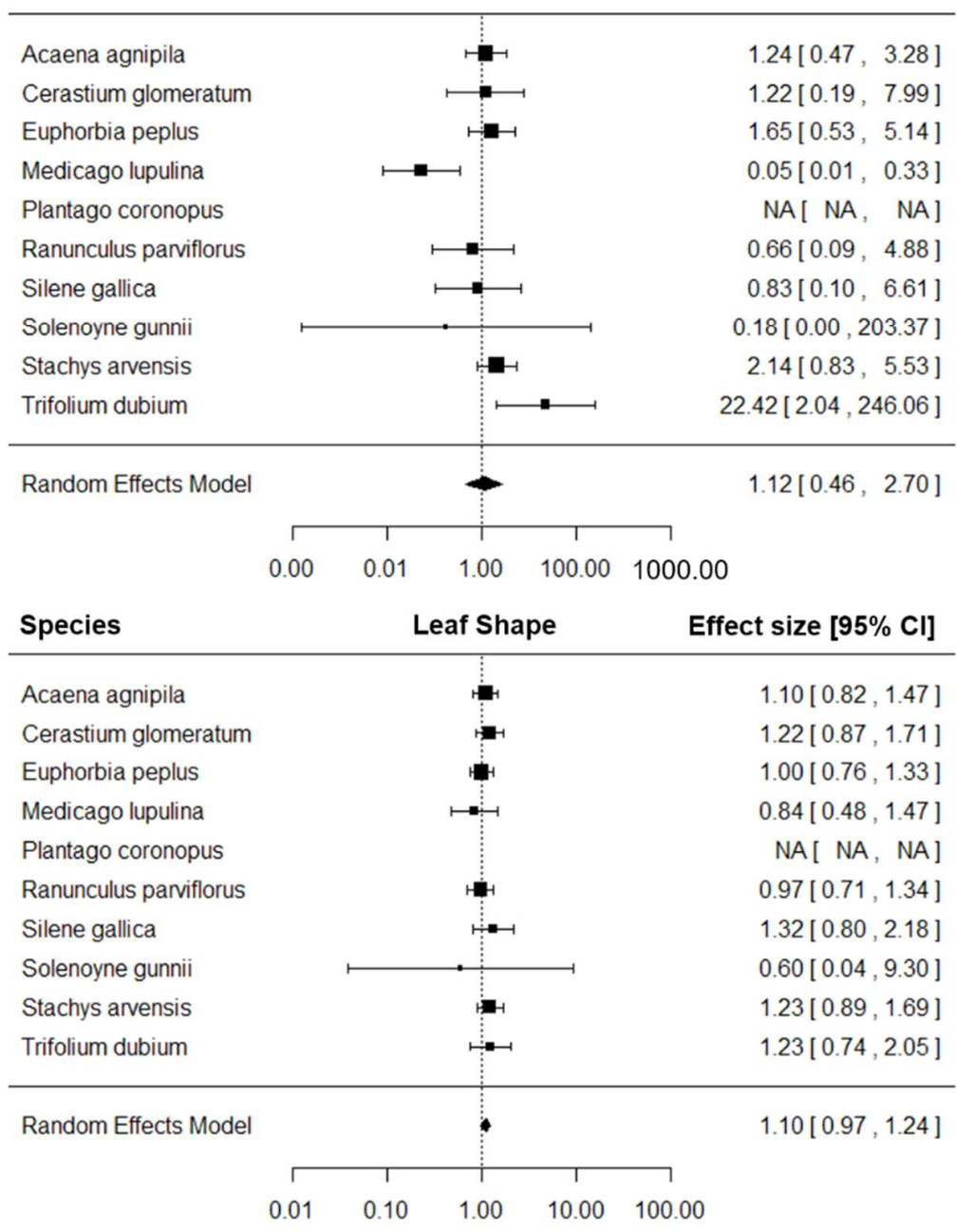

Proportional difference in the rate of trait change over 100 years

Shared climate faster Novel climate faster
Figure 15. The difference in the proportional rate of trait change through time for New Zealand herbarium specimens sampled from novel climate space versus those sampled from climate space shared with the native range, over a 100 year period. The difference in beta-coefficients between ranges ( $\left.B_{\text {diff }}\right)$ has been back transformed (as $10^{(B \times 100}$ ) to represent a proportional difference in the rate trait of change through time. Values $>1$ indicate that the rate of trait change through time was faster in novel climate space, values $<1$ indicate that the rate of change was faster in shared climate space. 


\subsection{Discussion}

In this study I used a range of techniques to quantify the magnitude and direction of realised climate niche shifts. None of these methods were very effective in predicting morphological trait change through time across a suite of species. Although some species did show substantial climatic niche shifts, this did not correlate with the rate of trait change through time. While niche shifts have been previously documented in introduced species (Broennimann et al. 2007; Gallagher et al. 2010; Tingley et al. 2014), this is the first study to combine evidence of niche shifts in multiple species with their trait responses in the introduced range. A shift in climate following introduction to a new range is thought to have driven rapid evolution of morphological traits in Tamarix ramosissima (Sexton et al. 2002) and Hypericum perforatum (Maron et al. 2004) in North America. Felker-quinn et al. (2013) conducted a meta-analysis, indicating that introduced plants showed divergent traits from their native conspecifics, however there was no clear pattern to these trait differences nor were they associated with an increase in fitness in the introduced range.

\subsubsection{Niche shifts did not predict trait change}

Despite seeing evidence for both niche shifts and trait change through time in introduced herbarium plant specimens, the two seemed relatively un-coupled in interspecific analyses. The absolute rate of trait change through time was poorly predicted by the magnitude of the climate niche shift into the introduced range, with only two out of 30 tests showing a significant relationship. Additionally, the relationship between trait change through time and these two niche shift indices was in the opposite direction to what was expected. With leaf area showing a slower absolute rate of change through time in species introduced to New Zealand that had undergone a greater niche expansion or total niche change. 
When incorporating the direction of the niche shift (through assessing niche centroids) and direction of trait change through time into the analysis, I found similarly poor predictions. The one significant effect detected was also in the opposite direction to what was expected: the rate of leaf shape change through time had a negative relationship with the magnitude of niche centroid shift, in species introduced to Australia. As climate was hypothesised to be a key driver of trait change in plants when shifting into a new climate niche, I next considered that trait change through time may be more likely to occur in areas of novel climate space. However, a comparison of New Zealand herbarium specimens sampled from areas of novel and conserved climate space revealed no difference in their absolute rate of trait change through time.

\subsubsection{Possible reasons why niche shifts did not predict trait change through time}

If climate is not a key driver of trait change through time of species in the introduced range, then it follows that the rate of change would be poorly predicted by climate niche shifts. This is the most straightforward explanation for the results found in the study. However, there a number of other factors that could also contribute to this, which I will now outline.

\subsubsection{Other traits may be selected for instead}

Firstly, it could be that the specific climate variables selected for in this study did not have a strong influence in driving trait change for some of these species. The climate variables were selected to cover two key variables that could be expected to affect plant growth: limiting water supply and temperature. These variables are known to have an influence on morphological trait variation at the global scale (Moles et al. 2009; Moles et al. 2011). It is possible that these climate variables could be having an influence on plant growth and selective pressures in the introduced range, but not on the morphological traits measured in this study. A change in climate (temperature and/or water availability) could drive a response in many other plant traits, including but not limited to; 
stomatal size and density (Xu \& Zhou 2008), plant water-use efficiency (Medrano et al. 2011; Linares \& Camarero 2011), total plant biomass (Hobbie \& Chappin III 1998), and biomass allocation to roots (Poorter et al. 2009). It is possible that some species could have responded to a change in climate in the introduced range by adapting these traits, reducing the selective pressure acting on the traits measured in this study.

\subsubsection{Unaccounted for abiotic or biotic factors}

It should also be considered that climate variables do not cover all axes of the multidimensional Hutchinsonian niche, and changes in other abiotic (e.g., soil nutrients, disturbance) and biotic factors (e.g., herbivory, competition) could represent niche shifts in the introduced range (Holt 2009). Biotic factors are likely to have an influence in driving trait change in some species. The escape from natural enemies and EICA hypotheses suggest that plants may be expected to undergo an increase in size in the introduced range (Blossey and Notzhold 1995; Keene \& Crawley 2002). It is possible that biotic interactions may simultaneously act with abiotic factors to put selective pressure on ecologically important traits in the same or in opposing directions (Felker-Quinn et al. 2013). For example, a species that has undergone a large niche shift into a drier climate may select for a decrease in height in response to water availability, while simultaneously selecting for an increase in height due to escaping natural enemies from the native range. This could result in no perceivable change in plant height through time, despite undergoing a relatively large niche shift.

\subsubsection{Mismatch in scale}

Another important aspect to consider are the spatial scales used to assess the rate of trait change through time, climate niche shifts, and potential mismatches between them. Niche shifts were assessed at a regional level, giving a general view of the entire climate space occupied in the native and introduced ranges. A potential issue with this, especially for species with large native 
ranges, is that the location of the source population is often unknown. Thus, the shift in climate space that an introduced population has actually undergone may be masked by the extent of the rest of the native range niche. This broad scale assessment of the niche may also be at a mismatch when attempting to compare it to patterns in herbarium specimens that may experience other local sources of variation. Trait change through time was assessed at the country level, while controlling for location and climatic factors that may cause spatial non-independence or variation in trait values. However, phenotypic plasticity in response to other local scale factors, or the method of sampling of herbarium specimens, may also lead to variation in trait values that could mask the overall patterns in trait change through time.

\subsubsection{Phenotypic plasticity}

Phenotypic plasticity is a way in which non-native species can better cope with a change in environmental conditions when introduced to a new range (Sexton 2002; Richards et al. 2006). A capacity for greater plasticity may also be rapidly selected for following introduction to a new range, allowing individuals to respond to a change in environmental conditions through shifting their traits to a more optimum value (Thompson 1991; Gomez-mestre et al. 2013; Lande 2015). Kolbe et al. (2012) documented a rapid increase in thermal plasticity in the lizard Anolis cristatellus, following a climatic niche shift to lower minimum temperatures in the introduced range. This increase in plasticity can enable individuals in a population to shift their traits towards a new optimum phenotype, on which genotypes will then be selected upon over time (Gomezmestre et al. 2013; Lande 2015). Consequently, phenotypic plasticity to local scale environmental variation could mask trait change through time towards a new optimum phenotype. 


\subsubsection{Varying rate of trait change}

The rate of trait change through time for many species traits may not occur on a log-linear scale, as predicted in this study. Low population densities and/or genetic diversity shortly after colonisation are thought to contribute to lag phases and slower rates of adaptation (Mooney \& Cleland 2001; Sakai et al. 2001; Holt et al. 2005; Suarez \& Tsutsui 2008). Alternatively, it has been suggested that the sudden change in the environment when introduced to a new range can potentially result in stronger selection pressures and more rapid rates of trait change early on during establishment. (Thompson 1998; Reznick and Ghalambor 2001; Lambrinos 2004). Floresmoreno et al. (2015) used herbarium specimens to examine how variable trait change through time was over $\sim 200$ year period, for three plant species introduced to the United Kingdom. They found that none of these species showed evidence for a lag phase in their trait change through time over the first 100 years, but did continually show varying rates of trait change throughout the 200 year period they were assessed across.

\subsubsection{Variation between species}

The rate of trait change through time may be more specific to the properties of each individual species, rather than directly related to the magnitude of niche shift which it has undergone. This may explain why some species have undergone relatively large niche shifts, but then show very low rates of trait change through time. This could occur if a species changes in its environmental tolerance rather than changing its trait values, when introduced to a novel environment (Hulme \& Barret 2013). Conversely some species may undergo a small niche shift, but show a large change in certain traits that have been driven by unaccounted for abiotic or biotic factors (Keane \& Crawley 2002; Felker-Quinn et al. 2012; Hulme \& Barret 2013), or vice versa.

Each of the factors outlined above may contribute to additional noise and variability in: i) the prediction of trait change through time, ii) quantifying climate niche shifts, and iii) combining the 
two approaches, to predict trait change through time in relation to climate niche shifts. While some degree of noise and uncertainty can be an issue associated with predictions of trait change from herbarium specimens (Miller-Rushing 2006; Buswell et al. 2011) or niche shifts from correlative SDMs (Godsoe \& Case 2014), this can be greatly exacerbated when combining these two methods. Although there may be underlying patterns relating trait change in introduced plants and climate niche shifts, it would likely require many more replicate species than the number used in the meta-regression analysis of this study to properly identify them.

\subsubsection{Characteristics of realised niche shifts}

Species introductions to New Zealand underwent a greater magnitude of realised niche shift than those into Australia, measured in in terms of niche expansion, total niche change, and degree of niche overlap. Niche shifts into New Zealand tended to represent an expansion into a wetter climate, while shifts into Australia were often a shift into warmer climate space. In the original study examining trait change through time in these species, the results of Buswell et al. (2011) indicated that plants would tend to show a decline in height through time in the more arid, inland region of NSW, which was suggested to be likely driven by a lack of moisture availability. However, the results of the current study are counter to this idea because i) species introduced to Australia did not tend to show a decline in height through time, when location and climate variables were controlled for in the model, and ii) very few species introduced to Australia underwent a niche shifts into drier climate, as hypothesised by Buswell et al. (2011).

\subsubsection{Assessment using analog and non-analog climates}

When niche shifts were assessed using only analog climate space shared between the two ranges, niche shift measures of niche expansion and total niche change were greatly reduced. However, there was still no clear difference in the ability of niche shifts to predict trait change through time when measured using all available non-analog climate space, or only analog climate 
space shared between the two ranges. It is possible that niche shifts were poor at predicting trait change through time, because niche shifts in analog climate space were not large enough to then drive a change in traits, particularly for species introduced to Australia. This would support the idea put forward by Petitpierre et al. (2012), stating that shifts into non-analog climate space cannot be considered as 'true' niche shifts, as they may only signify the expansion of a plant into climate space it is already pre-adapted to (i.e., a demonstration of the latent fundamental niche). If the niche shifts observed in this study did largely represent a plant's expansion into climate space that it is already adapted to, then this could be why the magnitude of climate niche shift poorly predicted the rate of trait change through time.

\subsection{Summary}

Realised climate niche shifts did not effectively predict herbarium plant trait change through time across multiple species in this study. This suggests that climate (specifically soil moisture and the temperature of the warmest quarter) may not be a predominant driver of trait change in introduced plants in Australia and New Zealand. Alternatively, the combined noise and the mismatch in scales that may arise when combining these two methods (i.e., trait change through time predicted from herbarium specimens, and niche shifts predicted from correlative SDMs) could mask any underlying patterns in plant trait responses to the new environment. While the use of herbarium specimens and species distribution models may individually provide useful tools to explore species responses to shifts in geographic range and climate, the two approaches did not combine to provide a consistent perspective on how these processes affect species that have expanded into introduced ranges where they may experience novel abiotic conditions. 


\subsection{Appendix B - Supplementary Material}

Table S9. Niche shift metrics used in meta-analysis to predict trait change through time, for each species introduced to Australia. The niche centroid shift along a 2-D axis refers to the shift in climate space along both rescaled axis' (with each axis normalised between $0-100)$. Niche expansion and total niche change metrics are given at the threshold of $99^{\text {th }}$ percentile of marginal climate space.

\begin{tabular}{|c|c|c|c|c|c|c|c|c|}
\hline \multirow{2}{*}{ Species } & \multicolumn{2}{|c|}{ Niche Expansion } & \multicolumn{2}{|c|}{ Total Niche Change } & \multirow[t]{2}{*}{ Schoeners' D } & \multicolumn{3}{|c|}{ Niche Centroid Shift } \\
\hline & Analog & Non-analog & Analog & Non-analog & & Temperature $\left({ }^{\circ} \mathrm{C}\right)$ & Moisture & 2-D axis \\
\hline Arctotheca populifolia & 0.003 & 0.278 & 0.021 & 0.308 & 0.136 & -0.839 & 0.033 & 4.517 \\
\hline Arenaria leptoclados & 0.019 & 0.016 & 0.265 & 0.287 & 0.410 & 3.459 & -0.184 & 16.925 \\
\hline Cicendia quadrangularis & 0.170 & 0.170 & 0.497 & 0.688 & 0.169 & -0.029 & 0.161 & 8.487 \\
\hline Euphorbia peplus & 0.166 & 0.131 & 0.186 & 0.291 & 0.217 & 3.799 & -0.201 & 18.380 \\
\hline Facelis retusa & 0.000 & 0.062 & 0.296 & 0.543 & 0.204 & -0.610 & -0.032 & 3.029 \\
\hline Gamochaeta americana & 0.000 & 0.042 & 0.204 & 0.500 & 0.274 & 1.320 & -0.112 & 5.929 \\
\hline Geranum molle & 0.061 & 0.044 & 0.145 & 0.167 & 0.417 & 2.245 & -0.184 & 15.812 \\
\hline Medicago lupulina & 0.128 & 0.113 & 0.234 & 0.271 & 0.206 & 3.318 & -0.180 & 14.692 \\
\hline Medicago polymorpha & 0.043 & 0.037 & 0.148 & 0.230 & 0.548 & 2.334 & -0.066 & 7.837 \\
\hline Melilotus indicus & 0.007 & 0.015 & 0.169 & 0.322 & 0.396 & 3.395 & -0.203 & 16.910 \\
\hline Petrorhagia nanteullii & 0.068 & 0.065 & 0.295 & 0.303 & 0.334 & -0.248 & 0.160 & 12.549 \\
\hline Polycarpon tetraphyllum & 0.051 & 0.050 & 0.064 & 0.131 & 0.547 & 0.447 & 0.041 & 3.470 \\
\hline Silene gallica & 0.025 & 0.019 & 0.069 & 0.172 & 0.549 & 1.667 & -0.087 & 8.030 \\
\hline Stachys arvensis & 0.364 & 0.391 & 0.484 & 0.587 & 0.221 & 5.862 & -0.236 & 25.102 \\
\hline Trifolium dubium & 0.056 & 0.052 & 0.089 & 0.150 & 0.439 & 3.517 & -0.265 & 23.332 \\
\hline Trifolium glomeratum & 0.040 & 0.036 & 0.071 & 0.128 & 0.650 & 1.248 & -0.020 & 3.957 \\
\hline Veronica arvensis & 0.093 & 0.085 & 0.203 & 0.228 & 0.479 & 3.338 & -0.220 & 18.528 \\
\hline
\end{tabular}


Table S10. Niche shift metrics used in meta-analysis to predict trait change through time, for each species introduced to New Zealand. The niche centroid shift along a 2-D axis refers to the shift in climate space along both rescaled axis' (with each axis normalised between $0-100)$. Niche expansion and total niche change metrics are given at the threshold of $99^{\text {th }}$ percentile of marginal climate space.

\begin{tabular}{|c|c|c|c|c|c|c|c|c|}
\hline \multirow{2}{*}{ Species } & \multicolumn{2}{|c|}{ Niche Expansion } & \multicolumn{2}{|c|}{ Total Niche Change } & \multirow[t]{2}{*}{ Schoeners' D } & \multicolumn{3}{|c|}{ Niche Centroid Shift } \\
\hline & Analog & Non-analog & Analog & Non-analog & & Temperature $\left({ }^{\circ} \mathrm{C}\right)$ & Moisture & 2-D axis \\
\hline Acaena agnipila & 0.302 & 0.482 & 0.373 & 0.982 & 0.443 & -3.138 & 0.419 & 28.21 \\
\hline Carex longebrachiata & 0.034 & 0.363 & 0.709 & 1.141 & 0.365 & -1.359 & 0.347 & 22.16 \\
\hline Cerastium glomeratum & 0.216 & 0.310 & 0.217 & 0.478 & 0.277 & -1.025 & 0.319 & 18.06 \\
\hline Euphorbia peplus & 0.590 & 0.632 & 0.594 & 0.774 & 0.404 & 0.381 & 0.286 & 16.11 \\
\hline Lythrum hyssopifolia & 0.590 & 0.632 & 0.720 & 1.034 & 0.302 & -1.550 & 0.487 & 27.77 \\
\hline Medicago lupulina & 0.215 & 0.271 & 0.216 & 0.366 & 0.204 & 0.213 & 0.186 & 19.75 \\
\hline Melilotus indicus & 0.118 & 0.173 & 0.125 & 0.557 & 0.256 & -1.307 & 0.344 & 19.57 \\
\hline Myosotis arvensis & 0.188 & 0.324 & 0.188 & 0.372 & 0.233 & -0.371 & 0.255 & 14.56 \\
\hline Persicaria maculosa & 0.360 & 0.426 & 0.377 & 0.464 & 0.192 & 1.442 & 0.179 & 10.80 \\
\hline Plantago coronopus & 0.266 & 0.325 & 0.268 & 0.657 & 0.296 & -1.693 & 0.363 & 20.95 \\
\hline Poa annua & 0.194 & 0.346 & 0.217 & 0.485 & 0.268 & 0.175 & 0.324 & 18.17 \\
\hline Ranunculus parviflorus & 0.295 & 0.355 & 0.340 & 0.673 & 0.346 & -0.968 & 0.375 & 21.36 \\
\hline Silene gallica & 0.234 & 0.329 & 0.234 & 0.787 & 0.299 & -2.473 & 0.451 & 26.15 \\
\hline Solenogyne gunni & 0.247 & 0.518 & 0.290 & 0.674 & 0.312 & -0.990 & 0.351 & 21.35 \\
\hline Stachys arvensis & 0.268 & 0.009 & 0.277 & 0.104 & 0.222 & 0.603 & 0.226 & 12.79 \\
\hline Trifolium dubium & 0.304 & 0.387 & 0.307 & 0.457 & 0.231 & -0.647 & 0.277 & 15.77 \\
\hline Vicia tetrasperma & 0.476 & 0.508 & 0.566 & 0.651 & 0.202 & 0.596 & 0.309 & 17.46 \\
\hline
\end{tabular}


Table S11. The rate of trait change through time for species introduced to Australia. The beta-coefficient $(B)$ for each trait is the slope of the year term in a GLS model. $10^{(B \times 100)}$ represents the proportional rate of trait change through time over a 100 year period.

\begin{tabular}{|c|c|c|c|c|c|c|c|c|c|c|c|c|}
\hline \multirow{2}{*}{ Species } & \multicolumn{4}{|c|}{ Height } & \multicolumn{4}{|c|}{ Leaf Area } & \multicolumn{4}{|c|}{ Leaf Shape } \\
\hline & $B$ & S.E. & $n$ & $10^{(B \times 100)}$ & $B$ & S.E. & $n$ & $\left.10^{(B \times 100)}\right)$ & $B$ & S.E. & $n$ & $10^{(B \times 100)}$ \\
\hline A. populifolia & 0.00180 & 0.00145 & 36 & 1.51 & -0.00033 & 0.00196 & 52 & 0.93 & 0.00096 & 0.00066 & 52 & 1.25 \\
\hline A. leptoclados & 0.00115 & 0.00107 & 115 & 1.30 & -0.00004 & 0.00169 & 122 & 0.99 & 0.00010 & 0.00051 & 122 & 1.02 \\
\hline C. quadrangularis & -0.00068 & 0.00075 & 122 & 0.86 & 0.00392 & 0.00154 & 111 & 2.46 & -0.00094 & 0.00055 & 111 & 0.81 \\
\hline E. peplus & 0.00051 & 0.00046 & 91 & 1.12 & 0.00020 & 0.00111 & 69 & 1.05 & -0.00082 & 0.00040 & 69 & 0.83 \\
\hline F. retusa & -0.00416 & 0.00247 & 58 & 0.38 & & & & & & & & \\
\hline G. americana & 0.00020 & 0.00113 & 139 & 1.05 & 0.00032 & 0.00204 & 141 & 1.08 & -0.00133 & 0.00058 & 141 & 0.74 \\
\hline G. molle & -0.00063 & 0.00119 & 107 & 0.87 & 0.00509 & 0.00213 & 28 & 3.23 & 0.00062 & 0.00046 & 28 & 1.15 \\
\hline M. Iupulina & 0.00145 & 0.00155 & 54 & 1.40 & -0.00045 & 0.00134 & 57 & 0.90 & -0.00041 & 0.00038 & 70 & 0.91 \\
\hline M. polymorpha & 0.00115 & 0.00107 & 62 & 1.30 & -0.00098 & 0.00119 & 120 & 0.80 & -0.00014 & 0.00022 & 120 & 0.97 \\
\hline M. indicus & 0.00340 & 0.00304 & 53 & 2.19 & 0.00340 & 0.00304 & 35 & 2.19 & -0.00055 & 0.00040 & 36 & 0.88 \\
\hline P. nanteullii & -0.00009 & 0.00114 & 137 & 0.98 & & & & & & & & \\
\hline P. tetraphyllum & -0.00176 & 0.00112 & 103 & 0.67 & -0.00222 & 0.00089 & 49 & 0.60 & -0.00053 & 0.00040 & 103 & 0.89 \\
\hline S. gallica & -0.00053 & 0.00092 & 74 & 0.88 & -0.00068 & 0.00094 & 78 & 0.85 & -0.00055 & 0.00032 & 78 & 0.88 \\
\hline S. arvensis & 0.00043 & 0.00093 & 182 & 1.10 & -0.00123 & 0.00155 & 107 & 0.75 & -0.00004 & 0.00023 & 107 & 0.99 \\
\hline T. dubium & -0.00083 & 0.00175 & 102 & 0.83 & 0.00033 & 0.00117 & 117 & 1.08 & 0.00042 & 0.00029 & 117 & 1.10 \\
\hline T. glomeratum & 0.00266 & 0.00100 & 129 & 1.85 & -0.00050 & 0.00098 & 76 & 0.89 & -0.00041 & 0.00028 & 76 & 0.91 \\
\hline V. arvensis & & & & & -0.00464 & 0.00120 & 67 & 0.34 & 0.00160 & 0.00036 & 67 & 1.44 \\
\hline
\end{tabular}


Table S12. The rate of trait change through time for species introduced to New Zealand. The beta-coefficient $(B)$ for each trait is the slope of the year term in a GLS model. $10^{(B \times 100)}$ represents the proportional rate of trait change through time over a 100 year period.

\begin{tabular}{|c|c|c|c|c|c|c|c|c|c|c|c|c|}
\hline \multirow{2}{*}{ Species } & \multicolumn{4}{|c|}{ Height } & \multicolumn{4}{|c|}{ Leaf Area } & \multicolumn{4}{|c|}{ Leaf Shape } \\
\hline & $B$ & S.E. & $n$ & $10^{(B \times 100)}$ & $B$ & S.E. & $n$ & $\left.10^{(B \times 100)}\right)$ & $B$ & S.E. & $n$ & $10^{(B \times 100)}$ \\
\hline A. agnipila & 0.00140 & 0.00095 & 78 & 1.38 & 0.00137 & 0.00084 & 82 & 1.37 & -0.00028 & 0.00031 & 82 & 0.94 \\
\hline C. longebrachiata & -0.00013 & 0.00138 & 35 & 0.97 & & & & & & & & \\
\hline C. glomeratum & -0.00311 & 0.00230 & 129 & 0.49 & -0.00320 & 0.00118 & 139 & 0.48 & 0.00025 & 0.00028 & 139 & 1.06 \\
\hline E. peplus & -0.00056 & 0.00066 & 166 & 0.88 & -0.00050 & 0.00120 & 141 & 0.89 & -0.00006 & 0.00031 & 141 & 0.99 \\
\hline L. hyssopifolia & & & & & 0.00048 & 0.00136 & 45 & 1.12 & -0.00053 & 0.00055 & 45 & 0.88 \\
\hline M. Iupulina & & & & & -0.00521 & 0.00181 & 57 & 0.30 & -0.00069 & 0.00059 & 57 & 0.85 \\
\hline M. indicus & & & & & 0.00057 & 0.00128 & 69 & 1.14 & 0.00043 & 0.00053 & 69 & 1.10 \\
\hline M. arvensis & 0.00031 & 0.00125 & 91 & 1.07 & 0.00376 & 0.00162 & 89 & 2.38 & 0.00172 & 0.00065 & 89 & 1.48 \\
\hline P. maculosa & & & & & 0.00009 & 0.00224 & 42 & 1.02 & -0.00056 & 0.00107 & 42 & 0.88 \\
\hline P. coronopus & -0.00120 & 0.00085 & 155 & 0.76 & & & & & & & & \\
\hline P. annua & -0.00379 & 0.00174 & 28 & 0.42 & & & & & & & & \\
\hline R. parviflorus & -0.00171 & 0.00143 & 101 & 0.67 & -0.00022 & 0.00157 & 96 & 0.95 & -0.00009 & 0.00022 & 96 & 0.98 \\
\hline S. gallica & -0.00119 & 0.00102 & 199 & 0.76 & -0.00503 & 0.00195 & 141 & 0.31 & 0.00156 & 0.00059 & 143 & 1.43 \\
\hline S. gunni & -0.00049 & 0.00106 & 57 & 0.89 & 0.00389 & 0.00116 & 55 & 2.45 & 0.00006 & 0.00026 & 58 & 1.01 \\
\hline S. arvensis & 0.00041 & 0.00096 & 72 & 1.10 & -0.00189 & 0.00133 & 69 & 0.65 & -0.00038 & 0.00029 & 69 & 0.92 \\
\hline T. dubium & -0.00127 & 0.00191 & 111 & 0.75 & -0.00328 & 0.00134 & 137 & 0.47 & 0.00003 & 0.00028 & 63 & 1.01 \\
\hline V. tetrasperma & & & & & 0.00053 & 0.00098 & 35 & 1.13 & -0.00030 & 0.00061 & 35 & 0.93 \\
\hline
\end{tabular}


Figures S9 - S42. The climatic niche calculated for the native (top) and introduced (bottom) ranges of each introduced species to Australia and New Zealand. Left panels: Red (native) and blue (introduced) dots represent the locations for the native and introduced locations respectively, surrounding grey shading show the $800 \mathrm{~km}$ buffer in which background climate points were taken from. Middle panels: Red and blue dots represent the location of presences in climate space, grey dots represent the background points in climate space. Right panels: Solid lines represent the extent of the available climate space at the $99^{\text {th }}$ percentile for the native range (red) and introduced range (blue), dashed lines show this at the 75th percentile; colored areas show the extent of climate space occupied in only the native range (red; niche unfilling), the introduced range (blue; niche expansion) and their overlap (purple; niche conserved); shading (greyscale) represents the densities (species occupancy) of the present locations in climate space for the native (top) and introduced (bottom) ranges; crosses (top panel) show the niche centroid for the native (red) and introduced ranges (blue); black circles (bottom panel) show the location in climate space of the herbarium specimens used to model trait change through time.

Tables S12 - S45. Niche shift metrics for niche expansion and niche unfilling, in analog and non-analog climates, for each species introduced to Australia and New Zealand. Niche shift metrics are calculated at a range thresholds for the percentile of marginal climate space to include $(75-99 \%)$ 

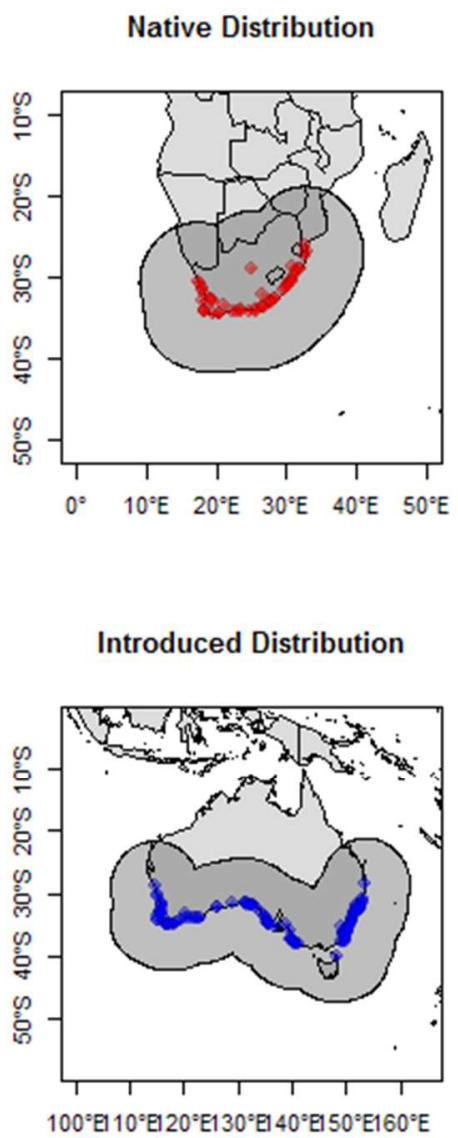

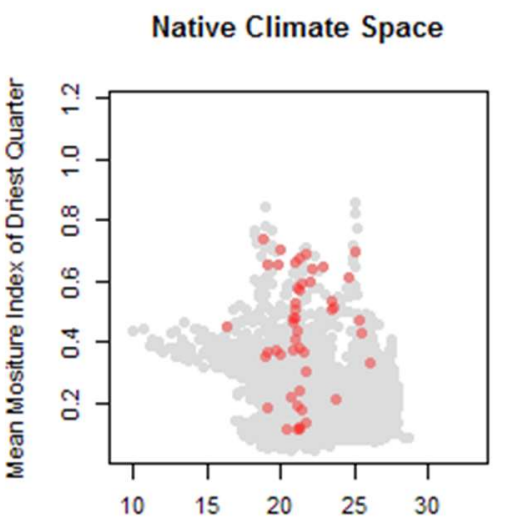

Mean Temperature of Warmest Quarter

Introduced Climate Space

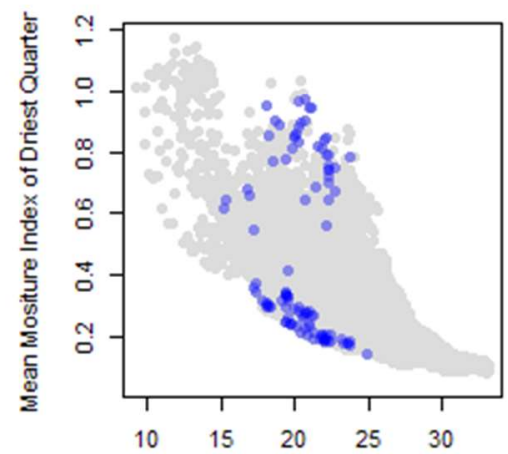

Mean Temperature of Warmest Quarter
Native Climate Densities

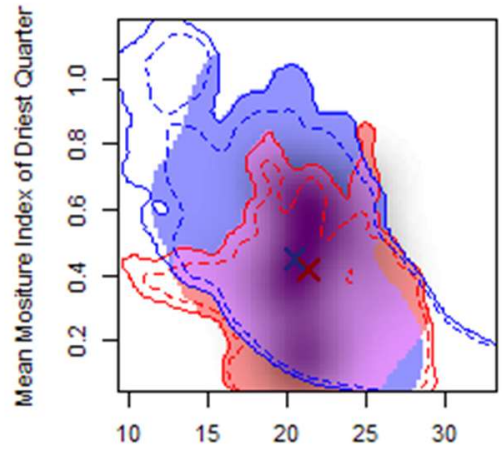

Mean Temperature of Warmest Quarte

Introduced Climate Densities

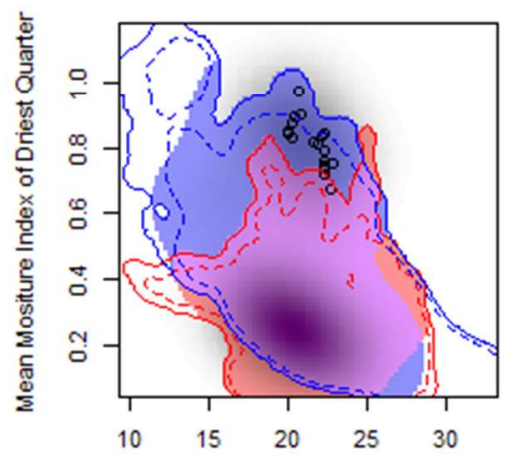

Mean Temperature of Warmest Quarter

Figure S9. Introduction of Arctotheca populifolia to Australia

Table S13. Niche shift indices for the introduction of Arctotheca populifolia to Australia

\begin{tabular}{cccccc}
\hline \multirow{2}{*}{$\begin{array}{c}\text { Marginal } \\
\text { climate }\end{array}$} & \multicolumn{2}{c}{ Niche Expansion } & & \multicolumn{2}{c}{ Niche Unfilling } \\
\cline { 2 - 3 } \cline { 5 - 6 } threshold (\%) & $\begin{array}{c}\text { Analog } \\
\text { climate }\end{array}$ & $\begin{array}{c}\text { Non-analog } \\
\text { climate }\end{array}$ & & $\begin{array}{c}\text { Analog } \\
\text { climate }\end{array}$ & $\begin{array}{c}\text { Non-analog } \\
\text { climate }\end{array}$ \\
\hline 99 & 0.003 & 0.249 & & 0.018 & 0.058 \\
95 & 0.003 & 0.257 & & 0.018 & 0.061 \\
90 & 0.004 & 0.263 & & 0.017 & 0.065 \\
85 & 0.004 & 0.267 & & 0.017 & 0.069 \\
80 & 0.004 & 0.271 & & 0.017 & 0.075 \\
75 & 0.004 & 0.278 & & 0.016 & 0.083 \\
\hline
\end{tabular}


Native Distribution

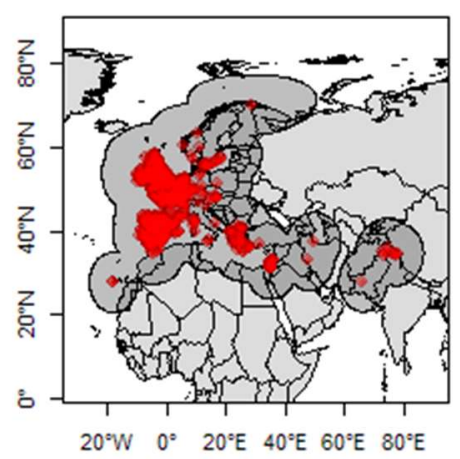

Introduced Distribution

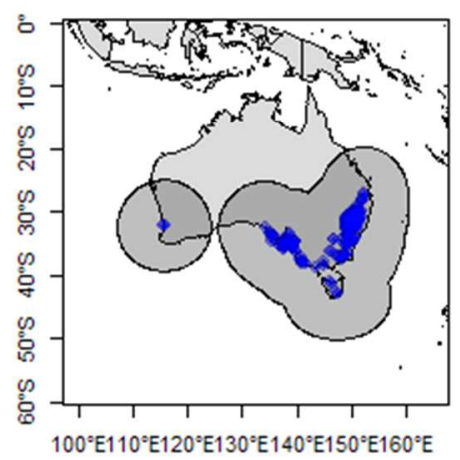

Native Climate Space

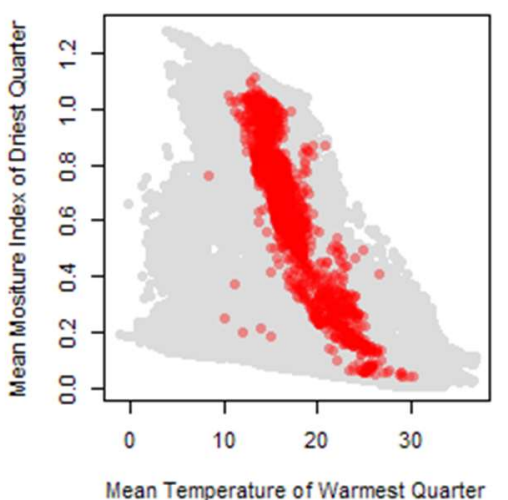

Introduced Climate Space

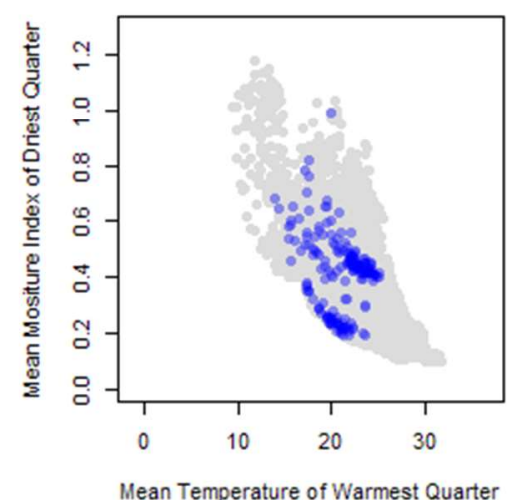

Native Climate Densities

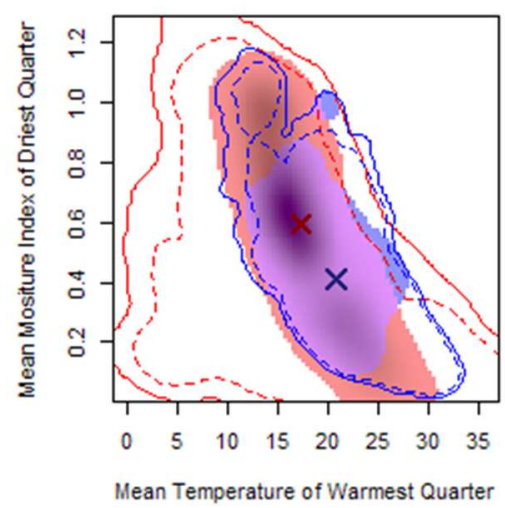

Introduced Climate Densities

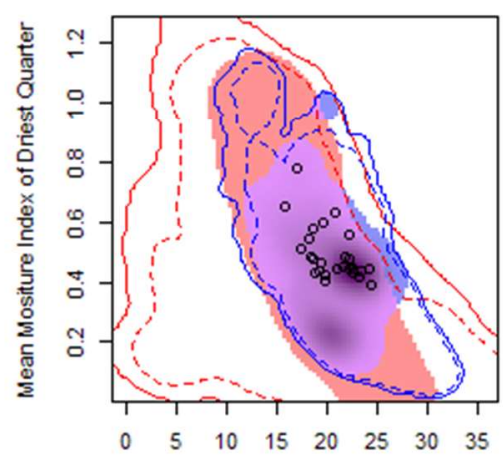

Mean Temperature of Warmest Quarter

Figure S10. Introduction of Arenaria leptoclados to Australia

Table S14. Niche shift indices for the introduction of Arenaria leptoclados to Australia

\begin{tabular}{cccccc}
\hline \multirow{2}{*}{$\begin{array}{c}\text { Marginal } \\
\text { climate }\end{array}$} & \multicolumn{2}{c}{ Niche Expansion } & & \multicolumn{2}{c}{ Niche Unfilling } \\
\cline { 2 - 3 } \cline { 5 - 6 } threshold $(\%)$ & $\begin{array}{c}\text { Analog } \\
\text { climate }\end{array}$ & $\begin{array}{c}\text { Non-analog } \\
\text { climate }\end{array}$ & & $\begin{array}{c}\text { Analog } \\
\text { climate }\end{array}$ & $\begin{array}{c}\text { Non-analog } \\
\text { climate }\end{array}$ \\
\hline 99 & 0.019 & 0.019 & & 0.246 & 0.268 \\
95 & 0.019 & 0.019 & & 0.236 & 0.269 \\
90 & 0.018 & 0.018 & & 0.225 & 0.270 \\
85 & 0.017 & 0.018 & & 0.212 & 0.273 \\
80 & 0.013 & 0.017 & & 0.195 & 0.279 \\
75 & 0.005 & 0.016 & & 0.170 & 0.285 \\
\hline
\end{tabular}



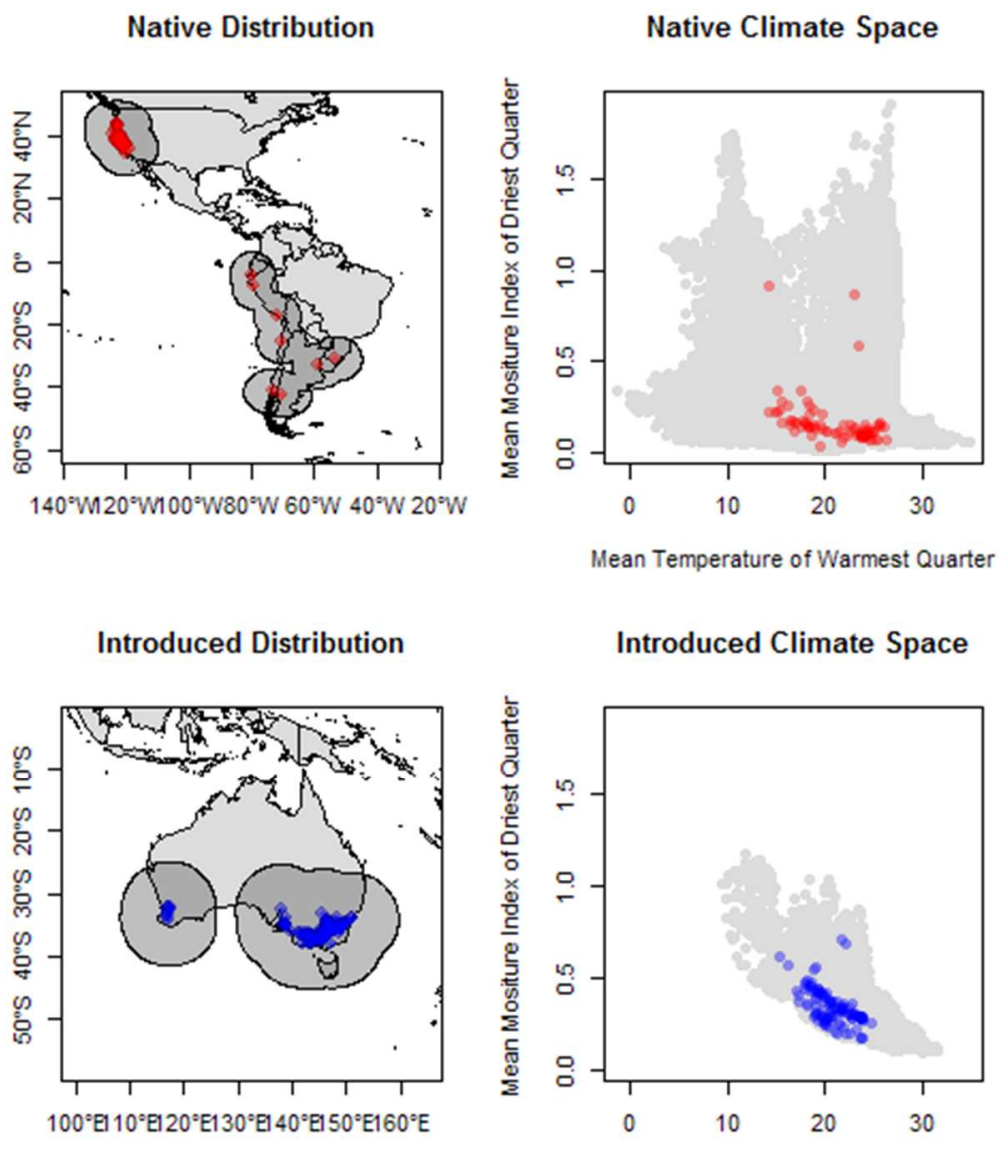

Mean Temperature of Warmest Quarter

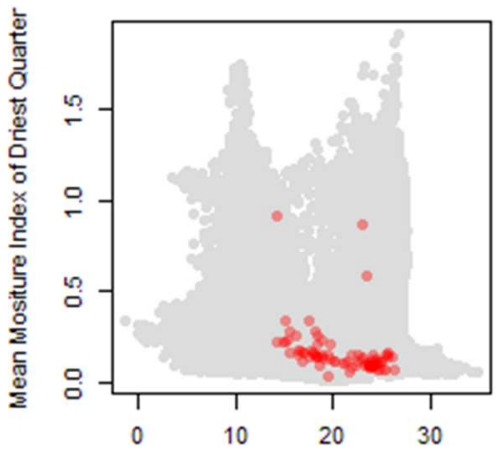

Native Climate Densities

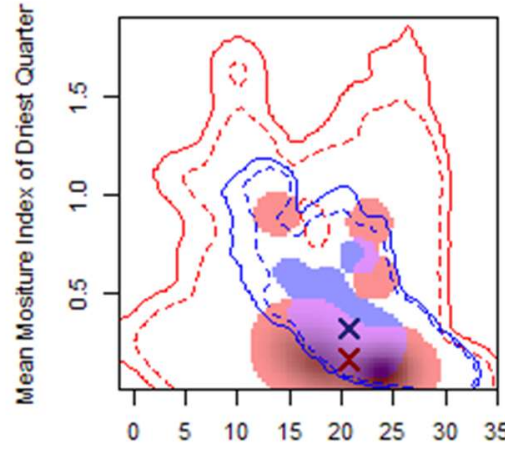

Mean Temperature of Warmest Quarter

Introduced Climate Densities

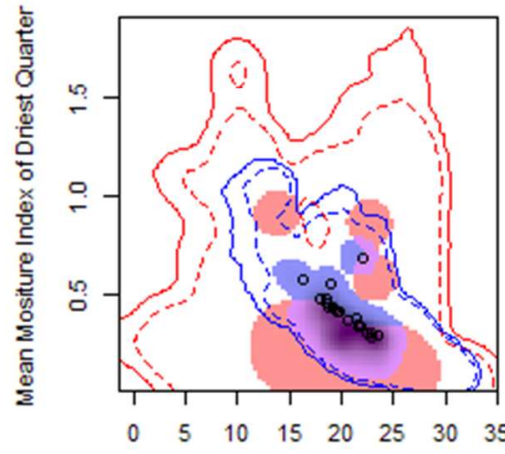

Mean Temperature of Warmest Quarter

Figure S11. Introduction of Cicendia quadrangularis to Australia

Table S15. Niche shift indices for the introduction of Cicendia quadrangularis to Australia

\begin{tabular}{cccccc}
\hline \multirow{2}{*}{$\begin{array}{c}\text { Marginal } \\
\text { climate }\end{array}$} & \multicolumn{2}{c}{ Niche Expansion } & & \multicolumn{2}{c}{ Niche Unfilling } \\
\cline { 2 - 3 } \cline { 5 - 6 } threshold (\%) & $\begin{array}{c}\text { Analog } \\
\text { climate }\end{array}$ & $\begin{array}{c}\text { Non-analog } \\
\text { climate }\end{array}$ & & $\begin{array}{c}\text { Analog } \\
\text { climate }\end{array}$ & $\begin{array}{c}\text { Non-analog } \\
\text { climate }\end{array}$ \\
\hline 99 & 0 & 0.005 & & 0.296 & 0.538 \\
95 & 0 & 0.012 & & 0.285 & 0.550 \\
90 & 0 & 0.023 & & 0.285 & 0.567 \\
85 & 0 & 0.037 & & 0.286 & 0.582 \\
80 & 0 & 0.049 & & 0.288 & 0.600 \\
75 & 0 & 0.062 & & 0.293 & 0.619 \\
\hline
\end{tabular}




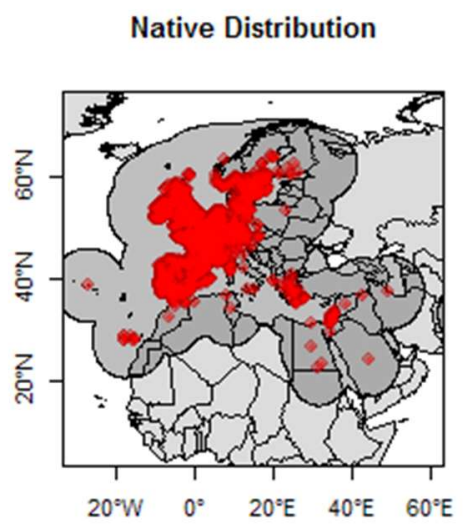

Introduced Distribution

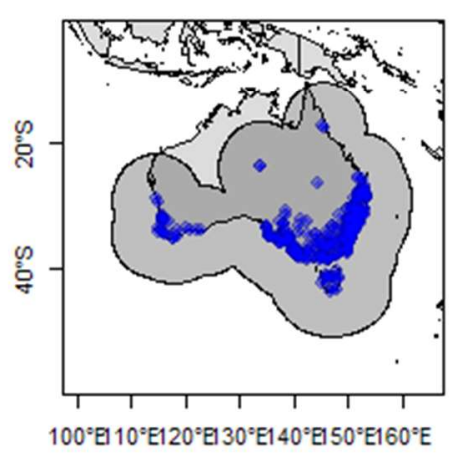

Native Climate Space

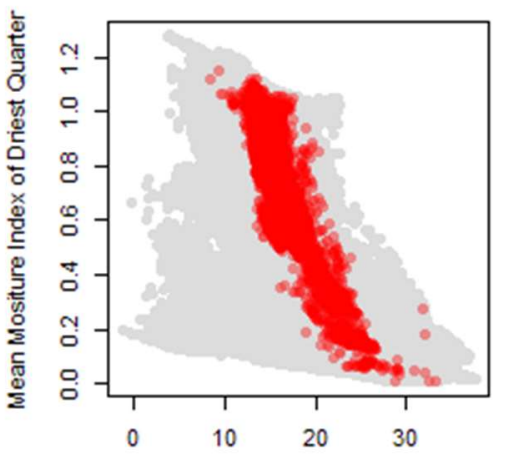

Mean Temperature of Warmest Quarter

Introduced Climate Space

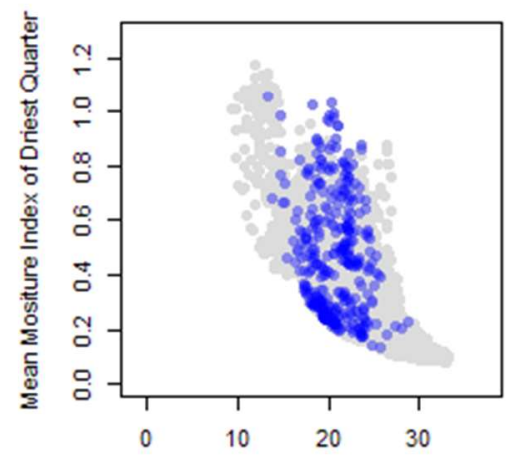

Mean Temperature of Warmest Quarter
Native Climate Densities

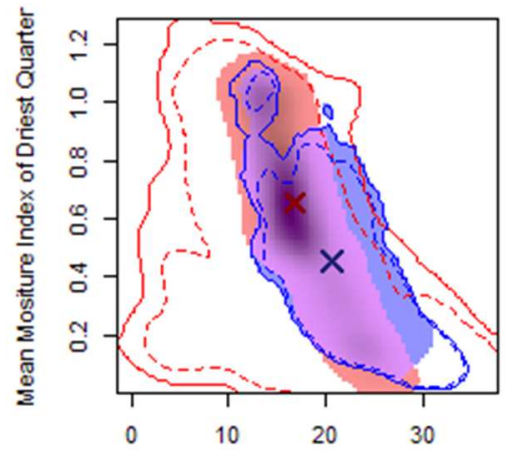

Mean Temperature of Warmest Quarter

Introduced Climate Densities

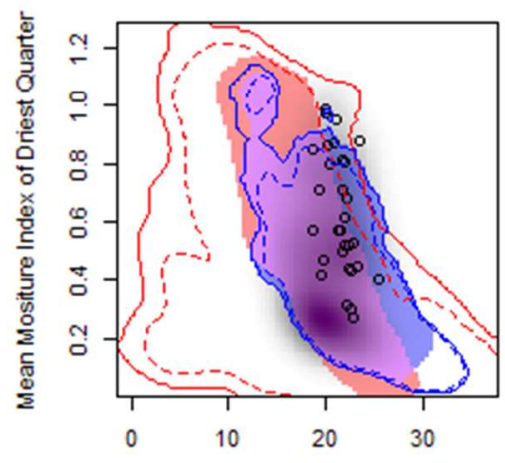

Mean Temperature of Warmest Quarter

Figure S12. Introduction of Euphorbia peplus to Australia

Table S16. Niche shift indices for the introduction of Euphorbia peplus to Australia

\begin{tabular}{cccccc}
\hline \multirow{2}{*}{$\begin{array}{c}\text { Marginal } \\
\text { climate } \\
\text { threshold }(\%)\end{array}$} & \multicolumn{2}{c}{\begin{tabular}{c} 
Niche Expansion \\
\cline { 2 - 3 } climate
\end{tabular}} & $\begin{array}{c}\text { Non-analog } \\
\text { climate }\end{array}$ & & \multicolumn{2}{c}{$\begin{array}{c}\text { Analog } \\
\text { climate }\end{array}$} & $\begin{array}{c}\text { Non-analog } \\
\text { climate }\end{array}$ \\
\hline 99 & 0.166 & 0.173 & & 0.019 & 0.119 \\
95 & 0.153 & 0.165 & & 0.016 & 0.146 \\
90 & 0.138 & 0.157 & & 0.011 & 0.175 \\
85 & 0.120 & 0.149 & & 0.005 & 0.210 \\
80 & 0.100 & 0.140 & & 0.001 & 0.251 \\
75 & 0.077 & 0.131 & & 0.001 & 0.302 \\
\hline
\end{tabular}



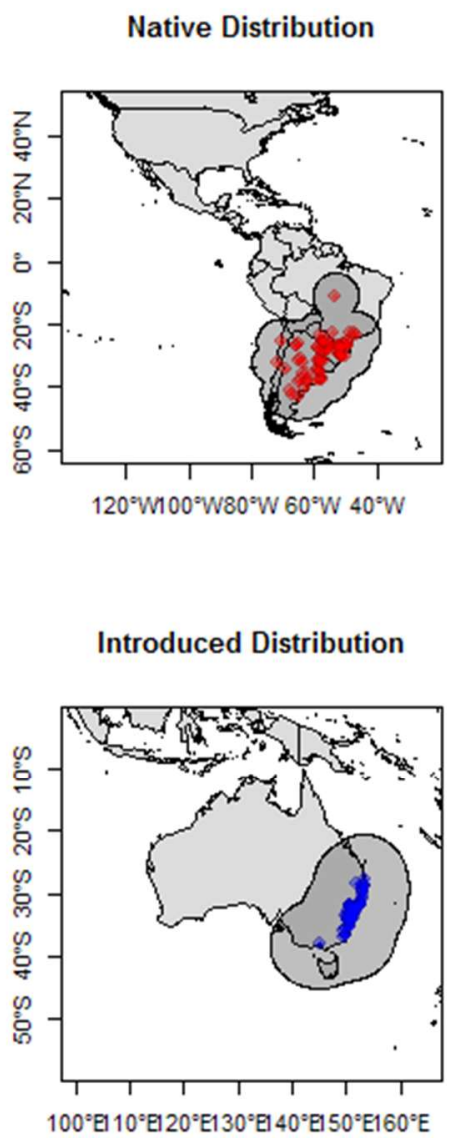

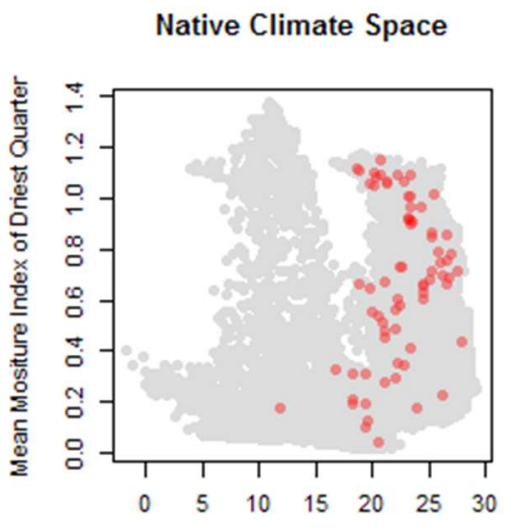

Mean Temperature of Warmest Quarter

Introduced Climate Space

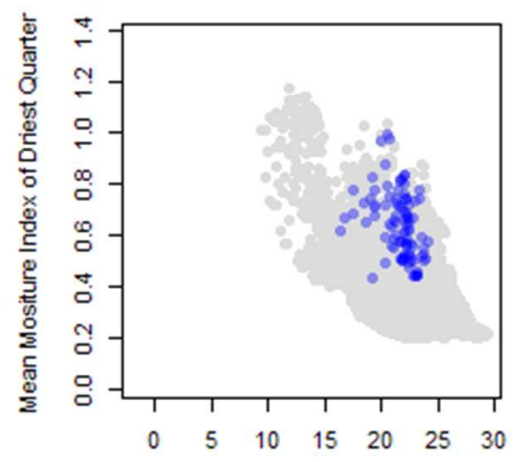

Mean Temperature of Warmest Quarter
Native Climate Densities

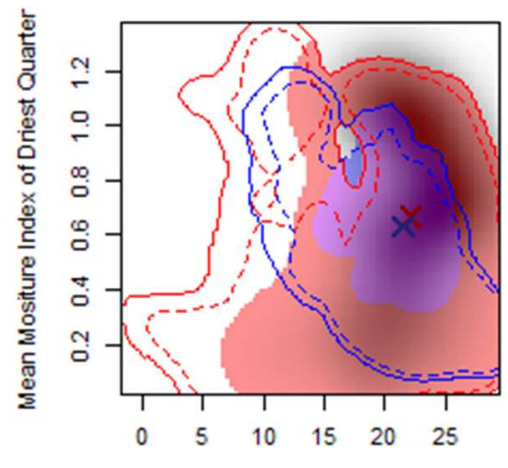

Mean Temperature of Warmest Quarter

Introduced Climate Densities

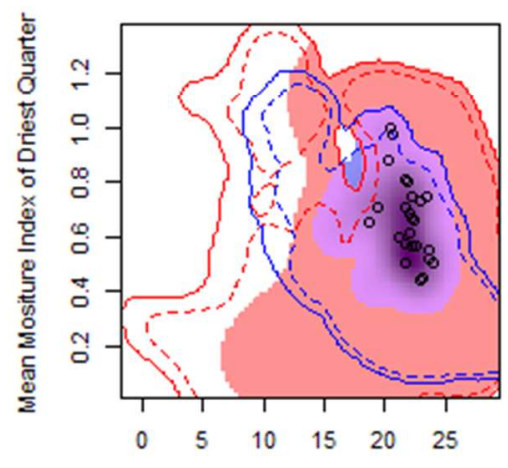

Mean Temperature of Warmest Quarter

Figure S13. Facelis retusa introduction to Australia

Table S17. Niche shift indices for the introduction of Facelis retusa to Australia

\begin{tabular}{cccccc}
\hline \multirow{2}{*}{$\begin{array}{c}\text { Marginal } \\
\text { climate } \\
\text { threshold }(\%)\end{array}$} & \multicolumn{2}{c}{$\begin{array}{c}\text { Niche Expansion } \\
\text { climate }\end{array}$} & $\begin{array}{c}\text { Non-analog } \\
\text { climate }\end{array}$ & & \multicolumn{2}{c}{$\begin{array}{c}\text { Analog } \\
\text { climate }\end{array}$} & $\begin{array}{c}\text { Non-analog } \\
\text { climate }\end{array}$ \\
\cline { 2 - 3 } 99 & 0 & 0.005 & & 0.296 & 0.538 \\
95 & 0 & 0.012 & & 0.285 & 0.550 \\
90 & 0 & 0.023 & & 0.285 & 0.567 \\
85 & 0 & 0.037 & & 0.286 & 0.582 \\
80 & 0 & 0.049 & & 0.288 & 0.600 \\
75 & 0 & 0.062 & & 0.293 & 0.619 \\
\hline
\end{tabular}



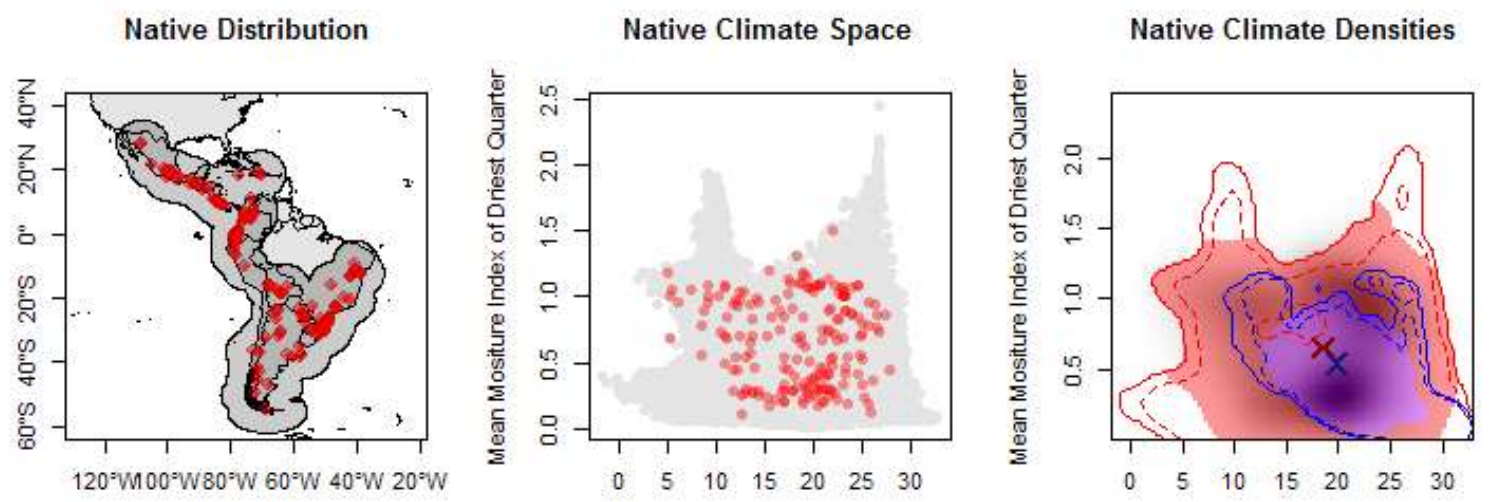

Mean Temperature of Warmest Quarter

Mean Temperature of Warmest Quarter

Introduced Distribution

Introduced Climate Space
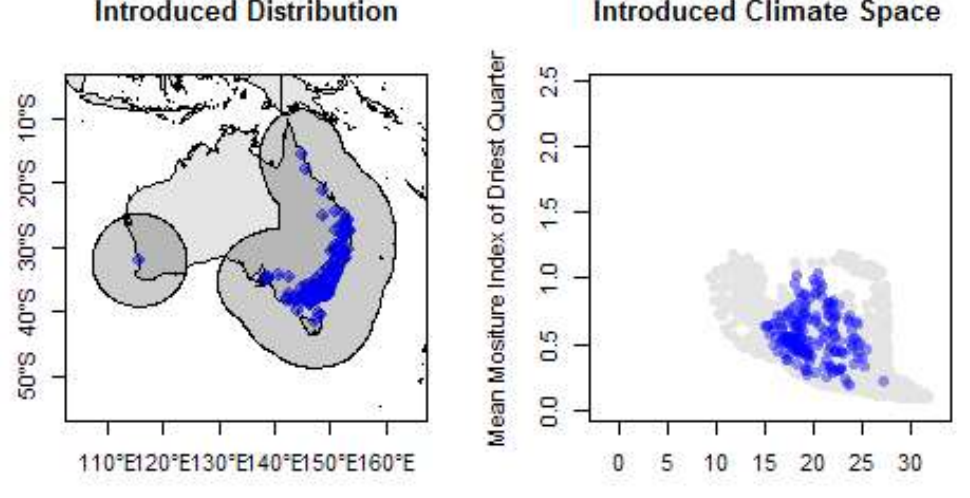

Introduced Climate Densities

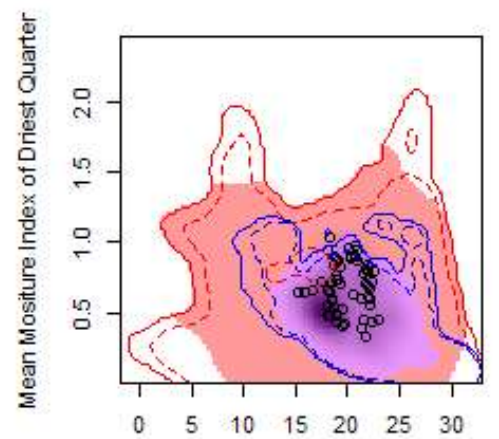

Mean Temperature of Warmest Quarter

Mean Temperature of Warmest Quarter

Figure S14. Introduction of Gamochaeta americana to Australia

Table S18. Niche shift indices for the introduction of Gamochaeta americana to Australia

\begin{tabular}{|c|c|c|c|c|}
\hline \multirow{2}{*}{$\begin{array}{c}\text { Marginal } \\
\text { climate } \\
\text { threshold }(\%)\end{array}$} & \multicolumn{2}{|c|}{ Niche Expansion } & \multicolumn{2}{|c|}{ Niche Unfilling } \\
\hline & $\begin{array}{l}\text { Analog } \\
\text { climate }\end{array}$ & $\begin{array}{l}\text { Non-analog } \\
\text { climate }\end{array}$ & $\begin{array}{l}\text { Analog } \\
\text { climate }\end{array}$ & $\begin{array}{l}\text { Non-analog } \\
\text { climate }\end{array}$ \\
\hline 99 & 0 & 0 & 0.204 & 0.500 \\
\hline 95 & 0 & 0 & 0.185 & 0.507 \\
\hline 90 & 0 & 0 & 0.168 & 0.517 \\
\hline 85 & 0 & 0 & 0.146 & 0.524 \\
\hline 80 & 0 & 0.004 & 0.129 & 0.536 \\
\hline 75 & 0 & 0.042 & 0.095 & 0.550 \\
\hline
\end{tabular}




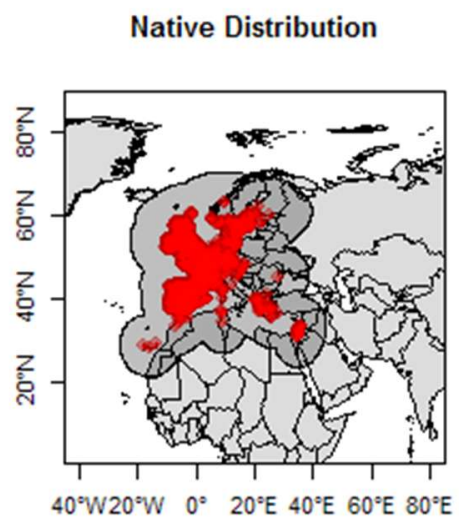

Introduced Distribution

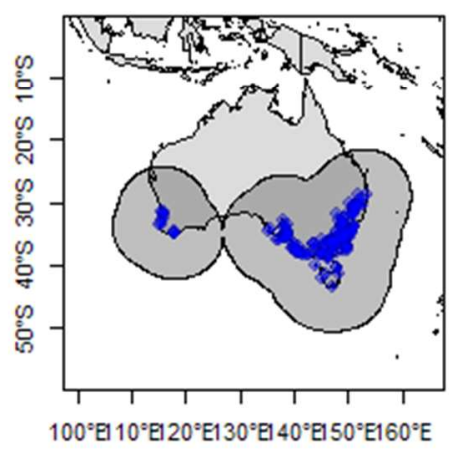

Native Climate Space

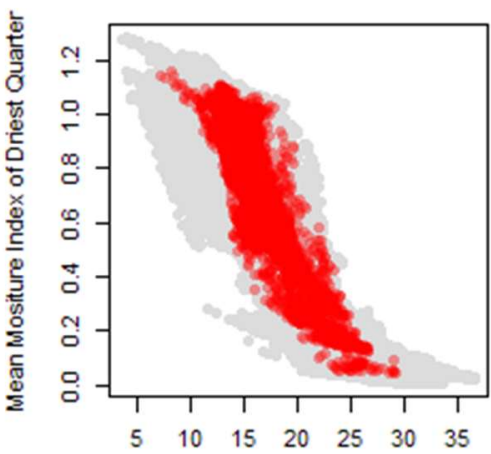

Mean Temperature of Warmest Quarter

Introduced Climate Space

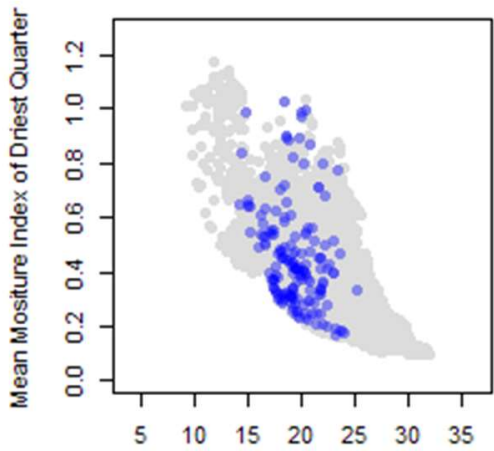

Mean Temperature of Warmest Quarter
Native Climate Densities

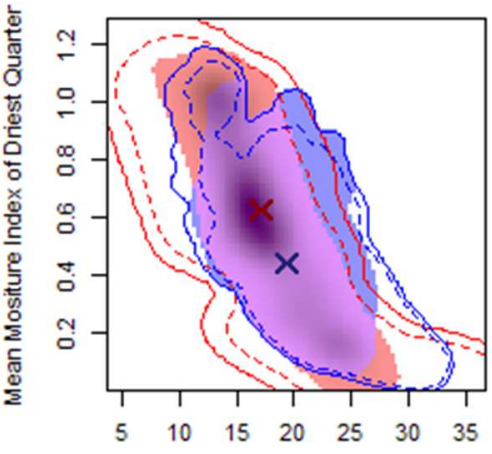

Mean Temperature of Warmest Quarter

Introduced Climate Densities

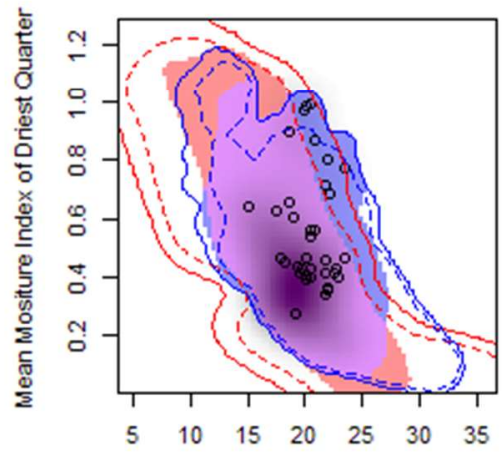

Mean Temperature of Warmest Quarter

Figure S15. Introduction of Geranium molle to Australia

Table S19. Niche shift indices for the introduction of Geranium molle to Australia

\begin{tabular}{cccccc}
\hline \multirow{2}{*}{$\begin{array}{c}\text { Marginal } \\
\text { climate }\end{array}$} & \multicolumn{2}{c}{ Niche Expansion } & & \multicolumn{2}{c}{ Niche Unfilling } \\
\cline { 2 - 3 } \cline { 5 - 6 } threshold (\%) & $\begin{array}{c}\text { Analog } \\
\text { climate }\end{array}$ & $\begin{array}{c}\text { Non-analog } \\
\text { climate }\end{array}$ & & $\begin{array}{c}\text { Analog } \\
\text { climate }\end{array}$ & $\begin{array}{c}\text { Non-analog } \\
\text { climate }\end{array}$ \\
\hline 99 & 0.061 & 0.067 & & 0.084 & 0.100 \\
95 & 0.052 & 0.064 & & 0.084 & 0.112 \\
90 & 0.043 & 0.059 & & 0.083 & 0.121 \\
85 & 0.032 & 0.055 & & 0.080 & 0.132 \\
80 & 0.018 & 0.049 & & 0.077 & 0.148 \\
75 & 0.008 & 0.044 & & 0.070 & 0.171 \\
\hline
\end{tabular}




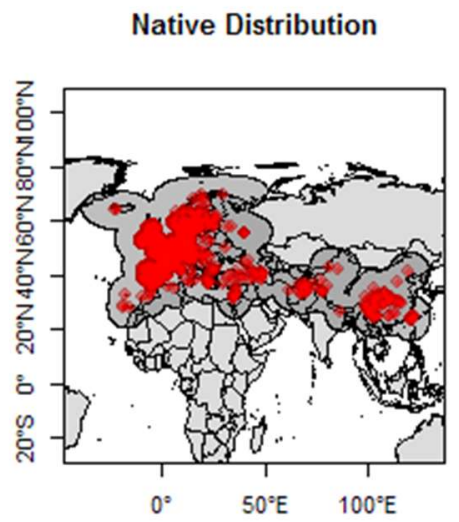

Introduced Distribution

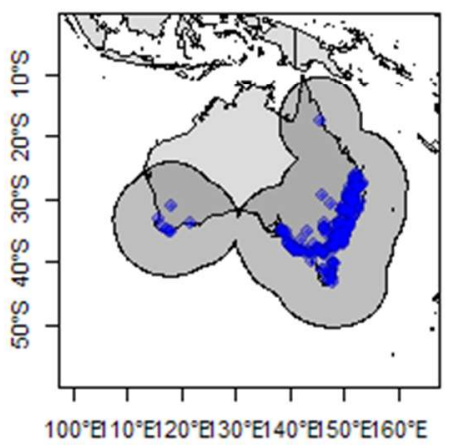

Native Climate Space

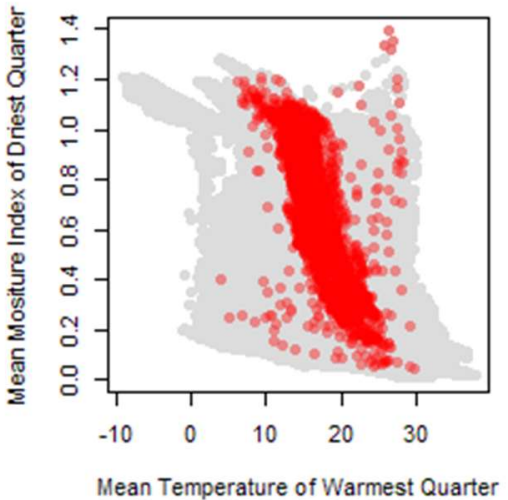

Introduced Climate Space

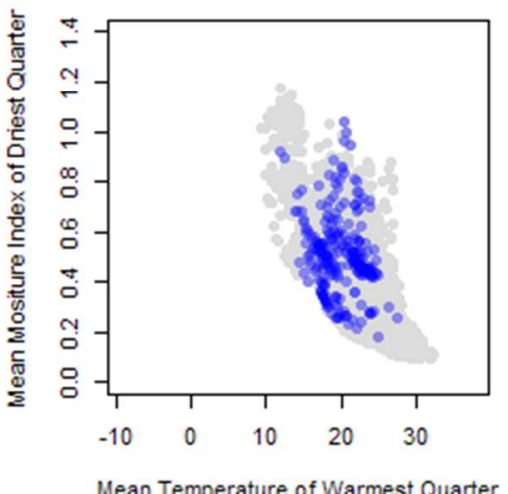

Native Climate Densities

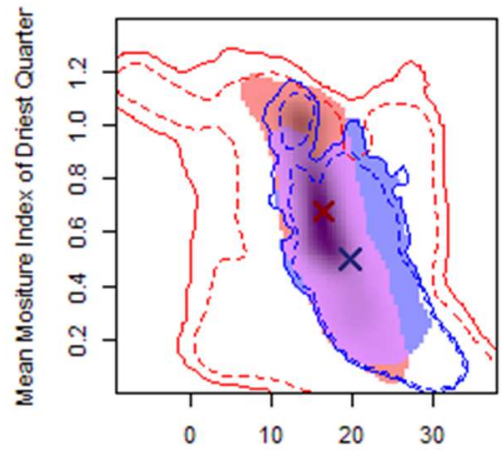

Mean Temperature of Warmest Quarter

Introduced Climate Densities

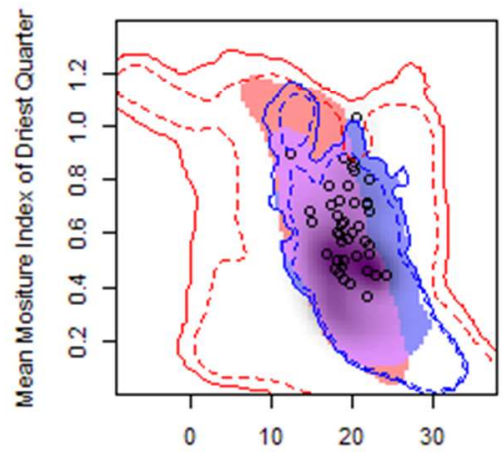

Mean Temperature of Warmest Quarter

Figure S16. Introduction of Medicago Iupulina to Australia

Table S20. Niche shift indices for the introduction of Medicago lupulina to Australia

\begin{tabular}{cccccc}
\hline \multirow{2}{*}{$\begin{array}{c}\text { Marginal } \\
\text { climate } \\
\text { threshold }\end{array}$} & \multicolumn{2}{c}{$\begin{array}{c}\text { Niche Expansion } \\
\text { climate }\end{array}$} & $\begin{array}{c}\text { Non-analog } \\
\text { climate }\end{array}$ & & \multicolumn{2}{c}{$\begin{array}{c}\text { Analog } \\
\text { climate }\end{array}$} & $\begin{array}{c}\text { Non-analog } \\
\text { climate }\end{array}$ \\
\cline { 2 - 3 } & 0.128 & 0.128 & & 0.106 & 0.143 \\
99 & 0.126 & 0.126 & & 0.102 & 0.150 \\
95 & 0.123 & 0.123 & & 0.099 & 0.162 \\
90 & 0.120 & 0.120 & & 0.094 & 0.178 \\
85 & 0.116 & 0.116 & & 0.088 & 0.206 \\
80 & 0.112 & 0.113 & & 0.083 & 0.239 \\
75 & & & & & \\
\hline
\end{tabular}


Native Distribution

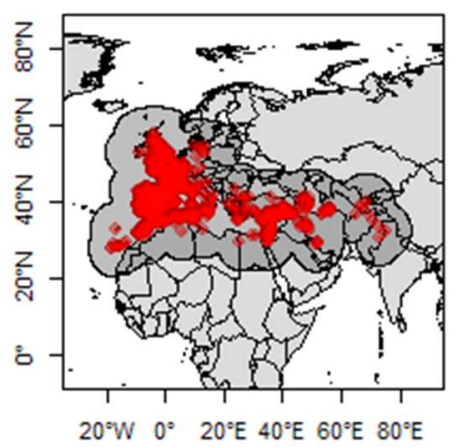

Introduced Distribution

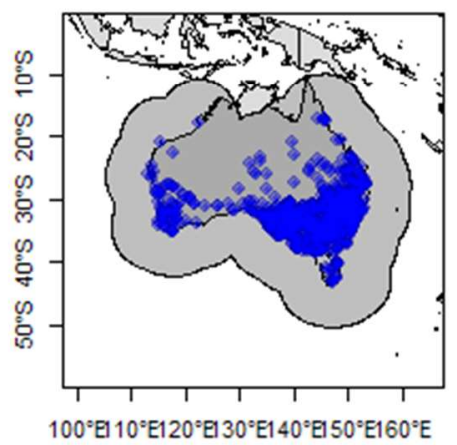

Native Climate Space

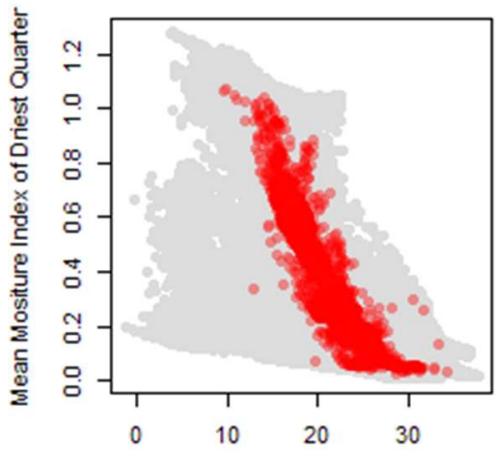

Mean Temperature of Warmest Quarter

Introduced Climate Space

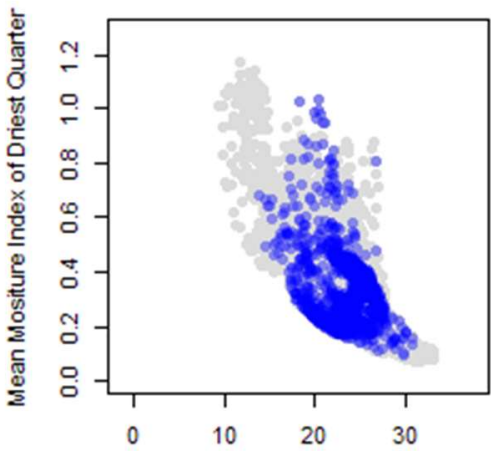

Mean Temperature of Warmest Quarter
Native Climate Densities

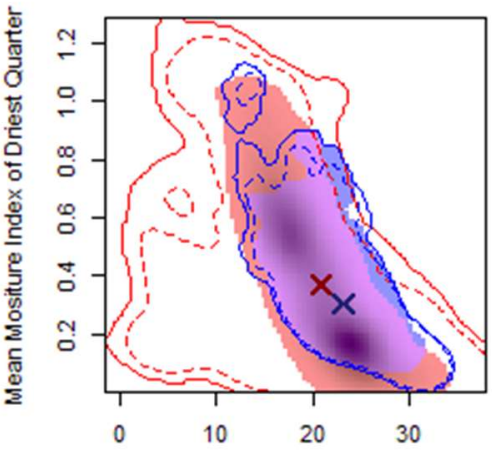

Mean Temperature of Warmest Quarter

Introduced Climate Densities

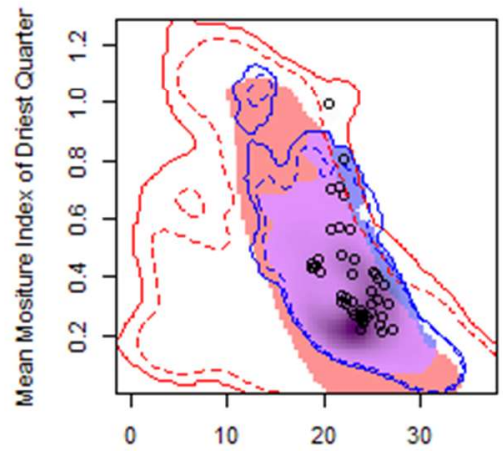

Mean Temperature of Warmest Quarter

Figure S17. Introduction of Medicago polymorpha to Australia

Table S21. Niche shift indices for the introduction of Medicago polymorpha to Australia

\begin{tabular}{|c|c|c|c|c|}
\hline \multirow{2}{*}{$\begin{array}{l}\text { Marginal } \\
\text { climate } \\
\text { threshold }\end{array}$} & \multicolumn{2}{|c|}{ Niche Expansion } & \multicolumn{2}{|c|}{ Niche Unfilling } \\
\hline & $\begin{array}{l}\text { Analog } \\
\text { climate }\end{array}$ & $\begin{array}{l}\text { Non-analog } \\
\text { climate }\end{array}$ & $\begin{array}{l}\text { Analog } \\
\text { climate }\end{array}$ & $\begin{array}{l}\text { Non-analog } \\
\text { climate }\end{array}$ \\
\hline 99 & 0.043 & 0.043 & 0.105 & 0.186 \\
\hline 95 & 0.041 & 0.042 & 0.097 & 0.187 \\
\hline 90 & 0.040 & 0.042 & 0.088 & 0.191 \\
\hline 85 & 0.037 & 0.041 & 0.076 & 0.195 \\
\hline 80 & 0.034 & 0.039 & 0.064 & 0.198 \\
\hline 75 & 0.030 & 0.037 & 0.050 & 0.202 \\
\hline
\end{tabular}




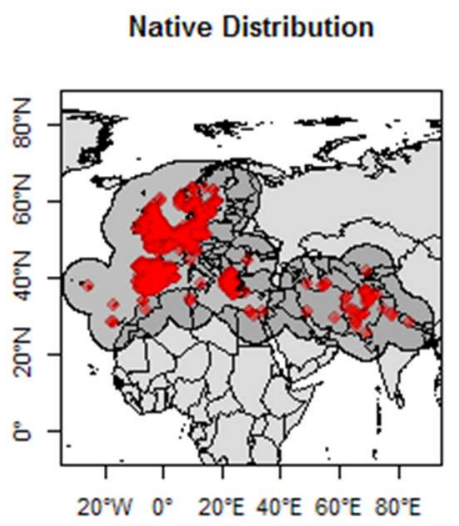

Introduced Distribution

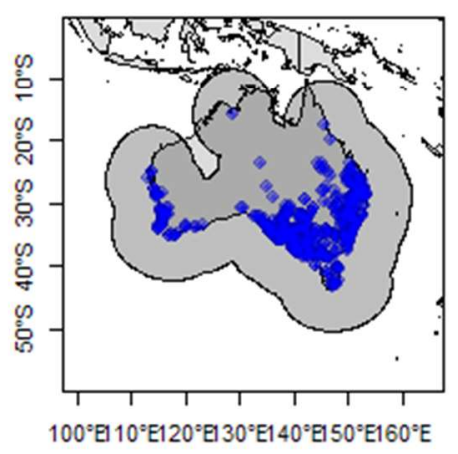

Figure S18. Introduction of Melilotus indicus to Australia
Native Climate Space

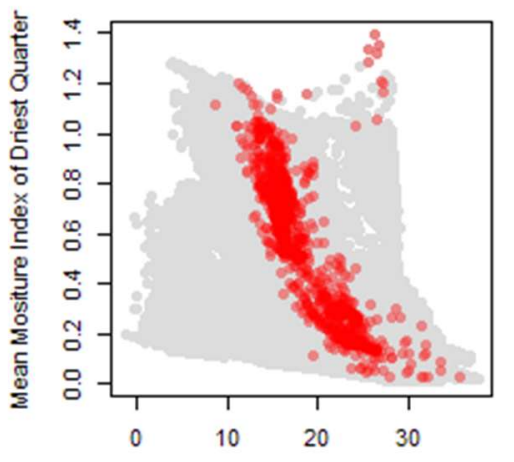

Mean Temperature of Warmest Quarter

Introduced Climate Space

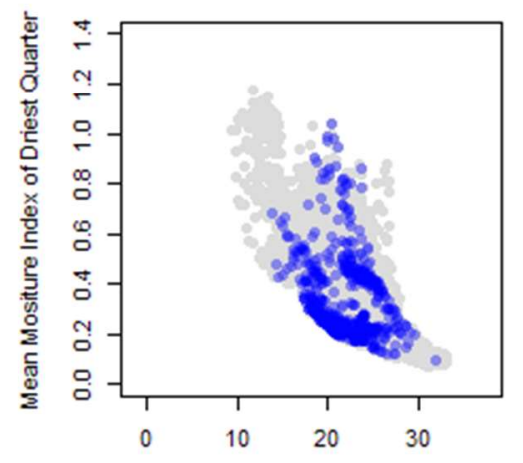

Mean Temperature of Warmest Quarter

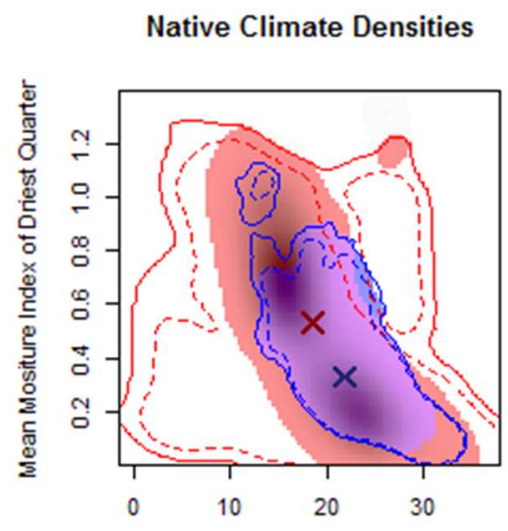

Mean Temperature of Warmest Quarter

Introduced Climate Densities

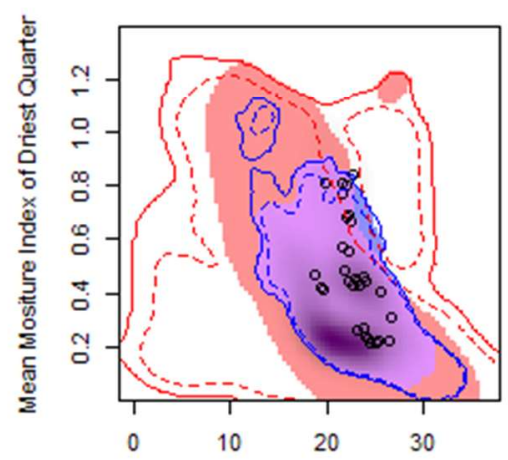

Mean Temperature of Warmest Quarter

Table S22. Niche shift indices for the introduction of Melilotus indicus to Australia

\begin{tabular}{cccccc}
\hline \multirow{2}{*}{$\begin{array}{c}\text { Marginal } \\
\text { climate }\end{array}$} & \multicolumn{2}{c}{ Niche Expansion } & & \multicolumn{2}{c}{ Niche Unfilling } \\
\cline { 2 - 3 } \cline { 5 - 6 } threshold $(\%)$ & $\begin{array}{c}\text { Analog } \\
\text { climate }\end{array}$ & $\begin{array}{c}\text { Non-analog } \\
\text { climate }\end{array}$ & & $\begin{array}{c}\text { Analog } \\
\text { climate }\end{array}$ & $\begin{array}{c}\text { Non-analog } \\
\text { climate }\end{array}$ \\
\hline 99 & 0.118 & 0.173 & & 0.007 & 0.385 \\
95 & 0.097 & 0.179 & & 0.007 & 0.391 \\
90 & 0.060 & 0.190 & & 0.007 & 0.397 \\
85 & 0.018 & 0.226 & & 0.007 & 0.406 \\
80 & 0.003 & 0.277 & & 0.007 & 0.414 \\
75 & 0 & 0.309 & & 0.006 & 0.423 \\
\hline
\end{tabular}




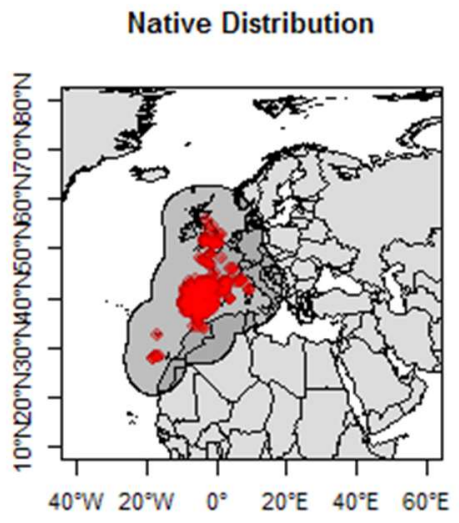

Introduced Distribution

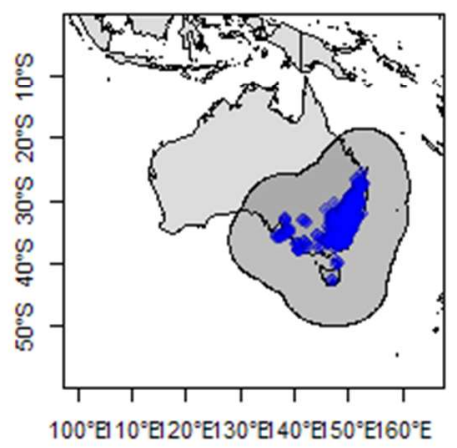

Native Climate Space

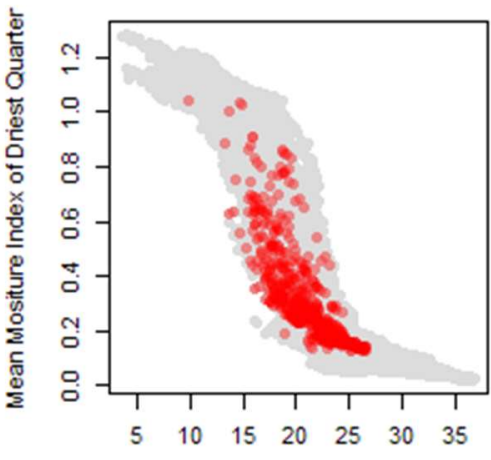

Mean Temperature of Warmest Quarter

Introduced Climate Space

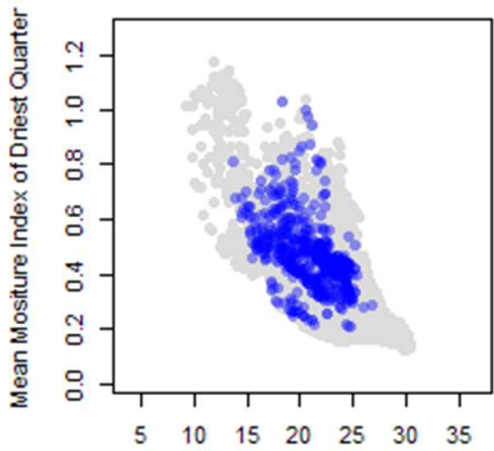

Mean Temperature of Warmest Quarter
Native Climate Densities

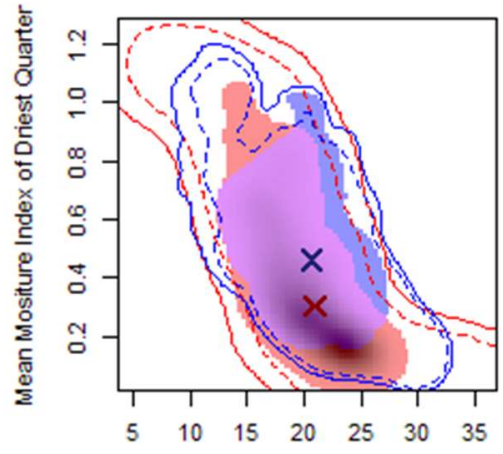

Mean Temperature of Warmest Quarter

Introduced Climate Densities

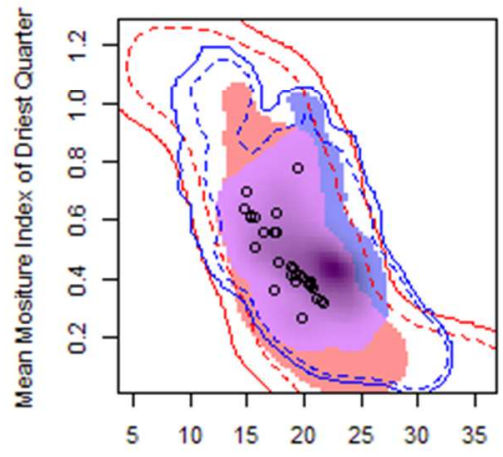

Mean Temperature of Warmest Quarter

Figure S19. Introduction of Petrorhagia nanteullii to Australia

Table S23. Niche shift indices for the introduction of Petrorhagia nanteullii to Australia

\begin{tabular}{cccccc}
\hline \multirow{2}{*}{$\begin{array}{c}\text { Marginal } \\
\text { climate }\end{array}$} & \multicolumn{2}{c}{ Niche Expansion } & & \multicolumn{2}{c}{ Niche Unfilling } \\
\cline { 2 - 3 } \cline { 5 - 6 } threshold $(\%)$ & $\begin{array}{c}\text { Analog } \\
\text { climate }\end{array}$ & $\begin{array}{c}\text { Non-analog } \\
\text { climate }\end{array}$ & & $\begin{array}{c}\text { Analog } \\
\text { climate }\end{array}$ & $\begin{array}{c}\text { Non-analog } \\
\text { climate }\end{array}$ \\
\hline 99 & 0.068 & 0.068 & & 0.227 & 0.235 \\
95 & 0.068 & 0.067 & & 0.225 & 0.235 \\
90 & 0.067 & 0.066 & & 0.222 & 0.235 \\
85 & 0.066 & 0.067 & & 0.218 & 0.235 \\
80 & 0.062 & 0.066 & & 0.213 & 0.235 \\
75 & 0.055 & 0.065 & & 0.209 & 0.235 \\
\hline
\end{tabular}




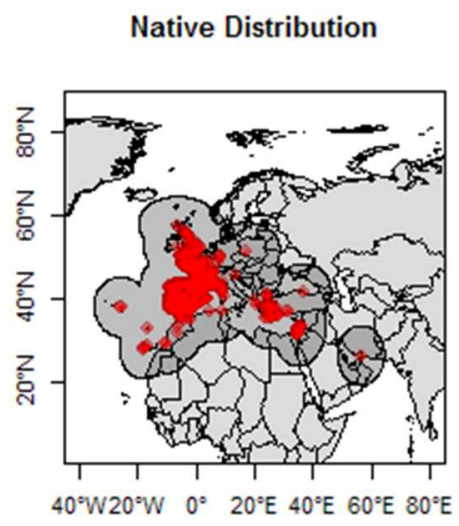

Introduced Distribution

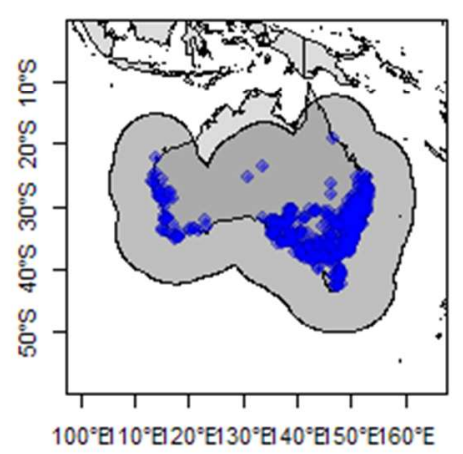

Native Climate Space

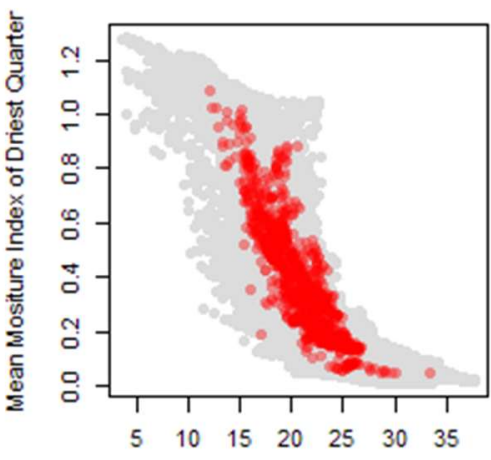

Mean Temperature of Warmest Quarter

Introduced Climate Space

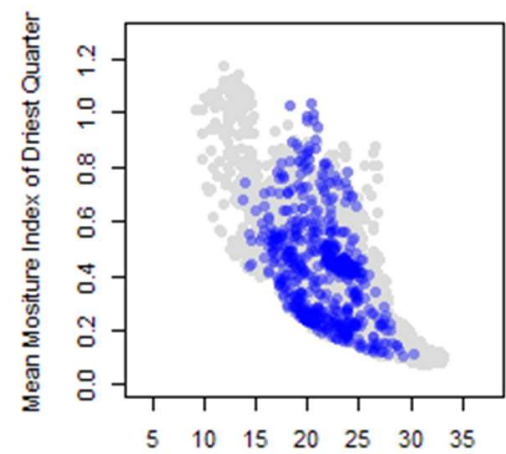

Mean Temperature of Warmest Quarter
Native Climate Densities

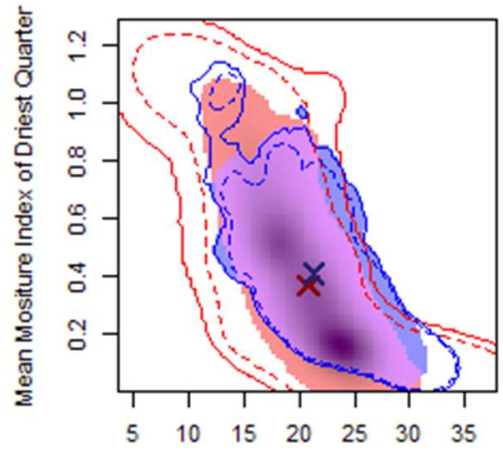

Mean Temperature of Warmest Quarter

Introduced Climate Densities

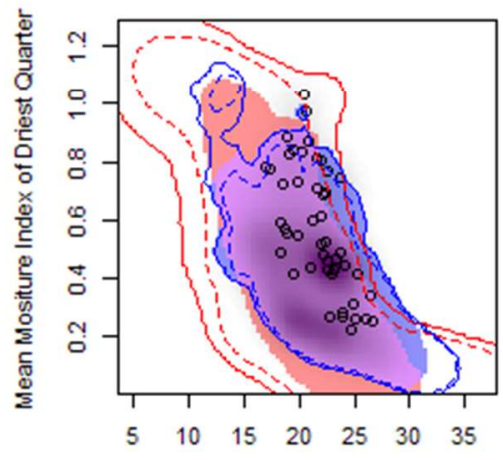

Mean Temperature of Warmest Quarter

Figure S20. Introduction of Polycarpon tetraphyllum to Australia

Table S24. Niche shift indices for the introduction of Polycarpon tetraphyllum to Australia

\begin{tabular}{cccccc}
\hline $\begin{array}{c}\text { Marginal } \\
\text { climate } \\
\text { threshold } \\
(\%)\end{array}$ & $\begin{array}{c}\text { Analog } \\
\text { climate }\end{array}$ & $\begin{array}{c}\text { Non-analog } \\
\text { climate }\end{array}$ & & & \multicolumn{2}{c}{$\begin{array}{c}\text { Nnalog } \\
\text { climate }\end{array}$} & $\begin{array}{c}\text { Non-analog } \\
\text { climate }\end{array}$ \\
\cline { 2 - 3 } & 0.051 & 0.061 & & 0.013 & 0.070 \\
95 & 0.043 & 0.058 & & 0.011 & 0.076 \\
90 & 0.036 & 0.055 & & 0.008 & 0.083 \\
85 & 0.028 & 0.053 & & 0.006 & 0.090 \\
80 & 0.020 & 0.051 & & 0.003 & 0.100 \\
75 & 0.011 & 0.050 & & 0.002 & 0.112 \\
\hline
\end{tabular}




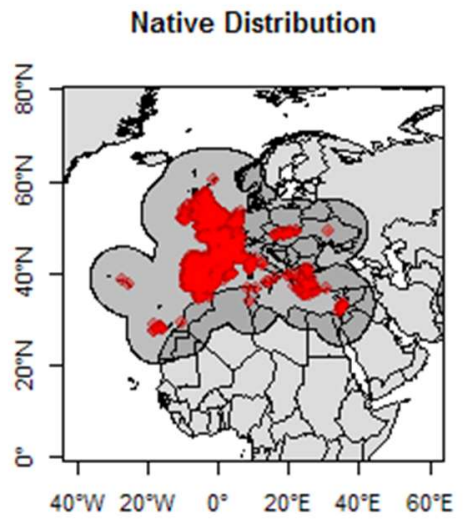

Introduced Distribution

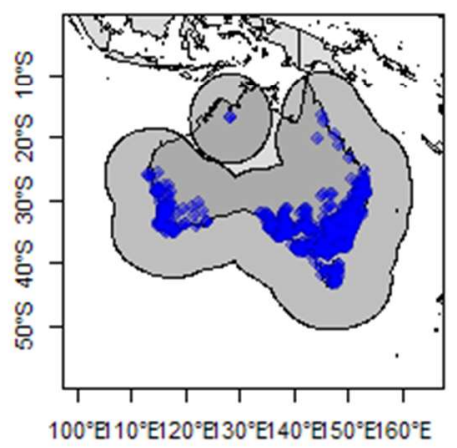

Native Climate Space

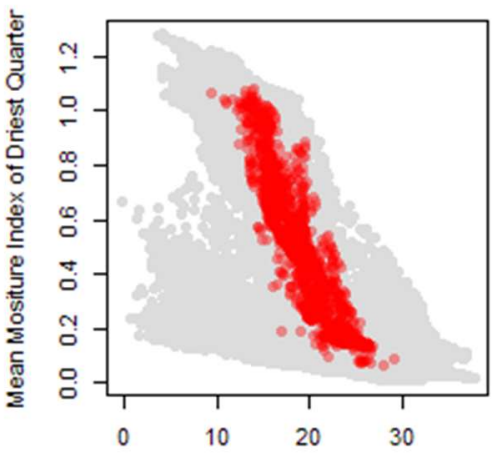

Mean Temperature of Warmest Quarter

Introduced Climate Space

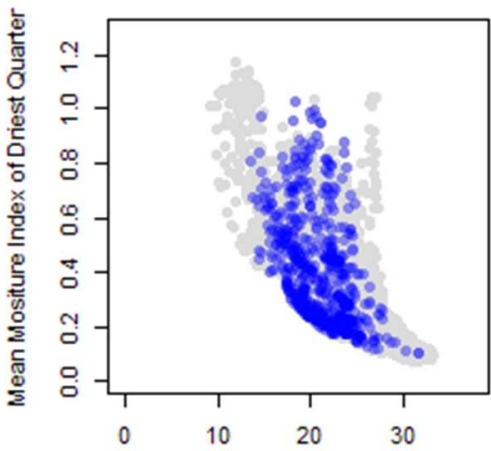

Mean Temperature of Warmest Quarter
Native Climate Densities

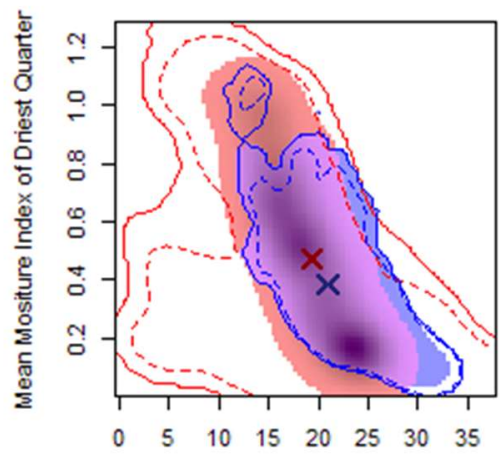

Mean Temperature of Warmest Quarter

Introduced Climate Densities

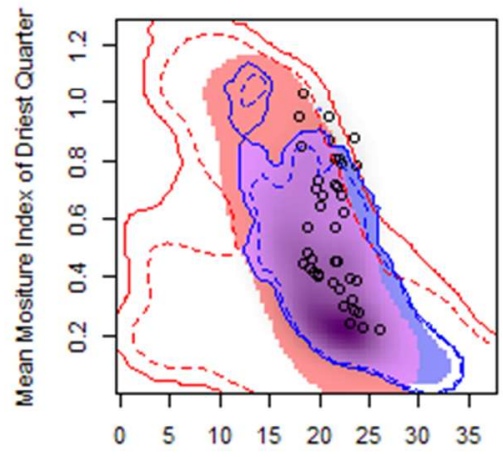

Mean Temperature of Warmest Quarter

Figure S21. Introduction of Silene gallica to Australia

Table S25. Niche shift indices for the introduction of Silene gallica to Australia

\begin{tabular}{cccccc}
\hline \multirow{2}{*}{$\begin{array}{c}\text { Marginal } \\
\text { climate } \\
\text { threshold }(\%)\end{array}$} & \multicolumn{2}{c}{ Niche Expansion } & & \multicolumn{2}{c}{ Niche Unfilling } \\
\cline { 2 - 3 } \cline { 5 - 6 } & $\begin{array}{c}\text { Analog } \\
\text { climate }\end{array}$ & $\begin{array}{c}\text { Non-analog } \\
\text { climate }\end{array}$ & & $\begin{array}{c}\text { Analog } \\
\text { climate }\end{array}$ & Non-analog climate \\
\hline 99 & 0.234 & 0.329 & & 0 & 0.458 \\
95 & 0.211 & 0.328 & & 0 & 0.473 \\
90 & 0.188 & 0.326 & & 0 & 0.489 \\
85 & 0.156 & 0.324 & & 0 & 0.503 \\
80 & 0.124 & 0.322 & & 0 & 0.521 \\
75 & 0.093 & 0.324 & & 0 & 0.539 \\
\hline
\end{tabular}




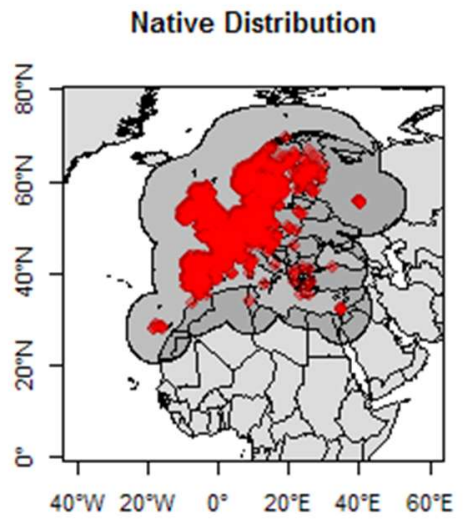

Introduced Distribution

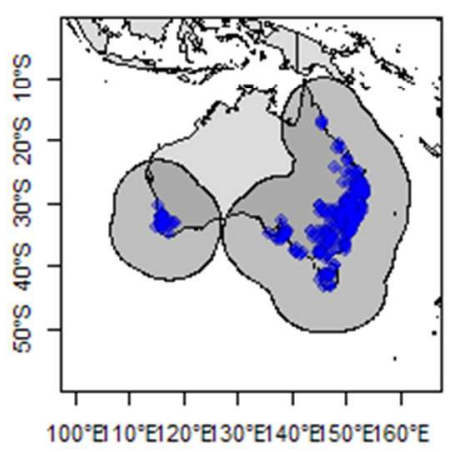

Figure S22. Introduction of Stachys arvensis to Australia
Native Climate Space

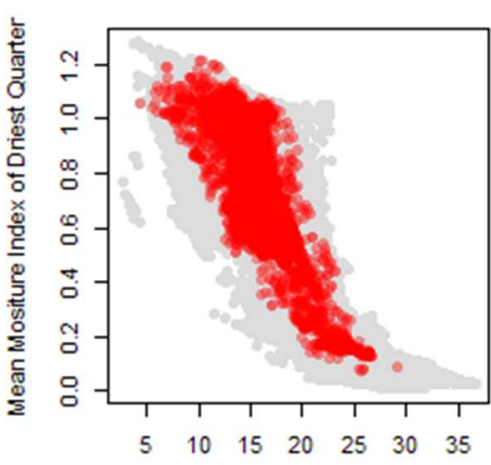

Mean Temperature of Warmest Quarter

Introduced Climate Space

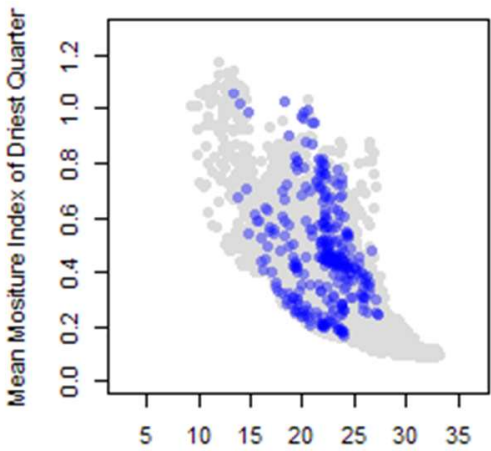

Mean Temperature of Warmest Quarter

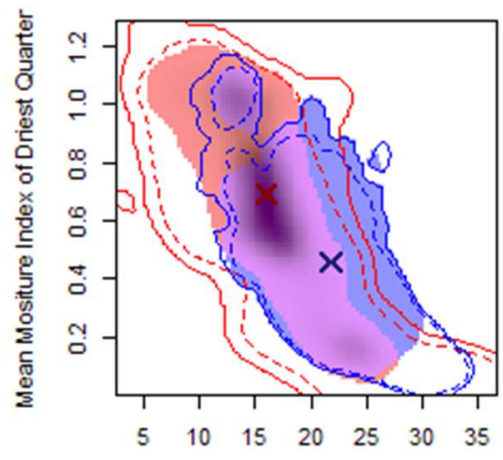

Mean Temperature of Warmest Quarter

Introduced Climate Densities

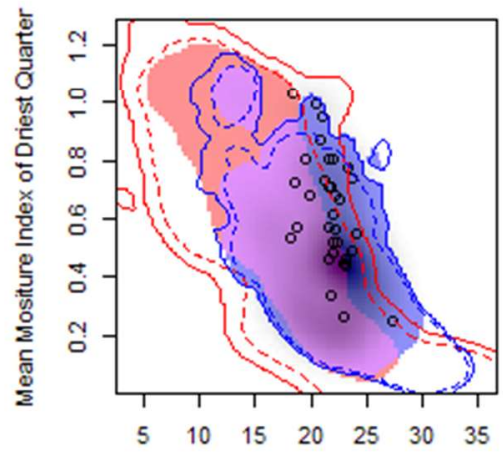

Mean Temperature of Warmest Quarter

Table S26. Niche shift indices for the introduction of Stachys arvensis to Australia

\begin{tabular}{cccccc}
\hline \multirow{2}{*}{$\begin{array}{c}\text { Marginal } \\
\text { climate }\end{array}$} & \multicolumn{2}{c}{ Niche Expansion } & & \multicolumn{2}{c}{ Niche Unfilling } \\
\cline { 2 - 3 } \cline { 5 - 6 } threshold $(\%)$ & $\begin{array}{c}\text { Analog } \\
\text { climate }\end{array}$ & $\begin{array}{c}\text { Non-analog } \\
\text { climate }\end{array}$ & & $\begin{array}{c}\text { Analog } \\
\text { climate }\end{array}$ & $\begin{array}{c}\text { Non-analog } \\
\text { climate }\end{array}$ \\
\hline 99 & 0.268 & 0.326 & & 0.009 & 0.095 \\
95 & 0.252 & 0.325 & & 0.008 & 0.098 \\
90 & 0.230 & 0.323 & & 0.007 & 0.101 \\
85 & 0.189 & 0.320 & & 0.005 & 0.106 \\
80 & 0.131 & 0.318 & & 0.004 & 0.114 \\
75 & 0.060 & 0.314 & & 0.003 & 0.123 \\
\hline
\end{tabular}




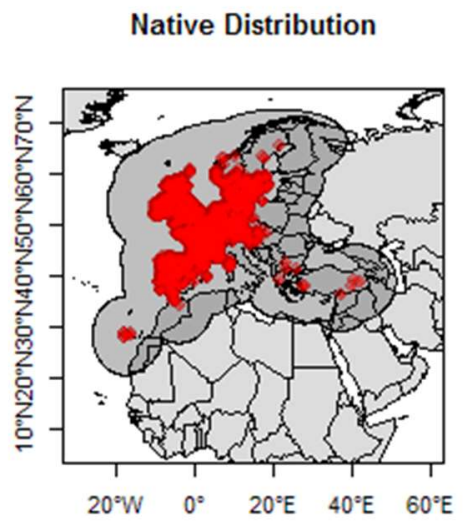

Introduced Distribution

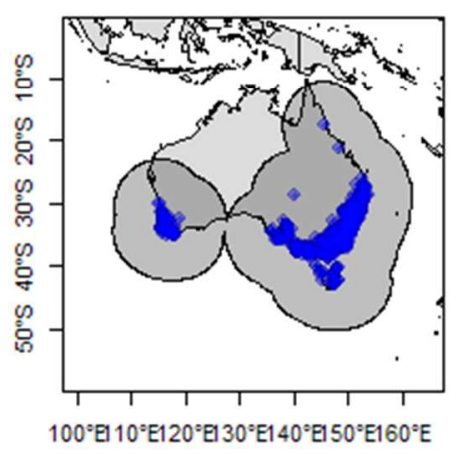

Native Climate Space

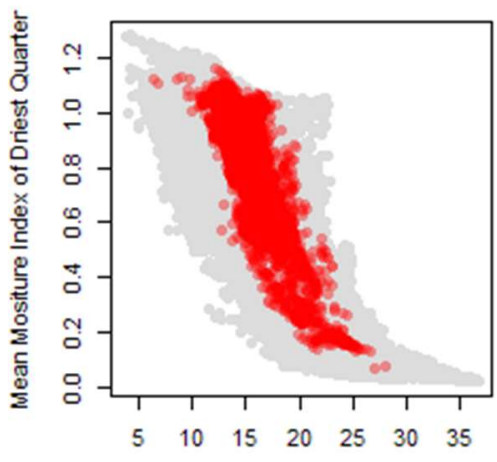

Mean Temperature of Warmest Quarter

Introduced Climate Space

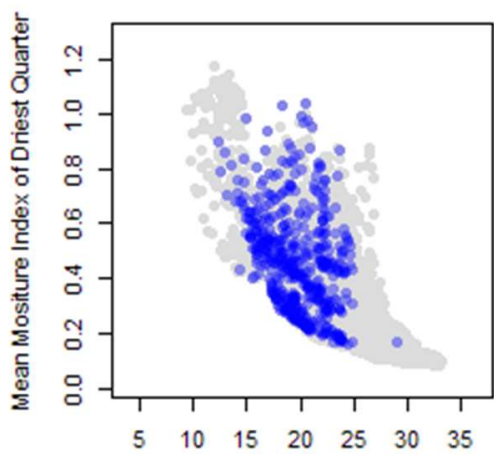

Mean Temperature of Warmest Quarter
Native Climate Densities

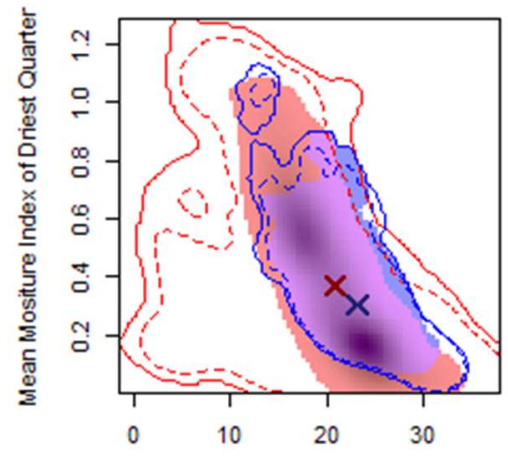

Mean Temperature of Warmest Quarter

Introduced Climate Densities

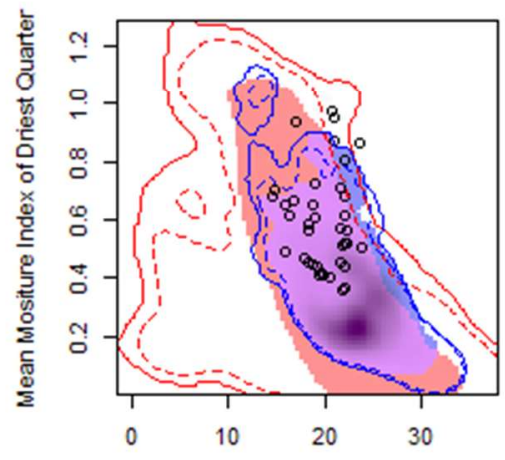

Mean Temperature of Warmest Quarter

Figure S23. Introduction of Trifolium dubium to Australia

Table S27. Niche shift indices for the introduction of Trifolium dubium to Australia

\begin{tabular}{cccccc}
\hline \multirow{2}{*}{$\begin{array}{c}\text { Marginal } \\
\text { climate }\end{array}$} & \multicolumn{2}{c}{ Niche Expansion } & & \multicolumn{2}{c}{ Niche Unfilling } \\
\cline { 2 - 3 } \cline { 5 - 6 } threshold (\%) & $\begin{array}{c}\text { Analog } \\
\text { climate }\end{array}$ & $\begin{array}{c}\text { Non-analog } \\
\text { climate }\end{array}$ & & $\begin{array}{c}\text { Analog } \\
\text { climate }\end{array}$ & $\begin{array}{c}\text { Non-analog } \\
\text { climate }\end{array}$ \\
\hline 99 & 0.304 & 0.387 & & 0.003 & 0.070 \\
95 & 0.288 & 0.386 & & 0.001 & 0.073 \\
90 & 0.269 & 0.385 & & 0 & 0.078 \\
85 & 0.246 & 0.383 & & 0 & 0.084 \\
80 & 0.207 & 0.380 & & 0 & 0.093 \\
75 & 0.158 & 0.376 & & 0 & 0.106 \\
\hline
\end{tabular}




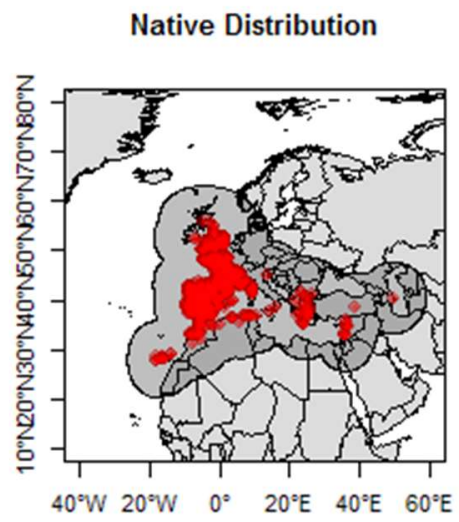

Introduced Distribution

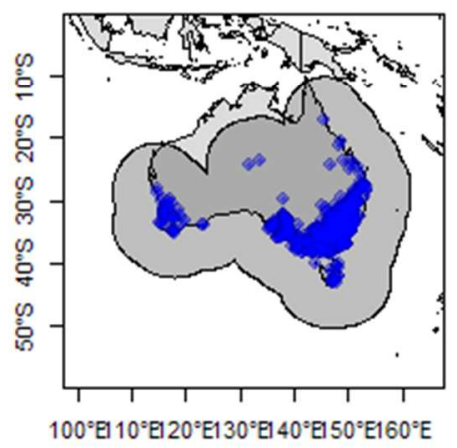

Native Climate Space

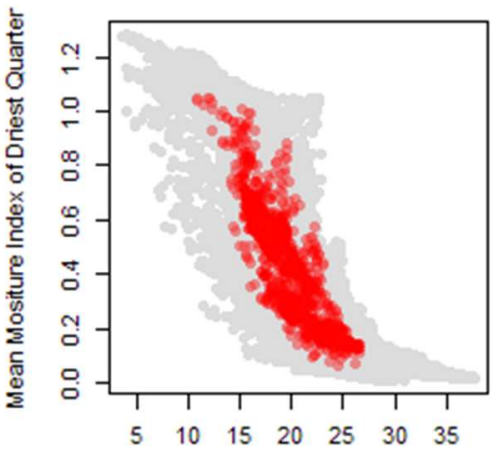

Mean Temperature of Warmest Quarter

Introduced Climate Space

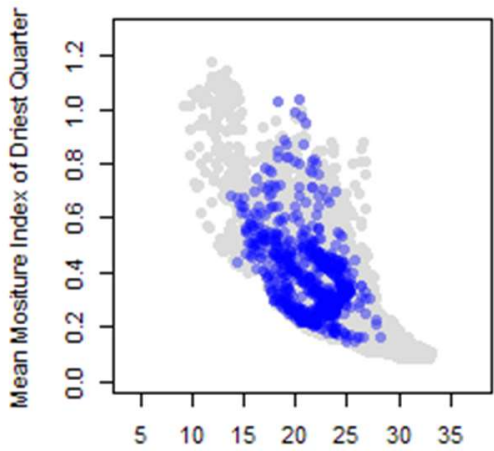

Mean Temperature of Warmest Quarter
Native Climate Densities

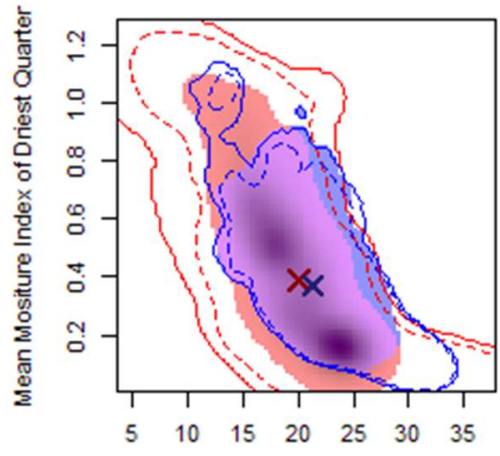

Mean Temperature of Warmest Quarter

Introduced Climate Densities

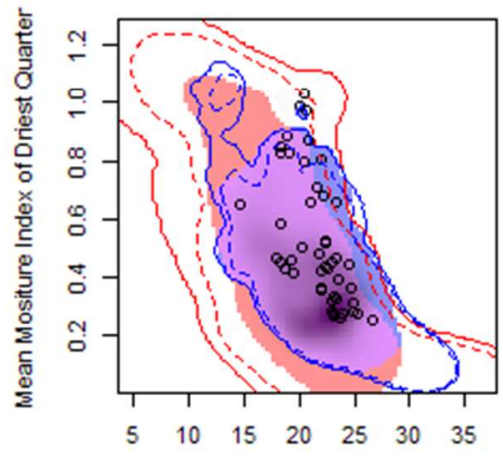

Mean Temperature of Warmest Quarter

Figure S24. Introduction of Trifolium glomeratum to Australia

Table S28. Niche shift indices for the introduction of Trifolium glomeratum to Australia

\begin{tabular}{cccccc}
\hline \multirow{2}{*}{$\begin{array}{c}\text { Marginal } \\
\text { climate }\end{array}$} & \multicolumn{2}{c}{ Niche Expansion } & & \multicolumn{2}{c}{ Niche Unfilling } \\
\cline { 2 - 3 } \cline { 5 - 6 } threshold (\%) & $\begin{array}{c}\text { Analog } \\
\text { climate }\end{array}$ & $\begin{array}{c}\text { Non-analog } \\
\text { climate }\end{array}$ & & $\begin{array}{c}\text { Analog } \\
\text { climate }\end{array}$ & $\begin{array}{c}\text { Non-analog } \\
\text { climate }\end{array}$ \\
\hline 99 & 0.006 & 0.007 & & 0.018 & 0.080 \\
95 & 0.004 & 0.006 & & 0.015 & 0.085 \\
90 & 0.003 & 0.006 & & 0.011 & 0.091 \\
85 & 0.001 & 0.006 & & 0.008 & 0.100 \\
80 & 0 & 0.007 & & 0.005 & 0.111 \\
75 & 0 & 0.009 & & 0.003 & 0.124 \\
\hline
\end{tabular}




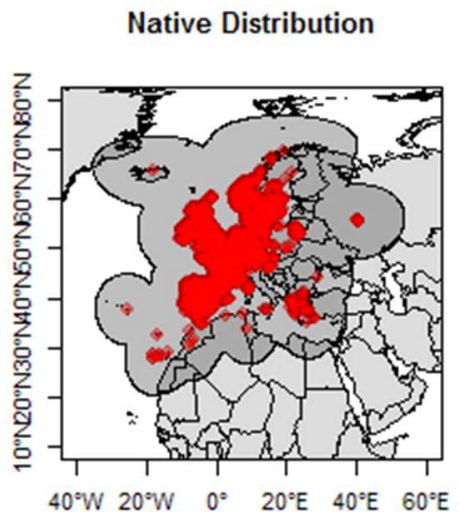

Introduced Distribution

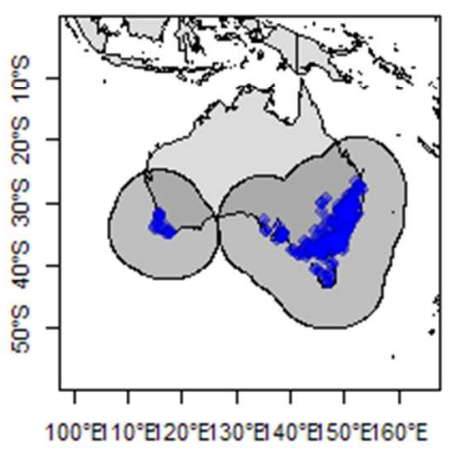

Native Climate Space

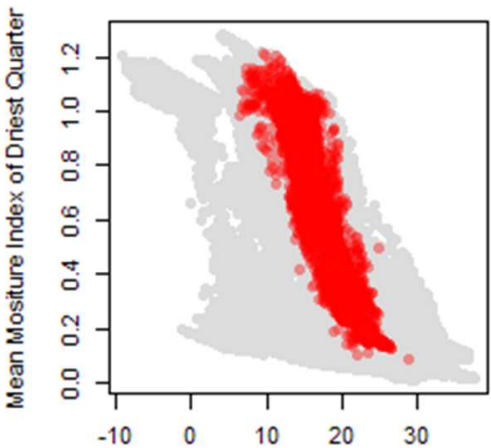

Mean Temperature of Warmest Quarter

Introduced Climate Space

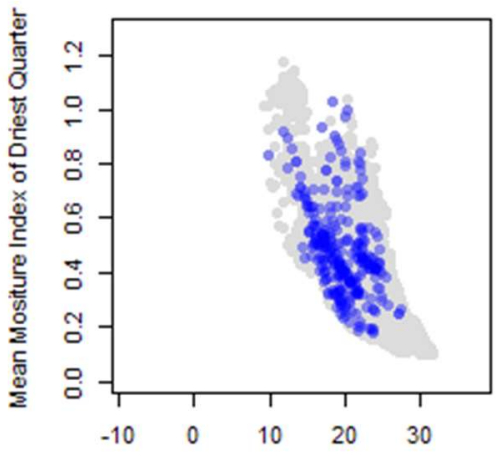

Mean Temperature of Warmest Quarter
Native Climate Densities

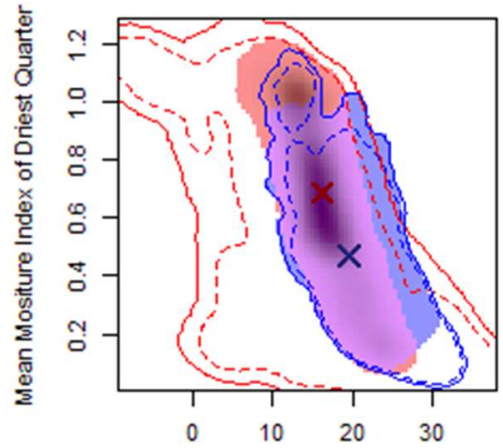

Mean Temperature of Warmest Quarter

Introduced Climate Densities

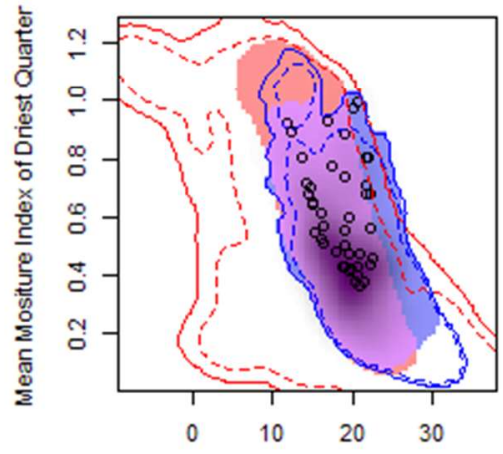

Mean Temperature of Warmest Quarte

Figure S25. Introduction of Veronica arvensis to Australia

Table S29. Niche shift indices for the introduction of Veronica arvensis to Australia

\begin{tabular}{cccccc}
\hline \multirow{2}{*}{$\begin{array}{c}\text { Marginal } \\
\text { climate }\end{array}$} & \multicolumn{2}{c}{ Niche Expansion } & & \multicolumn{2}{c}{ Niche Unfilling } \\
\cline { 2 - 3 } \cline { 5 - 6 } threshold $(\%)$ & $\begin{array}{c}\text { Analog } \\
\text { climate }\end{array}$ & $\begin{array}{c}\text { Non-analog } \\
\text { climate }\end{array}$ & & $\begin{array}{c}\text { Analog } \\
\text { climate }\end{array}$ & $\begin{array}{c}\text { Non-analog } \\
\text { climate }\end{array}$ \\
\hline 99 & 0.093 & 0.096 & & 0.110 & 0.132 \\
95 & 0.088 & 0.095 & & 0.110 & 0.146 \\
90 & 0.081 & 0.092 & & 0.108 & 0.160 \\
85 & 0.073 & 0.090 & & 0.106 & 0.178 \\
80 & 0.063 & 0.087 & & 0.104 & 0.201 \\
75 & 0.051 & 0.085 & & 0.099 & 0.235 \\
\hline
\end{tabular}




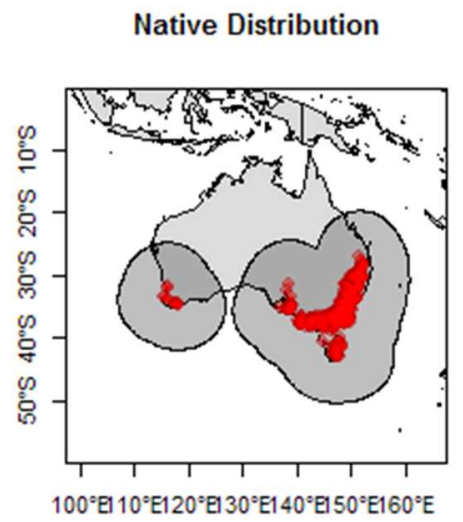

Introduced Distribution

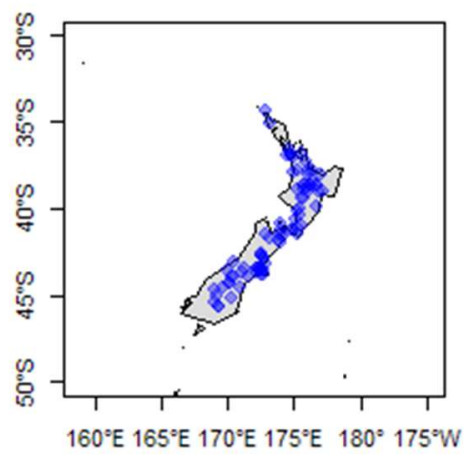

Native Climate Space

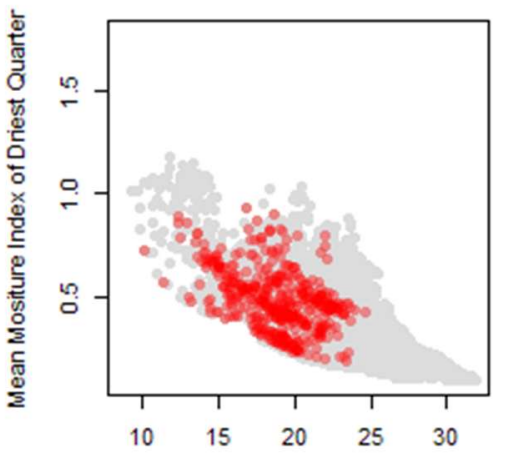

Mean Temperature of Warmest Quarter Introduced Climate Space

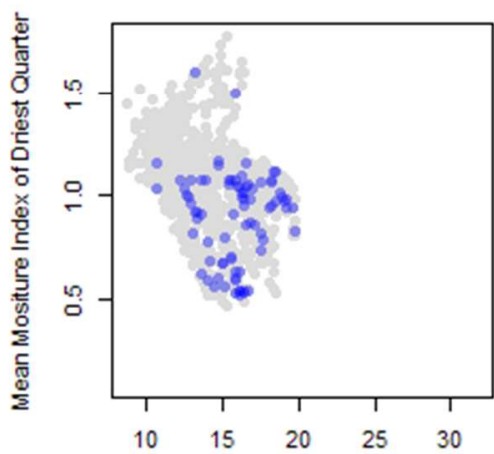

Mean Temperature of Warmest Quarter
Native Climate Densities

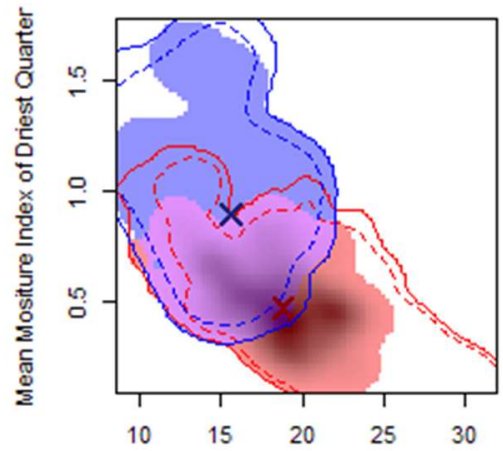

Mean Temperature of Warmest Quarter

Introduced Climate Densities

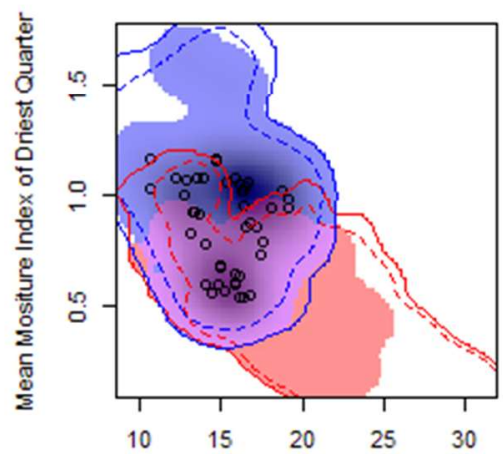

Mean Temperature of Warmest Quarter

Figure S26. Introduction of Acaena agnipilia to New Zealand

Table S30. Niche shift indices for the introduction of Acaena agnipilia to New Zealand

\begin{tabular}{cccccc}
\hline $\begin{array}{c}\text { Marginal } \\
\text { climate } \\
\text { threshold }\end{array}$ & \multicolumn{2}{c}{ Niche Expansion } & & \multicolumn{2}{c}{ Niche Unfilling } \\
\cline { 2 - 3 } \cline { 5 - 6 } & $\begin{array}{c}\text { Analog } \\
\text { climate }\end{array}$ & $\begin{array}{c}\text { Non-analog } \\
\text { climate }\end{array}$ & & $\begin{array}{c}\text { Analog } \\
\text { climate }\end{array}$ & $\begin{array}{c}\text { Non-analog } \\
\text { climate }\end{array}$ \\
\hline 99 & 0.302 & 0.482 & & 0.071 & 0.500 \\
95 & 0.277 & 0.497 & & 0.037 & 0.520 \\
90 & 0.272 & 0.516 & & 0.005 & 0.541 \\
85 & 0.254 & 0.535 & & 0 & 0.576 \\
80 & 0.236 & 0.556 & & 0 & 0.616 \\
75 & 0.211 & 0.585 & & 0 & 0.653 \\
\hline
\end{tabular}




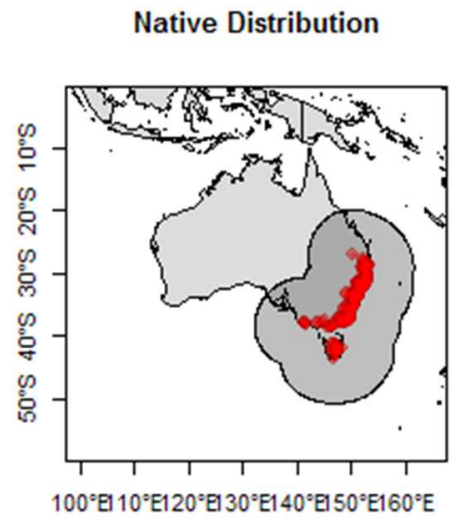

Introduced Distribution

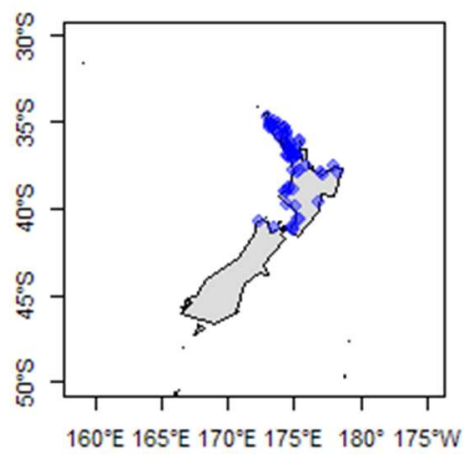

Native Climate Space

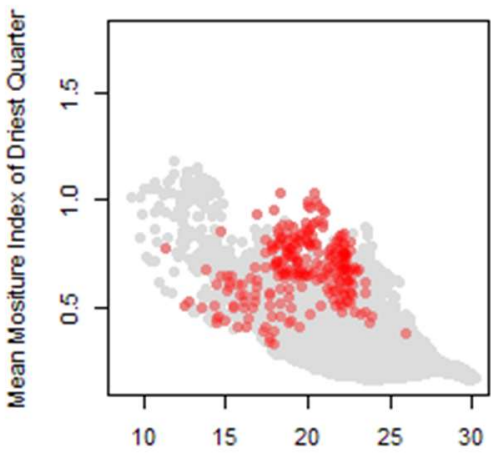

Mean Temperature of Warmest Quarter Introduced Climate Space

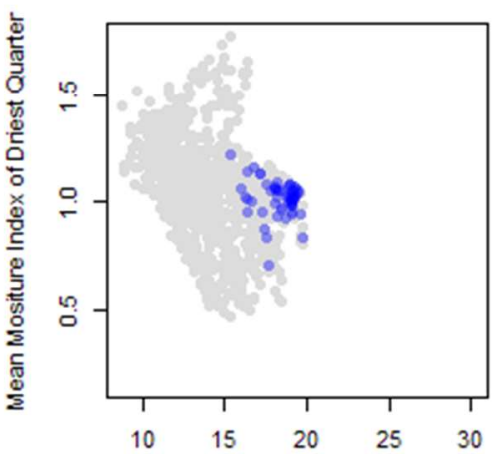

Mean Temperature of Warmest Quarter
Native Climate Densities

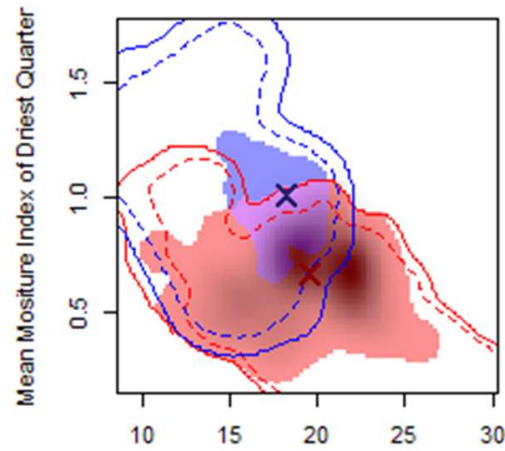

Mean Temperature of Warmest Quarter

Introduced Climate Densities

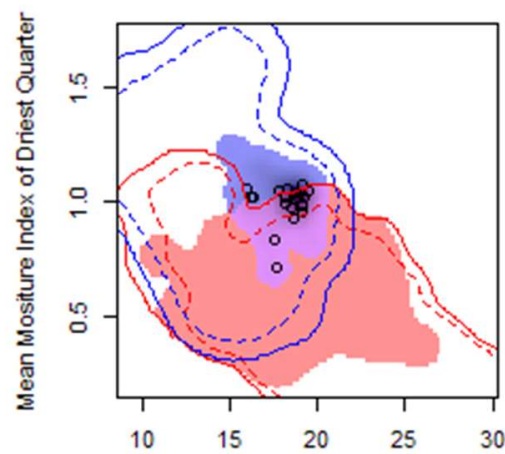

Mean Temperature of Warmest Quarter

Figure S27. Introduction of Carex longebrachiata to New Zealand

Table S31. Niche shift indices for the introduction of Carex longebrachiata to New Zealand

\begin{tabular}{cccccc}
\hline \multirow{2}{*}{$\begin{array}{c}\text { Marginal } \\
\text { climate } \\
\text { threshold }\end{array}$} & \multicolumn{2}{c}{ Niche Expansion } & & \multicolumn{2}{c}{ Niche Unfilling } \\
\cline { 2 - 3 } \cline { 5 - 6 } & $\begin{array}{c}\text { Analog } \\
\text { climate }\end{array}$ & $\begin{array}{c}\text { Non-analog } \\
\text { climate }\end{array}$ & & $\begin{array}{c}\text { Analog } \\
\text { climate }\end{array}$ & $\begin{array}{c}\text { Non-analog } \\
\text { climate }\end{array}$ \\
\hline 99 & 0.034 & 0.363 & & 0.675 & 0.778 \\
95 & 0.031 & 0.503 & & 0.661 & 0.780 \\
90 & 0.027 & 0.641 & & 0.640 & 0.782 \\
85 & 0.026 & 0.711 & & 0.617 & 0.784 \\
80 & 0.022 & 0.792 & & 0.593 & 0.787 \\
75 & 0.012 & 0.851 & & 0.561 & 0.792 \\
\hline
\end{tabular}




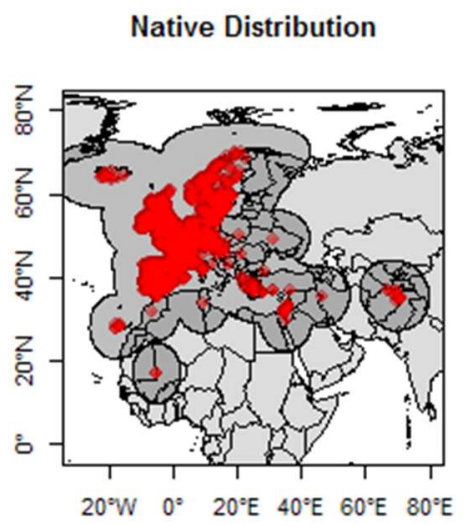

Introduced Distribution

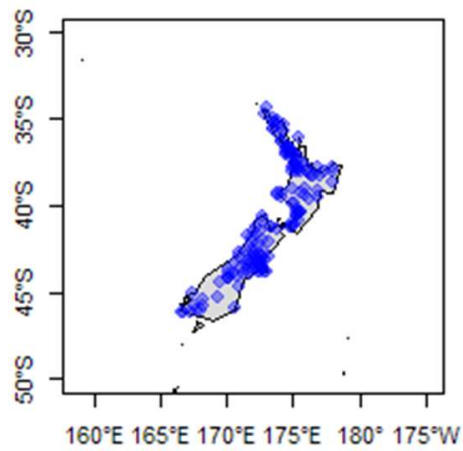

Native Climate Space

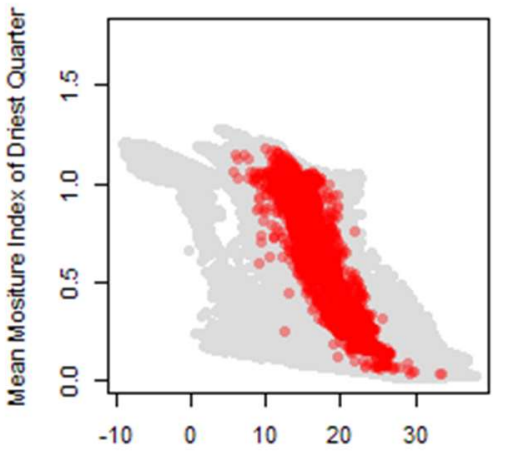

Mean Temperature of Warmest Quarter

Introduced Climate Space

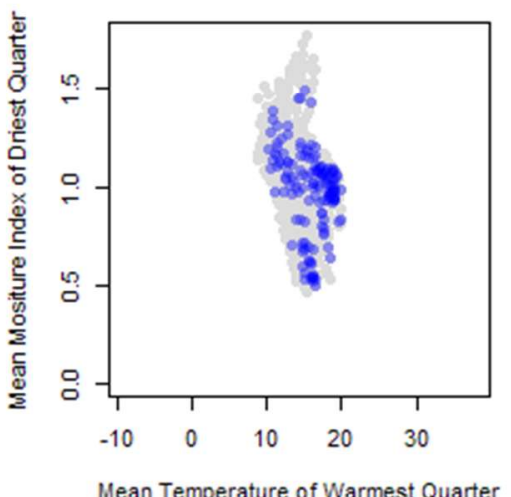

Native Climate Densities

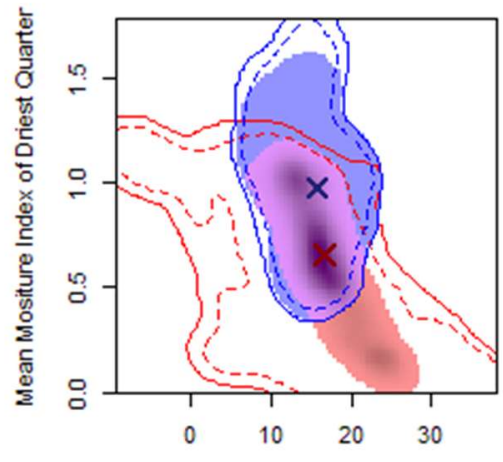

Mean Temperature of Warmest Quarter

Introduced Climate Densities

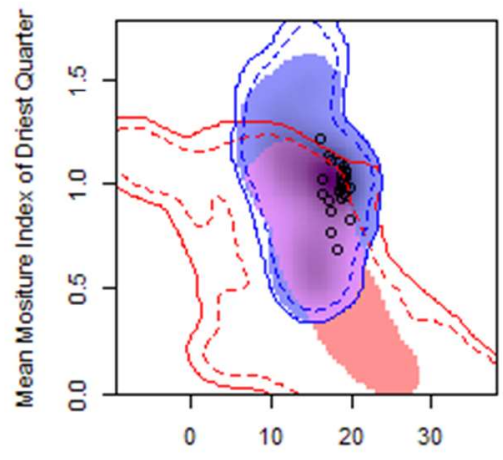

Mean Temperature of Warmest Quarter

Figure S28. Introduction of Cerastium glomeratum to New Zealand

Table S32. Niche shift indices for the introduction of Cerastium glomeratum to New Zealand

\begin{tabular}{cccccc}
\hline \multirow{2}{*}{$\begin{array}{c}\text { Marginal } \\
\text { climate }\end{array}$} & \multicolumn{2}{c}{ Niche Expansion } & & \multicolumn{2}{c}{ Niche Unfilling } \\
\cline { 2 - 3 } \cline { 5 - 6 } threshold $(\%)$ & $\begin{array}{c}\text { Analog } \\
\text { climate }\end{array}$ & $\begin{array}{c}\text { Non-analog } \\
\text { climate }\end{array}$ & & $\begin{array}{c}\text { Analog } \\
\text { climate }\end{array}$ & $\begin{array}{c}\text { Non-analog } \\
\text { climate }\end{array}$ \\
\hline 99 & 0.216 & 0.310 & & 0.001 & 0.168 \\
95 & 0.193 & 0.309 & & 0 & 0.172 \\
90 & 0.170 & 0.308 & & 0 & 0.178 \\
85 & 0.137 & 0.306 & & 0 & 0.185 \\
80 & 0.086 & 0.304 & & 0 & 0.195 \\
75 & 0.040 & 0.306 & & 0 & 0.206 \\
\hline
\end{tabular}




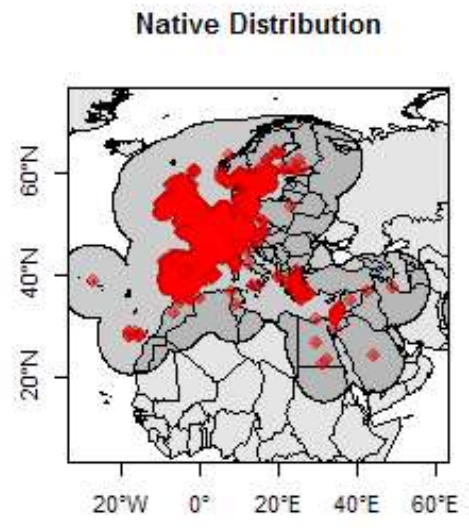

Introduced Distribution

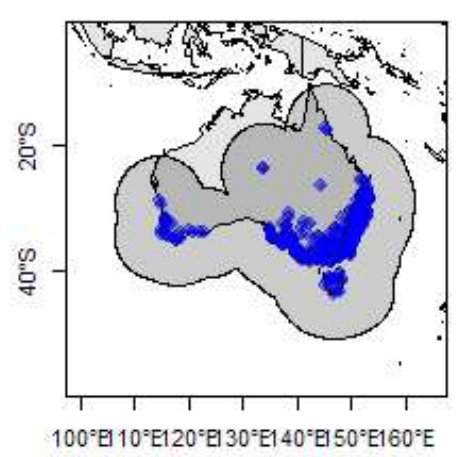

Native Climate Space

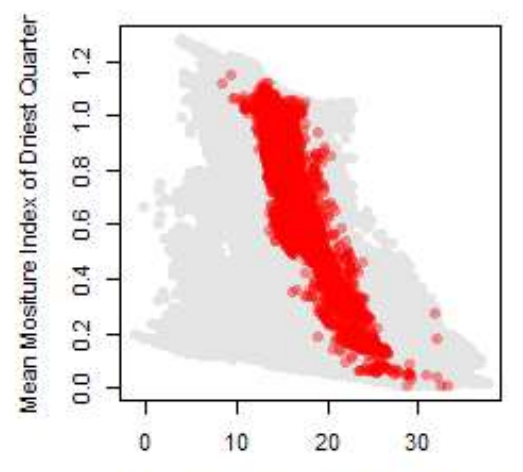

Mean Temperature of Warmest Quarter

Introduced Climate Space

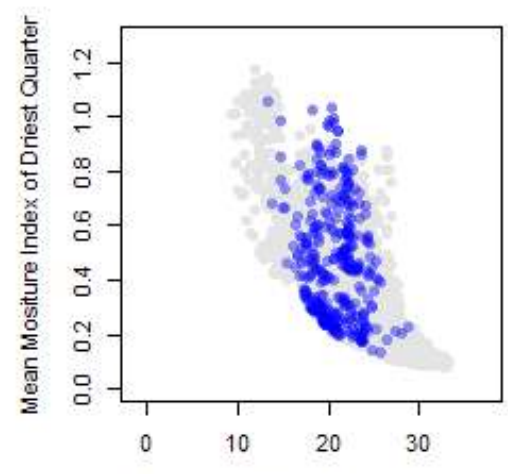

Mean Temperature of Warmest Quarter
Native Climate Densities

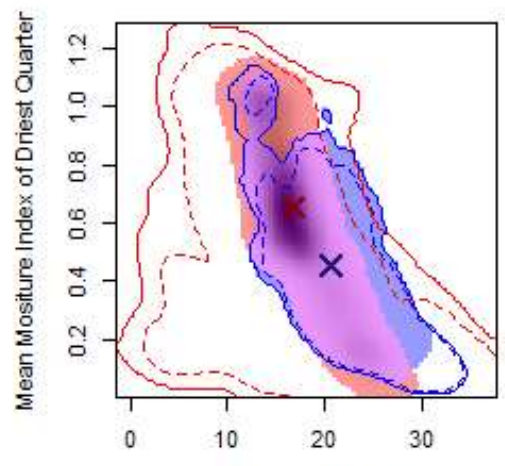

Mean Temperature of Warmest Quarter

Introduced Climate Densities

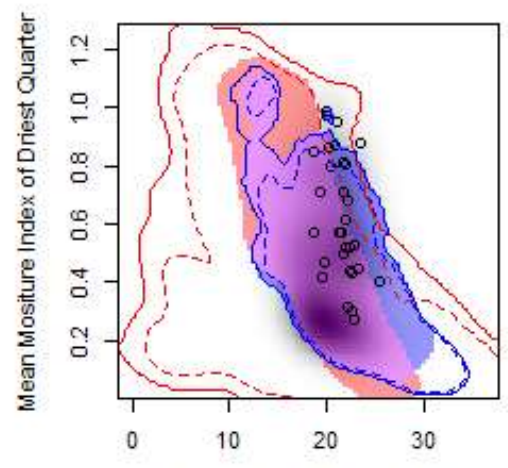

Mean Temperature of Warmest Quarter

Figure S29. Introduction of Euphorbia peplus to Australia

Table S33. Niche shift indices for the introduction of Euphorbia peplus to Australia

\begin{tabular}{cccccc}
\hline \multirow{2}{*}{$\begin{array}{c}\text { Marginal } \\
\text { climate } \\
\text { threshold }(\%)\end{array}$} & \multicolumn{2}{c}{\begin{tabular}{c} 
Niche Expansion \\
\cline { 2 - 3 } climate
\end{tabular}} & $\begin{array}{c}\text { Non-analog } \\
\text { climate }\end{array}$ & & \multicolumn{2}{c}{$\begin{array}{c}\text { Analog } \\
\text { climate }\end{array}$} & $\begin{array}{c}\text { Non-analog } \\
\text { climate }\end{array}$ \\
\hline 99 & 0.166 & 0.173 & & 0.019 & 0.119 \\
95 & 0.153 & 0.165 & & 0.016 & 0.146 \\
90 & 0.138 & 0.157 & & 0.011 & 0.175 \\
85 & 0.120 & 0.149 & & 0.005 & 0.210 \\
80 & 0.100 & 0.140 & & 0.001 & 0.251 \\
75 & 0.077 & 0.131 & & 0.001 & 0.302 \\
\hline
\end{tabular}




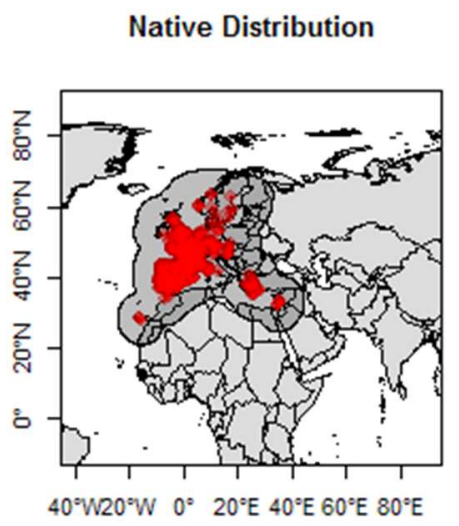

Introduced Distribution

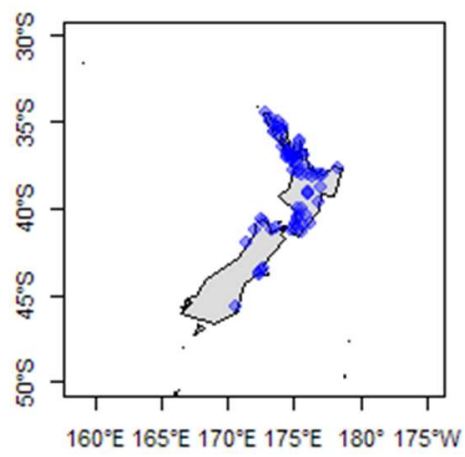

Native Climate Space

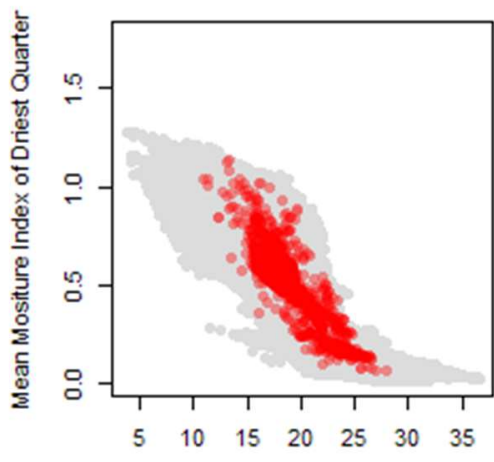

Mean Temperature of Warmest Quarter

Introduced Climate Space

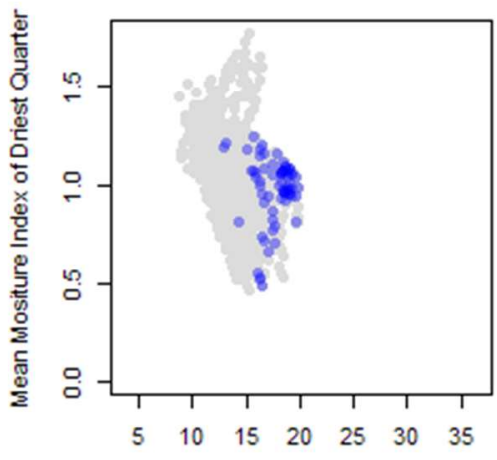

Mean Temperature of Warmest Quarter
Native Climate Densities

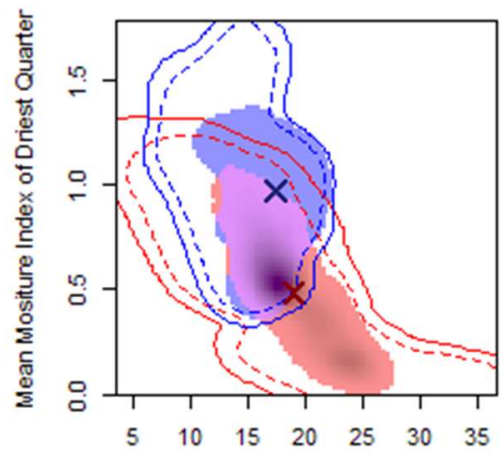

Mean Temperature of Warmest Quarter

Introduced Climate Densities

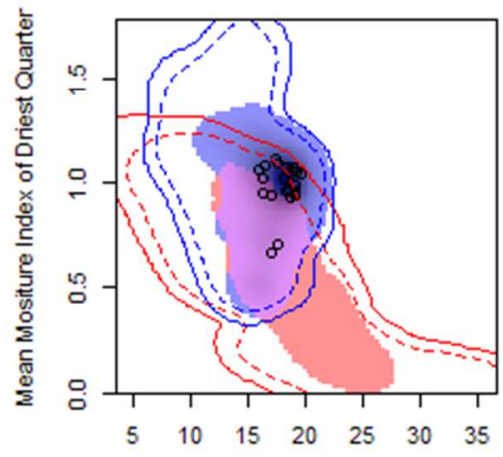

Mean Temperature of Warmest Quarter

Figure S30. Introduction of Lythrum hyssopifolia to New Zealand

Table S34. Niche shift indices for the introduction of Lythrum hyssopifolia to New Zealand

\begin{tabular}{cccccc}
\hline \multirow{2}{*}{$\begin{array}{c}\text { Marginal } \\
\text { climate }\end{array}$} & \multicolumn{2}{c}{ Niche Expansion } & & \multicolumn{2}{c}{ Niche Unfilling } \\
\cline { 2 - 3 } \cline { 5 - 6 } threshold $(\%)$ & $\begin{array}{c}\text { Analog } \\
\text { climate }\end{array}$ & $\begin{array}{c}\text { Non-analog } \\
\text { climate }\end{array}$ & & $\begin{array}{c}\text { Analog } \\
\text { climate }\end{array}$ & $\begin{array}{c}\text { Non-analog } \\
\text { climate }\end{array}$ \\
\hline 99 & 0.590 & 0.632 & & 0.130 & 0.403 \\
95 & 0.572 & 0.632 & & 0.111 & 0.403 \\
90 & 0.552 & 0.632 & & 0.091 & 0.403 \\
85 & 0.520 & 0.631 & & 0.071 & 0.408 \\
80 & 0.481 & 0.631 & & 0.044 & 0.418 \\
75 & 0.417 & 0.631 & & 0.021 & 0.430 \\
\hline
\end{tabular}




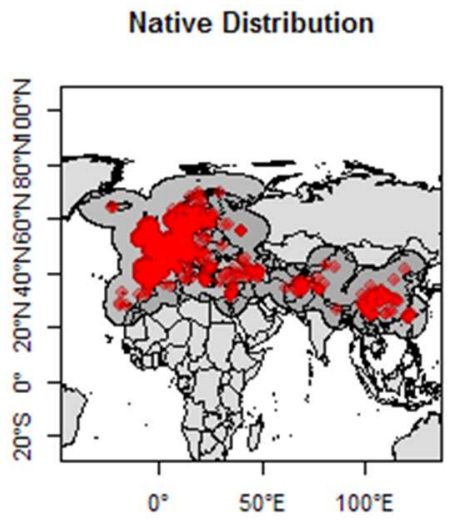

Introduced Distribution
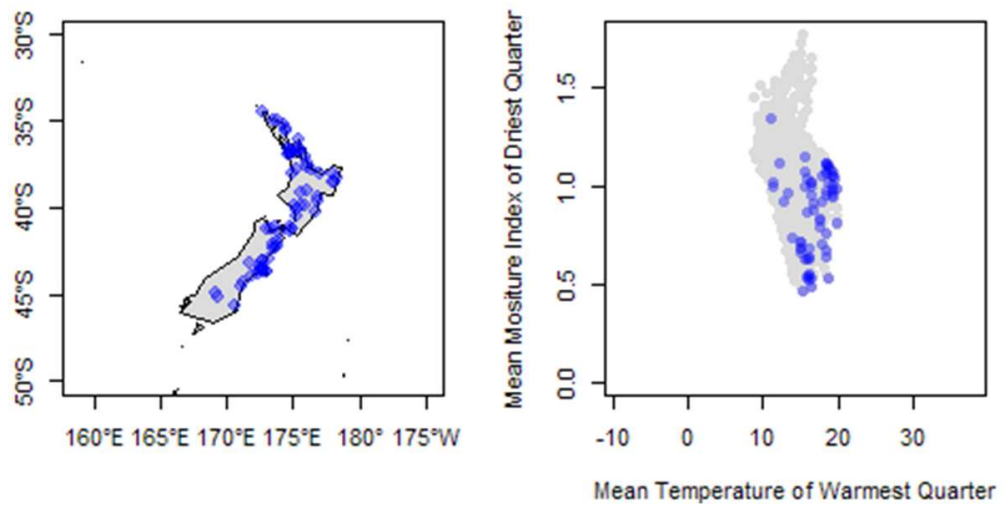

Native Climate Densities

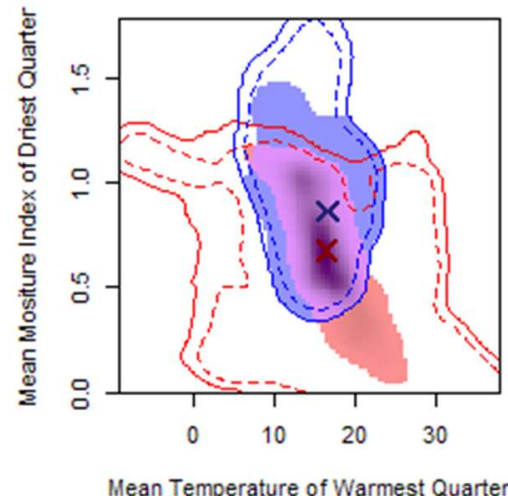

Introduced Climate Densities

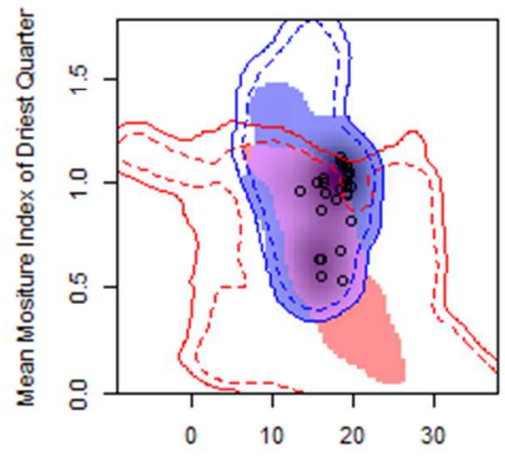

Mean Temperature of Warmest Quarter

Figure S31. Introduction of Medicago Iupulina to New Zealand

Table S35. Niche shift indices for the introduction of Medicago lupulina to New Zealand

\begin{tabular}{cccccc}
\hline \multirow{2}{*}{$\begin{array}{c}\text { Marginal } \\
\text { climate }\end{array}$} & \multicolumn{2}{c}{ Niche Expansion } & & \multicolumn{2}{c}{ Niche Unfilling } \\
\cline { 2 - 3 } \cline { 5 - 6 } threshold $(\%)$ & $\begin{array}{c}\text { Analog } \\
\text { climate }\end{array}$ & $\begin{array}{c}\text { Non-analog } \\
\text { climate }\end{array}$ & & $\begin{array}{c}\text { Analog } \\
\text { climate }\end{array}$ & $\begin{array}{c}\text { Non-analog } \\
\text { climate }\end{array}$ \\
\hline 99 & 0.215 & 0.271 & & 0.001 & 0.095 \\
95 & 0.190 & 0.268 & & 0.001 & 0.100 \\
90 & 0.167 & 0.266 & & 0.001 & 0.106 \\
85 & 0.134 & 0.263 & & 0.001 & 0.113 \\
80 & 0.084 & 0.268 & & 0.001 & 0.122 \\
75 & 0.049 & 0.298 & & 0.001 & 0.132 \\
\hline
\end{tabular}




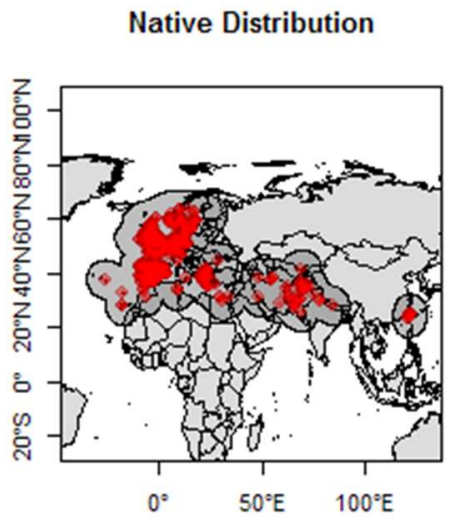

Introduced Distribution

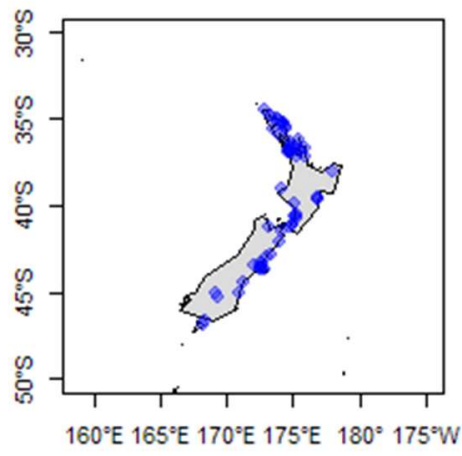

Figure S32. Introduction of Melilotus indicus to New Zealand
Native Climate Space

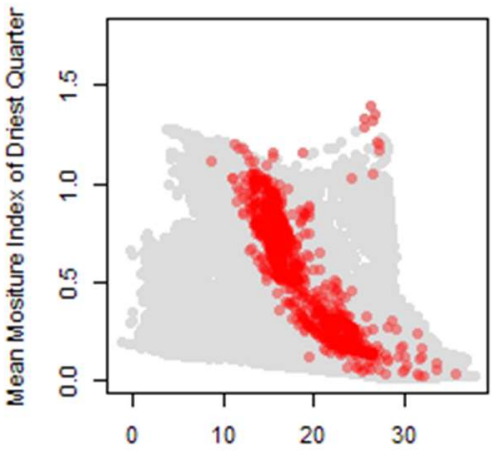

Mean Temperature of Warmest Quarter

Introduced Climate Space

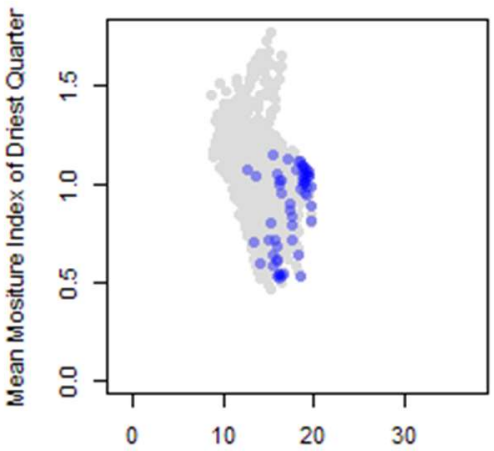

Mean Temperature of Warmest Quarter
Native Climate Densities

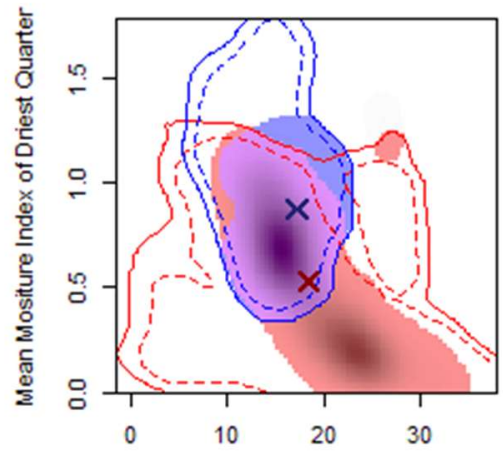

Mean Temperature of Warmest Quarter

Introduced Climate Densities

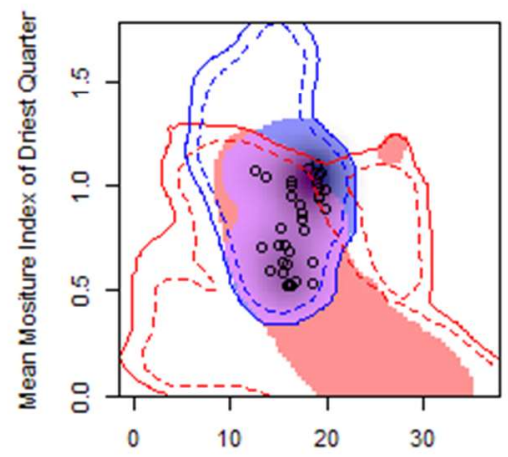

Mean Temperature of Warmest Quarter

Table S36. Niche shift indices for the introduction of Melilotus indicus to New Zealand

\begin{tabular}{cccccc}
\hline \multirow{2}{*}{$\begin{array}{c}\text { Marginal } \\
\text { climate }\end{array}$} & \multicolumn{2}{c}{ Niche Expansion } & & \multicolumn{2}{c}{ Niche Unfilling } \\
\cline { 2 - 3 } \cline { 5 - 6 } threshold $(\%)$ & $\begin{array}{c}\text { Analog } \\
\text { climate }\end{array}$ & $\begin{array}{c}\text { Non-analog } \\
\text { climate }\end{array}$ & & $\begin{array}{c}\text { Analog } \\
\text { climate }\end{array}$ & $\begin{array}{c}\text { Non-analog } \\
\text { climate }\end{array}$ \\
\hline 99 & 0.118 & 0.173 & & 0.007 & 0.385 \\
95 & 0.097 & 0.179 & & 0.007 & 0.391 \\
90 & 0.060 & 0.190 & & 0.007 & 0.397 \\
85 & 0.018 & 0.226 & & 0.007 & 0.406 \\
80 & 0.003 & 0.277 & & 0.007 & 0.414 \\
75 & 0 & 0.309 & & 0.006 & 0.423 \\
\hline
\end{tabular}



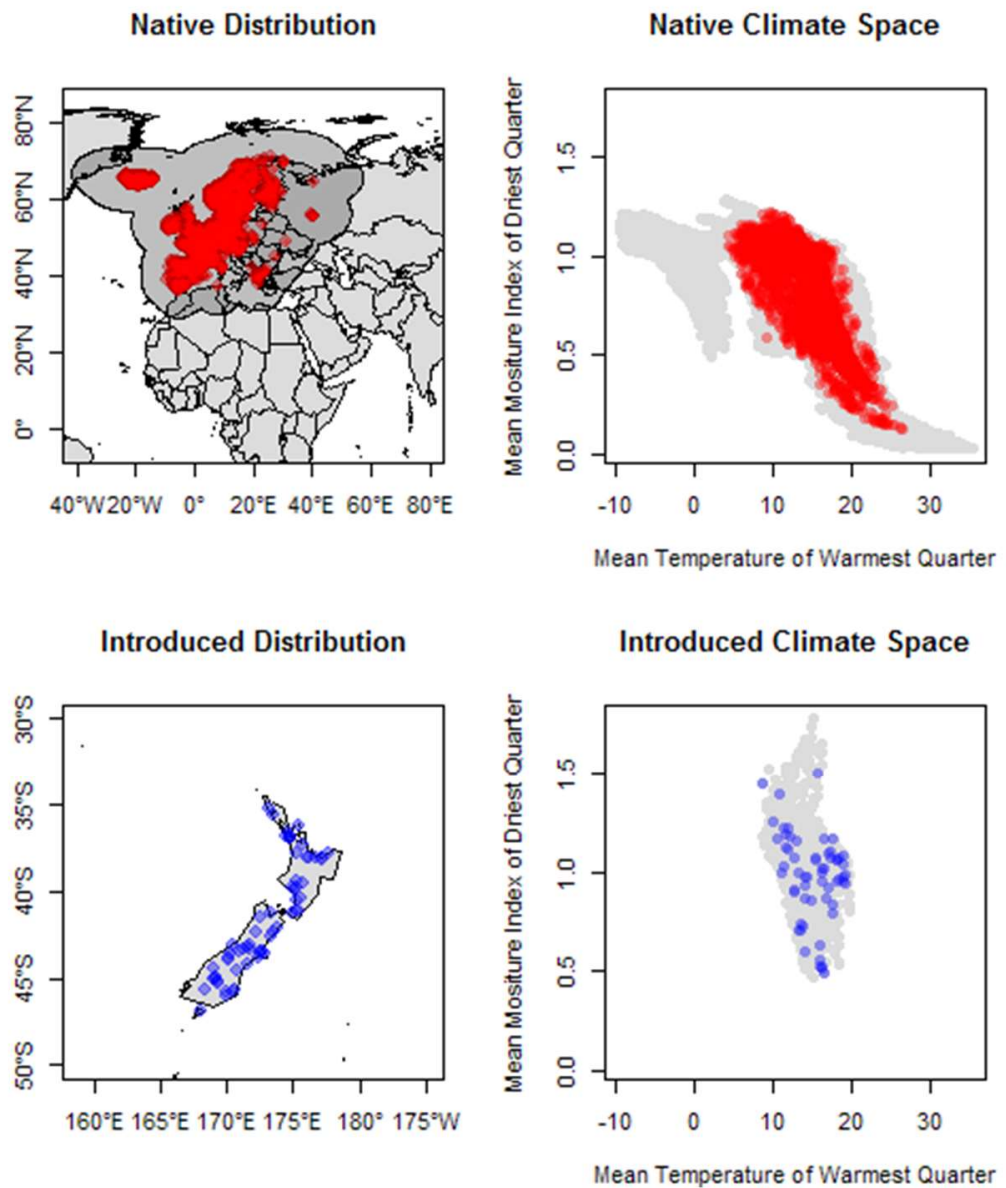

Native Climate Densities

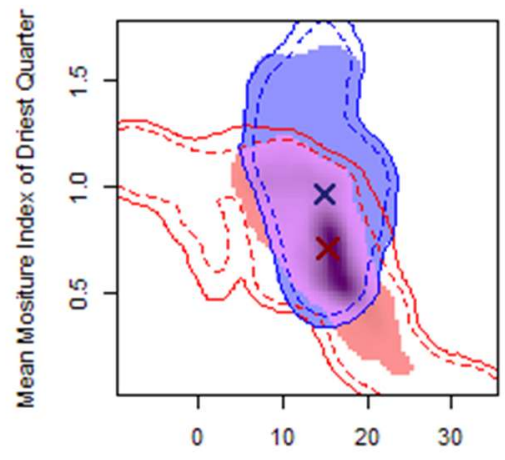

Mean Temperature of Warmest Quarter

Introduced Climate Densities

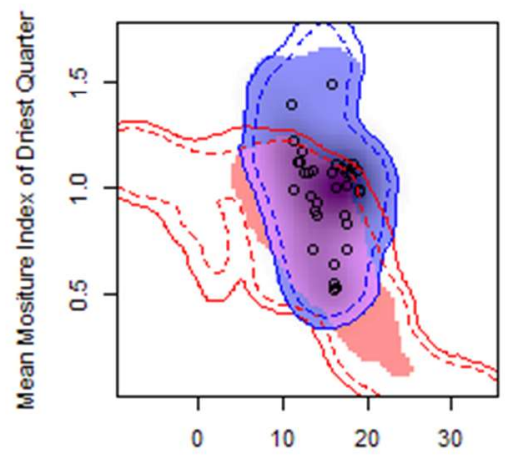

Mean Temperature of Warmest Quarter

Figure S33. Introduction of Myosotis arvensis to New Zealand

Table S37. Niche shift indices for the introduction of Myosotis arvensis to New Zealand

\begin{tabular}{cccccc}
\hline \multirow{2}{*}{$\begin{array}{c}\text { Marginal } \\
\text { climate }\end{array}$} & \multicolumn{2}{c}{ Niche Expansion } & & \multicolumn{2}{c}{ Niche Unfilling } \\
\cline { 2 - 3 } \cline { 5 - 6 } threshold $(\%)$ & $\begin{array}{c}\text { Analog } \\
\text { climate }\end{array}$ & $\begin{array}{c}\text { Non-analog } \\
\text { climate }\end{array}$ & & $\begin{array}{c}\text { Analog } \\
\text { climate }\end{array}$ & $\begin{array}{c}\text { Non-analog } \\
\text { climate }\end{array}$ \\
\hline 99 & 0.188 & 0.324 & & 0 & 0.048 \\
95 & 0.161 & 0.322 & & 0 & 0.054 \\
90 & 0.135 & 0.320 & & 0 & 0.063 \\
85 & 0.105 & 0.316 & & 0 & 0.073 \\
80 & 0.075 & 0.314 & & 0 & 0.084 \\
75 & 0.043 & 0.314 & & 0 & 0.098 \\
\hline
\end{tabular}




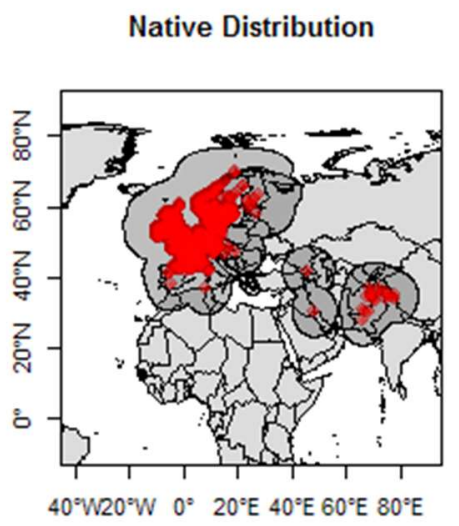

Introduced Distribution

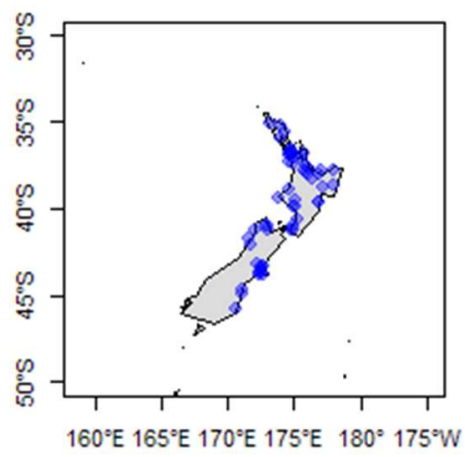

Native Climate Space

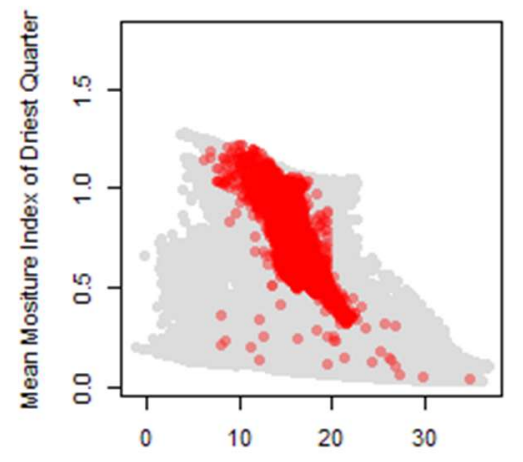

Mean Temperature of Warmest Quarter

Introduced Climate Space

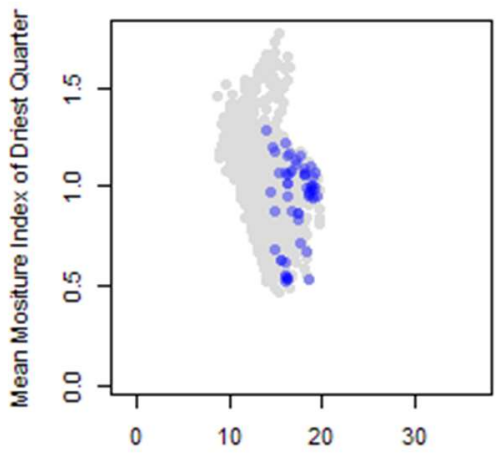

Mean Temperature of Warmest Quarter
Native Climate Densities

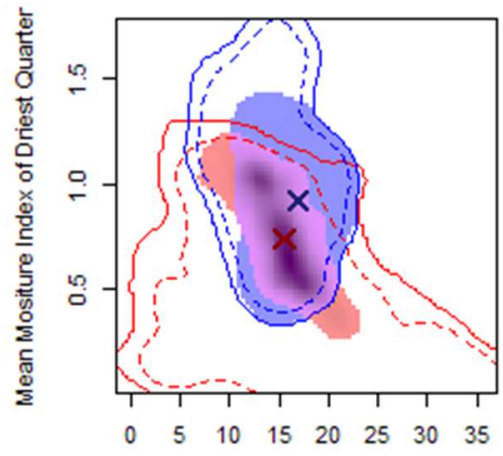

Mean Temperature of Warmest Quarter

Introduced Climate Densities

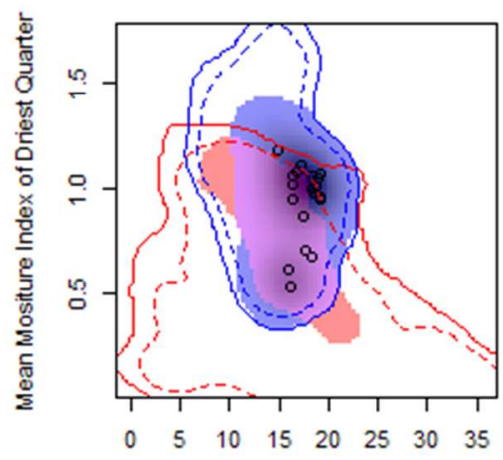

Mean Temperature of Warmest Quarter

Figure S34. Introduction of Persicaria maculosa to New Zealand

Table S38. Niche shift indices for the introduction of Persicaria maculosa to New Zealand

\begin{tabular}{cccccc}
\hline \multirow{2}{*}{$\begin{array}{c}\text { Marginal } \\
\text { climate }\end{array}$} & \multicolumn{2}{c}{ Niche Expansion } & & \multicolumn{2}{c}{ Niche Unfilling } \\
\cline { 2 - 3 } \cline { 5 - 6 } threshold $(\%)$ & $\begin{array}{c}\text { Analog } \\
\text { climate }\end{array}$ & $\begin{array}{c}\text { Non-analog } \\
\text { climate }\end{array}$ & & $\begin{array}{c}\text { Analog } \\
\text { climate }\end{array}$ & $\begin{array}{c}\text { Non-analog } \\
\text { climate }\end{array}$ \\
\hline 99 & 0.360 & 0.426 & & 0.017 & 0.038 \\
95 & 0.338 & 0.424 & & 0.017 & 0.042 \\
90 & 0.309 & 0.423 & & 0.017 & 0.047 \\
85 & 0.261 & 0.421 & & 0.017 & 0.052 \\
80 & 0.194 & 0.419 & & 0.017 & 0.060 \\
75 & 0.129 & 0.416 & & 0.017 & 0.069 \\
\hline
\end{tabular}




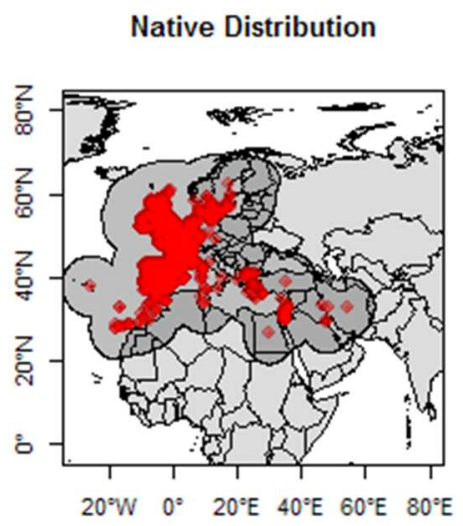

Introduced Distribution

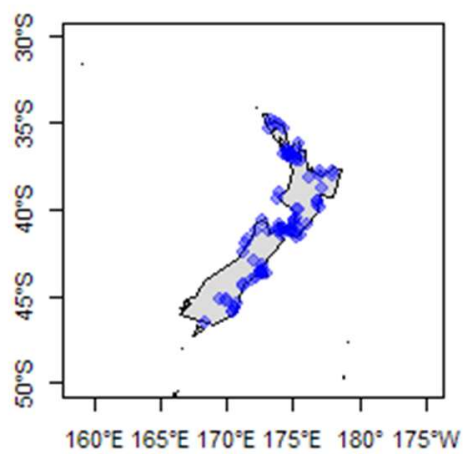

Native Climate Space

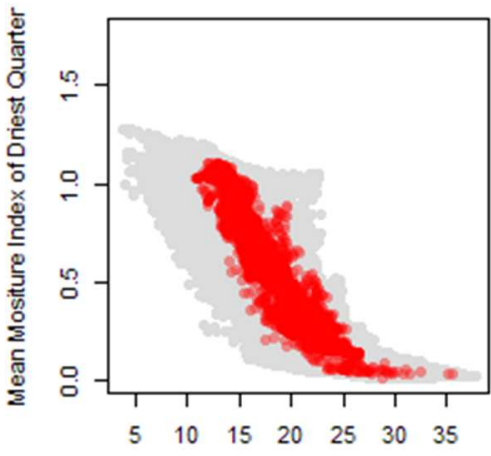

Mean Temperature of Warmest Quarter

Introduced Climate Space

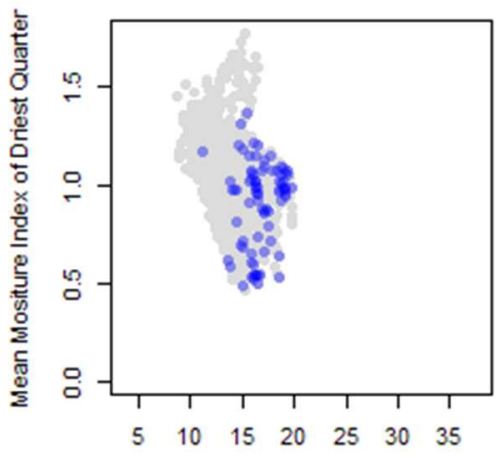

Mean Temperature of Warmest Quarter
Native Climate Densities

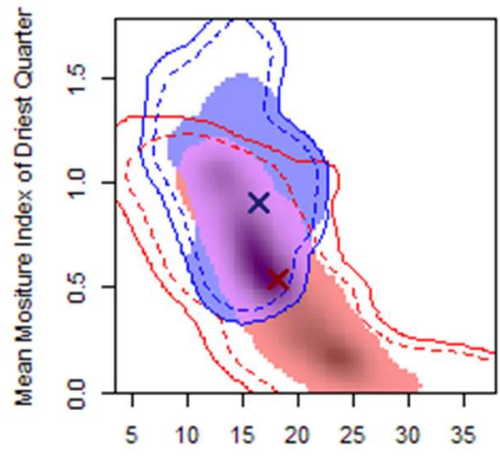

Mean Temperature of Warmest Quarter

Introduced Climate Densities

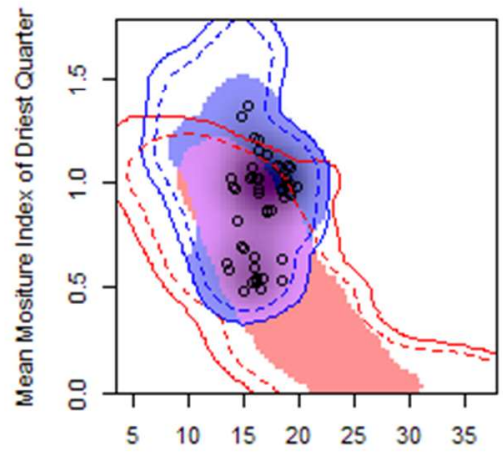

Mean Temperature of Warmest Quarter

Figure S35. Introduction of Plantago coronopus to New Zealand

Table S39. Niche shift indices for the introduction of Plantago coronopus to New Zealand

\begin{tabular}{cccccc}
\hline \multirow{2}{*}{$\begin{array}{c}\text { Marginal } \\
\text { climate }\end{array}$} & \multicolumn{2}{c}{ Niche Expansion } & & \multicolumn{2}{c}{ Niche Unfilling } \\
\cline { 2 - 3 } \cline { 5 - 6 } threshold $(\%)$ & $\begin{array}{c}\text { Analog } \\
\text { climate }\end{array}$ & $\begin{array}{c}\text { Non-analog } \\
\text { climate }\end{array}$ & & $\begin{array}{c}\text { Analog } \\
\text { climate }\end{array}$ & $\begin{array}{c}\text { Non-analog } \\
\text { climate }\end{array}$ \\
\hline 99 & 0.266 & 0.325 & & 0.001 & 0.332 \\
95 & 0.251 & 0.324 & & 0.001 & 0.341 \\
90 & 0.231 & 0.324 & & 0.002 & 0.352 \\
85 & 0.202 & 0.364 & & 0.002 & 0.364 \\
80 & 0.159 & 0.322 & & 0.002 & 0.378 \\
75 & 0.112 & 0.324 & & 0.002 & 0.393 \\
\hline
\end{tabular}




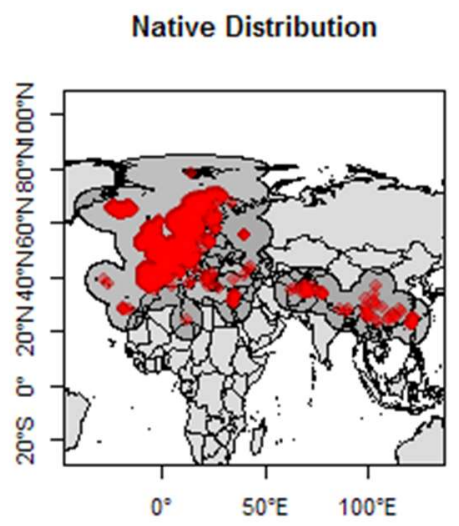

Introduced Distribution

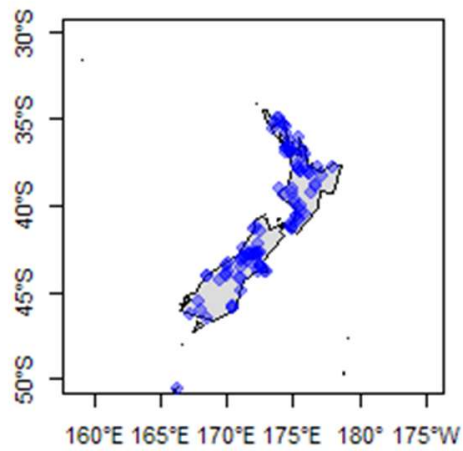

Figure S36. Introduction of Poa annua to New Zealand

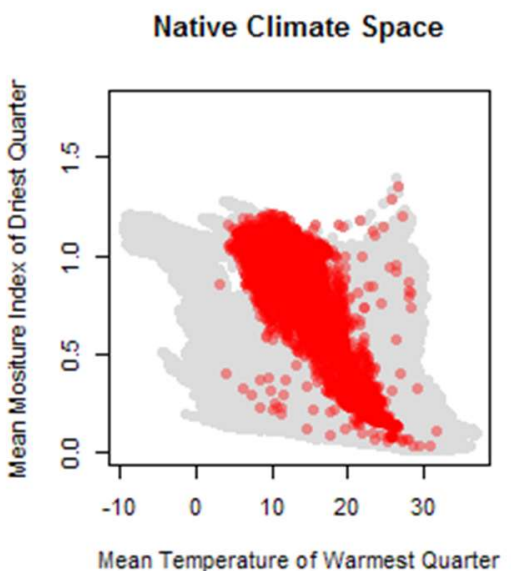

Introduced Climate Space

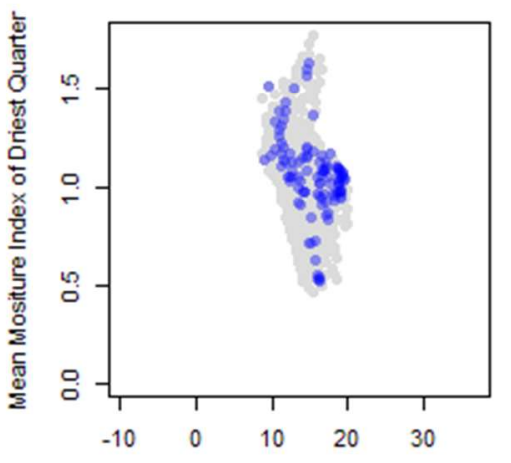

Mean Temperature of Warmest Quarter

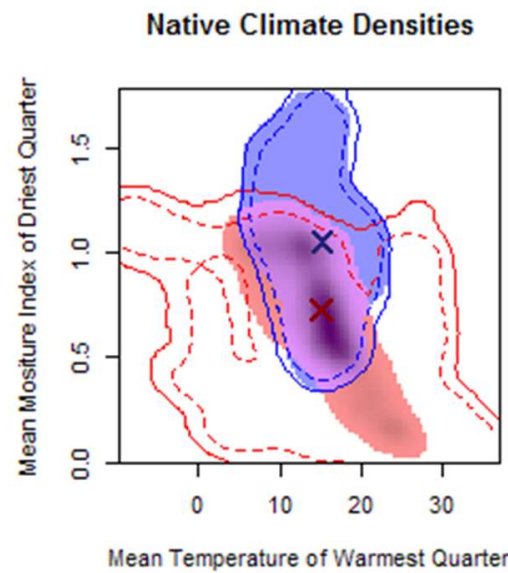

Introduced Climate Densities

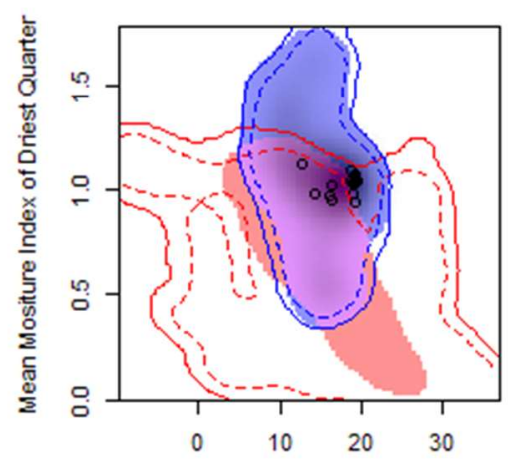

Mean Temperature of Warmest Quarter

Table S40. Niche shift indices for the introduction of Poa annua to New Zealand

\begin{tabular}{cccccc}
\hline \multirow{2}{*}{$\begin{array}{c}\text { Marginal } \\
\text { climate }\end{array}$} & \multicolumn{2}{c}{ Niche Expansion } & & \multicolumn{2}{c}{ Niche Unfilling } \\
\cline { 2 - 3 } \cline { 5 - 6 } threshold $(\%)$ & $\begin{array}{c}\text { Analog } \\
\text { climate }\end{array}$ & $\begin{array}{c}\text { Non-analog } \\
\text { climate }\end{array}$ & & $\begin{array}{c}\text { Analog } \\
\text { climate }\end{array}$ & $\begin{array}{c}\text { Non-analog } \\
\text { climate }\end{array}$ \\
\hline 99 & 0.194 & 0.346 & & 0.023 & 0.139 \\
95 & 0.152 & 0.345 & & 0.018 & 0.143 \\
90 & 0.106 & 0.351 & & 0.013 & 0.150 \\
85 & 0.069 & 0.387 & & 0.009 & 0.160 \\
80 & 0.022 & 0.422 & & 0.007 & 0.170 \\
75 & 0.006 & 0.465 & & 0.003 & 0.184 \\
\hline
\end{tabular}



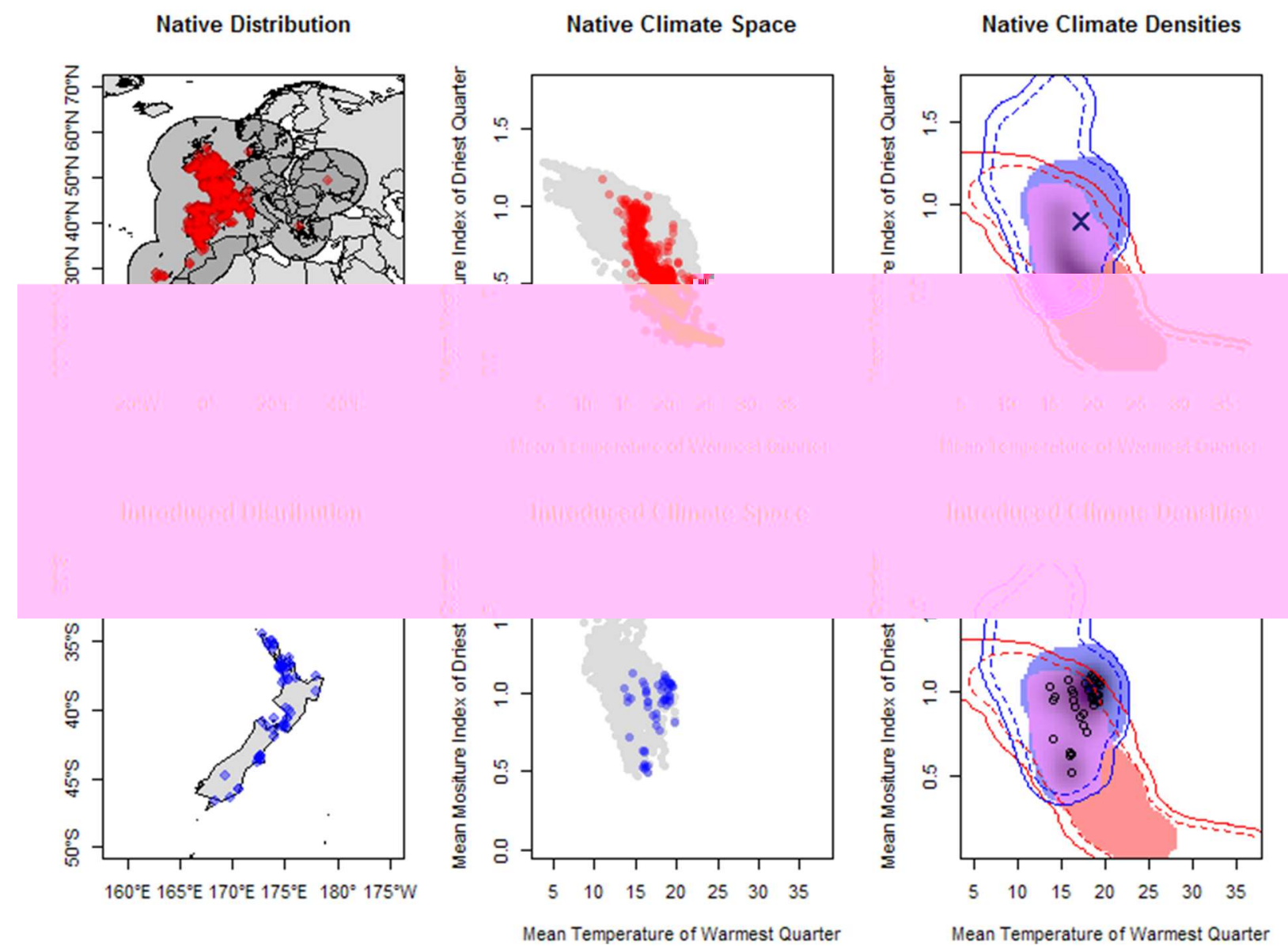

Figure S37. Introduction of Ranunculus parviflorus to New Zealand

Table S41. Niche shift indices for the introduction of Ranunculus parviflorus to New Zealand

\begin{tabular}{cccccc}
\hline \multirow{2}{*}{$\begin{array}{c}\text { Marginal } \\
\text { climate } \\
\text { threshold }(\%)\end{array}$} & \multicolumn{2}{c}{ Niche Expansion } & & \multicolumn{2}{c}{ Niche Unfilling } \\
\cline { 2 - 3 } \cline { 5 - 6 } & climate & $\begin{array}{c}\text { Non-analog } \\
\text { climate }\end{array}$ & & $\begin{array}{c}\text { Analog } \\
\text { climate }\end{array}$ & $\begin{array}{c}\text { Non-analog } \\
\text { climate }\end{array}$ \\
\hline 99 & 0.295 & 0.355 & & 0.045 & 0.318 \\
95 & 0.275 & 0.354 & & 0.033 & 0.323 \\
90 & 0.253 & 0.354 & & 0.021 & 0.333 \\
85 & 0.224 & 0.352 & & 0.010 & 0.345 \\
80 & 0.186 & 0.351 & & 0 & 0.368 \\
75 & 0.139 & 0.349 & & 0 & 0.393 \\
\hline
\end{tabular}




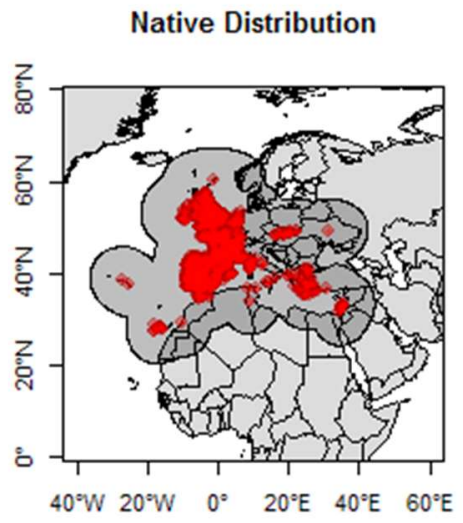

Introduced Distribution

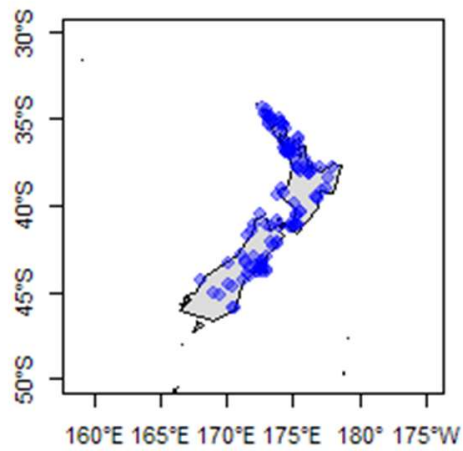

Native Climate Space

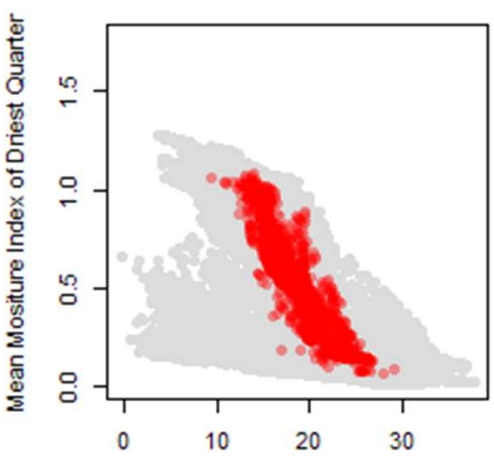

Mean Temperature of Warmest Quarter

Introduced Climate Space

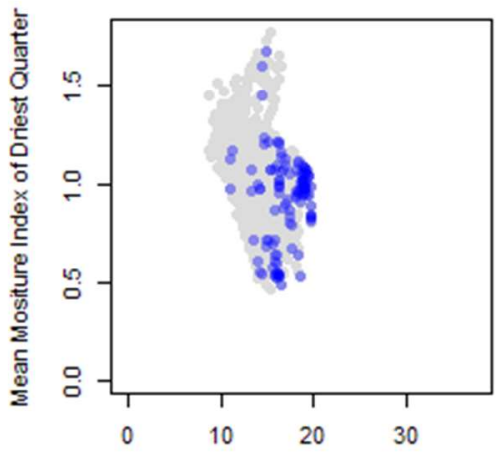

Mean Temperature of Warmest Quarter
Native Climate Densities

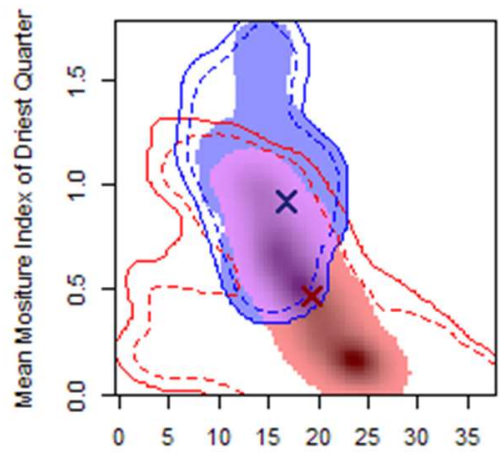

Mean Temperature of Warmest Quarte

Introduced Climate Densities

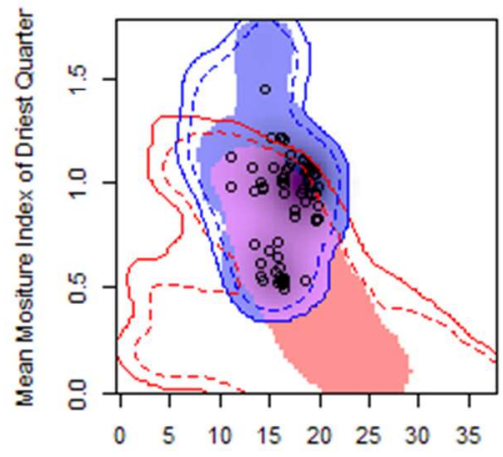

Mean Temperature of Warmest Quarter

Figure S38. Introduction of Silene gallica to New Zealand

Table S42. Niche shift indices for the introduction of Silene gallica to New Zealand

\begin{tabular}{cccccc}
\hline \multirow{2}{*}{$\begin{array}{c}\text { Marginal } \\
\text { climate } \\
\text { threshold (\%) }\end{array}$} & \multicolumn{2}{c}{ Niche Expansion } & & \multicolumn{2}{c}{ Niche Unfilling } \\
\cline { 2 - 3 } \cline { 5 - 6 } & $\begin{array}{c}\text { Analog } \\
\text { climate }\end{array}$ & $\begin{array}{c}\text { Non-analog } \\
\text { climate }\end{array}$ & & $\begin{array}{c}\text { Analog } \\
\text { climate }\end{array}$ & $\begin{array}{c}\text { Non-analog } \\
\text { climate }\end{array}$ \\
\hline 99 & 0.234 & 0.329 & & 0 & 0.458 \\
95 & 0.211 & 0.328 & & 0 & 0.473 \\
90 & 0.188 & 0.326 & & 0 & 0.489 \\
85 & 0.156 & 0.324 & & 0 & 0.503 \\
80 & 0.124 & 0.322 & & 0 & 0.521 \\
75 & 0.093 & 0.324 & & 0 & 0.539 \\
\hline
\end{tabular}




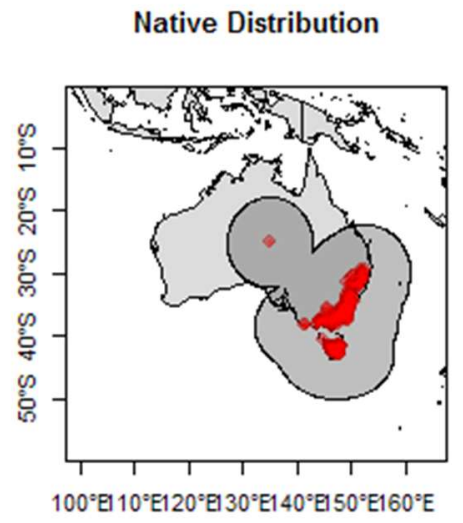

Introduced Distribution

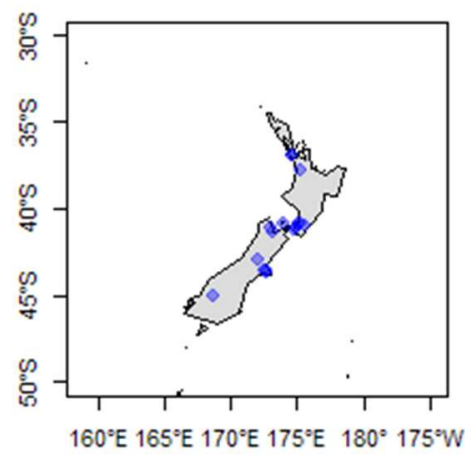

Native Climate Space

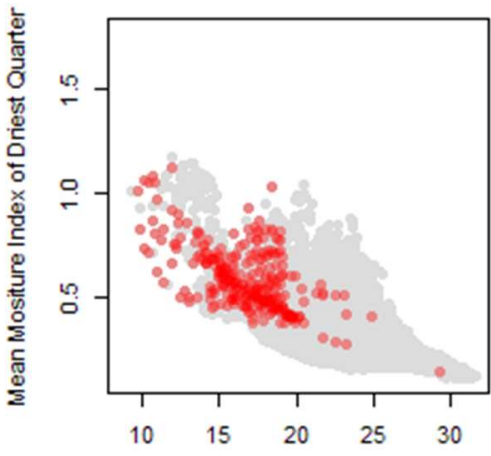

Mean Temperature of Warmest Quarter Introduced Climate Space

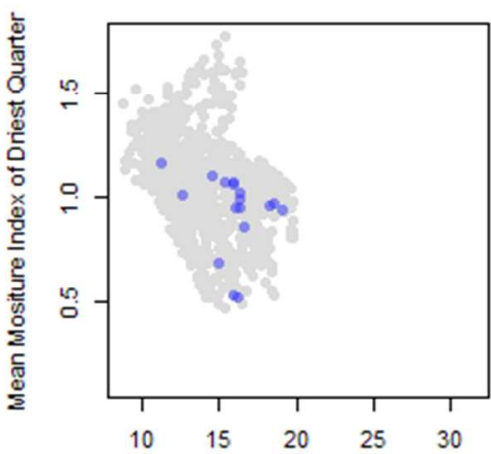

Mean Temperature of Warmest Quarter
Native Climate Densities

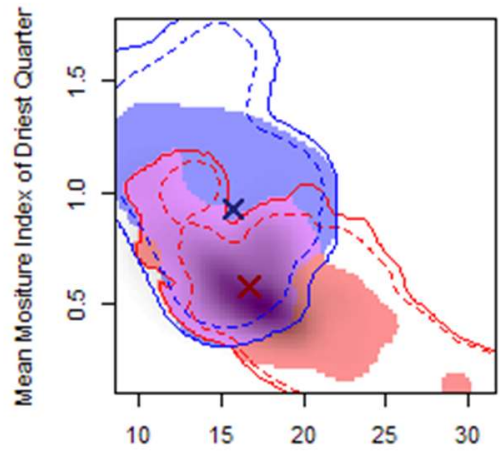

Mean Temperature of Warmest Quarter

Introduced Climate Densities

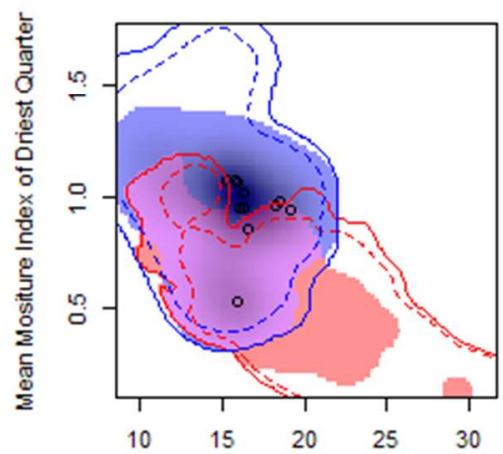

Mean Temperature of Warmest Quarter

Figure S39. Introduction of Solenogyne gunnii to New Zealand

Table S43. Niche shift indices for the introduction of Solenogyne gunnii to New Zealand

\begin{tabular}{cccccc}
\hline \multirow{2}{*}{$\begin{array}{c}\text { Marginal } \\
\text { climate }\end{array}$} & \multicolumn{2}{c}{ Niche Expansion } & & \multicolumn{2}{c}{ Niche Unfilling } \\
\cline { 2 - 3 } \cline { 5 - 6 } threshold $(\%)$ & $\begin{array}{c}\text { Analog } \\
\text { climate }\end{array}$ & $\begin{array}{c}\text { Non-analog } \\
\text { climate }\end{array}$ & & $\begin{array}{c}\text { Analog } \\
\text { climate }\end{array}$ & $\begin{array}{c}\text { Non-analog } \\
\text { climate }\end{array}$ \\
\hline 99 & 0.072 & 0.407 & & 0 & 0.122 \\
95 & 0.072 & 0.453 & & 0 & 0.150 \\
90 & 0.069 & 0.494 & & 0 & 0.183 \\
85 & 0.063 & 0.547 & & 0 & 0.224 \\
80 & 0.064 & 0.597 & & 0 & 0.268 \\
75 & 0.059 & 0.650 & & 0 & 0.315 \\
\hline
\end{tabular}




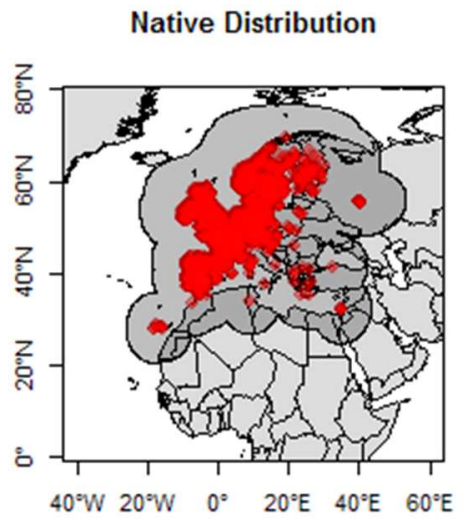

Introduced Distribution

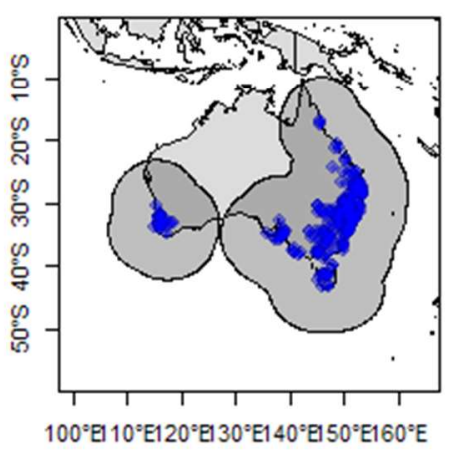

Figure S40. Introduction of Stachys arvensis to Australia
Native Climate Space

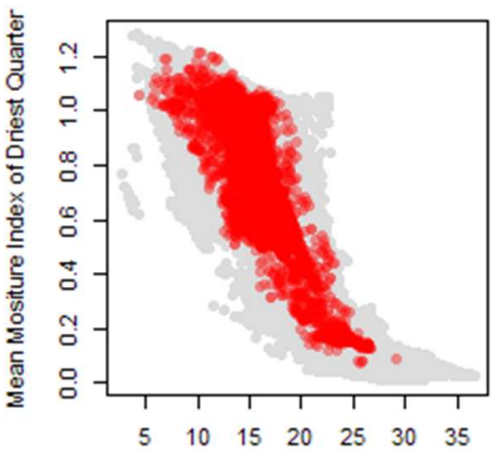

Mean Temperature of Warmest Quarter

Introduced Climate Space

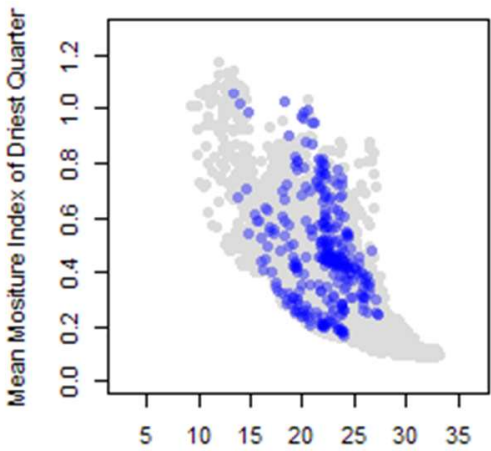

Mean Temperature of Warmest Quarter

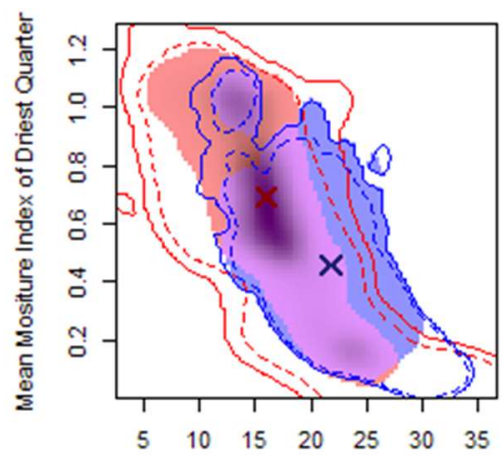

Mean Temperature of Warmest Quarter

Introduced Climate Densities

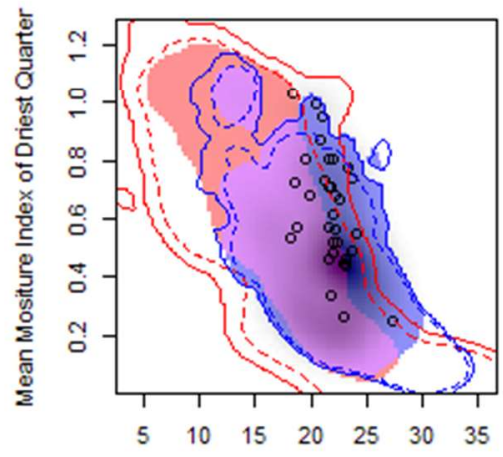

Mean Temperature of Warmest Quarter

Table S44. Niche shift indices for the introduction of Stachys arvensis to Australia

\begin{tabular}{cccccc}
\hline \multirow{2}{*}{$\begin{array}{c}\text { Marginal } \\
\text { climate }\end{array}$} & \multicolumn{2}{c}{ Niche Expansion } & & \multicolumn{2}{c}{ Niche Unfilling } \\
\cline { 2 - 3 } \cline { 5 - 6 } threshold $(\%)$ & $\begin{array}{c}\text { Analog } \\
\text { climate }\end{array}$ & $\begin{array}{c}\text { Non-analog } \\
\text { climate }\end{array}$ & & $\begin{array}{c}\text { Analog } \\
\text { climate }\end{array}$ & $\begin{array}{c}\text { Non-analog } \\
\text { climate }\end{array}$ \\
\hline 99 & 0.268 & 0.326 & & 0.009 & 0.095 \\
95 & 0.252 & 0.325 & & 0.008 & 0.098 \\
90 & 0.230 & 0.323 & & 0.007 & 0.101 \\
85 & 0.189 & 0.320 & & 0.005 & 0.106 \\
80 & 0.131 & 0.318 & & 0.004 & 0.114 \\
75 & 0.060 & 0.314 & & 0.003 & 0.123 \\
\hline
\end{tabular}




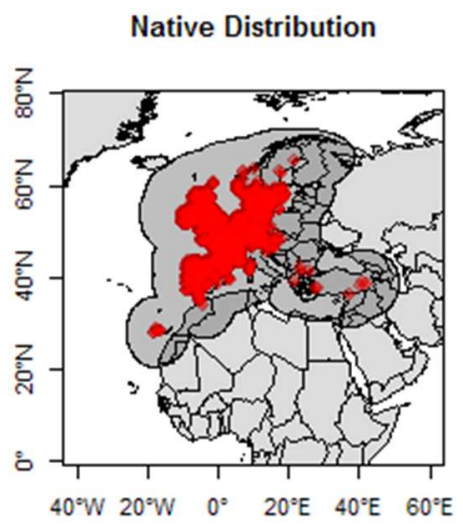

Introduced Distribution

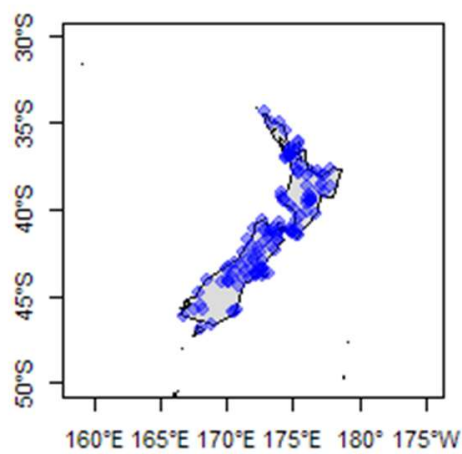

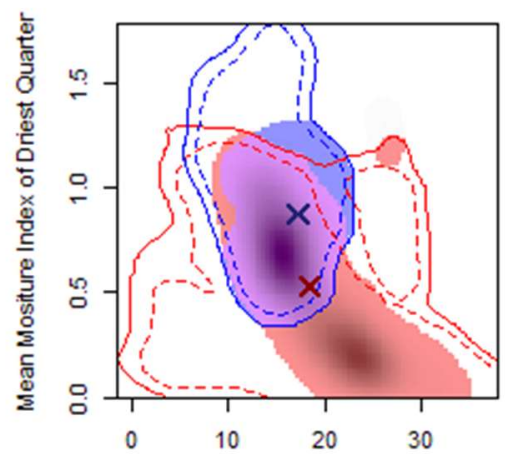

Mean Temperature of Warmest Quarter

Introduced Climate Densities

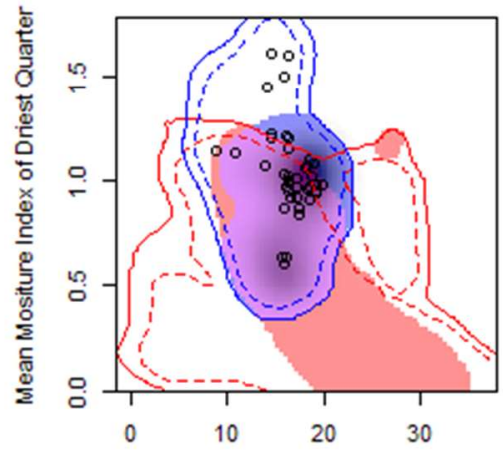

Mean Temperature of Warmest Quarter

Figure S41. Introduction of Trifolium dubium to New Zealand

Table S45. Niche shift indices for the introduction of Trifolium dubium to New Zealand

\begin{tabular}{cccccc}
\hline \multirow{2}{*}{$\begin{array}{c}\text { Marginal } \\
\text { climate }\end{array}$} & \multicolumn{2}{c}{ Niche Expansion } & & \multicolumn{2}{c}{ Niche Unfilling } \\
\cline { 2 - 3 } \cline { 5 - 6 } threshold (\%) & $\begin{array}{c}\text { Analog } \\
\text { climate }\end{array}$ & $\begin{array}{c}\text { Non-analog } \\
\text { climate }\end{array}$ & & $\begin{array}{c}\text { Analog } \\
\text { climate }\end{array}$ & $\begin{array}{c}\text { Non-analog } \\
\text { climate }\end{array}$ \\
\hline 99 & 0.304 & 0.387 & & 0.003 & 0.070 \\
95 & 0.288 & 0.386 & & 0.001 & 0.073 \\
90 & 0.269 & 0.385 & & 0 & 0.078 \\
85 & 0.246 & 0.383 & & 0 & 0.084 \\
80 & 0.207 & 0.380 & & 0 & 0.093 \\
75 & 0.158 & 0.376 & & 0 & 0.106 \\
\hline
\end{tabular}




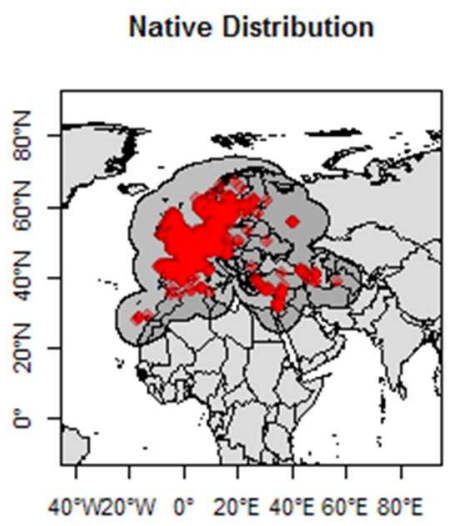

Introduced Distribution

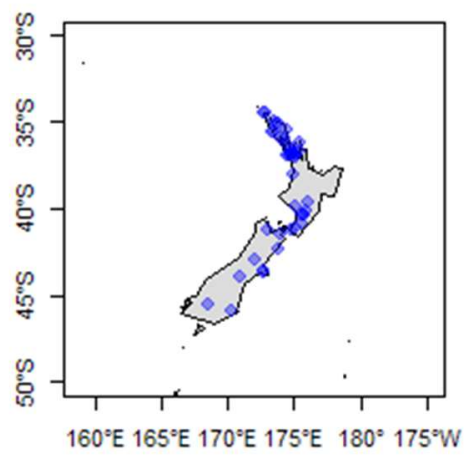

Native Climate Space

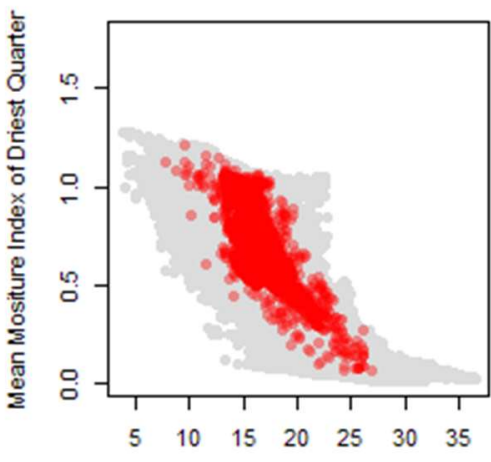

Mean Temperature of Warmest Quarter

Introduced Climate Space

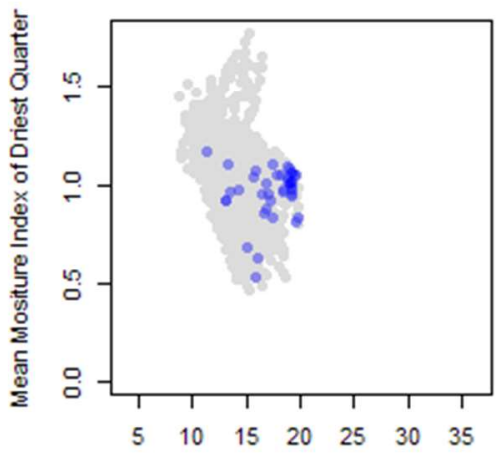

Mean Temperature of Warmest Quarter
Native Climate Densities

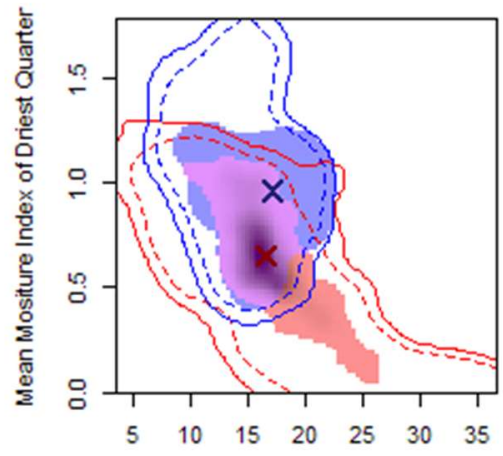

Mean Temperature of Warmest Quarter

Introduced Climate Densities

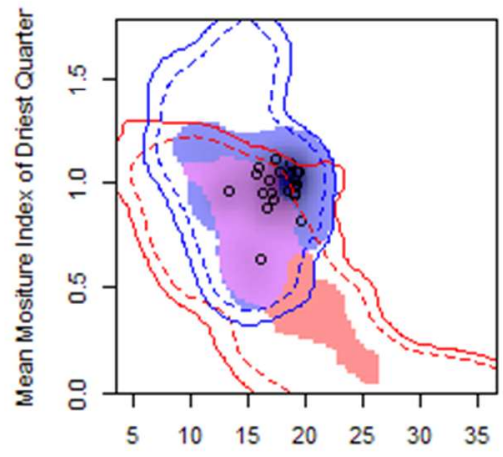

Mean Temperature of Warmest Quarter

Figure S42. Introduction of Vicia tetrasperma to New Zealand

Table S46. Niche shift indices for the introduction of Vicia tetrasperma to New Zealand

\begin{tabular}{cccccc}
\hline \multirow{2}{*}{$\begin{array}{c}\text { Marginal } \\
\text { climate }\end{array}$} & \multicolumn{2}{c}{ Niche Expansion } & & \multicolumn{2}{c}{ Niche Unfilling } \\
\cline { 2 - 3 } \cline { 5 - 6 } threshold $(\%)$ & $\begin{array}{c}\text { Analog } \\
\text { climate }\end{array}$ & $\begin{array}{c}\text { Non-analog } \\
\text { climate }\end{array}$ & & $\begin{array}{c}\text { Analog } \\
\text { climate }\end{array}$ & $\begin{array}{c}\text { Non-analog } \\
\text { climate }\end{array}$ \\
\hline 99 & 0.476 & 0.508 & & 0.090 & 0.143 \\
95 & 0.458 & 0.508 & & 0.084 & 0.143 \\
90 & 0.434 & 0.507 & & 0.079 & 0.143 \\
85 & 0.390 & 0.505 & & 0.072 & 0.143 \\
80 & 0.317 & 0.503 & & 0.061 & 0.143 \\
75 & 0.224 & 0.501 & & 0.047 & 0.143 \\
\hline
\end{tabular}




\title{
5. Rapid loss of phenotypic plasticity in the introduced range of the beach daisy, Arctotheca populifolia
}

\begin{abstract}
Phenotypic plasticity is thought to be beneficial for species when introduced to a new range, increasing their proficiency in colonising and expanding into new environments. It has been hypothesised that an increase in phenotypic plasticity can be selected for when introduced to a new range. I aim to test if there is greater phenotypic plasticity in the introduced populations of Arctotheca populifolia, compared to their native source population. I compared levels of phenotypic plasticity in four introduced Australian populations with the one identified source population from South Africa, across two separate glasshouse experiments that varied availability of, i) nutrients and ii) water. Phenotypic plasticity was assessed at the population level for thirteen individual traits, and across a suite of traits using a meta-analysis to determine whether there was an overall difference in plasticity between ranges. The Australian populations had significantly lower composite plasticity in response to nutrient availability (0.47 [95\% Cl: $-0.84 ;-0.09]$ ), as well as lower plasticity in response to water availability, though this was not significant $(-0.26$ [95\% Cl: $-0.56 ; 0.05])$. Contrary to my expectations, Arctotheca populifolia has experienced a loss of phenotypic plasticity in as little as 80 years since its introduction to Australia. This rapid loss of plasticity could have been rapidly selected for in the introduced range, or occurred due to founder effects on introduction.
\end{abstract}




\section{$\underline{5.1 \text { Introduction }}$}

Phenotypic plasticity is the capacity for a single genotype to exhibit multiple phenotypes in response to varying environmental conditions (Bradshaw 1965; Pigliucci 2001; Whitman \& Agrawal 2009). It is a key way in which plants can adjust to environmental change (Schlichting 1986), and enable them to express advantageous phenotypes over a greater range of environments (Bradshaw 1965; Donohue et al. 2001; Sultan 2001; Richards et al. 2006). Introduced species are often considered to show higher phenotypic plasticity than their native conspecifics, increasing their proficiency in colonising and expanding into new environments (Sexton et al. 2002; Richards et al. 2006; Nicotra et al. 2010; Davidson et al. 2011). This idea dates back to Baker's (1965) description of the 'ideal weed', in which a species can succeed in a wide range of environments through high phenotypic plasticity, enabling individuals to alter their phenotype to better match the new environment (Williams et al. 1995; Parker et al. 2002; Lamarque et al. 2013).

Introduced species may also adapt to new environmental conditions, through rapid evolution to novel selection pressures in the introduced range (Muller-Scharer et al. 2004; Lavergne \& Molofsky 2007; Buswell et al. 2011). It has been suggested that rapid evolution and phenotypic plasticity can act in concert, whereby pre-adapted phenotypic plasticity may enable a colonising species to initially persist in a new environment, and then subsequently adapt to it over a number of generations (Pigliucci 2001; Schlichting 2004; Lande 2009). These two modes to cope with a change in environment should not be viewed independently, as phenotypic plasticity can itself be considered a property of the genotype that can undergo selection and evolve (West-Eberhard 2003; Lande 2009; Crispo et al. 2010). The idea that phenotypic plasticity can evolve rapidly in non-native species has been predicted as a key way in which they can broaden their niche and succeed in the introduced range (Richards et al. 2006; Bossdorf et al. 2005; Zou et al. 2009).

It has been hypothesised that an increase in phenotypic plasticity may be rapidly selected for in introduced populations, via stress release (Huang et al. 2015) or through a transient 
increase in plasticity that accelerates evolution towards a new optimal phenotype (GomezMestre \& Jovani 2013; Lande 2015). Higher levels of phenotypic plasticity have been observed in introduced plant populations when compared to conspecifics from the native range (Kaufman \& Smouse 2001; Bossdorf et al. 2008; Hylgaard \& Brix 2012), supporting the idea that plasticity can be rapidly selected for in the introduced range. In other cases, no difference in plasticity was observed between introduced and native populations of Acer negundo (Lamarque et al. 2013) or Microstegium vimineim (Flory et al. 2011). Bossdorf et al. (2005) documented an increase in plasticity in introduced plants when compared to conspecifics from their native range in 5 out of 10 studies, with the remainder showing no change. Given these mixed results, the role that phenotypic plasticity may play in facilitating persistence and subsequent expansion of a non-native species in the introduced range remains unclear. The overall aim of this study is to test for a difference in plasticity in populations of an introduced plant, relative to its native source population.

Phenotypic plasticity of a given trait may express itself differently in response to variation across different environmental conditions (Bradshaw 1965; Pigliucci 2001). For example, specific leaf area in Rubia peregrina showed a plastic response to light and nutrient availability, but not to water (Navas \& Garner 2002). Consequently, testing for plasticity across multiple experimental treatments can give a better picture of the overall plasticity that a population may possess. Nutrient and water availability are key resources for plant growth and survival, and can both have a strong influence on a plant's phenotypic expression and growth strategy (Coleman et al. 1994; Ryser \& Eek 2014; Reich 2014). Phenotypic plasticity to resource availability may be of key importance in introduced plants, when suddenly exposed to novel environmental conditions (Funk 2008; Davidson et al. 2011; Gratani 2014). In this study I test for a difference in plasticity between native and introduced populations, across separate treatments of nutrient and water availability.

Research that examines a change in phenotypic plasticity upon introduction to a new range often involves comparing a set of populations from the native range with a set of populations 
from the introduced alien range; using common gardens or controlled glasshouse conditions (Bossdorf et al. 2008; Zou et al. 2009). However, as populations can vary along geographic clines this can potentially obscure evolutionary differences between ranges (Colautti et al. 2009). To minimise unwanted noise caused by this, it is preferable to identify and make comparisons with the specific native population that was the source of the introduction. Genetic sequencing techniques, such as microsatellite markers, can be used to assess levels of genetic diversity from both within and between ranges, in addition to identifying the most likely source population of the introduction to the new range (Rollins et al. 2011). Through identifying the source population in this way, a more direct and robust comparison can be made to examine the change a species has undergone subsequent to being introduced.

Brandenburger et al. (2017) recently found introduced populations of the beach daisy, Arctotheca populifolia, to have longer stems and reduced leaf area and leaf shape complexity, when grown in controlled glasshouse conditions. Trait differences were tested with a set of populations from the introduced range, against a single population from the native range, identified as being the most likely source of the introduction (Rollins et al. 2011). In this study, I aim to test whether there is a difference in plasticity between the introduced Australian populations of $A$. populifolia and the source population of their introduction from the native range in South Africa. In accordance with general trends regarding greater plasticity in introduced species (Kaufman \& Smouse 2001; Richards et al. 2006; Hyldgaard \& Brix 2012), I predicted a shift towards increased plasticity in key growth-related traits in the introduced Australian range. 


\section{$\underline{5.2 \text { Methods }}$}

\subsubsection{Study species}

Arctotheca populifolia (Asteraceae) is a herbaceous succulent that can be either annual or a short-lived perennial. It is native to coastal areas of South Africa and is introduced to Australia, with its first presence recorded in the 1930's (AVH Database 2014); where it is now classed as an environmental and agricultural weed (Randall 2012). Since being introduced to Australia, A. populifolia has shown evidence of rapid evolution for an increase in plant height (Buswell et al. 2011). In a follow-up study, microsatellite markers were used to show that changes occurred despite very low levels of genetic diversity being retained in the introduced range of $A$. populifolia (Rollins et al. 2011). They also identified the locality of South African population that was the most likely source for the introduction of the species to the East coast of Australia (Arniston Beach (A); Figure 16). As I was interested in examining the potential evolutionary change in A. populifolia since its introduction, this was the sole population used from the native range. Four replicate Australian sites along the coast of New South Wales were used (Table S47), spanning a latitudinal gradient in temperature and rainfall. These sites comprised: Lighthouse beach (L), Wairo Beach (W), Narooma beach (N) and Mallacoota beach (M). Seeds were collected from the field for the one South African population in April 2011, and four Australian populations in February 2011. They were then grown through one generation in controlled glasshouse conditions (Brandenburger et al. 2017), to account for any maternal effects, prior to starting the experiment. 

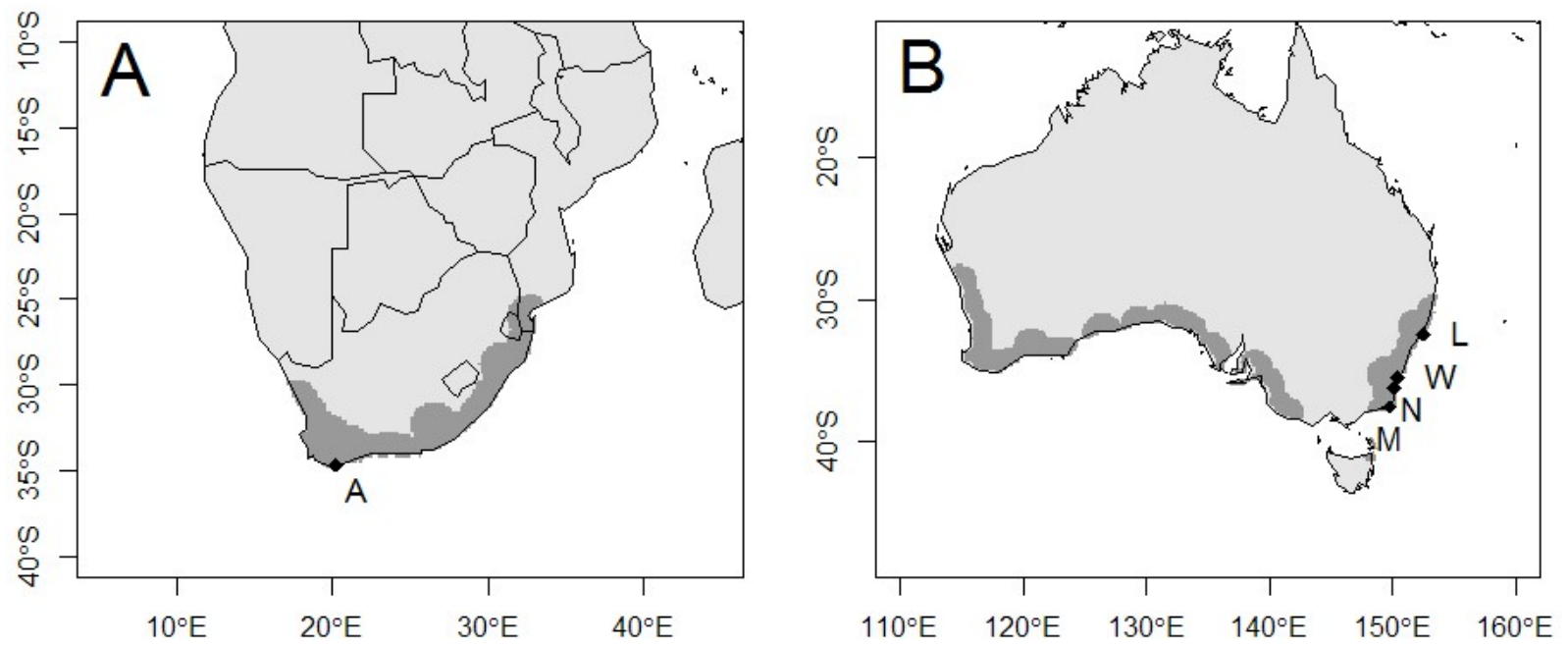

Figure 16. The study site locations in the native South African range (panel A) and the introduced Australian range (panel B). Each lettered point represents the corresponding study site for beaches at; Arnistoun (A), Lighthouse (L), Wairo (W), Narooma (N) and Mallacoota (M). Shaded dark grey areas show the known distribution of $A$. populifolia.

\subsubsection{Experimental design}

From November to May 2015, seeds of $A$. populifolia from native and introduced populations were germinated and grown in a glasshouse at the School of Biological, Earth and Environmental Sciences, UNSW Australia, Sydney, Australia,. Temperature in the glasshouse was controlled between $18-24^{\circ} \mathrm{C}$. Water was added to seeds in petri dishes sealed with parafilm in November 2014, stimulating germination over a four-week period. Following seed germination, half siblings were planted systematically in pairs from a maternal line, with one seed assigned to a high treatment pot and the other to a low treatment pot. The soil mix for pots consisted of 50\% river sand and 50\% cocopeat (Brandenburger et al. 2017). Pots in both experiments were rearranged in blocks across benches every two weeks to mitigate any positional effects on growth. Two experiments were run simultaneously, encompassing environmental treatments for: i) high and low nutrient availability, and ii) high and low water availability. 
In the nutrient treatment experiment, $65 \mathrm{~g}$ of general fertiliser and $65 \mathrm{~g}$ Osmocote slow-release, low phosphate fertiliser per $50 \mathrm{~L}$ of soil was added to the high nutrient treatment (see appendix for details on nutrient mix). The low nutrient treatment was supplied with $10 \%$ of the same nutrients (6.5g general fertiliser and $6.5 \mathrm{~g}$ Osmocote slow release fertiliser per $50 \mathrm{~L}$ of soil). Thirteen successfully germinated seedling pairs (paired for each maternal line) were planted into the high a low treatment pots for the South African population. Between 11 and 15 successfully germinated maternal pairs of seedlings were planted into each of the high and low treatment pots for the four Australian populations. A total of 130 plants were grown in the nutrient treatment experiment. Plants in the nutrient experiment were watered via sprinklers for 2 minutes twice a day, in order to maintain a constant supply of water.

Only two of the introduced populations were used for water treatment experiment, comprising the sites experiencing the highest $(\mathrm{L})$ and lowest $(\mathrm{M})$ levels of annual precipitation. Twelve successfully germinated maternal pairs of seedlings were planted into high and low water treatment pots for the South African population. Eleven maternal pairs of seedlings were planted in each treatment for both Australia populations. Seeds were planted in pots, with addition of $65 \mathrm{~g}$ of general fertiliser and $65 \mathrm{~g}$ Osmocote slow-release, low phosphate fertiliser per $50 \mathrm{~L}$ of soil mix (i.e., the same as the high nutrient treatment). All of the planted seeds were initially watered via sprinkler for two minutes twice a day until they became established, showing their first set of true leaves. Once established, the water treatment was initiated for each plant. High water treatment pots were manually watered at midday with $250 \mathrm{mls}$ of water every 2 days. To avoid sudden drought stress to the plants in the low water treatment, the application of water was gradually decreased after sprinkler watering was ceased. Low water treatment pots were watered at the same time, starting with $150 \mathrm{mls}$ every two days; this was reduced by $50 \mathrm{mls}$ each week until $50 \mathrm{mls}$ was added every two days. 


\subsubsection{Trait measurements}

A first set of measurements were made on each plant at 10 weeks post-germination, before the plants started flowering (Figure 17). These traits included height, leaf area, leaf shape, specific leaf area (SLA), chlorophyll fluorescence $\left(F_{v} / F_{M}\right)$ and isotope lab analyses of leaf chemical properties. Plant height was measured as the maximum 'stretched length' of the longest stem, using a piece of string from the base of the stem to the tip of the furthest fully expanded leaf as in Pérez-Harguindeguy et al. (2013). Leaf traits were collected from the third youngest fully-expanded leaf from each plant, which were removed, including the petiole and placed into a vial of de-ionised water. Leaves were scanned using a flatbed scanner, and their shape and area were calculated using imageJ (Schneider et al. 2012). Leaves were then oven dried at $60^{\circ} \mathrm{C}$ for 48 hours and subsequently weighed using a microbalance to determine oven dry weight. Leaf area (LA) was calculated as the total area of the leaf lamina. Leaf shape was calculated as the dissection index; the ratio of the perimeter of the leaf lamina divided by the square root of its area (McLellan \& Endler 1998). Specific leaf area (SLA; $\mathrm{mm}^{2} \mathrm{mg}^{-1}$ ) was calculated as the one-sided area of a fresh leaf $\left(\mathrm{mm}^{2}\right)$ divided by its oven dried mass $(\mathrm{mg})$, following Perez- Harguindeguy et al. (2013). Specific leaf area was measured including the petiole, as recommended by Cornelissen et al. (2003), to represent the entire contribution of the plant to its photosynthetic structure. A subset of leaves $(n=88)$ were then sent for analyses (on a Delta V, Thermo Finnigan Isotope Ratio Mass Spectrometer at the University of Sydney) to calculate leaf nitrogen content (percentage of dry matter; $N$ ) and carbon isotope $\delta^{13} \mathrm{C}$, as a measure of intrinsic water use efficiency (WUE; Seibt et al. 2008).

Measurements of leaf chlorophyll fluorescence were made, prior to removing leaves for above analysis, using a Junior-PAM, Walz, Germany. Leaf chlorophyll fluorescence can be used as predictor of plant fitness (Molina-Montenegro et al. 2013). The third youngest leaf on each plant was dark acclimated for one hour by wrapping it in foil, prior to measurements being taken. The fluorescence of a dark acclimated leaf was measured to assess the fraction of absorbed photons that are used for photochemistry $\left(F_{v} / F_{m}\right)$, calculated as $\left(F_{\mathrm{m}}-F_{\mathrm{o}} / F_{\mathrm{m}}\right)$ where 
$F_{m}$ is the fluorescence of a dark-adapted leaf during a saturating light flash and $F_{o}$ is the leaf fluorescence in total darkness. Dark adapted values of $F_{v} / F_{m}$ are around 0.8 for healthy plants, and can be used as an indicator of plant photosynthetic performance (Cavender-Bares \& Bazzaz 2004). Values lower than this are typically observed when a plant is under stress, indicating photoinhibition (Maxwell \& Johnson 2000).

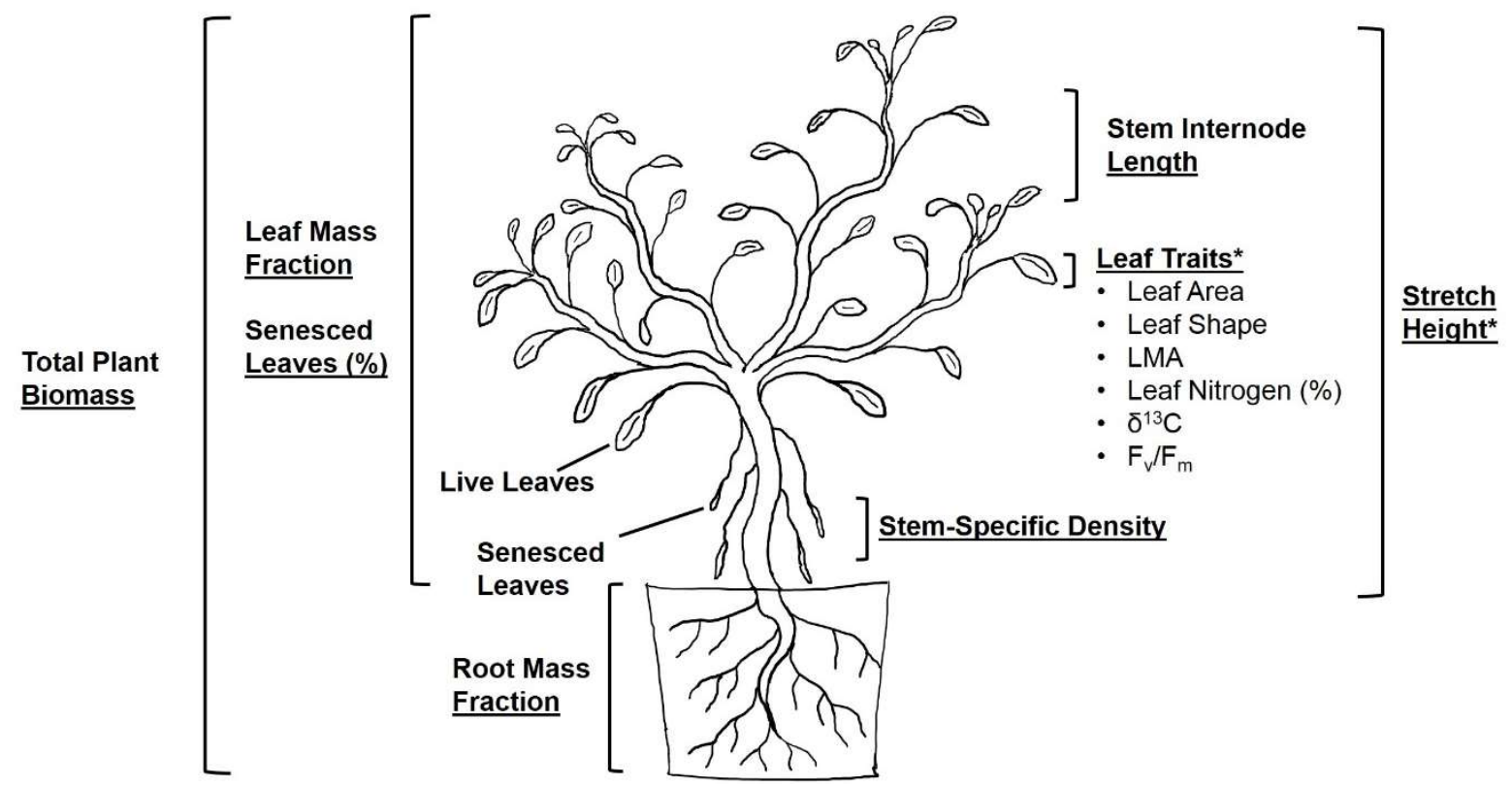

Figure 17. The trait measurements made on Arctotheca populifolia, relative to the scale at which they were measured on the plant. LMA; Leaf mass per Area.

*Indicates that traits were collected at 10 weeks after germinating. All other traits were collected on harvesting of plants at end of the experiment (22 weeks for nutrient treatment experiment; 25 weeks for water treatment experiment). 
Plants were harvested for biomass measurements at the end of the experiment, 22 weeks after germinating for the nutrient experiment and 25 weeks after germinating for the water treatment experiment. Traits collected at this time included stem internode length, stemspecific density (SSD), proportion of senesced leaves, and biomass allocation measurements. Upon harvest, each plant was divided into six parts; roots, stem (stem and branches), leaves (lamina and petiole), reproductive structures (flowers and peduncles), seeds, and a $10 \mathrm{~cm}$ long stem segment taken from the base of the stem. Roots were separated from the bulk of the soil using a sieve, and then carefully washed to minimise the loss of fine roots. Each plant part was placed in a paper bag and oven dried at $60^{\circ} \mathrm{C}$ for 3 days; on removal they were placed in a desiccating chamber for at least 30 minutes and then weighed on a micro-balance to determine oven dry weight.

The oven dry weight of all parts were pooled to give the total biomass of each plant, and three biomass allocation ratios. These were the root mass fraction (RMF; root mass per total plant biomass), leaf mass fraction (LMF; leaf mass per total plant biomass), and stem mass fraction (SMF; stem mass per total plant biomass). Biomass ratios were used, as they provide an easily interpreted number bounded between 0 and 1, and they are less sensitive to small changes in root to shoot ratios (Poorter \& Nagel 2000). Reproductive parts were collected and oven dried in order to contribute to the measure for total plant biomass. Reproductive mass fraction and other reproductive traits were excluded from the analysis because there was a large delay in the phenology of the South African plants flowering, in addition to no plants flowering at all in the low nutrient treatment. Stem-specific density (SSD; $\mathrm{mg} \mathrm{mm}^{-3}$ ) was measured from the $10 \mathrm{~cm}$ long basal stem segment. Stem-specific density is the oven dry mass of a section from the main stem of a plant $(\mathrm{mg})$ divided by the fresh volume of that section $\left(\mathrm{mm}^{3}\right)$; it is used synonymously with stem density and is an important characteristic of plant stability, defence, carbon gain and growth potential (Perez-Harguindeguy et al. 2013). The total number of living and senesced leaves of each plant was also counted on harvest. 
From these values I calculated the percentage of leaves that were senesced per plant (senesced leaves / total leaf number $\times 100$ ).

\subsubsection{Statistical Analyses}

All size related traits were $\log _{10}$ transformed to represent a proportional change in the size of that trait; this included leaf area, stretch height, stem internode length and total plant biomass. I used a linear mixed effects model (LME) to explain variation in each trait as a function of fixed effects for range (native vs. introduced), treatment (high vs. low), and their interaction, with a random term for population (in the Australian range). Sum of squares for the model were calculated using a type III Anova. Differences in phenotypic plasticity between ranges were determined by a significant interaction of treatment $\times$ range (Valladares et al. 2006).

Meta-analysis was used to summarise the overall difference in multi-trait plasticity between the introduced and native range, as an adapted version of the approach used in Davidson et al. (2011). The meta-analysis was carried out separately for the two environmental treatments. The slope of a reaction norm between high and low treatments was used as the index of plasticity (for each range). This is similar to Hedges' D, a commonly used effect size in metaanalyses, calculated as the difference between two means. Rather than simply calculating the differences between the high and low treatment values, I preferentially used the Beta coefficient of the treatment effect from a LME model to quantify the plasticity of each trait independently for the native range $\left(P_{n}\right)$ and introduced range $\left(P_{i}\right)$. Each trait was explained by one fixed term for treatment, plus a random term for population (Table S49).

In order to make plasticity comparable between different traits, I standardised the dataset prior to analysis by dividing each trait value by its pooled standard deviation across the South African and Australian populations. The pooled standard deviation was first calculated separately for each range (pooling the standard deviation of each treatment and populations), and then pooled across ranges. The pooled standard deviation was calculated as: 


$$
S D_{\text {pooled }}=\sqrt{\frac{\sum_{i=1}^{n} S D_{i}^{2}}{n}}
$$

Where $S D_{i}$ is the standard deviation for each group, and $n$ is the number of groups.

This is a modified way of calculating the effect size Hedges' $d$ (i.e., the standardised mean difference). By standardising using the standard deviation pooled between ranges, rather than standardising each range separately, it means that the effect size for each range will not be biased by different standard deviations in each range (this issue is discussed further in Chapter 6). The approach to summarise the overall difference in plasticity between the native and introduced ranges is similar to that used in Davidson et al. (2011), with an effect size calculated as the difference between two standardised beta-coefficients $\left(P_{\text {diff }}=P_{i}-P_{n}\right)$. Negative Beta-coefficients $\left(P_{i}, P_{n}\right)$ were converted to positive values prior to this; as for the purpose of this analysis I was interested in the absolute difference in plasticity, not the direction.

Meta-analysis was carried out using a random effects model to give a mean overall effect size. Each trait was weighted in the model according to the inverse of its variance. The variance $\left(V_{B}\right)$ of the difference between two uncorrelated effect sizes is calculated simply as the sum of their variances (Borenstein et al. 2009a). A value of $P_{\text {diff }}>0$ would indicate that the introduced populations showed a greater degree of phenotypic plasticity than the native population. Standardising in this way changes the units to the pooled standard deviation for that trait (i.e., an effect size of one would mean that the Australian populations had greater plasticity by one unit of pooled standard deviation). Statistical analyses were carried out in R v.3.0.1 (R Core Team 2013). 


\section{$\underline{5.3 \text { Results }}$}

\subsubsection{Nutrient Experiment}

There was a significant difference in plasticity between the South African and Australian ranges for four out of thirteen traits in the nutrient treatment experiment (indicated by a significant range $\times$ treatment interaction). Leaf shape, leaf area, stretch height, and root mass fraction all showed significantly lower plasticity in the introduced Australian populations (Figure 18; Table S48). The South African population showed significantly greater plasticity in leaf area $(B=0.285, p=0.032)$, through a greater increase in leaf area when nutrients were not limiting. The Australian plants had simple ovate-shaped leaves that showed no plasticity across treatments. In contrast, the South African plants had far more complex, lobed leaves when grown in the high nutrient treatment, but only grew simple ovate leaves when nutrients were limiting, and thus exhibited much greater plasticity in leaf shape complexity $(B=1.296$, $p<0.001)$. The South African and Australian plants both had very similar stretch stem length in the high nutrient treatment. However, when nutrients were limiting the Australian plants showed much less of a reduction in stretched stem length, and thus significantly lower plasticity $(B=-0.351, p<0.001)$. The Australian populations showed reduced plasticity in root mass fraction through less of an increase in allocation to root biomass when nutrients were limiting $(B=0.021, p=0.031)$. 

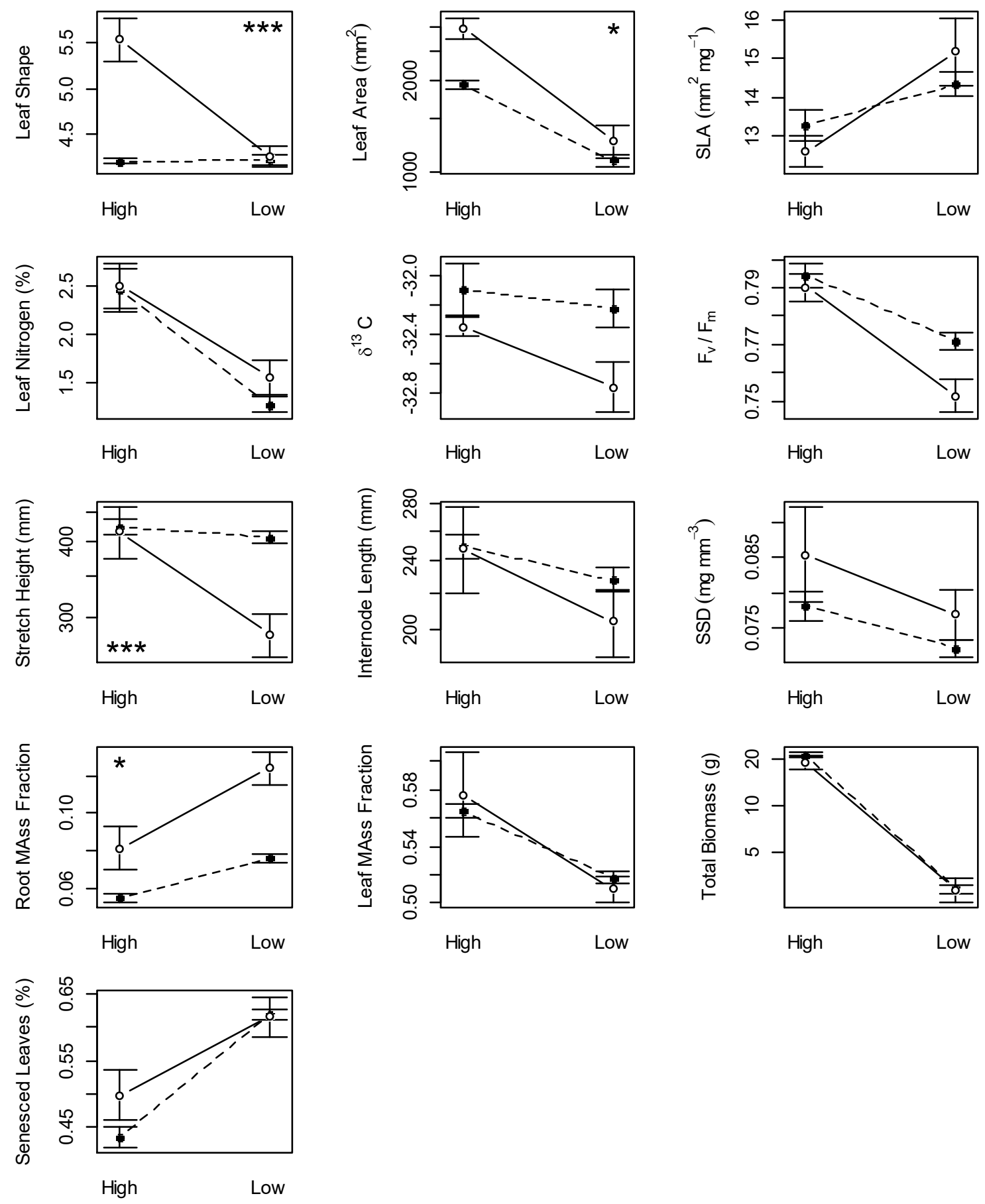

Figure 18. Reaction norms for the South African and Australian populations across high and low nutrient treatments. Open circles ( $(\circ)$ represent the native South African population, closed circles $(\bullet)$ represent the pooled Australian populations. Significant differences in phenotypic plasticity (determined by a significant interaction of range $\mathrm{x}$ treatment) are represented with asterisks $\left(p<0.05={ }^{* *}, p<0.01=\right.$ “**’, $\left.p<0.001=^{(* * *)}\right)$. Error bars denote standard error. Acronyms: SLA, specific leaf area; SSD, stem-specific density. 


\subsubsection{Water Experiment}

There was a significant difference in plasticity between the South African and Australian ranges for three out of thirteen traits across water treatments (indicated by a significant range $\times$ treatment interaction). The introduced Australian population had significantly lower plasticity in leaf mass fraction, stem internode length, and percentage of senesced leaves (Figure 19; Table S49). The Australian population showed significantly lower plasticity in $\operatorname{LMF}(B=0.02$, $p=0.036)$, by exhibiting less of an increase in allocation to leaf biomass when water was limiting. Both the South African and Australian populations showed a similar level of allocation to leaf biomass in the high water treatment, but the Australian population showed reduced plasticity though a diminished increase in LMF when water was limiting $(B=0.02, p=0.036)$. Plasticity of stem internode length was significantly greater in the South African population ( $B$ $=-8.9, p=0.003)$, which showed a greater increase in internode length in the high water treatment. The Australian plants showed no difference in the percentage of senesced leaves between water availability treatments, whereas the South African plants displayed significantly greater plasticity $(B=11.55, p<0.001)$, with a much higher proportion of leaves senesced when water was limiting. 

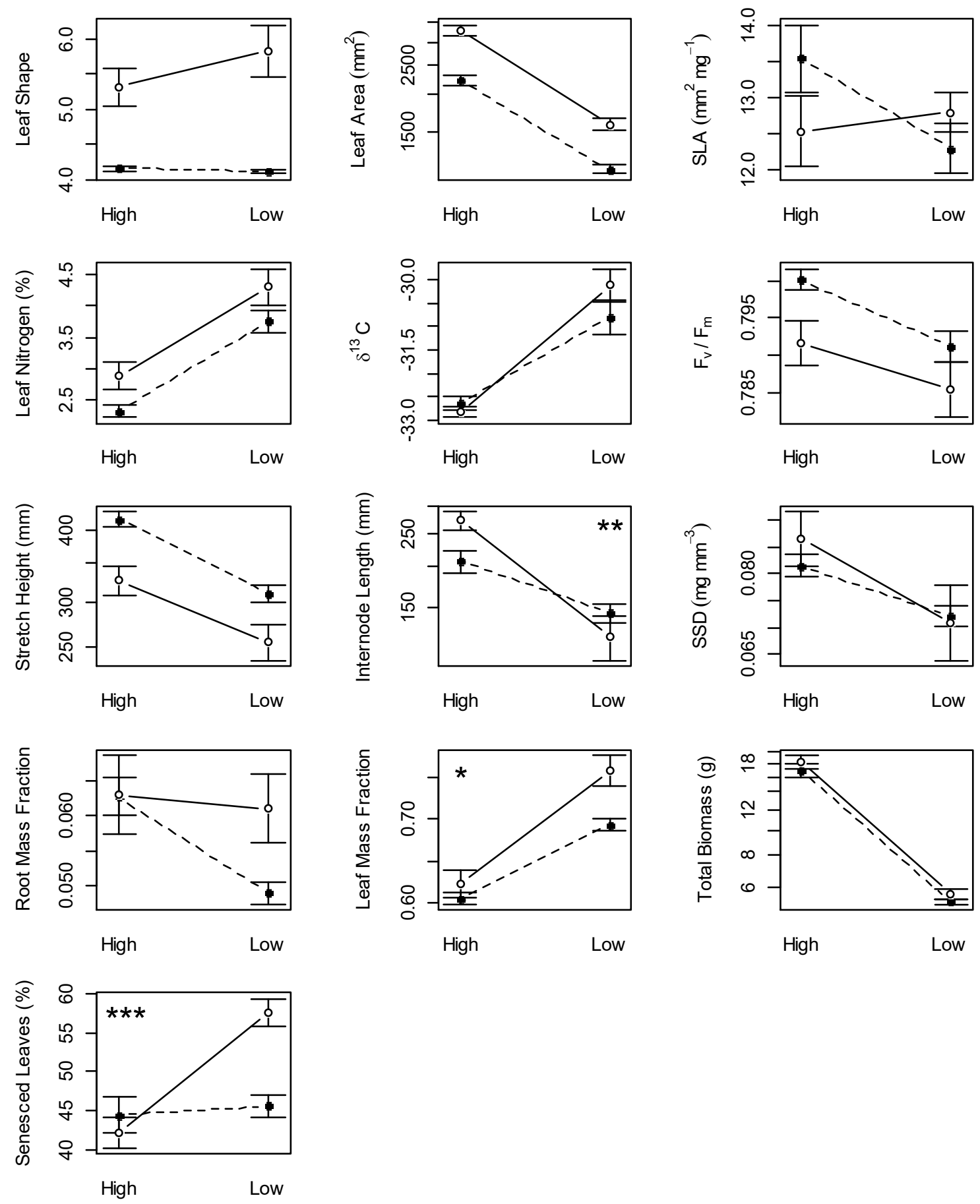

Figure 19. Reaction norms for the South African and Australian populations across high and low water availability treatments. Open circles (०) represent the native South African population, closed circles $(\bullet)$ represent the pooled Australian populations. Significant differences in phenotypic plasticity (determined by a significant interaction of range $x$ treatment) are represented with asterisks $\left(p<0.05={ }^{* *}, p<0.01={ }^{* * *}, p<0.001={ }^{* * * *}\right)$. Error bars denote standard error. Acronyms: SLA, specific leaf area; SSD, stem-specific density. 


\subsubsection{Overall differences in phenotypic plasticity}

The majority of traits were more plastic in the native South African population in response to the nutrient treatment (Figure 20). Using a random effects meta-analysis model to combine all traits into an overall effect size, I found that the introduced Australian populations displayed significantly lower mean plasticity than the native South African population in response to nutrient treatment (Mean $P_{\text {diff }}=-0.55$ [95\% Cl: -0.98 to -0.13$]$ ). Differences in plasticity between the native and introduced populations were more mixed in the water treatment experiment. The Australian population showed lower mean plasticity, though this difference was not statistically significant (Mean $P_{\text {diff }}=-0.37[95 \% \mathrm{Cl}:-0.82$ to 0.08$]$ ). 

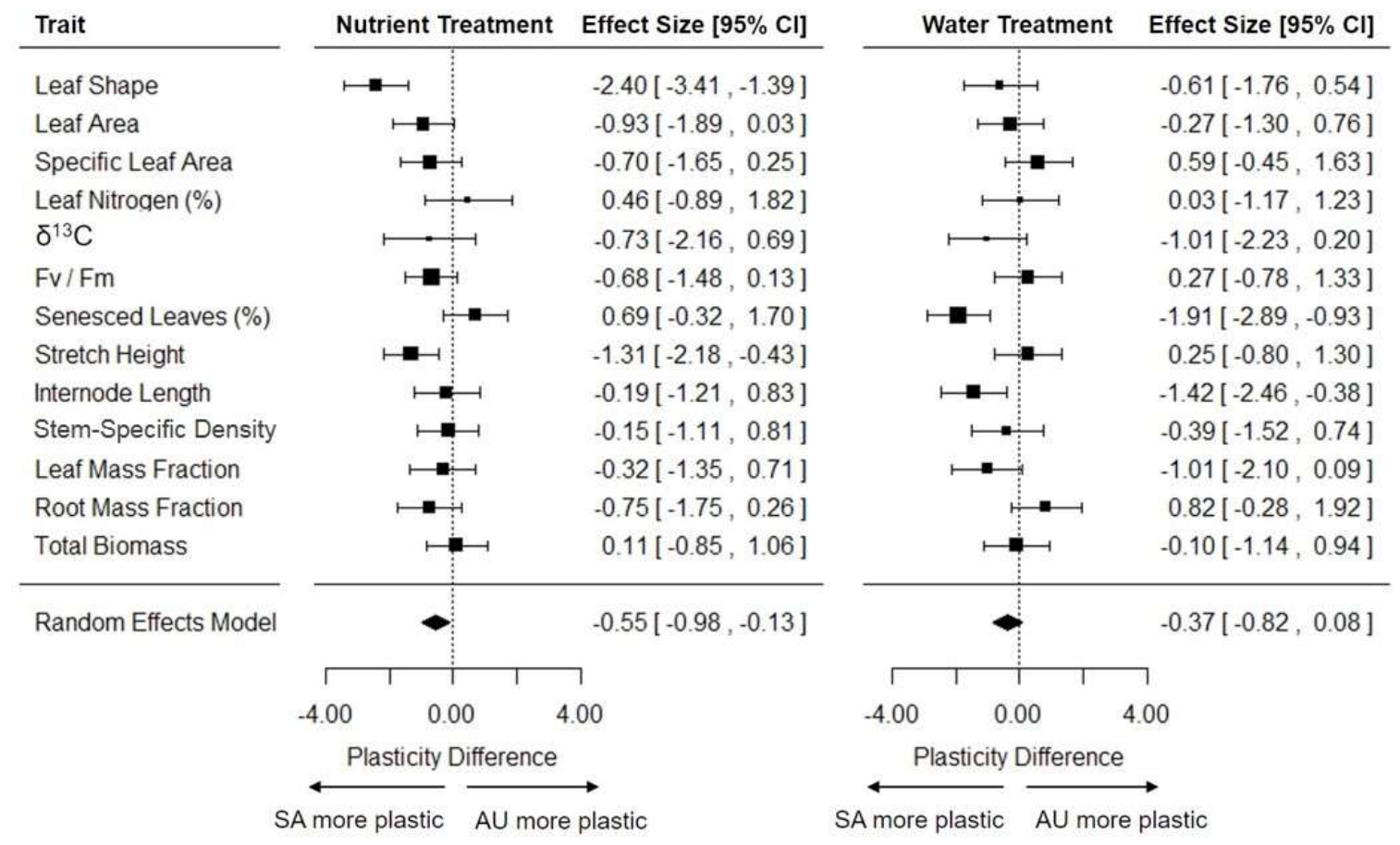

Figure 20. The overall difference in plasticity between the South African (SA) population and the introduced Australian (AU) populations in the nutrient and water treatment experiments. The effect size (the difference in plasticity between ranges) was calculated as reaction norm slope of the introduced populations minus the reaction norm slope of the native population $\left(P_{\text {diff }}=P_{i}-P_{n}\right)$. A positive effect size signifies that the introduced populations had greater plasticity for that trait than the native population. Square centres indicate the effect size, and bar width indicate their $95 \% \mathrm{Cl}$. Square size indicates the weighting of that trait in the model, based on an inverse of the variance, larger squares contribute more weight to the model. The diamond represents the overall effect size for all traits, calculated from a meta-analyis using a random effects model. 


\section{$\underline{5.4 \text { Discussion }}$}

Introduced populations of Arctotheca populifolia showed lower plasticity in response to variation in nutrient and water availability than their native source population. This change has occurred in as little as 80 years since their presence was first documented in Australia. Other studies comparing phenotypic plasticity between native and introduced populations have found opposing results to this study (Lavergne \& Molofsky 2007; Cano et al. 2008; van Kleunen and Fischer 2008). Hyldgaard \& Brix (2012) found higher levels of phenotypic plasticity in fitness-related traits in response to temperature in the invasive populations of Ceratophyllum demersum. Similarly, Melaleuca quinquenervia, an invasive wetland tree to Florida, was found to have greater phenotypic plasticity in response to varying water and $\mathrm{pH}$ levels than populations from its native range (Kaufman \& Smouse 2001). Bossdorf et al. (2008) found introduced populations of Senecio inaequidens to show an increase in plasticity in response to nutrient availability, interpreting this as the selective introduction of more plastic pre-adapted genotypes to the introduced range.

Flory et al. (2011) found no difference in plasticity between native and introduced populations of Microstegium vinimeium across a range of different environments. Despite this, the introduced populations still had greater biomass, survival, and outperformed the natives in all garden environments, indicating that greater plasticity was not necessary to grow successfully across a range of environmental conditions. Introduced populations of the tree Acer negundo, also showed greater plant biomass relative to their native conspecifics, but no difference in plasticity to nutrient availability (Lamarque et al. 2013). Although the loss of plasticity in introduced populations of $A$. populifolia observed in this study is counter to my hypotheses and general patterns in the literature, there are a number of factors that could drive this change in plasticity in the introduced range, which I will now outline. 


\subsubsection{Costs and limits to plasticity}

While phenotypic plasticity can provide a fitness benefit for individuals and populations (Sultan \& Bazzaz 1993; Whitlock 1996; Sultan 2001; Richards et al. 2006), it is also associated with a number of costs and limitations (DeWitt et al. 1998). A cost to plasticity occurs in maintaining the genetic and cellular machinery necessary to be plastic (Scheiner 1993), and can cause a decrease in fitness even when an optimal phenotype is expressed (Pigliucci 2005). Thus, plasticity may be selected against if it does not provide a net fitness benefit in the introduced range, relative to a less plastic genotype.

A plastic response that has been selected to be advantageous in one environment, may not always transpire to be beneficial in novel environmental conditions. This results in limits to plasticity, which occur when a plastic response produces a sub-optimal trait in relation to its environment (DeWitt et al. 1998; Pigliucci 2005). This can transpire if an organism cannot reliably detect environmental cues to express an optimal phenotype in a new environment (Moran 1992; Getty 1996; Visser et al. 2008), or if there is a lag-time in sensing and responding to an environmental cue in order to produce the optimal phenotype at the right time (Moran 1992; Stomp et al. 2008). Thus, if the Australian environment was less predictable or differed sufficiently in its environmental cues so that plasticity did not provide a net fitness benefit, then plasticity may have been selected against and rapidly declined in the introduced populations of $A$. populifolia.

\subsubsection{Plasticity may not be beneficial in the introduced range environment}

Phenotypic plasticity could be selected against if it is not advantageous in the introduced range environment (Alpert \& Simms 2002). If there was a decrease in resource availability in Australian sites, then lower plasticity may have been selected for in response to resource availability in two different ways. Firstly, low resource environments are thought to favour reduced plasticity, and thus it may be directly selected against in favour of more canalised phenotypes (Crick \& Grime 1987; Emery 1994; Grassein et al. 2010). Secondly, a decline in 
plasticity can arise indirectly if introduced populations become more specialised to a low resource environment (Taylor \& Aarssen 1988; Lortie \& Aarsen 1996). The 'specialisation hypothesis' (Lortie \& Aarsen 1996) proposes that ecotypes specialised to more favourable environmental conditions should show an increase in plasticity, while those specialised to less favourable conditions should show a decrease in plasticity. Thus, the relatively low plasticity of some traits (e.g., height) in the Australian populations could be due to adaptation to perform better in low resource conditions, resulting in flatter reaction norms (cf. van Kleunen et al. 2005).

\subsubsection{Shift to 'jack-of-all trades' strategy}

A loss of plasticity in the Australian populations could have occurred if they underwent a shift to more of a 'jack-of-all trades' strategy (Richards et al. 2006 [i.e., a general purpose genotype; Baker 1965; Grime 1977]). The 'jack-of-all-trades' strategy describes a scenario in which plasticity in fitness traits is reduced, so that individuals or populations can maintain more constant fitness across variable environments (Richards et al. 2006). Chlorophyll fluorescence $\left(F_{v} / F_{m}\right)$, is an indicator of plant health in stressful environments and can be used as a proxy of plant fitness (Maxwell \& Johnson 2000; Molina-Monenegro et al. 2013). $F_{v} / F_{m}$, was generally higher in the Australian populations, particularly in the low nutrient treatment, indicating that the introduced populations were performing better in these conditions. However, plant biomass, another key fitness-related trait that integrates many proximate physiological and morphological responses (Voille et al. 2007), showed no variation in plasticity or mean trait value in treatments between ranges. This suggests that while there is an apparent reduction in plasticity in the introduced range, this may not be having a major impact on overall plant performance (at least in terms of its vegetative biomass). 


\subsubsection{Genetic assimilation}

It is possible that plasticity is only beneficial in the early stages of an invasion, and is then selected against once the new population is established. Plasticity can be beneficial in the colonisation of new environments (Yeh \& Price 2004; Ghalambor et al. 2007; Lande 2015). However, it may then be selected against post-introduction if it is no longer necessary, in a process known as genetic assimilation (Waddington 1953; Pigliucci 2006). High levels of plasticity present in the native source population can help colonising plants to persist when introduced to new range, through adjusting their phenotype to better match the new environment. This may lead to a temporary selection for an increase in plasticity in the introduced population (Lande 2009). Once the new optimal phenotype has been reached and plasticity is no longer necessary, it is then selected against, resulting in an overall reduction in plasticity (Lande et al. 2009; Pfennig et al. 2010; Lande 2015). This could be one reason why there is a shift in mean trait values, along with a decline in plasticity in the introduced populations of $A$. populifolia. Although this process has been predicted theoretically (Lande 2009; Crispo et al. 2010; Gomez-Mestre \& Giovanni 2013; Lande 2015), it is very difficult to prove empirically without tracking the change in plasticity through time.

\subsubsection{Founder effects}

A final possibility is that differences in plasticity between ranges may have arisen due to founder effects, rather than being directly selected for. Small population sizes, low genetic variance, and founder effects have been suggested to contribute to reduced plasticity in populations on range margins (Magi et al. 2011). When introduced to a new geographic range, colonising species with small populations can experience genetic drift or population bottlenecks as a consequence of founder effects, which can lead to a set of distinctly different traits than those present in native range populations (Mayr 1942; Eckert et al. 1996; Mutate 2013). When combined with reproductive isolation and novel selection pressures in the new 
range, founder effects can result in the rapid evolution of divergent populations with different characteristics from their parent population (Mayr 1954; Dlugosch \& Parker 2008).

Williams et al. (2008) concluded that founder effects were the most likely explanation for an increase in plasticity to nutrient availability in introduced populations of Cynoglossum officianale, given the low levels of genetic diversity within and between introduced populations compared to those from the native range. Using microsatellite markers, Rollins et al. (2011) found that the introduced populations of $A$. populifolia in this study retained only $1 \%$ of their native range genetic diversity, in addition to having little between-population diversity in Australia. Consequently, it is plausible that founder effects may have had a strong influence on the introduced populations of $A$. populifolia, and may be a sufficient explanation for the reduced levels of plasticity they express. Interestingly, A. populifolia has become successful in its introduced Australian range despite showing both low levels of genetic diversity and a decline in phenotypic plasticity. 


\section{$\underline{5.5 \text { Summary }}$}

Contrary to previously documented patterns in plants (Kaufman \& Smouse 2001; Bossdorf et al. 2008; Cano et al. 2008), plasticity did not increase in the introduced range of $A$. populifolia, but actually showed an overall decline in as little as 80 years since being introduced. I have outlined a number of reasons why this may be the case, including specialisation to the Australian environment, the selection against plasticity if it is not beneficial in the introduced range, a shift to a jack of all trades strategy, genetic assimilation and/or founder effects. The divergent levels of plasticity observed between ranges could have been due to a combination of these factors. A greater understanding of the environmental conditions present in each range that may select for or against plasticity, in addition to more robust measures of fitness (e.g., reproductive output), would be beneficial to better understand the adaptive nature of this loss plasticity in the introduced range. This is the first study to show a rapid decline in phenotypic plasticity over such a short timescale in populations of an introduced plant, relative to their native source population. 


\subsection{Appendix C - Supplementary Material}

Table S47. Location of the sample sites for Arcotheca populifolia.

\begin{tabular}{llccr}
\hline Country & Site name & Population & Latitude & Longitude \\
\hline South Africa & Arniston beach & A & -34.6579 & 20.2329 \\
Australia & Lighthouse beach & L & -32.4468 & 152.2511 \\
Australia & Mallacoota beach & M & -37.5688 & 149.7621 \\
Australia & Narooma beach & N & -36.2238 & 150.1401 \\
Australia & Wairo beach & W & -35.4423 & 150.4088 \\
\hline
\end{tabular}


Table S48. The difference in phenotypic plasticity between the Australian and South African Ranges, in response to both the nutrient and water experiments. A significant difference in phenotypic plasticity between ranges is determined by a Treatment $x$ Range interaction in two-way Anova LME model. Significant differences in plasticity are highlighted with asterisks $\left(p<0.1=?, p<0.05={ }^{\prime *}, p<0.01={ }^{\prime * *}, p<0.001={ }^{* * * * \prime}\right)$. Acronyms: SLA, specific leaf area; WUE, water-use efficiency; SSD, stem-specific density; RMF, root mass fraction; LMF, leaf mass fraction.

\begin{tabular}{|c|c|c|c|c|c|c|c|c|c|c|c|c|c|c|}
\hline \multirow{3}{*}{ Trait } & \multicolumn{6}{|c|}{ Nutrient Treatment } & & \multicolumn{7}{|c|}{ Water Treatment } \\
\hline & \multicolumn{2}{|c|}{ Treatment } & \multicolumn{2}{|c|}{ Range } & \multicolumn{2}{|c|}{ Treatment $\times$ Range } & & \multicolumn{2}{|c|}{ Treatment } & \multicolumn{2}{|c|}{ Range } & \multicolumn{3}{|c|}{ Treatment $\times$ Range } \\
\hline & $B$ & $p$ & $B$ & $p$ & $B$ & $p$ & & $B$ & $p$ & $B$ & $p$ & $B$ & $p$ & \\
\hline Leaf Shape & 0.014 & 0.884 & 1.328 & $<0.001$ & -1.296 & $\leq 0.001$ & $* * *$ & -0.052 & $<0.001$ & 1.155 & 0.812 & 0.571 & 0.107 & \\
\hline Leaf Area ${ }^{\dagger}$ & -0.261 & $<0.001$ & 0.177 & $<0.001$ & -0.124 & $\underline{0.032}$ & * & -0.299 & $<0.001$ & 0.169 & $<0.001$ & -0.017 & 0.613 & \\
\hline $\operatorname{SLA}\left(\mathrm{mm}^{2} \mathrm{mg}^{-1}\right)$ & 1.023 & 0.024 & -0.727 & 0.667 & 1.556 & 0.102 & & -1.078 & 0.019 & -0.886 & 0.622 & 1.339 & 0.070 & . \\
\hline Leaf Nitrogen (\%) & -1.199 & $<0.001$ & 0.039 & 0.899 & 0.252 & 0.498 & & 1.413 & $<0.001$ & 0.562 & 0.049 & -0.018 & 0.964 & \\
\hline$\delta 13 C$ (WUE) & -0.129 & 0.490 & -0.254 & 0.568 & -0.280 & 0.32 & & -0.191 & $<0.001$ & 1.833 & 0.726 & 0.876 & 0.106 & \\
\hline $\mathrm{F}_{\mathrm{v}} / \mathrm{F}_{\mathrm{m}}$ & -0.023 & $<0.001$ & -0.004 & 0.574 & -0.015 & 0.159 & & -0.009 & 0.001 & -0.008 & 0.013 & 0.003 & 0.584 & \\
\hline $\begin{array}{l}\text { Senesced leaves } \\
(\%)\end{array}$ & 0.186 & $<0.001$ & 0.062 & 0.262 & -0.068 & 0.072 & . & 1.163 & 0.635 & -2.255 & 0.471 & 14.296 & $\leq 0.001$ & $* \star *$ \\
\hline Stretch Height ${ }^{\dagger}$ & -0.012 & 0.572 & -0.020 & 0.660 & -0.153 & $\leq 0.001$ & $* * *$ & -0.132 & $<0.001$ & -0.110 & $<0.001$ & 0.020 & 0.609 & \\
\hline Internode length ${ }^{\dagger}$ & -0.040 & 0.096 & -0.030 & 0.519 & -0.028 & 0.607 & & -0.145 & 0.005 & 0.142 & 0.025 & -0.249 & $\underline{0.005}$ & $* *$ \\
\hline $\mathrm{SSD}\left(\mathrm{mg} \mathrm{mm}^{-3}\right)$ & -0.006 & 0.032 & 0.007 & 0.096 & -0.002 & 0.690 & & -0.009 & 0.028 & 0.005 & 0.351 & -0.006 & 0.401 & \\
\hline RMF & 0.021 & $<0.001$ & 0.026 & $<0.001$ & 0.021 & $\underline{0.031}$ & * & -0.014 & $<0.001$ & 0.000 & 0.944 & 0.012 & 0.083 & . \\
\hline LMF & -0.047 & $<0.001$ & 0.011 & 0.423 & -0.016 & 0.328 & & 0.088 & $<0.001$ & 0.017 & 0.281 & 0.048 & $\underline{0.036}$ & * \\
\hline Total Biomass $^{\dagger}$ & -0.881 & $<0.001$ & -0.058 & 0.272 & 0.020 & 0.790 & & -0.491 & $<0.001$ & 0.036 & 0.504 & -0.008 & 0.85 & \\
\hline
\end{tabular}

${ }^{\dagger}$ Indicate size related traits were $\log _{10}$ transformed prior to analysis to represent proportional change in size. 
Table S49. The phenotypic plasticity for each range, in response to high and low nutrient and water treatments. Plasticity for each range is calculated as the slope of the treatment effect in a one way Anova LME model (Trait Treatment), for each trait with values that have been standardised by the pooled standard deviation between ranges. The standardised betacoefficient $(\beta)$ was the effect size used the meta-analysis. Acronyms: SLA, specific leaf area; WUE, water-use efficiency; SSD, stem-specific density; RMF, root mass fraction; LMF, leaf mass fraction.

\begin{tabular}{|c|c|c|c|c|c|c|c|c|}
\hline \multirow{3}{*}{ Trait } & \multicolumn{4}{|c|}{ Nutrient Treatment } & \multicolumn{4}{|c|}{ Water Treatment } \\
\hline & \multicolumn{2}{|c|}{ Australia } & \multicolumn{2}{|c|}{ South Africa } & \multicolumn{2}{|c|}{ Australia } & \multicolumn{2}{|c|}{ South Africa } \\
\hline & $\beta$ & $p$ & $\beta$ & $p$ & $\beta$ & $p$ & $\beta$ & $p$ \\
\hline Leaf Shape & 0.044 & 0.835 & -1.385 & $<0.001$ & -0.382 & 0.242 & 0.479 & 0.26 \\
\hline Leaf Area $^{\dagger}$ & -1.464 & $<0.001$ & -1.558 & $<0.001$ & -1.817 & $<0.001$ & -1.830 & $<0.001$ \\
\hline SLA & 0.430 & 0.019 & 0.935 & 0.016 & -0.594 & 0.027 & 0.195 & 0.651 \\
\hline Leaf Nitrogen (\%) & -1.436 & $<0.001$ & -1.196 & 0.005 & 1.606 & $<0.001$ & 1.306 & $<0.001$ \\
\hline ס13C (WUE) & -0.241 & 0.536 & -0.897 & 0.048 & 1.391 & $<0.001$ & 1.673 & $<0.001$ \\
\hline$F_{v} / F_{m}$ & -0.840 & $<0.001$ & -1.409 & $<0.001$ & -0.948 & 0.001 & -0.546 & 0.198 \\
\hline $\begin{array}{l}\text { Senesced leaves } \\
(\%)\end{array}$ & 1.502 & $<0.001$ & 0.884 & 0.024 & 0.132 & 0.671 & 1.564 & $<0.001$ \\
\hline Stretch Height ${ }^{\dagger}$ & -0.124 & 0.053 & -1.049 & 0.004 & -1.425 & $<0.001$ & -1.065 & 0.007 \\
\hline Internode length $^{\dagger}$ & -0.390 & 0.049 & 0.387 & 0.356 & -0.833 & $<0.005$ & -1.457 & $<0.001$ \\
\hline SSD & -0.479 & 0.016 & -0.435 & 0.276 & -0.914 & $<0.002$ & -0.721 & 0.091 \\
\hline RMF & -1.205 & $<0.001$ & -0.779 & 0.006 & 1.572 & $<0.001$ & 1.530 & 0.784 \\
\hline LMF & 1.390 & $<0.001$ & 0.337 & 0.044 & -1.269 & $<0.001$ & -1.477 & $<0.001$ \\
\hline Total Biomass $^{\dagger}$ & -1.819 & $<0.001$ & -1.676 & $<0.001$ & -1.752 & $<0.001$ & -1.879 & $<0.001$ \\
\hline
\end{tabular}

${ }^{\dagger}$ Indicate size related traits were $\log _{10}$ transformed prior to analysis to represent proportional change in size. 
Part III

Supporting Chapters 


\title{
6. An effect size of an effect size: a problem with calculating a difference in plasticity between two groups.
}

\begin{abstract}
:
Hedges' $d$ is a commonly used effect size in meta-analyses, calculated as the difference between two means, divided by their pooled standard deviation. The difference between two Hedges' ds can be calculated to examine the magnitude of difference in effect size between two groups. However, if the two groups have different standard deviations, then the calculation of Hedges' $d$ will result in them being standardised to different units. Consequently this can give unrealistic/non-representative effect sizes of their true difference in magnitude. I illustrate this using Hedges' $d$ to calculate phenotypic plasticity independently for native and introduced populations of Arctotheca populifolia, and then calculate the difference between them $\left(P_{\text {diff }} v 1\right)$. I then go on to propose an alternative method to calculate Hedges' $d$, when looking to calculate a difference in plasticity between two groups $\left(P_{\text {diff }} v 2\right)$. This method provides a greatly improved estimate of the true differences in plasticity (i.e., difference in reaction norm slopes) between the native and introduced populations.
\end{abstract}




\section{$\underline{6.1 \text { Rationale }}$}

In the previous chapter (Chapter 5), I used a meta-analysis approach to calculate an overall difference in phenotypic plasticity $\left(P_{\text {diff }}\right)$ between the native source population and introduced populations of Arctotheca populifolia. This approach was based on the method used in Davidson et al. (2011), to calculate a difference in plasticity between invasive and native/noninvasive species. Phenotypic plasticity can be expressed as a reaction norm, calculated as the difference between two means. Hedges' $d$ is an effect size, calculated as the difference between two means, divided by their pooled standard deviation (SD). It allows traits that are in different units (e.g., plant height [mm] and SLA $\left[\mathrm{mm}^{2} \mathrm{mg}^{-1}\right]$ ) to be included in the same metaanalysis, by converting them into units of pooled standard deviation.

Hedges' $D$ (not to be confused with Hedges's $d$; see Box 2) is a commonly used effect size, calculated as the difference between two means. Davidson et al. (2011) used Hedges' $D$ to calculate an effect size for the difference phenotypic plasticity $\left(P_{\text {diff }}\right)$ between invasive and native/non-invasive populations, as the difference between two Hedges' ds (i.e., an effect size of an effect size). They found that overall plasticity was significantly greater in the invasive populations. When calculating a difference between two Hedges' ds, it is assumed that the two groups being compared (i.e., the native and introduced populations) should have equal standard deviations. However, if the two groups have different standard deviations, then the calculation of Hedges' $d$ will result in them being standardised to different units. Thus, calculating $P_{\text {diff }}$ as a difference between these two effect sizes could give a result that does not truly reflect their real difference in plasticity.

In this chapter $\mathrm{I}: \mathrm{i}$ ) demonstrate the problem with calculating $P_{\text {diff }}$ (hereafter referred to as $P_{\left.\text {diff } \_v 1\right)}$ as a difference between two Hedges' $d s$ from groups that have non-equal standard

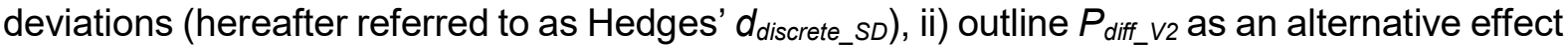
size, which calculates Hedges' $d$ while accounting for non-equal standard deviations between 
groups (hereafter referred to as Hedges' $d_{\text {combined_sD }}$ ), and iii) show the difference in the results that these two approaches have on real data.

\section{Box 2. Glossary of terms related to calculating an effect size:}

Hedges' $D$ : Effect size of the raw difference between two means $\left(D=M_{1}-M_{2}\right)$

Hedges' $d$ : Effect size of the standardised difference between two means $\left(d=\frac{M_{1-M_{2}}}{S D_{\text {pooled }}}\right)$

$\boldsymbol{P}_{\boldsymbol{I}}$ and $\boldsymbol{P}_{\boldsymbol{N}}$ : index for the plasticity of the native $\left(\boldsymbol{P}_{\boldsymbol{N}}\right)$ or introduced $\left(\boldsymbol{P}_{\boldsymbol{I}}\right)$ populations, calculated as Hedges' $d$ for that range

$\mathbf{P}_{\text {diff: }}$ the difference in plasticity $(\boldsymbol{P})$ between native and invasive populations.

If $\mathrm{P}_{\text {diff }}>0$ it means that the introduced plants are more plastic. $\left(P_{\text {diff }}=P_{I}-P_{N}\right)$

$S D_{\text {pooled: }}$ The pooled standard deviation for a set of $n$ groups, in this case groups of

populations in high and low treatments $\left(S D_{\text {pooled }}=\sqrt{\frac{\sum_{i=1}^{n} S D_{i}^{2}}{\sum n}}\right)$

$S D_{\text {combined: }}$ The combined mean $S D_{\text {pooled }}$ for two groups, used to calculate Hedges' $d$; in this case the native and introduced ranges $\left(S D_{\text {combined }}=\sqrt{\frac{S D_{\text {pooled_I }}^{2}+S_{\text {pooled_N }}^{2}}{2}}\right)$

\section{Introduced terms:}

Hedges' $\boldsymbol{d}_{\text {discrete_sD: }}$ the original method to calculate Hedges' $d$, described above.

Hedges' $\boldsymbol{d}_{\text {combined_sD: }}$ updated method to calculate Hedges' $d$, recommend to be used when calculating $P_{\text {diff }}\left(d=\frac{M_{1-M_{2}}}{S D_{\text {combined }}}\right)$

$\boldsymbol{P}_{\text {diff_v1: }}$ The original method to calculate $P_{\text {diff, described above. }}$

$\boldsymbol{P}_{\text {diff_v2: }}$ An updated method to calculate $P_{\text {diff, }}$ using Hedges' $d_{\text {combined_sD }}$ 


\subsection{Approach}

\subsubsection{Calculation of $P_{\text {diff_v1 }}$ from Hedges' $d_{\text {discrete_sD }}$}

Phenotypic plasticity for the native $\left(P_{N}\right)$ and introduced $\left(P_{I}\right)$ range is calculated using the effect size Hedges' $d$ (Davidson et al. 2011). That is, the difference between the maximum mean treatment value and the minimum mean treatment value, divided by the pooled standard deviation across both treatments $\left(S D_{I, N}\right)$. This converts the measure of plasticity into units of pooled standard deviation. $P_{\text {diff } v 1}$ can then be used to calculate an effect size for a difference in plasticity between two groups (i.e., native and introduced populations):

$$
P d i f f_{-} v 1=P_{I}-P_{N}=\frac{I_{\max }-I_{\min }}{S D_{I}}-\frac{N_{\max }-N_{\min }}{S D_{N}}
$$

Where: $\quad I_{\max }$ is the maximum treatment mean value for the introduced range

$I_{\min }$ is the minimum treatment mean value for the introduced range

$N_{\text {max }}$ is the maximum treatment mean value for the native range

$N_{\min }$ is the minimum treatment mean value for the native range

$S D_{I}$ is the pooled standard deviation for the introduced range

$S D_{N}$ is the pooled standard deviation for the native range

The pooled standard deviation $\left(S D_{l, N}\right)$ is calculated independently for each range as the average standard deviation for each population, in the high and low treatments. The pooled standard deviation for each range would be:

$$
S D_{I, N}=\sqrt{\frac{\sum_{i=1}^{n} S D_{i}^{2}}{n}}
$$

Where $S D_{i}$ is the standard deviation for each group, and $n$ is the number of groups (a group would be each population-treatment combination).

If we imagine a scenario where the native and introduced populations both share identical means in the high (mean $=8$ ) and low (mean $=4$ ) treatments. By looking at the reaction norm slopes to measure plasticity (i.e., the difference in means), these two ranges would have identical plasticity. However, if they have different pooled standard deviations, an SD of 2 in 
the introduced range and an SD of 4 in the native range, then $P_{\text {diff } v 1}$ would calculate them to have different levels of plasticity. This can be illustrated from applying Equation 5 :

$$
P_{\text {diff_v1 }}=\frac{8-4}{2}-\frac{8-4}{4}=2-1=1
$$

This would give an effect size of one for $P_{\text {diff } \_v 1 \text {, indicating that the introduced population has }}$ greater phenotypic plasticity by one standard deviation. When the difference between high and low treatments (i.e., the slope) is actually the same in the native and introduced ranges.

\subsubsection{Calculation of $\boldsymbol{P}_{\text {diff_v2 }}$ from Hedges' $\boldsymbol{d}_{\text {combined_SD }}$}

An option to remedy this incorrect estimate of the difference in plasticity $\left(P_{\text {diff_v1 }}\right)$, would be to use the mean pooled standard deviations of both ranges ( $\left.S D_{\text {combined }}\right)$ to standardise the difference in means. In other words, standard deviation would first be pooled across treatments within each range, and then pulled across ranges.

$$
S D_{\text {combined }}=\sqrt{\frac{S D_{\text {pooled_I }}^{2}+S D_{\text {pooled_N }}^{2}}{2}}
$$

Where: $S D_{\text {pooled_I }}$ is the pooled standard deviation of the introduced range.

$S D_{\text {pooled_N }}$ is the pooled standard deviation of the native range.

If we apply Equation 6 to the above scenario:

$$
S D_{\text {combined }}=\sqrt{\frac{4^{2}+2^{2}}{2}}=3.16
$$


Then use this combined standard deviation ( $\left.S D_{\text {combined }}\right)$ to standardise treatment responses in both ranges when calculating $P_{\text {diff_v2: }}$

$$
\begin{gathered}
P_{\text {diff_v2 }}=P_{i}-P_{n}=\frac{I_{\max }-I_{\min }}{S D_{\text {combined }}}-\frac{N_{\max }-N_{\min }}{S D_{\text {combined }}} \\
P_{\text {diff_v2 }}=\frac{8-4}{3.16}-\frac{8-4}{3.16}=1.27-1.27=0
\end{gathered}
$$

This gives a $P_{\text {diff_ } v 2}$ of 0 , which accurately reflects the lack of a difference in plasticity between ranges. Thus, I recommend using this combined standard deviation between the two ranges to standardise the calculation of plasticity in both ranges, so that they are in the same units of combined standard deviation. A hypothetical scenario of the different results that $P_{\text {diff_v1 }}$ and $P_{\text {diff_v2 }}$ give when calculating a difference in plasticity between two populations that have varying standard deviations can be seen Figure 21 . In this scenario $P_{\text {diff } v 1}$ is highly variable as the difference in standard deviation between populations changes, whereas $P_{\text {diff }} v 2$ stays relatively constant. 

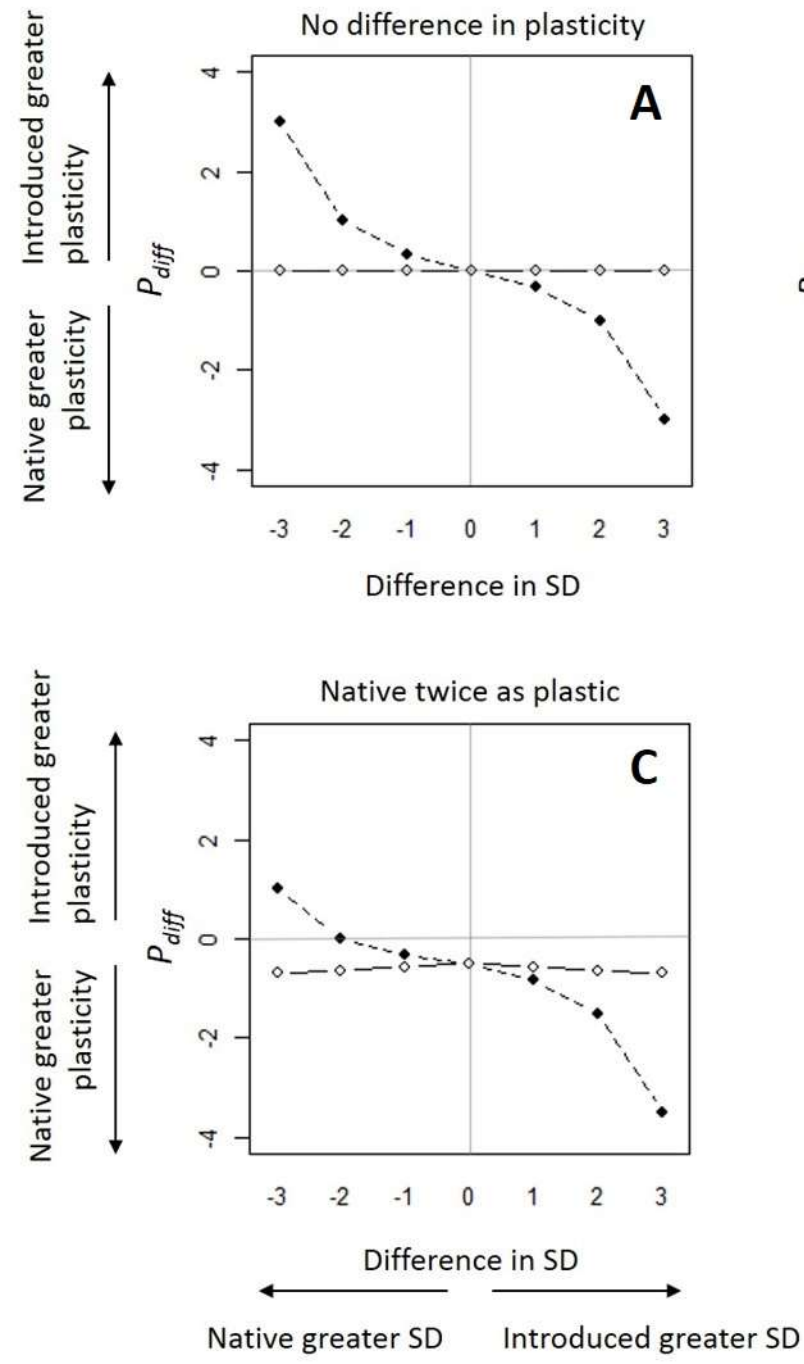

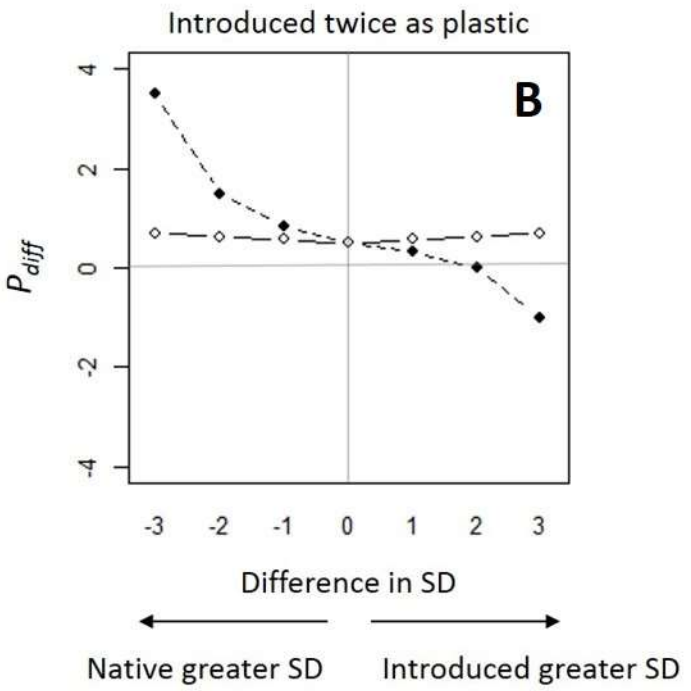

$$
\begin{aligned}
& \text { Legend } \\
& -P_{\text {diff_v1 }} \\
& \rightarrow P_{\text {diff_v2 }}
\end{aligned}
$$

Figure 21. Hypothetical scenarios comparing two effect size calculations of the difference in plasticity $\left(P_{\text {diff } \_v 1}, P_{\text {diff_v2 }}\right)$ between a native and introduced population. Differences in standard deviations between populations are shown on the x-axis. Difference in SD refers to the difference in standard deviation (SD) between populations; positive values indicate the introduced population have higher SD, negative values indicate the native population have higher SD. $P_{\text {diff }}$ describes the difference in plasticity between ranges; positive values indicate the introduced population have greater plasticity, negative values indicate the native population have greater plasticity. Panel $\mathbf{A}$ shows a scenario where there is no difference in 'true' plasticity between populations (i.e., they have parallel reaction norms). Panel B shows a scenario where the introduced population has twice the plasticity of the native populations (i.e., the slope of the reaction norm is twice as steep). Panel $\mathbf{C}$ shows a scenario where the native population has twice the plasticity of the introduced population. 


\subsubsection{Note on calculating Hedges' $d$}

When calculating Hedges' $d$ for a given trait, I used the regression slope (beta-coefficient) for the effect of treatment for each range from a linear mixed effects model (LME) with a random term for the Australian populations, on data that had been standardised by dividing each trait

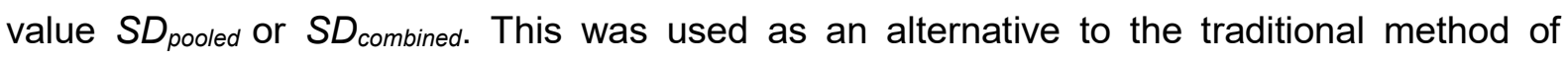
calculating Hedges' $d\left(d=\frac{\left(m_{1}-m_{2}\right)}{S D_{\text {pooled }}}\right)$. The approach of using the beta-coefficient from a LME gives similar values for the effect size and its variance, however it has the advantage of analysing the difference in mean values while taking into account random effects for multiple populations in the introduced and/or native range. This effectively gives a standardised betacoefficient, in which the data has been standardised by the pooled standard deviation between ranges $\left(S D_{\text {combined }}\right)$. It is possible to use standardised beta-coefficients as an effect size in a meta-analysis, when data is available to do so, as each effect size is now in units of standard deviation that are comparable between traits (Nieminen et al. 2013). Thus when betacoefficients are available, they can be used as a more accurate effect size.

\subsection{A case study comparison of the two approaches}

In the previous chapter (Chapter 5), I used this updated meta-analysis approach to calculate an overall effect size for the difference in plasticity between the native South African and introduced Australian populations. I have demonstrated mathematically how calculating a difference in plasticity from $P_{\text {diff } v 1}$, as in Davidson et al. (2011), causes a bias towards indicating an effect size with greater plasticity in the group that has a lower standard deviation. I now use real data to show how $P_{\text {diff_v2 }}$ gives a more reliable effect size than $P_{\text {diff_v } v}$, which reflect the actual differences in plasticity between two groups, using the data from Chapter 5 as an example.

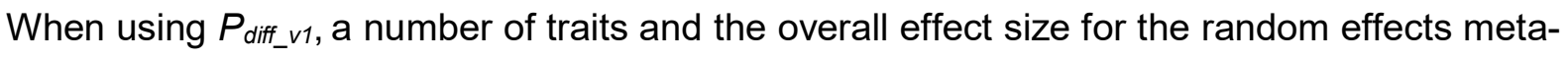
analysis model are biased towards indicating greater plasticity in the Australian populations 
(Figure 22; indicated by a positive effect size for $P_{\text {diff } v 1}$ ). This is due to a difference in standard deviations between ranges, where standard deviation tends to be greater in the native population (Table 11). When using $P_{\text {diff } v \text {, }}$, the effect size for each trait more accurately reflects the actual difference in plasticity between ranges. The differences in the effect sizes calculated

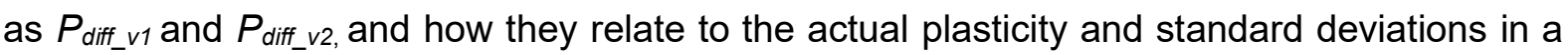
subset of example traits, are given in Table 11.

The effect size for four of the traits tested changed direction (nutrient treatment: LMF, RMF; water treatment: LMF, SSD), from a positive value when using $P_{\text {diff } v 1 \text { (introduced populations }}$ more plastic) to a negative value of $P_{\text {diff } v 2}$ (native population more plastic). As an example, leaf mass fraction (LMF) in the nutrient experiment had greater plasticity in the native population $(\beta=0.067)$ than in the introduced populations $(\beta=0.047)$. However, when $P_{\text {diff }} v 1$ was used this result was completely reversed, indicating that the introduced populations had greater plasticity $\left(P_{\text {diff } v 1}=0.66[95 \% \mathrm{Cl}:-0.2,1.53]\right)$. When using $P_{\text {diff } v 2 \text {, the effect size more }}$ accurately represented the lower plasticity in the native population $\left(P_{\text {diff } v 22}=-0.44[95 \% \mathrm{Cl}\right.$ : $1.86,0.98])$. The greater pooled standard deviation of the native population $\left(S D_{N}=0.08\right)$, compared to introduced populations $\left(S D_{l}=0.032\right)$, causes this discrepancy and bias when using $P_{\text {diff }}$ v1. 

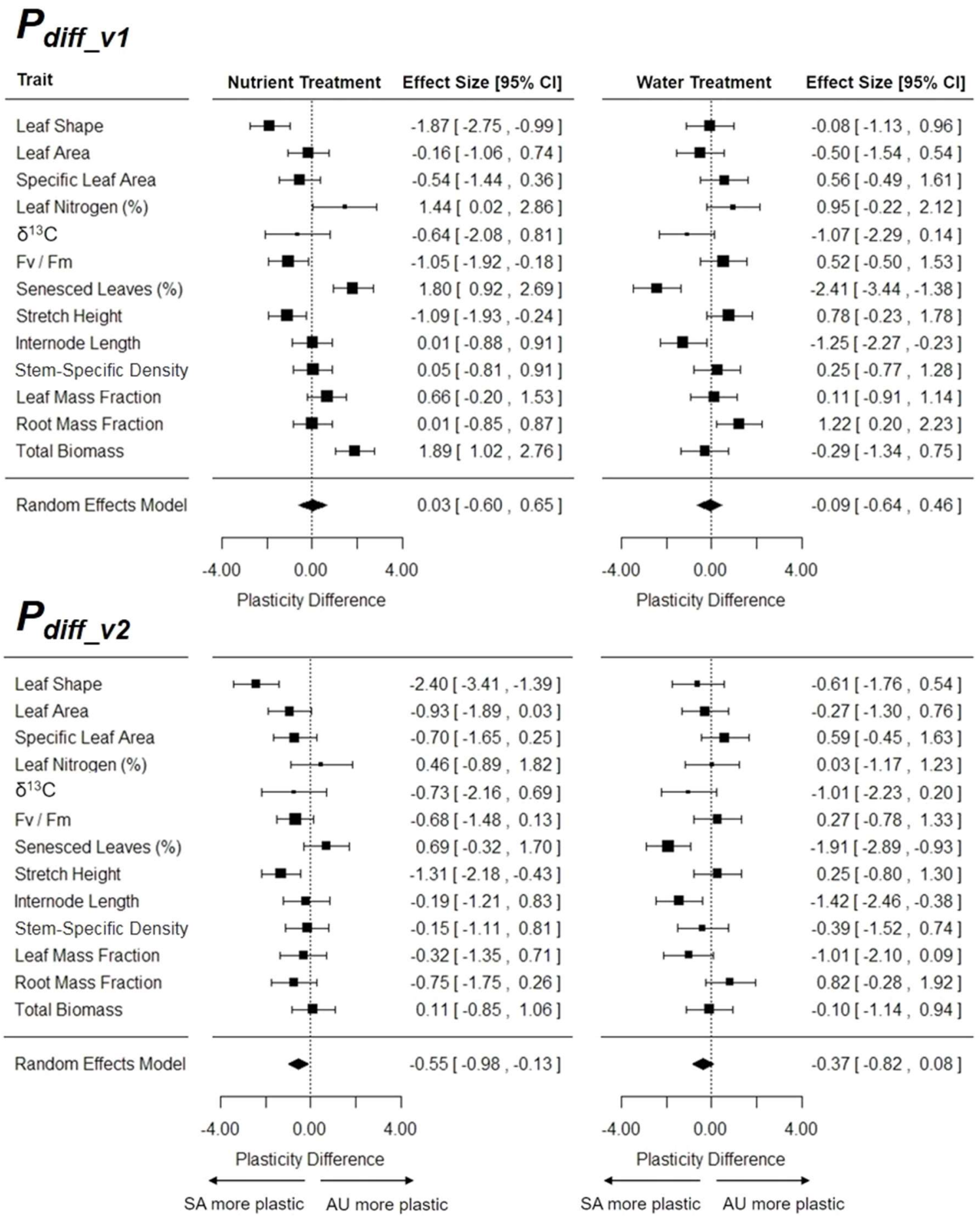

Figure 22. Meta-analysis for the difference in plasticity between native (SA) and introduced (AU) populations of Arctotheca populifolia to nutrient and water availability treatments, when the effect size $\boldsymbol{P}_{\text {diff }}$ is calculated as: $\boldsymbol{P}_{\text {diff_v1 }}$ (top) and $\boldsymbol{P}_{\text {diff_v2 }}$ (bottom). Effect sizes for $\boldsymbol{P}_{\text {diff }}$ values $>0$ indicate that the introduced populations had greater plasticity, $P_{\text {diff }}<0$ indicate that the native population had greater plasticity. The diamond symbol represents the overall effect size and confidence intervals, this was determined from a random effects meta-analysis model, which account for variance both within and between study variance. 
Table 11. Arctotheca populifolia traits that show highly different effect sizes for difference in plasticity between ranges $\left(P_{\text {diff }}\right)$, when calculated as $P_{\text {diff } v 1}$ or $P_{\text {diff } v 2 \text {. }} B$ shows the slope of the reaction norm between high and low treatments (i.e., the actual plasticity for that range). $S D_{\text {pooled }}$ is the pooled standard deviation of the high and low treatments in each range.

$P_{\text {diff }}$ values $>0$ indicate that the introduced populations had greater plasticity, $P_{\text {diff }}$ values $<0$ indicate that the native population had greater plasticity.

Acronyms: LMF, leaf mass fraction; RMF; root mass fraction; SSD, stem-specific density.

\begin{tabular}{|c|c|c|c|c|c|c|c|c|c|}
\hline \multirow{2}{*}{ Experiment } & \multirow{2}{*}{ Trait } & \multicolumn{2}{|c|}{ South Africa } & \multicolumn{2}{|c|}{ Australia } & \multicolumn{2}{|c|}{$P_{\text {diff_v1 }}$} & \multicolumn{2}{|c|}{$P_{\text {diff } v 2}$} \\
\hline & & $B$ & $\mathrm{SD}_{\text {pooled }}$ & $B$ & $S D_{\text {pooled }}$ & Effect Size & $95 \% \mathrm{Cl}$ & Effect Size & $95 \% \mathrm{Cl}$ \\
\hline Nutrient & LMF & -0.067 & 0.080 & -0.047 & 0.032 & 0.66 & $-0.2,1.53$ & -0.44 & $-1.86,0.98$ \\
\hline Nutrient & RMF & 0.042 & 0.036 & 0.021 & 0.018 & 0.01 & $-0.85,0.87$ & -0.96 & $-2.25,0.03$ \\
\hline Nutrient & $\begin{array}{l}\text { Total } \\
\text { Biomass }\end{array}$ & -0.861 & 0.217 & -0.881 & 0.15 & 1.89 & $1.02,2.76$ & 0.11 & $-0.85,1.06$ \\
\hline Water & LMF & 0.136 & 0.057 & 0.088 & 0.035 & 0.11 & $-0.91,1.14$ & -1.01 & $-2.1,0.09$ \\
\hline Water & Leaf $N$ & 1.413 & 0.815 & 1.395 & 0.531 & 0.95 & $-0.22,2.12$ & 0.03 & $-1.17,1.23$ \\
\hline Water & SSD & -0.015 & 0.020 & -0.009 & 0.009 & 0.25 & $-0.77,1.25$ & -0.39 & $-1.52,0.74$ \\
\hline
\end{tabular}




\subsection{Comment on alternative effect size index to calculate plasticity}

The response ratio $(R R)$ is another index that has been used in meta-analyses to look at differences in plasticity between invasive and native/non-invasive species (Palacio-López \& Gianoli 2011). It is calculated as the log proportional change between two treatment groups $\left(R R=\ln \left(x_{1} / x_{2}\right)\right)$. However, this effect size is limited, in that it will give an erroneous calculation for the variance for a group, if that group has a mean value very close to 0 . Consequently, it can also be inappropriate when comparing a difference in plasticity between two groups, as a group with very low plasticity (i.e., a slope value close to 0 ) will have an incorrect estimate for its variance (Lajeunesse 2015).

Palacio-López \& Gianoli (2011) used the response ratio as an effect size to compare levels of plasticty between native and invasive plant populations. However, rather than creating one effect size of the difference in plasticity (i.e., an effect size of an effect size, as outlined above), they calculated the plasticity seperately for two groups (native and invasive) as a response ratio, and then detemined if there was a signficant difference in plasticity from the overlap of a 95\% bootstrap confidence interval. Although this did not give them an overall effect size for the difference in plasticity, it avoids the issue that can arise when of calculating variance for a response ratio where one group has an effect size value close to 0 (i.e. a flat reaction norm indicating zero or very little plasticity). In their results they found no significant difference in plasticity between native and invasive groups (Palacio-López \& Gianoli 2011).

The use of the log response ratio can be a useful effect size, giving unique insight into proportional differences in size related traits (Borenstein et al. 2009b). However, if the trait is calculated as an index (e.g., such as many physiological traits), then the effect size will not meaningfully represent reaction norm slopes (i.e., the plasticity) that is comparable with other traits. When testing the response ratio on the data in the previous chapter, the physiological traits (e.g. $\left.F_{v} / F_{m}, \delta^{13} C\right)$, had very small effect sizes, that did not reflect the strength of their 
reaction norms. Thus it was deemed an inappropriate effect size to use for the purpose of this study.

\section{$\underline{6.5 \text { Discussion }}$}

Use of Hedges' $d_{\text {discrete_sD }}$ to calculate $P_{\text {diff } v 1}$, creates a bias towards indicating greater plasticity in the group that has lower intrinsic variation in trait values (i.e., lower standard deviation). This not only changes the magnitude of the effect size, but can also reverse its direction, relative to the true difference in reaction norm slopes. When applying this approach to calculate a difference in plasticity between ranges in Chapter $5, P_{\text {diff } v 1}$ indicated greater plasticity in the introduced populations than was intuitively revealed from the slopes of the reaction norms. When using Hedges' $d_{\text {combined_sD }}$ to calculate the effect size for $P_{\text {diff } v 2 \text {, the }}$ difference in plasticity more appropriately reflected the true difference in slopes between ranges.

The bias to give a higher $P_{\text {diff } v 1}$ value (i.e., overestimating plasticity in the Australian populations), was because the Australian populations tended to have less within treatment variation in trait values (i.e., lower standard deviation). This lower phenotypic variation in the introduced populations of $A$. populifolia might be unsurprising given the reduced genetic diversity present in these populations (Rollins et al. 2013). If introduced species do tend to have lower intra-population trait variation, then using a difference in Hedges' $d_{\text {discrete_sD }}$ to calculate a difference in plasticity will be biased to give an overall effect size of greater plasticity to invasive/introduced populations.

The original study conducted by Davidson et al. (2011) is a key part of invasive species literature. It has been cited 411 times to date, often used as evidence that invasive species have greater phenotypic plasticity than native non-invasive species. The implications for this potential bias in the calculation of the difference in phenotypic plasticity between native and invasive species is likely to have had a strong influence on the results in their study. An alternative explanation for their findings could be that invasive populations have lower intrinsic 
variation their native counterparts, if genetic variation is lost on introduction to the new range (Simberloff et al. 2000; Dlugosch \& Parker 2008). This highlights the importance of taking precautions when using more complex effect size indices to check that they accurately reflects the data they are based on. A re-examination of the data and results gathered in Davidson et al. (2011) is recommended for further investigation, with a comparison to the proposed method to calculate Hedges' $d$ outlined in this chapter. Unfortunately, this was not possible to be carried out with the time constraints permitted by this $\mathrm{PhD}$, but is planned to be explored further in the future. 


\title{
7. Do rates of trait change through time, trait differences between ranges, and climate niche shifts follow similar patterns? A case study of Arctotheca populifolia
}

\begin{abstract}
Predictions generated from species distribution models or herbarium specimens can be useful tools to assess how a species may alter its realised niche and/or traits when introduced to a new range. However, it is important to evaluate these prediction on real biological data. Here, I combine multiple lines of evidence, gathered from the previous three data chapters, to evaluate the different approaches used and to assess how Arctotheca populifolia may have changed since its introduction to Australia. Arctotheca populifolia showed distinct differences in morphological traits between native and introduced populations in a common garden glasshouse experiment. These differences were not reflected in trait change through time inferred from herbarium specimens. Additionally, present day trait differences between ranges in the glasshouse experiment better reflected the niche shift into a wetter climate, than the trait change through time inferred from herbarium specimens. This suggests that trait differences determined in glasshouse or common garden experiments, may be a more suitable approach to assess trait change in relation to a realised niche shift than using herbarium specimens. However, it is also possible that founder effects and other abiotic factors (e.g., burial, soil nutrients) that were not included in the assessment of realised niche shift, could also have a large influence on trait divergence between ranges.
\end{abstract}




\section{$\underline{7.1 \text { Introduction }}$}

In the previous three data chapters (Chapter $2-5)$, I have documented evidence for trait change through time in a number of introduced species (Chapter 3 ), related trait change through time to climatic niche shifts in the introduced species (Chapter 4), and demonstrated a loss of phenotypic plasticity in the introduced populations of Arctotheca populifolia (Chapter 5). While the use of herbarium specimens and species distribution models can be useful tools and provide a means to ask novel questions, it is important to validate answers from these methods against data from plant specimens grown in controlled conditions (Buswell et al. 2013; Hulme and Barret 2013).

If trait change through time measured from herbarium specimens accurately reflects adaptive change in A. populifolia since its introduction, then the direction of trait change should match current day differences in trait values between the native source and introduced populations. Herbarium specimens are becoming increasingly recognized a tool to track and examine change in trait values through time from historical specimens (Mcgraw 2001; Buswell et al. 2011; Flores-moreno et al. 2014; Dalyrmple et al. 2015). However, few of these studies also compare the slopes of trait change through time to trait differences between real plant populations. Law \& Salick (2005) found a decline in height through time in the intensely harvested Himalayan snow lotus (Saussurea laniceps). This coincided with lower mean height values in sites that were subject to greater harvesting versus sites where it was protected.

In this chapter, I will collate the results from Chapter's $3-5$, to give a clearer picture of trait divergence and trait-environment relationships in the introduced beach daisy, Arctotheca populifolia. When a species is introduced to a new range, many different processes may influence how that species responds to the new environment. Thus drawing from multiple distinct pieces of evidence will provide both a better overall picture of the processes that are shaping trait responses in A. populifolia. A comparison of the results will also give an idea of the reliability and consistency of the different methods used to assess them. The aim of this 
chapter is to combine the results of Chapters $3-5$, to examine trait divergence in relation to a realised niche shift in the introduced range of Arctotheca populifolia. To do this I address three key questions,

1. Does the direction of trait change through time in herbarium specimens correspond to the direction of the climatic niche shift?

2. Do differences in trait values between ranges correspond to the trait change through time in the herbarium specimens?

3. Do differences in trait values between ranges correspond to the direction of the climatic niche shift? 


\section{$\underline{7.2 \text { Methods }}$}

The methods in this chapter are the same as those used in the previous three chapters to collect data on and quantify: i) herbarium trait change through time, ii) climatic niche shifts, and iii) trait differences between the native source population and the introduced populations when grown in glasshouse conditions. These three aspects were then compared qualitatively to examine the consistency of patterns in trait-trait and trait-environment relationships between studies. There were some small adjustments in methodology that I will now outline.

\subsubsection{Niche shift in Arctotheca populifolia}

The magnitude and direction of climate niche shift in A. populifolia was assessed using the same approach as in Chapter 4.

\subsubsection{Does the direction of trait change through time in herbarium specimens correspond to the direction of climatic niche shift?}

To address question i), I quantified the slopes herbarium trait values against time, calculated from a spatial regression using general least squares analysis (GLS), with climate variables included as covariates. I used the same modelling approached as in chapter 3 to assess the rate and direction of trait change through time in $A$. populifolia, for plant height $(n=36)$, leaf area $(n=52)$, and leaf shape $(n=52)$. Various model combinations were tested to predict trait variation, which included terms for year, climate (temperature and moisture) and year $x$ climate interactions. All model combinations tested included a term for year. The best fitting model was then selected using Akaike's information criterion (AIC; Akaike 1973). The magnitude and direction of these slopes (change through time in each trait) were then compared to the realised niche shift which $A$. populifolia had undergone on introduction to Australia. As I was looking at a single species, I was able to pay particular attention to the direction of niche expansion along each climate axis, and the distribution of herbarium records in climate space. 


\subsubsection{Do differences in trait values between ranges correspond to the trait change through time in the herbarium specimens?}

To address question ii), plant traits were measured from plants grown in a common garden experiment, with seeds form the native source population in South Africa, and from four populations in the introduced range of the south-eastern seaboard of Australia (as in chapter 5). Leaf shape was re-assessed on the plants grown in glasshouse conditions, to keep consistency with the methods used to measure leaf shape on the herbarium specimens. Leaf shape was re-calculated as the ratio of leaf width to leaf length (as in Chapter 3; Buswell et al. 2011). Leaf shape assessed this way will hereafter be referred to as leaf shape ratio. Leaf shape calculated by dividing the perimeter by the area, as in chapter 5 , will hereafter be referred to as leaf shape perimeter.

\subsubsection{Do differences in trait values between ranges correspond to the direction of the climatic niche shift?}

To address question iii), I only considered plant specimens that had been grown in the water experiment. The niche shift analysis in Chapter 3 shows that $A$. populifolia primarily underwent a niche shift into a wetter climate (Figure 23). Thus comparing trait differences between ranges, in varying levels of water availability was most relevant to address this question. For this question I was also interested in looking at any trait differences between ranges, not just a difference in phenotypic plasticity. As in Chapter 5, all traits were first assessed using a linear mixed effect model, with main effect terms for treatment, range, the interaction of treatment $\times$ range, and a random effect for Australian population. The interaction term was removed from the model if it was not significant. The analysis was then repeated with only main effect terms for treatment and range in the model. If the interaction term was significant then the effect of range was tested independently in the high treatment and in the low treatment. Statistical analyses were carried out using the nlme package in R v.3.0.1 (R Core Team 2013). 


\section{$\underline{7.3 \text { Results }}$}

\subsubsection{Niche shift in Arctotheca populifolia}

Arctotheca populifolia underwent a realised niche shift into wetter climate on its introduction to Eastern Australia (Figure 23). This occurred through an expansion into non-analog climates (niche expansion into non-analog climate: $0.249-0.278$ [for niches including $75^{\text {th }}-99^{\text {th }}$ percentile of marginal climate space]). When only analog climates were considered there was no niche expansion, irrespective of the percentile of marginal climate space considered (niche expansion into analog climate: 0).There was a low amount of overlap between the two ranges (Schoener's $D=0.14$ ). Almost all of the herbarium specimens occurred in novel climate space (i.e., occurred outside the native range niche). The moisture index of the populations used in the common garden experiment was considerably higher in the introduced populations $(M=$ $0.9 ; L=0.79)$ than the native population $(A=0.36)$. Given this shift in niche space to an increase in moisture index in the introduced Australian range, trait change through time in the herbarium specimens and trait differences between ranges in the common garden experiment may be expected to reflect this.

\subsubsection{Does trait change through time correspond to the climate niche shift?}

Trait variation in response to year and climate was determined through selecting the best fitting model, as in Chapter 3. The best fitting model for plant height included a just a single term for year, and showed a slight increase through time, though this was not significant $(B=$ $0.0023, p=0.109)$. The best fitting model for leaf area included terms for year and moisture, but did not show any trend for change through time $(B=0.001, p=0.594)$. The best fitting model for leaf shape ratio included terms for year, temperature and the interaction of year $x$ temperature. Leaf shape ratio showed a significant interaction between year and temperature $(B=0.022, p=0.043)$, indicating that leaf shape was increasing through time in drier climates and decreasing through time in wetter climates (Figure 24). 


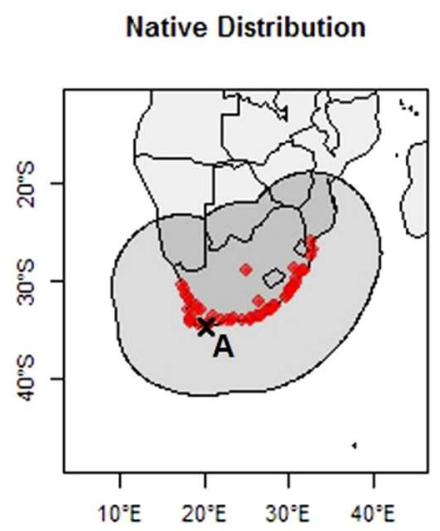

Introduced Distribution

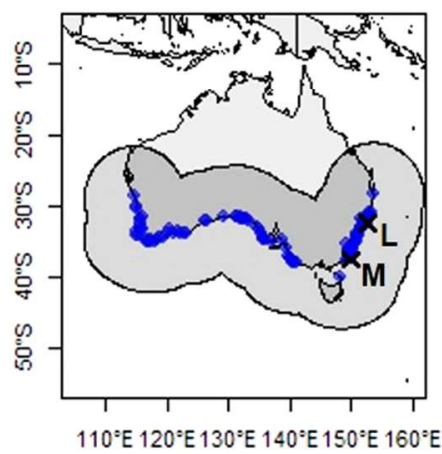

$110^{\circ} \mathrm{E} 120^{\circ} \mathrm{E} 130^{\circ} \mathrm{E} 140^{\circ} \mathrm{E} 150^{\circ} \mathrm{E}$ 160" E

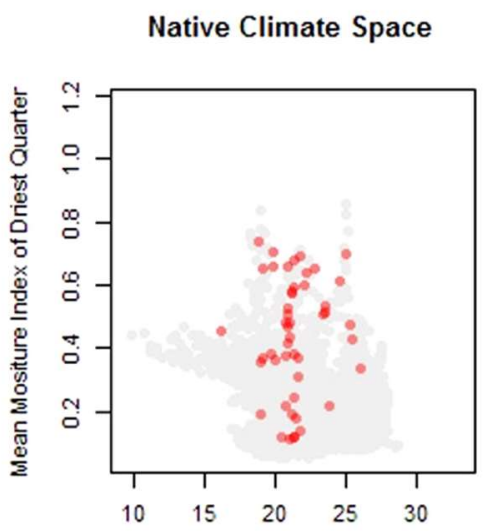

Mean Temperature of Warmest Quarter Introduced Climate Space

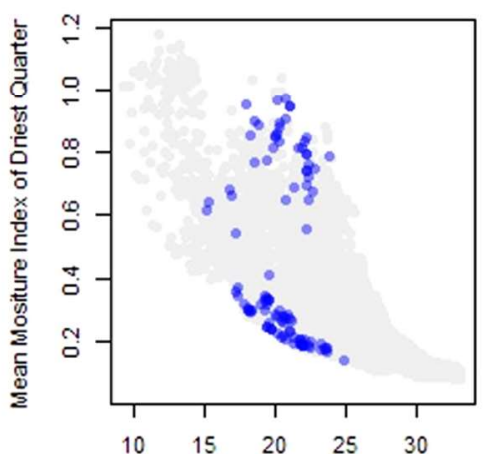

Mean Temperature of Warmest Quarter
Native Climate Densities

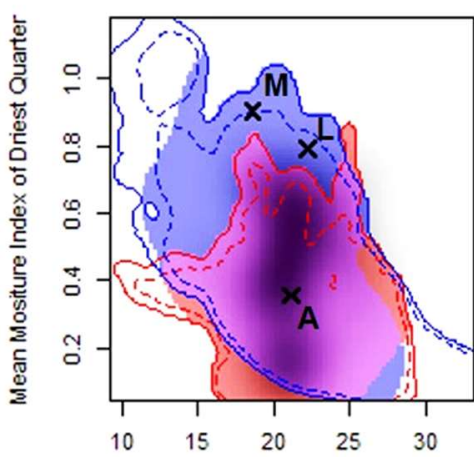

Mean Temperature of Warmest Quarter

Introduced Climate Densities

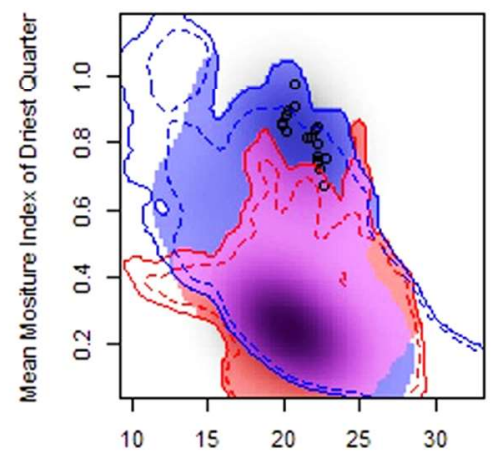

Mean Temperature of Warmest Quarter

Figure 23. The geographic distribution and realised climate niche of Arctototheca populifolia's native (top) and introduced (bottom) range. Left panels: Red (native) and blue (introduced) dots represent the locations for the native and introduced locations respectively, surrounding grey shading show the $800 \mathrm{~km}$ buffer in which background climate points were taken from. Middle panels: Red and blue dots represent the location of presences in climate space, grey dots represent the background points in climate space. Right panels: Solid lines represent the extent of the available climate space at the $99^{\text {th }}$ percentile for the native range (red) and introduced range (blue), dashed lines show this at the 75th percentile; coloured areas show the extent of climate space occupied in only the native range (red; niche unfilling), the introduced range (blue; niche expansion) and their overlap (purple; niche conserved); shading (greyscale) represents the densities (species occupancy) of the present locations in climate space for the native (top) and introduced (bottom) ranges; crosses (top panel) show the niche centroid for the native (red) and introduced ranges (blue); black circles (bottom panel) show the location in climate space of the herbarium specimens used to model trait change through time. 


\subsubsection{Do differences in trait values between ranges correspond to trait change through time in the herbarium specimens?}

The change in plant height through time measured from herbarium specimens was not significant $(B=0.002, p=0.109)$, although the direction of a slight increase does correspond with the Australian populations having greater stretch height across both treatments in the water experiment $(B=0.1, p<0.001)$, and in the low nutrient treatment $(B=0.173, p<0.001)$. Leaf area was significantly greater in the Australian populations across both treatments in the water experiment $(B=0.16, p=<0.001)$, and in the high treatment for the nutrient experiment $(B=0.179, p<0.001)$. However the herbarium specimens did not show a significant increase in leaf area through time $(B=0.0001, p=0.802)$. Herbarium specimens did show an increase in leaf area with increasing moisture $(B=0.114, p<0.001)$, which was consistent with the plastic response to high and low water treatment in both the native and introduced populations $(B=0.306, p<0.001 ;$ Figure 24).

There was no difference in leaf shape ratio between ranges when assessed in the nutrient experiment $(B=0.071, p=0.257)$. Leaf shape ratio was lower in the native populations in the water treatment experiment $(B=-0.071, p<0.001)$, this was mainly due to a greater decrease in leaf shape in the native plants in the low water treatment (Figure 24). Leaf shape ratio in the herbarium specimens showed a significant interaction between year and temperature $(B$ $=0.001, p=0.043)$, with leaf shape ratio decreasing through time in colder climates $(\mathrm{Q} 1=$ $66 \%$ ) and showing an increase through time in warmer climates (Q3 = 183\%; Figure 24). Moisture index was not found to have significant effect on leaf shape ratio as an independent term, or through an interaction with year $(p>0.05)$. 

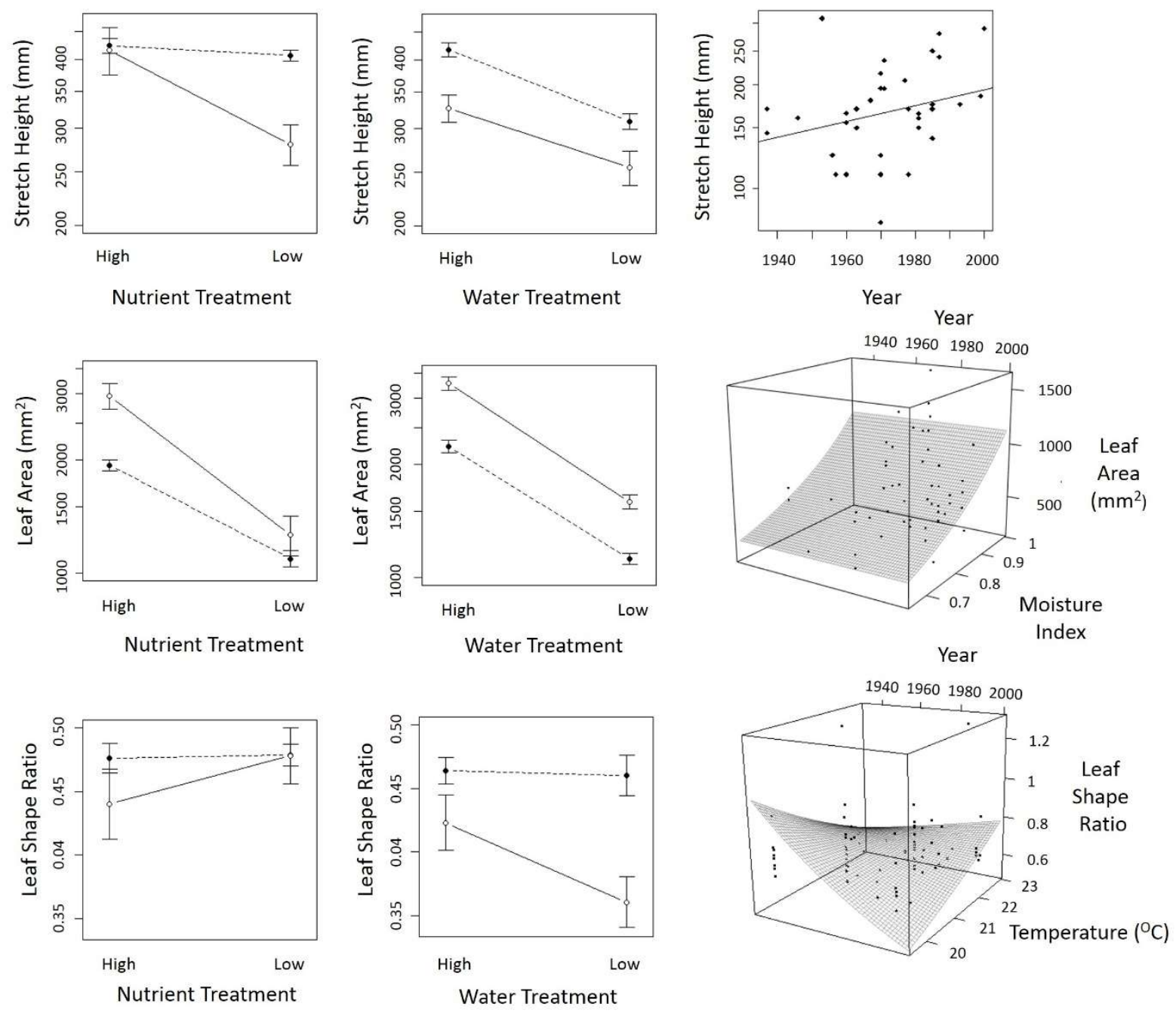

Figure 24. Trait differences expressed in a common garden nutrient treatment (left) and water treatment (middle) experiment; open circles represent the responses of the native population and solid circles represent the introduced range populations. Predicted trait change through time (right), measured from herbarium specimens with climate variables as optional covariates in the best fitting GLS model. 


\subsubsection{Do differences in trait values between ranges correspond to the direction of climate niche shift?}

Of the 13 traits assessed in the water treatment experiment, five showed a significant difference between ranges, with another three showing a significant effect for the range $x$ treatment interaction (Table 12; Chapter 5, Figure 19). The introduced populations showed a decrease in leaf shape $(B=-1.448, p<0.001)$, leaf area $(B=-0.16, p<0.001)$ and percentage leaf nitrogen content $(B=-1.405, p<0.004)$ compared to the native population, across both high and low water treatments. The introduced populations also showed greater stretch height $(B=0.1003, p=0.002)$ and chlorophyll fluorescence $\left(F_{v} / F_{m} ; B=0.007, p=0.002\right)$, across both treatments.

A significant treatment $\times$ range interaction occurred in three traits due, to a significant difference between ranges in either the high water treatment or low water treatment, but not both. Stem internode length was significantly lower in the introduced populations in the high water treatment $(B=-0.142, p=0.01)$. The Australian populations had a significantly lower percentage of senesced leaves $(B=-11.945, p<0.001)$ and leaf mass fraction $(B=-0.065, p$ $<0.001$ ) in the low water treatment (Table 12; Chapter 5, Figure 19). 
Table 12. Trait differences between the native source population and introduced populations of Arcotheca populifolia, in response to water availability. All traits were first fitted with a linear mixed effects model, including terms for the treatment, range, and their interaction (plus a random term for the Australian populations). If the treatment $\times$ range interaction was significant then the effect of range was tested in the high treatment and in the low treatment independently. If there was no significant effect for the interaction, then the interaction term was removed and the main effects of treatment and range were tested. Acronyms: SLA, specific leaf area; SSD, stem-specific density; RMF, root mass fraction, LMF, leaf mass fraction.

\begin{tabular}{|c|c|c|c|c|c|c|c|c|c|c|}
\hline \multirow[t]{2}{*}{ Trait } & \multicolumn{2}{|c|}{ Treatment } & \multicolumn{2}{|c|}{ Range } & \multicolumn{2}{|c|}{$\begin{array}{l}\text { Range } \times \\
\text { Treatment }\end{array}$} & \multicolumn{2}{|c|}{$\begin{array}{c}\text { Range } \\
\text { (High Treatment) }\end{array}$} & \multicolumn{2}{|c|}{$\begin{array}{c}\text { Range } \\
\text { (Low Treatment) }\end{array}$} \\
\hline & B & $p$ & B & $p$ & B & $p$ & B & $p$ & B & $p$ \\
\hline Leaf shape complexity & 0.169 & 0.323 & -1.448 & $\leq 0.001$ & & & & & & \\
\hline Leaf area* & -0.306 & $\leq 0.001$ & -0.16 & $\leq 0.001$ & & & & & & \\
\hline SLA (mm/mg) & -0.041 & 0.129 & -0.011 & 0.891 & & & & & & \\
\hline Leaf Nitrogen (\%) & 0.553 & $\leq 0.001$ & -1.405 & $\underline{0.004}$ & & & & & & \\
\hline$\delta^{13} \mathrm{C}$ & 2.231 & $\leq 0.001$ & -0.247 & 0.361 & & & & & & \\
\hline$F_{v} / F_{m}$ & -0.008 & $\leq 0.001$ & 0.007 & $\underline{0.002}$ & & & & & & \\
\hline Senesced leaves (\%) & 1.155 & 0.44 & -2.255 & 0.236 & 14.3 & $\leq 0.001$ & 2.255 & 0.503 & -11.945 & $\underline{0.001}$ \\
\hline Stretch height* & -0.125 & $\leq 0.001$ & 0.1003 & $\leq 0.001$ & & & & & & \\
\hline Internode Length* & -0.145 & $\underline{0.02}$ & 0.142 & $\underline{0.004}$ & -0.249 & $\underline{0.004}$ & -0.142 & $\underline{0.01}$ & 0.246 & 0.101 \\
\hline $\operatorname{SSD}\left(\mathrm{mg} \mathrm{mm}^{-1}\right)$ & -0.011 & $\leq 0.001$ & -0.002 & 0.627 & & & & & & \\
\hline RMF & -0.0098 & $\underline{0.002}$ & -0.0064 & 0.058 & & & & & & \\
\hline LMF & 0.088 & $\leq 0.001$ & -0.017 & 0.266 & 0.047 & $\underline{0.03}$ & -0.017 & 0.22 & -0.065 & $\leq 0.001$ \\
\hline Total Biomass* & -0.493 & $\leq 0.001$ & -0.032 & 0.471 & & & & & & \\
\hline
\end{tabular}

${ }^{*}$ Size related traits that have been $\log _{10}$ transformed 


\section{$\underline{7.4 \text { Discussion }}$}

\subsubsection{Does trait change through time correspond to the climate niche shift?}

I first asked if trait change through time, inferred from herbarium specimens in the introduced range of Arctotheca populifolia, corresponded with what would be expected in relation to the climate niche shift between the native and introduced range. This is essentially a repeat of the question I asked in Chapter 4 of this thesis, but with a specific focus on an individual species. Arctotheca populifolia underwent a niche shift into wetter climate in the introduced Australian range. This did not appear to drive significant trait change through time in herbarium specimens. Plant height showed a slight increase through time, which may be expected with a shift into wetter climate (Moles et al. 2009; Moles et al. 2011), however this was not significant. Leaf area showed no change through time in the herbarium specimens, but did show a highly plastic response to moisture index by increasing across a moisture gradient. Despite undergoing a niche shift into wetter climate, leaf shape ratio in herbarium specimens did not show a significant response to varying moisture availability at the landscape scale. Leaf shape ratio was predicted to change through time in different directions across different temperatures, however Arctotheca populifolia showed little shift along an axis of temperatureis on its introduction to Australia

Although Arctotheca populifolia showed a large degree of niche expansion on introduction to Australia, nearly all of this is due to expansion into non-analog climate space. As the native South African niche filled nearly all of the climate space available to it, there was no niche expansion when only analog climates were considered. Thus, it is difficult to determine if the niche expansion into wetter climate represents niche evolution, or simply the expansion into climate space that it was already pre-adapted to (Petitpierre et al. 2012; Guisan et al. 2014). Nevertheless, this shift into wetter climate could still expose $A$. populifolia to novel selection pressures in the introduced range that may drive adaptation to the new environment (Pearman et al. 2008; Alexander \& Edwards 2010). 


\subsubsection{Do differences in trait values between ranges correspond to trait change through time in the herbarium specimens?}

When grown in common garden glasshouse conditions, stretch height was greater in the Australian populations in the water treatment experiment and when nutrients were limiting in the nutrient treatment experiment, relative to the South African population. The direction the difference in height between ranges corresponded with the increase in height through time observed in the herbarium specimens, however this latter trend was not formally significant $(p$ $=0.109)$. Buswell et al. (2011), working with much the same data, detected a significant increase in height through time. However, this was not consistent with my analysis. This is because three herbarium specimens could not be geo-referenced and were removed from the dataset. Two of these were old samples, with low height values, and consequently had a relatively large influence in the analysis, such that their omission resulted in a non-significant effect of year. It is possible that a significant increase in height through time could be detected if there were more herbarium samples.

The introduced Australian populations showed distinctly lower leaf area than the native population, in both the water treatment and nutrient treatment experiments. This did not correspond with the herbarium analysis, which showed no change in leaf area through time However, leaf area did show high levels of phenotypic plasticity to water and nutrient availability in both the native and introduced populations, which was consistent with an increase in leaf area over a gradient of moisture availability in the herbarium specimens. This indicates that leaf area is a particularly plastic trait in both the native and introduced range. It is therefore possible that environmental variation at a local scale may have masked a trend of leaf area change through time being detected from herbarium specimens.

Leaf shape ratio was lower in the Australian populations in the water treatment experiment, but not in the nutrient treatment experiment. Herbarium specimens indicated that leaf shape ratio was changing at different rates in different temperatures. However, this does not point 
towards any clear pattern between leaf shape ratio change through time and present day differences in mean in leaf shape ratio between ranges.

Overall, the results indicate that trait change through time in herbarium specimens were not reflected in present-day differences in mean trait values between ranges. This is largely due to trait differences measured between populations in the common garden experiment not being reflected in the trait change through time observed in herbarium specimens. It could be that trait change was not detected by a log-linear prediction, as specified in the GLS models used. This could be for a number of reasons, including: i) phenotypic plasticity and local-scale environmental heterogeneity creating excessive variation in the herbarium samples, which would mask a linear trend from being detected, ii) the change in trait values may not have occurred as a linear response through time, but rather as a sudden change that could be due to founder effects (Mayr 1942; Williams et al. 2008) or a rapid selective sweep on introduction to the new range (Anderson et al. 2011; Stapley et al. 2015). A greater number of specimens from early on in the colonisation process might increase power to detect early, rapid changes in phenotype.

\subsubsection{Do differences in trait values between ranges correspond with a realised climate niche shift?}

The introduced Australian populations experienced a far greater moisture index of the driest quarter of the year $(0.79-0.9)$ compared to the native source population $(0.36)$. Consequently trait differences between ranges would be expected to reflect this increase in soil water availability.

Leaf carbon isotope (leaf $\delta^{13} \mathrm{C}$ ) composition can be used a measure of intrinsic water use efficiency (Siebt et al. 2008). Despite the increase in soil moisture available in the introduced populations, there was no significant difference in leaf $\delta^{13} \mathrm{C}$ composition between ranges. However, there was a strong effect of water treatment in both ranges, indicating that both ranges were able to exhibit sufficient plasticity to significantly increase their water use 
efficiency when water is limiting. This indicates that the introduced populations have not adapted to an increase in water availability in their new range, by directly reducing their intrinsic water-use efficiency. It is possible that sufficiently high levels of pre-adapted plasticity can shield genotypes from selection, and constrain the rate of adaptive evolution (Stearns 1982; Levin 1988; Ghalambor 2007). Alternatively, it is possible that other traits that influence water use efficiency may have adapted to the change in water availability instead.

Leaf senescence can contribute to plant survival under drought conditions (Munné-Bosch \& Alegre 2004). The Australian populations had considerably lower plasticity for leaf senescence. In contrast, the South African population has much greater plasticity, through a large increase in leaf senescence in the low water treatment. It is possible that the reduced plasticity in leaf senescence, could have been selected for in the Australian populations following a shift into wetter climate if it was costly to maintain (DeWitt \& Wilson 1998). Similarly, the increase in height observed in the Australian populations may have been an adaptive response to an increase in soil moisture in the introduced range (Moles et al. 2009; Moles et al. 2011; Ahmadikhah \& Maurufinia 2016).

The source South African population had greater leaf shape complexity, across both water treatments, as well as lower leaf shape ratio (width to length ratio) when water was limiting. These are both leaf characteristics that are associated with better temperature regulation and water use efficiency (Peppe et al. 2011; Guerin et al. 2012). Thus, the shift to more uniform, wider, and less complex leaf shapes in the Australian populations could be a selective process as water use-efficiency is a less pressing issue in the wetter Australian climate niche. Brandenburger et al. (2017) also observed lower leaf shape complexity in the Australian populations of $A$. populifolia, suggesting that this could be a case of neoteny: the retention of juvenile characteristics in adult individuals. The high plasticity in leaf shape complexity to nutrient availability in the South African population was manifested through a reversion to simple ovate leaves when nutrients were limiting. This could be because plants were unable to develop fully-formed, mature leaves when resources were severely limiting. 
Both the native and introduced populations showed high plasticity in leaf area, with parallel reaction norms for a decrease in leaf area when water was limiting in the common garden experiment. This decrease in leaf area follows what would be expected under an increase in water stress (Rucker et al. 1995; Khan et al. 2001; Xu et al. 2009). However, despite the direction of this plastic response to water availability, the introduced populations had lower leaf area. This suggests that it is unlikely that the decrease in leaf area was selected for because of a shift into wetter climate in the introduced range. Thus, it is plausible that other abiotic or biotic factors may have caused this decrease in leaf area in the introduce range.

Plant chlorophyll fluorescence $\left(F_{v} / F_{m}\right)$ was higher in the Australian populations, indicating greater plant health (Molina-Montenegro et al. 2013). This may be due to the Australian populations being generally fitter than the native source population, or because the glasshouse treatment conditions were more detrimental to the health of the native source populations. For example, both the high and low water treatments could have been suboptimal conditions for the native population (with the optimum water level ranging somewhere in the middle). However, there was no difference between ranges in the mean trait value or phenotypic plasticity for total plant biomass, a key fitness-related trait that integrates many proximate physiological and morphological responses (Voille et al. 2007). This suggests that despite undergoing a shift in the realised niche, and exhibiting present day differences in a number of traits, the overall impact on plant performance may have been neutral.

Measures of reproductive traits (i.e., seed output, reproductive biomass) would have been desirable in this study, in order to make more robust comparisons of ultimate plant fitness between ranges and treatments. Reproductive trait data was initially collected in this study, however consistent differences in the phenology of flowering time between ranges (the Australian populations flowered approximately two months earlier) meant that any comparison would confound reproduction with age and generation time. Thus, reproductive traits were opted to be excluded from the results. Current work is underway by other researchers, 
exploring reproductive differences between the native and introduced populations of $A$. populifolia.

There was a lack of a strong concordant signal between the climate envelope analysis, the common garden experiment and the herbarium specimens. This means that the realised niche shift $A$. populifolia underwent when introduced to Australia was likely through an expansion into climate space that it was already pre-adapted to. However, this shift into wetter climate could still expose populations to novel section pressures that could facilitate a change in some traits. Overall, differences in trait values between the native and introduced populations of $A$. populifolia were mixed in relation its shift in realised climate niche. This could be because certain traits may not have the same genetic capacity to rapidly adapt as others, or may experience differing degrees of selection pressure, in part because they may already be highly plastic. Alternative explanations for the observed differences in trait values between ranges include: i) other biotic or abiotic selection pressures in the introduced range may have had a stronger influence on driving trait change, iii) founder effects may have resulted in a distinct, but effectively random, set of trait values compared to the mean(s) expressed in the native range population, and iii) a combination of the above factors may have acted in opposition or in concert to influence the trait values and phenotypic plasticity now present in the introduced Australian populations.

\subsubsection{Global climate dataset versus real site conditions}

The niche shift analysis indicated a shift to higher soil moisture availability in the introduced range. However, this might not necessarily translate into an increase in plant water availability. In Australia, A. populifolia tends to grow on large free-draining sand dunes, while in South Africa it often grow over bedrocks in little pockets of sand, with higher organic matter content and more nutrients (Creer, personal communication, April, 2015). Thus, despite experiencing reduced moisture in South Africa (modelled at a coarse global scale; Kriticos et al. 2012), the shallow soils above a water-impermeable bedrock and greater water-holding capacity of 
higher organic matter soils may offset this, increasing the water available at the microsite of roots. This could also explain why some of the trait differences between ranges did not coincide with what would be expected from the assumption of an increase in water availability.

\subsubsection{Alternative abiotic factors: Burial}

It is possible that differences in other biotic or abiotic factors exerted a greater selective pressure than climatic differences between the native and introduced range. In Australia, $A$. populifolia tends to grow at coastal beach sites on mobile sand dunes, whereas in South Africa they tend to occur further from the shoreline, in little pockets of sand over bedrock with more nutrients and soil organic matter (Creer, personal communication, April, 2015). The closer proximity to the sea, greater volume of sand and higher wind velocities experienced in the Australian sites (Creer, personal communication, April, 2015), will likely increase the frequency and intensity of burial events experience by A. populifolia (Hesp 2002; Fisher 2008). A key adaptation of plants susceptible to burial is an increase in vigour or growth (Maun 1998; Samsone et al. 2009). This could explain why the Australian populations maintained greater stem length when nutrients were limiting, but did not show an increase in plant biomass, as might be expected if they had simply adapted to be better suited to the lower nutrient conditions in Australia.

Sand dune environments can subject plants to high levels of mechanical disturbance and environmental stress via burial, potentially resulting in high rates of mortality (Maun 1998; Gilbert et al. 2008). If an individual is not adapted to keep up with rates of burial through high stem growth rates, tolerance of darkness, or storing energy in their leaves, it will likely result in death of the plant (Wilson \& Sykes 1999; Maun et al. 1996; Maun 2009). Thus, it is possible that differences in the dune environment, and the cycling and rate of burial events could have resulted in a sudden selective sweep upon introduction to Australia. This could be why there are some dramatic trait differences between ranges that have occurred over a short space of time since $A$. populifolia's introduction to Eastern Australia (Approximately 80 years ago). This 
may also explain why some of the trait differences between ranges were in a counter direction to what would be expected following a niche shift into wetter climate. For example, leaf area is generally expected to increase with increasing water availability (Fonseca et al. 2000; Peppe et al. 2011), but showed a decrease in the Australian populations following a shift into wetter climate. An alternative explanation for this is that leaf area may decrease in response to burial, in a trade-off with increased photosynthetic rate per unit leaf area (Shi et al. 2004). Following this, I feel that other abiotic differences between ranges, such as burial and substrate properties, are more likely to have had a greater influence on trait change in $A$. populifolia than the detected niche shift into wetter climate.

The implications of burial are also important to consider in regards to the sampling of herbarium specimens. Herbarium specimens were much shorter than plants grown in glasshouse conditions. An inspection A. populifolia in the field in Australia revealed that their stems continued extensively underground (i.e., they were subject to considerable burial; Personal Observation). The herbarium records of $A$. populifolia look like they were sampled from the extent of the plant that occurred above ground (Personal Observation; Figures S43S47). Thus, the herbarium specimens of $A$. populifolia are unlikely to accurately represent the true height of that plant. Ironically, the potentially misleading evidence for $A$. populifolia to show an increase in plant height through time in Buswell et al. (2011), formed the basis for selecting this plant for further research that revealed trait differences between the native and introduced ranges in in morphology (Brandenburger et al. 2017), physiology (Brandenburger et al. In prep) and phenotypic plasticity (Chapter 6 of this thesis). 


\subsection{Summary}

Arctotheca populifolia showed distinct differences in a number of traits between the native and introduced range populations, across nutrient and water treatment experiments. These trait differences were apparent, but did not correspond with trait change through time inferred from herbarium specimens (apart from a non-significant increase in height through time). This indicates that measures of historic herbarium specimens may not be a very reliable way to infer trait change through time in introduced species, particularly as there are multiple factors that could result in a non-linear trait response. In the case of $A$. populifolia, this is revealed through the detection of distinct differences in mean trait values between ranges, but a lack of evidence for trait change through time (i.e., false negatives in plant height and leaf area). It is of course possible, though unlikely, that the Australian population has shown trait conservatism since the time of its introduction, and that the South African population has evolved away from its previous phenotype over the same period.

Measured, present-day trait differences between ranges did show a better match with the niche shift into a wetter climate than trait change through time in herbarium specimens. This suggests that mean trait differences between populations may be a better approach quantify trait change in relation to a change in environment in introduced species. However, some trait differences were likely to be caused by other factors that were not included in quantifying the species' niche shift (i.e., difference in sand dune dynamics). This highlights an issue with attempting to predict specific trait responses, from coarse scale measures of a species climatic niche shift. Certain traits may come under a multitude of simultaneous selection pressures when introduced to a new range, which may act in concert or opposition in selecting for a new optimum phenotype (Felker-Quinn et al. 2013). While climate variables are often used in determining a species' niche, as the data is fairly easy to obtain, they may miss key processes that are influencing a species' distribution and/or trait expression. This can add additional uncertainty when attempting to predict or interpret species trait responses from a shift in their realised climate niche. This also suggests caution should be used when climate change 
experienced through a transfer in space is used as a proxy for predicting species responses to in situ climate change over time. The space for time substitution may be on shaky ground because of the different ways that founder effects and/or adaptive evolution may respond to each specific situation. In addition to the potential for confounding differences in other nonclimatic abiotic and biotic factors.

\subsection{Appendix D - Supplementary material}
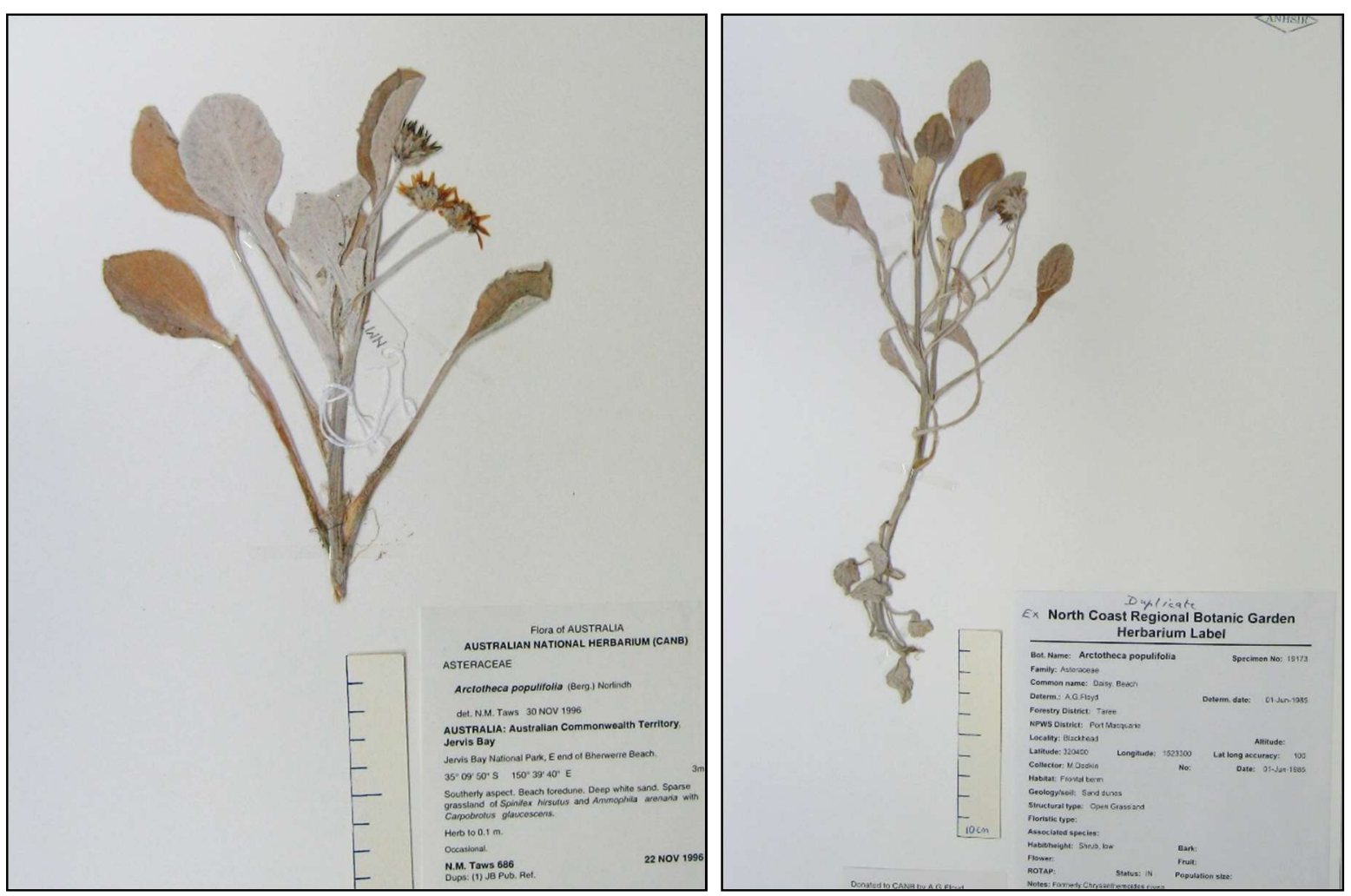

Figure S43. Herbarium specimens of Arctotheca populifolia. 

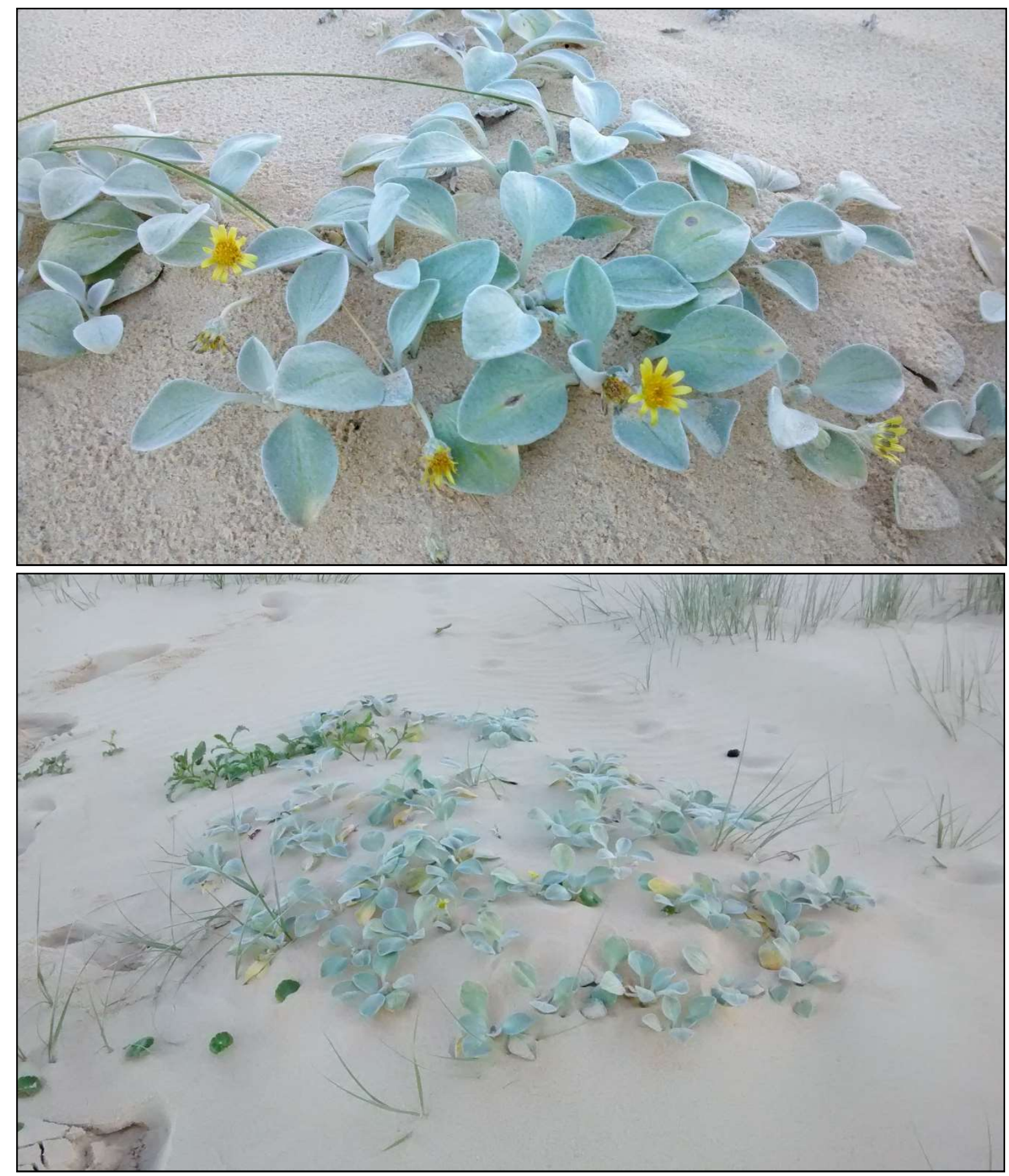

Figure S44. Arctotheca populifolia growing on sand dunes in Australia (Wairo beach). This is most likely to be one single plant. 


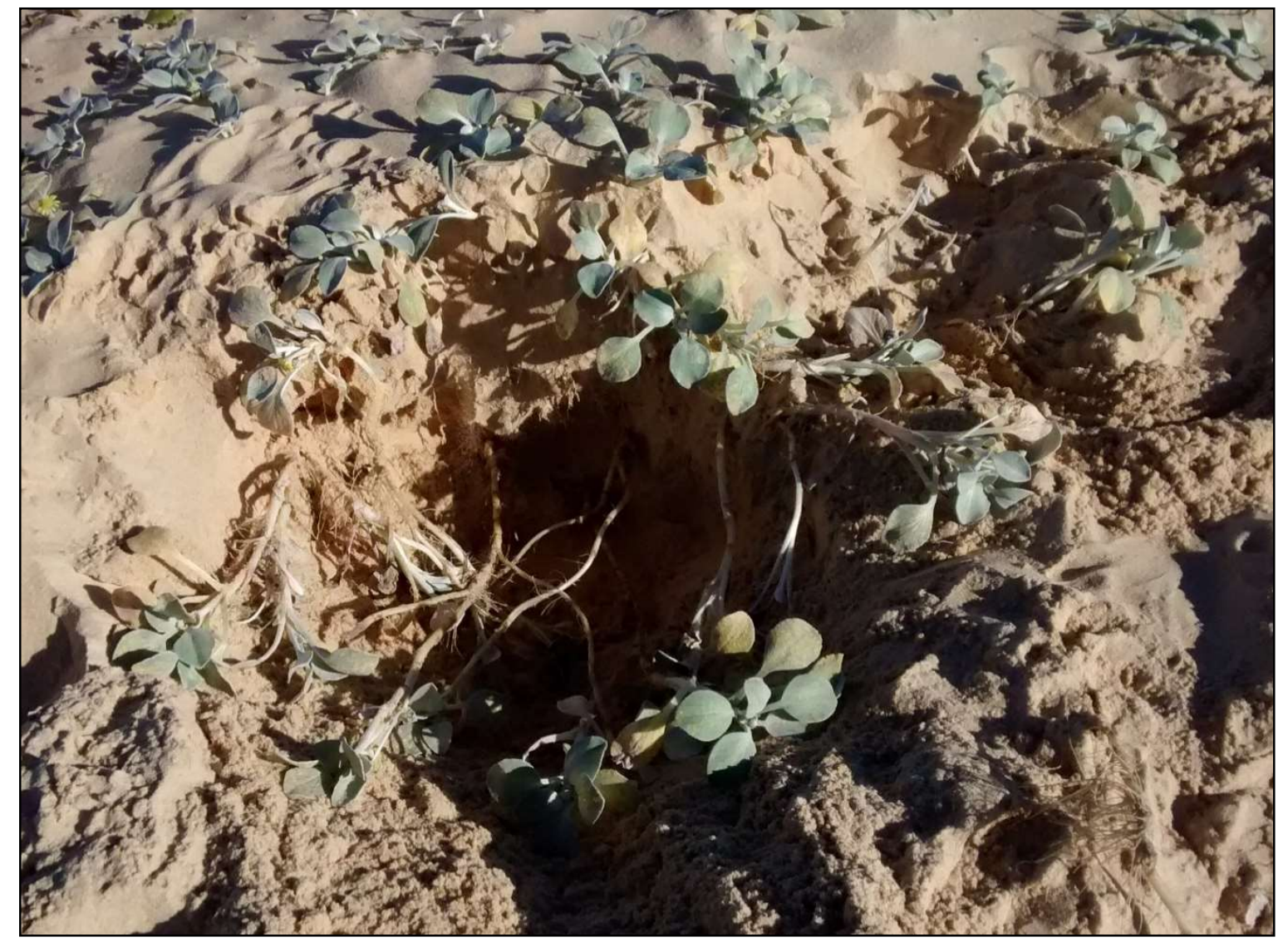

Figure S45. The complex deep rooting/branching system of Arctotheca populifolia, in Australia (Wairo beach). This appears to be a single plant, connected by a multitude of branches. 


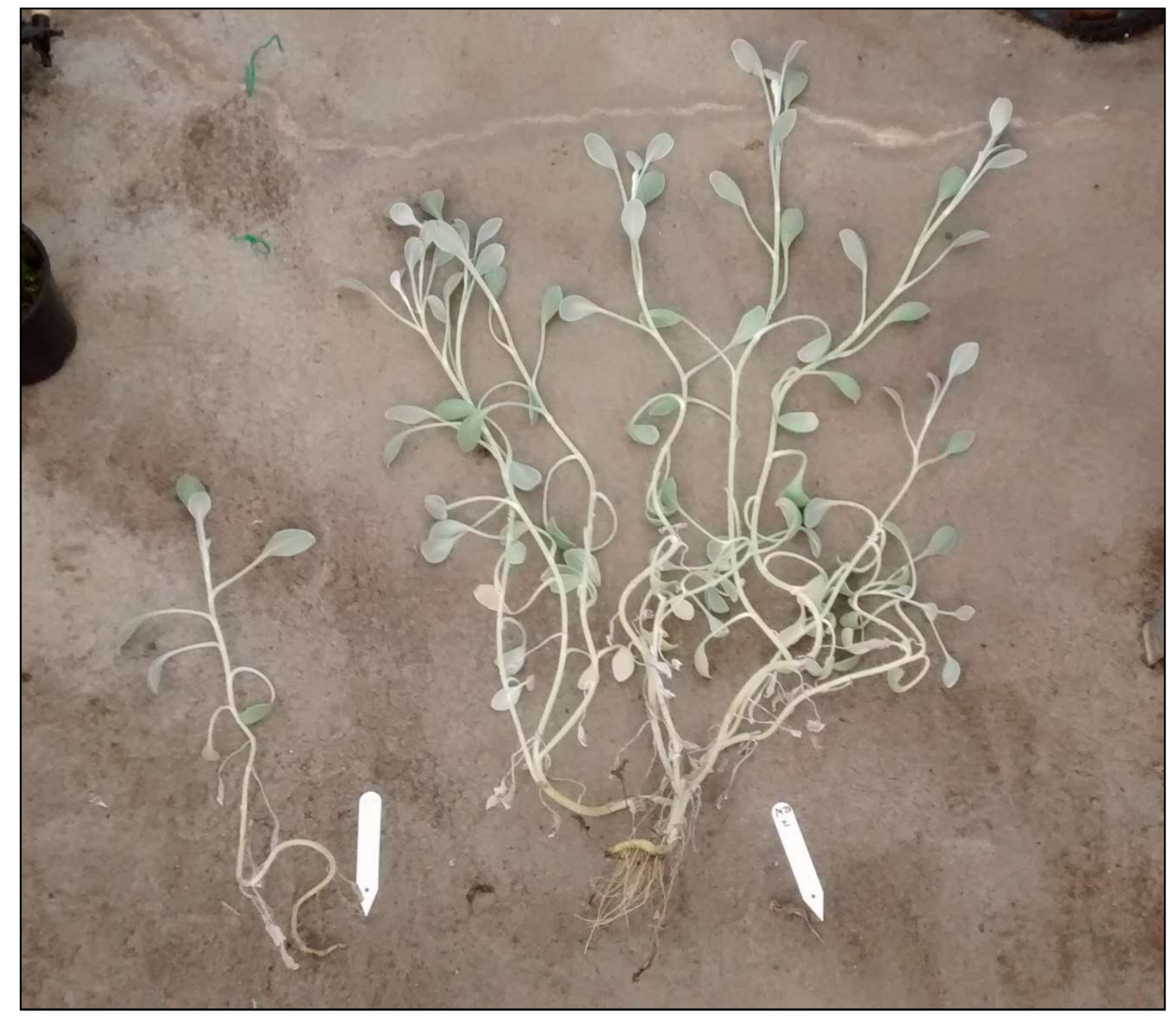

Figure S46. An introduced Australian maternal pair of Arctotheca populifolia at harvesting, grown in the low nutrient treatment (left) and in the high nutrient treatment (right). 


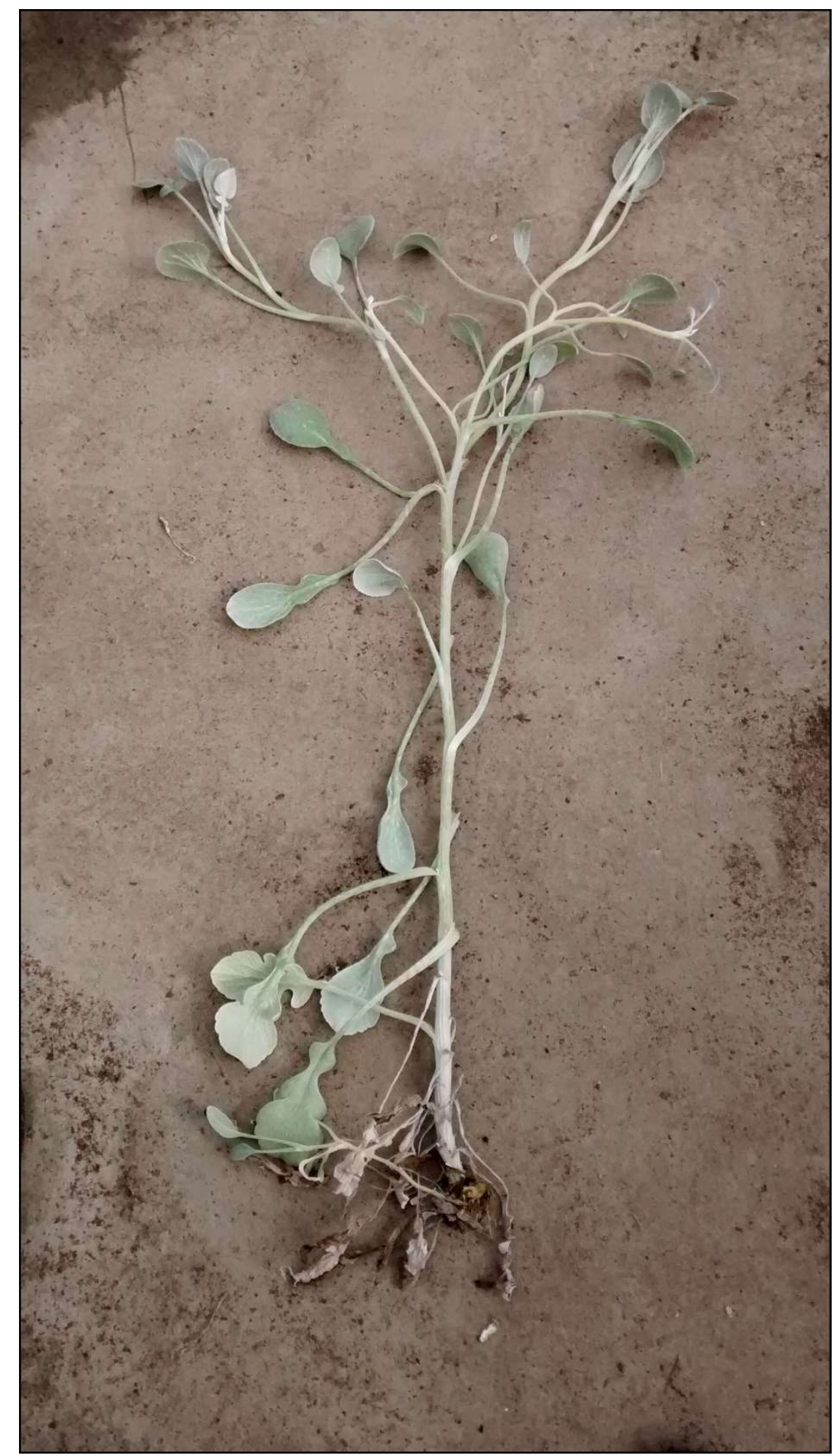

Figure S47. Arctotheca populifolia from the native South African population, grown in the high nutrient treatment. 


\section{Part IV \\ Discussion}




\section{General Discussion}

\subsection{Recap of thesis findings}

In this thesis, I assessed how traits in multiple non-native plant species may be changing when introduced to a new range, how this trait change may relate to climate niche shifts, and how phenotypic plasticity may also rapidly change in one species-specific example.

Previous research has indicated evidence for trait change through time in plant species introduced to Australia and New Zealand, based on morphological trait measures taken from historic herbarium specimens (Buswell 2009; Buswell et al. 2011). Re-analysis of these species, with the inclusion of climate and location data, made a number of differences to models predicting the rate of trait change through time. Most of these changes were through the detection of a significant year $\times$ climate interaction. The significant effect of a climate $\times$ year interaction indicates that trait change through time was occurring at different rates in different climates. This suggested that climate might be a driver of trait change through time in these introduced plants, which could be a result of climatic differences between the native and introduced ranges.

If climate was driving trait change through time, then the rate and direction of change may be expected to relate to differences in climate niche space occupied between the native and introduced ranges. However, a meta-analysis revealed that there was little evidence for: i) the magnitude of climate niche shift predicting trait change through time, and ii) trait change through time occurring at a faster rate in novel climate space. These results were contradictory to my hypothesis and the results drawn from the previous chapter, and suggest that realised climatic niche shifts are a predominant driver of trait change through time in the introduced range. However, it is possible that the combined noise and the mismatch in scales that may arise when attempting to combine predictions of trait change through time from herbarium 
specimens with niche shifts from correlative SDMs, could mask any underlying patterns in plant trait responses to the new environment. I outline possible approaches that may be better suited to answering this question later in this chapter.

Further research into one of the species included in this dataset (Arctotheca populifolia), revealed distinct morphological trait differences between native and introduced populations, when grown in common garden glasshouse conditions (Brandenburger et al. 2017). I then tested for a difference in plasticity between the native source and introduced populations in response to nutrient and water treatments. Contrary to expectations (Kaufman \& Smouse 2001; Bossdorf et al. 2008; Hylgaard \& Brix 2012), I found a significant overall loss of phenotypic plasticity in the introduced populations of $A$. populifolia, relative to their native source population in as little as 80 years since its introduction. This could have been driven by selection towards reduced plasticity in the introduced range, or due to founder effects upon colonisation. This is the first study to show a rapid decline in phenotypic plasticity over such a short timescale in populations of an introduced plant, relative to their native source population.

Overall differences in phenotypic plasticity between ranges were assessed across a suite of traits using meta-analysis. The approach was adapted from Davidson et al. (2011), calculating a difference between a modified version of two Hedges $d$ effect sizes. Simply calculating a difference between two Hedges d's $\left(P_{\text {diff }} v 1\right)$, as in Davidson et al. (2011), can result in a bias towards overestimating plasticity in the group that has a lower standard deviation. This may tend to overestimate plasticity in introduced populations if they have reduced phenotypic trait variation, if genetic variation is lost on introduction. I propose an updated method that involves pooling the standard deviation between ranges $\left(P_{\text {diff- } v 2)}\right)$. This gives a far more reliable effect size for the difference in plasticity between two groups (i.e., native vs introduced populations). A re-examination of the data and results gathered in Davidson et al. (2011) using this updated method is recommended for further investigation.

In a case study examination into $A$. populifolia, I found that morphological traits (height, leaf area, leaf shape) were significantly different between native and introduced populations. In 
contrast, these differences were not well reflected in trait change through time in herbarium specimens. This suggests that measures of herbarium specimen may not always be a reliable method to predict trait change through time in introduced species. Although some of the differences in mean trait values between ranges, measured across high and low water treatments in controlled glasshouse conditions, did match what would be expected with a shift into a wetter climate (i.e., reduced leaf shape complexity, increase in plant height, reduced leaf senescence when water is limiting), others traits did not (i.e., leaf area would be expected to increase, yet it actually showed a decrease). This could be due to other abiotic or biotic factors, which were not captured by the modelled climate niche envelopes, differing between ranges. Alternatively stochastic founder effects could also have played a key role in the introduction of $A$. populifolia, resulting in differences in morphology, physiology and plasticity between ranges.

\subsection{Research considerations}

\subsubsection{Meta-analysis approach to surmise different groups in single study}

In two of the chapters in this thesis I used a meta-analysis approach to give a summary effect size across different groups within the study. This can be an effective way to synthesize different groups within a study, give an overview of their results, and give a single overall effect size in addressing a hypothesis. Similar approaches have been used to combine results within studies that examine plant responses to warming across multiple experimental sites (Arft et al. 1999; Walker et al. 2006), or to increases in $\mathrm{CO}_{2}$ across multiple years (Ainsworth et al. 2003). The use of a meta-analysis to summarise an overall effect size within studies that use multiple species, multiple sites, or long term experiments are becoming increasingly common in plant ecology (Gurevitch 2013).

Meta-analysis offers clear advantages in comparison to commonly used alternatives, such as vote counting of results. An effect size with confidence intervals gives an estimate of the magnitude and precision of an effect, rather than focusing on testing the significance of a null- 
hypothesis (Gurevitch \& Hedges 1999; Nakagawa \& Cuthill 2007). This allows for different groups to be weighted according to their variance (i.e., their reliability), as well as allowing for the partitioning of within- and between-group variation (Gurevitch 2013). Summary effect sizes allow for more robust results with clearer conclusions, than a narrative summary comparing the results of the different groups (Gurevitch 2013). Furthermore, without a given summary effect, an ad hoc summary of the results (e.g., vote counting) can be more open to interpretation and thus lead to misleading conclusions being made (Borenstein et al. 2009c). The use of meta-analyses within individual studies can be an effective approach to summarise results with an overall effect size, and give clearer answers that will likely continue to be used to address new questions in the future. Nonetheless, I also identified some potential issues that can arise when attempting to compare a difference in phenotypic plasticity between native and introduced ranges (i.e., an effect size of an effect size). Care should be taken as metaanalysis approaches become more common in ecology to synthesise data and address questions in large scale studies.

\subsubsection{Herbarium specimens}

Herbarium specimens can be used to generate historic 'time series data' that can provide a unique insight into how species may be changing in their introduced range (Buswell et al. 2011; Flores-moreno et al. 2014; Dalrymple et al. 2015), in response to human disturbance (McGraw 2001; Law \& Salick 2005) or to climate change (Calinger et al. 2013). However, there can be a lot of potential noise when measuring trait change from herbarium specimens. I used herbarium specimens to model trait change through time in introduced species, and compared this to their shift in realised niche. A key problem with this approach, is that while detecting significant results may make it plausible to then reject the null hypothesis (i.e., climate niche shifts drive trait change through time), detecting non-significant results does not necessarily mean that the null hypothesis can be accepted (i.e., climate niche shifts do not drive trait change in the introduced range). Classical hypothesis testing controls for the type I error (false positive), while the likelihood of a type II error (false negative) is subsequently dependent on 
the sample size and inherent variability. Consequently, statistical artefacts such as small sample size or noisy data can make a type II error much more likely (Vadillo et al. 2016).

In Chapter 4 I did not find evidence for niche shifts relate to multi-generational trait change through time in introduced plants. However, this does not necessarily mean that a shift in the realised climate niche does not influence plant traits. A key issue was the combined noise inherent to both the trait change and niche shift measurements. This is, in part, due to both the predictor and response variables being generated from model outputs, which may themselves be subject to a substantial degree of error or uncertainty. Thus when formulating research questions, care should be taken to minimize noise as much as possible in the variables selected, particularly if they are generated from a model output. If using either a predictor or response variable that has been quantified from a model with substantial likelihood for variation (e.g., predicted niche shifts, trait change through time), I would recommend that the other variable selected is based on using real biological measures (e.g., measured trait differences).

\subsubsection{Quantifying niche shifts}

The approach I used to quantify niche shifts in this study is a fairly simple presence-only method, recommended as a tool to measure ecological niche overlap (Broennimann et al. 2012). It was calculated using a kernel smoother on occurrence densities over two axes in climate space. However, most studies incorporate more climate variables by using the first two axes of principal component analysis of multiple climate variables (Petitpierre et al. 2012; Tingley et al. 2014; Kumar et al. 2015). This has the benefit of accounting for multiple climate variables that may limit species distributions. In the case of Chapter 3 of this thesis I opted to use only two climate variables to predict niche shifts along each axis. This allowed me to make a more meaningful prediction of the rate of trait change through time in response to a niche shift along a specific climate axis (i.e., did a shift into a drier climate coincide with a decline in plant height through time?). Although, I recently found that the use of kernel density estimation 
to quantify niche overlap has been adapted so that it can be applied to more than two dimensions in climate space (Blonder et al. 2014; Tingley et al. 2014).

The use of a kernel smoothing algorithm in climate space is an effective approach to quantify niche shifts. However, it does not generate climate suitability scores that predict the likelihood of a species occurrence in a given environment. Conventional SDM algorithms are still required to predict suitability scores into geographic space. Petitpierre et al. (2012) found that the same niche shift metrics used in this study (niche expansion, total niche change and Schoener's D), correlated with model evaluation scores (Boyce Index) of conventional SDM algorithms (e.g., GLM, MaxEnt), with models that were trained in the native range and predicted into the introduced range. In other words, niche shift metrics generated from a kernel smoothing algorithm of presence points in climate space correlated with the evaluation scores of SDMs generated from a species' native distribution and then predicted into their introduced range (i.e., niche shifts detected using reciprocal SDMs). This suggests that these different approaches can give similar results when used to quantify niche shifts.

\subsection{Recommendations for improving SDM research}

A large amount of the published SDM literature tackles detailed methodological issues, such as how to optimally generate pseudo absences (Barbet-Massin et al. 2012; Senay, Worner \& Ikeda 2013) or minimising sampling bias (Phillips et al. 2009; Syfert, Smith \& Coomes 2013). However, these do not fully deal with some of the key issues that can arise when creating correlative SDMs. With so much research effort directed towards refining modelling approaches, or critiquing the quality of the data and/or assumptions made in the methods, there has been a lack of focus in evaluating the biological meaning and thus actual reliability of SDM outputs (Wittmann et al. 2016).

Conventional presence-only or presence/pseudo-absence SDMs are typically evaluated on a subset of the presence (and pseudo-absence) data, to give an evaluation score that reflects the accuracy of the model predictions on the subsetted data (e.g., the area under curve; 
Fielding \& Bell 1997). However, this may not always be a good indicator of how accurate the model truly is, as it is highly sensitive to the reliability of the input data. Thus I recommend two further approaches that could be used to evaluate correlative SDMs.

\subsubsection{Check SDM predictions in climate space}

When creating a correlative SDM for any purpose, I would recommend plotting the predicted suitability scores into climate space and visually assessing how well the predicted climate niche envelope fits the presence and absence/pseudo absence data (Figure 25). As various combinations of climate conditions will vary in their frequency of occurrence in geographic space, or sometimes not exist at all, there may be large areas of climate space for which there is no presence or pseudo-absence data. Consequently SDM models that are used to predict suitability into unsampled climate space may over or under-predict their climate niche suitability (Warren et al. 2008). Thus, the climate space available to draw absences or pseudoabsences from can have an especially large influence on the predicted suitability scores the model will generate.

If there is a lack of background climate space on the edge of a species' environmental niche (e.g., if the species occurs at the environmental limits available to it), then there will be no climate space to draw absences or pseudo absences from outside of the niche space it occupies (Figure 25B). These missing absences are necessary to describe where the species does not occur in SDM algorithms, in order to determine where the species niche limits will be. This can lead to large over-predictions of adequate suitability into climate space where there is no real occurrence data for the species (i.e., potential false positive/type 1 errors in predictions). Although this may not be a problem when predicting the suitability of habitats in the same climate space that the training data for the model was taken, it can lead to overpredictions of suitability when attempting to project the model into a new range with novel climate conditions, or under future climate change scenarios. 
The accuracy of SDMs are usually evaluated using a subset test dataset of species presences and absences. The sensitivity (true positive rate) and specificity (true negative rate) of the model predictions relative to the test data is then assessed to determine the discriminatory capacity of the SDM (Jimenéz-Valverde 2011). However, as this method of evaluation does not account for the over-extrapolation of predicted suitability into un-sampled climate space, it may erroneously indicate a highly accurate model. By checking the SDM's suitability predictions in climate space, one can be sure that the predictions of the model being used actually 'make sense', and accurately reflect the occurrence data that they are based on. Alternatively, if it is not possible to obtain background absence/pseudo absence data that encompasses the niche space of the presence data (e.g., Figure 25A), then I would recommend using a more simple presence-only SDM algorithm so as to avoid this issue.

\subsubsection{Evaluate SDMs with real biological data}

Another measure to evaluate SDMs would be to compare how well the predicted suitability scores reflect real biological data, such as measures of plant performance, physiological requirements or local scale measures of plant cover (Vaughn \& Omerod 2003; Kearney \& Porter 2009). These approaches are data intensive, and thus may not be appropriate for all exercises involving correlative SDMs. However, this could be a useful avenue of research to see how well suitability scores generated from SDMs based on species occurrence data actually reflect the ecology/biology of the species they are predicting. Despite being data intensive, there is still a lot of potential for the use of SDM approaches in asking novel research questions in regards to a species' performance and its predicted climate niche. 


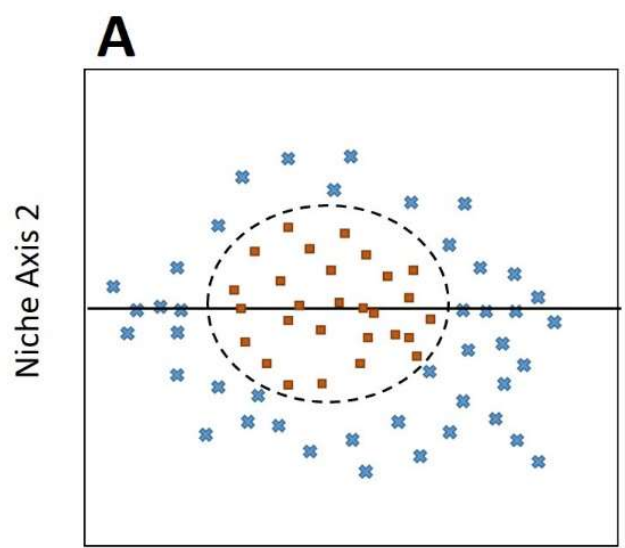

Niche Axis 1

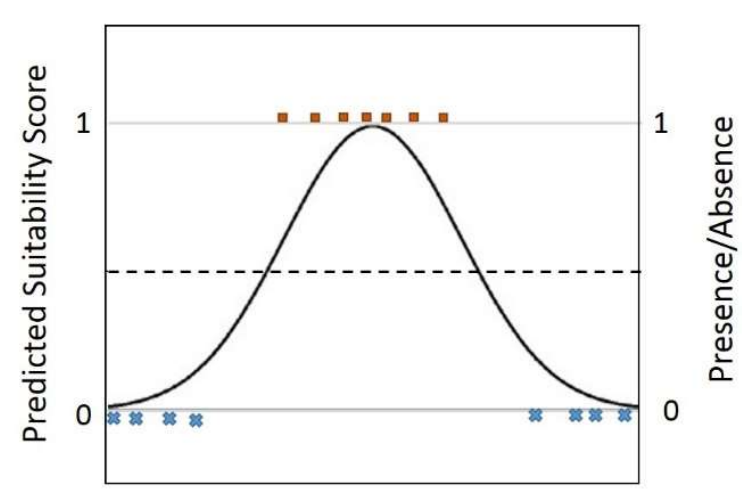

Niche Axis 1

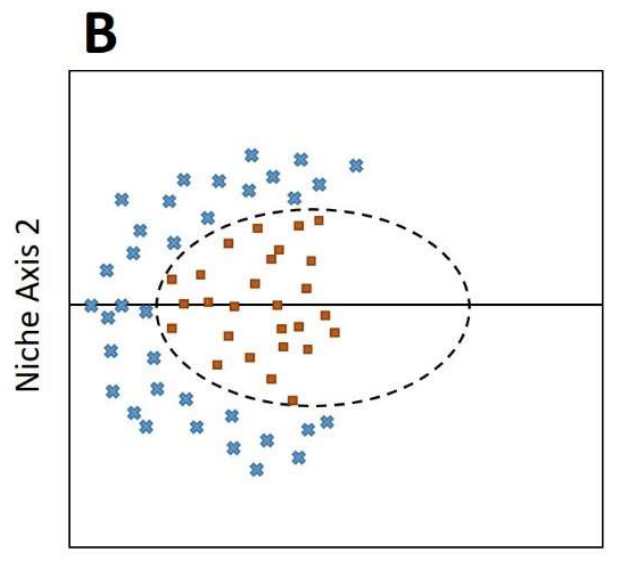

Niche Axis 1

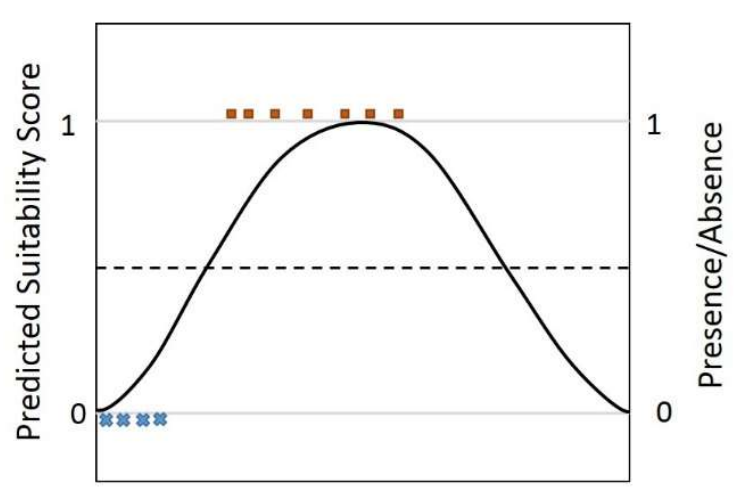

Niche Axis 1

Figure 25. Example of how a lack of absence or pseudo absence data on one edge of a niche axis can create unreliable predictions of suitability scores. This is based on a hypothetical SDM (such as a GLM; logistic regression with quadratic terms for climate variables), that predicts presence occurrence points against absence or pseudo absence points. The top panels show the predicted niche envelope in climate space. The bottom panels show a cross section of this climate niche along one niche axis, indicating how the suitability score is predicted from presence occurrences (1's) against absences/pseudo absences (0's). The orange squares represent presence points and the blue crosses indicate absence/pseudo absence points. The solid line represents a cross section of predicted suitability scores through niche axis 1 . The dashed line represents the predicted threshold of the climate niche envelope (i.e., the realised niche limit). A shows a scenario where absence/pseudo absence points encompass the presence points in climate space, resulting in a niche envelope that accurately reflects where the species does and does not occur. B shows a scenario where there is a lack of presence or absence data points on one edge of niche axis 1, resulting in over prediction into this climate space, for which there is no actual data. This could result in potential false positives (type I errors) when predicting suitability into geographic regions where this climate space does exist (e.g., predicting into a new range or under projected climate change scenarios). 


\subsection{Recommendations for future SDM related research questions}

The increasing availability of large online databases of species traits (TRY, Kattge et al. 2012) and distributions (GBIF, http://www.gbif.org/) has allowed for large global scale studies to be carried out; such as exploring global scale variation in plant traits in relation to climate (Moles et al. 2009; Moles et al. 2011) and for modelling species distributions. Both of these approaches have shown climate to be key in driving species traits and their distributions at the global scale. However little research has gone into synthesizing a clear overview that combines the potential relationships between climate, species distributions, and traits.

Populations that occur in closer proximity to their niche centroid in climate space have been found to have greater genetic diversity (Lira-Noriega \& Manthey 2014) and abundance (Martinez-Meyer et al. 2012). This suggests that the characteristics of a population may be predictable from their location in climate space, in relation to the niche centroid. An alternative approach would be to use SDMs to predict suitability scores for each population, rather than the Euclidian distance of each population to the niche centroid in climate space. Predicted suitability would likely be more appropriate for niche envelopes with greater shape complexity along climate axes. If the predicted suitability scores are interpreted as the likelihood/suitability of that location for the growth/occurrence of a given species, then key functional traits may be expected to correlate with these scores (Figure 26). 


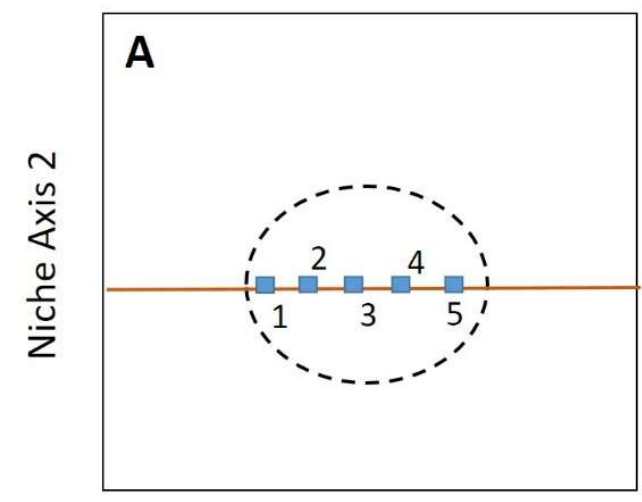

Niche Axis 1

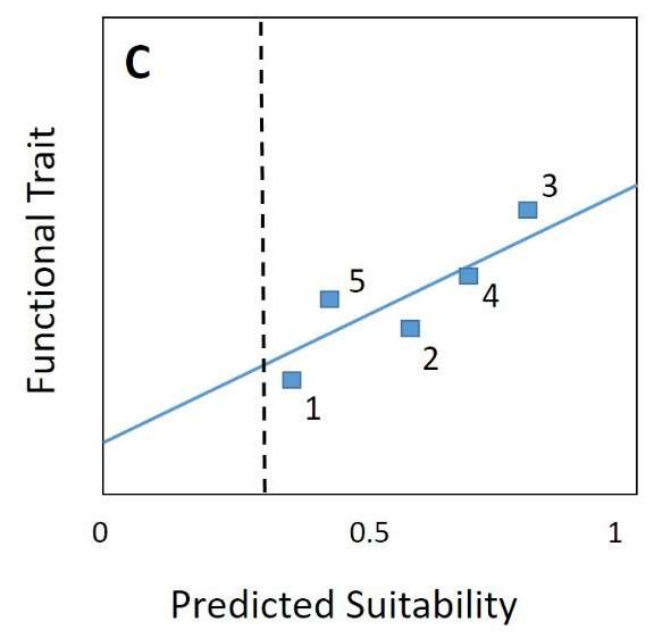

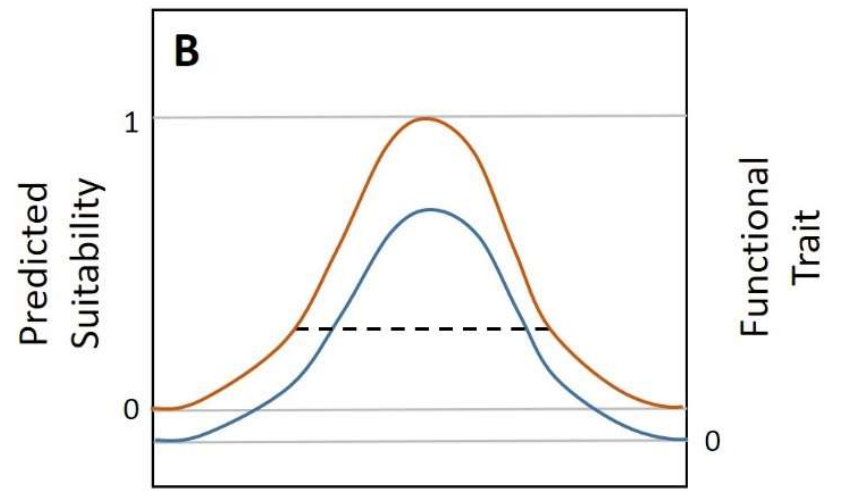

Niche Axis 1

\section{Legend}

- Site locations for trait measurements

- Predicted suitability along a cross section of Niche Axis 1

- Predicted functional trait value

--. Threshold of realised niche

Figure 26. The hypothetical relationship between climate niche space, predicted habitat suitability and plant functional traits. A shows the predicted climate niche envelope, along with the location of sample sites for trait measurements in climate space. B shows a cross section of the climate envelope along niche axis one, with a hypothetical relationship between predicted suitability and functional trait values along this niche axis. $\mathbf{C}$ shows the predicted relationship between habitat suitability and functional trait that would indicate plant fitness (e.g., biomass, reproductive output), with measurements taken from sites along a gradient of habitat suitability. 
Thuiller et al. (2009) used habitat suitability scores generated from correlative SDMs to predict variation in key functional traits along environmental gradients for 16 alpine plant species. They found mixed results, with predicted habitat suitability correlating with functional traits for some species, but not for others. While this provides highly interesting and informative results, studies such as this are very labour intensive as they require both the collection of field data for multiple species at multiple sites, and the creation of SDMs for each of the species. An effective alternative would be to use a meta-analysis approach to calculate effect sizes from glasshouse or common garden experiments, along with generating climate suitability scores for the site locations of the experiment (for common garden experiments), or the origin of population in glasshouse experiments. These scores could then be incorporated as predictor variables in a mixed effects meta-analysis (meta-regression) to see how trait values, or differences in trait values, correspond to predicted habitat suitability scores. This approach would provide a platform from which a number of exciting questions that could be asked:

\subsubsection{Do habitat suitability scores predict variation in key functional traits?}

If the goal of SDMs is to accurately predict the suitability of a species occurrence at a given site, then it would be expected that a species would have greater fitness at sites with a high predicted suitability of occurrence, compared to those predicted to have low suitability (Guisan \& Thuiller 2005). In plants, fitness can be inferred either directly or indirectly by relatively straightforward measures of functional traits (e.g., biomass; Violle et al. 2007). A literature review performed by Wittmann et al. (2016) found 4 published studies assessing this question on various organisms, with 19 out of 22 of the species showing a significant positive or negative relationship between predicted habitat suitability and functional trait. This suggests that SDM habitat suitability scores could be a good indicator of functional traits and/or fitness. However, the mixed direction of trait responses to habitat suitability (both positive and negative) and the vote-counting method of assessing the significant results confuses the overall message. A meta-analysis could be used to assess this further, incorporating additional 
studies to obtain an overall effect size for the prediction of functional traits in in relation to habitat suitability scores, across multiple species.

Studies that examine trait variation at sites across an environmental gradient, or in multiple garden environments across a landscape could be included in a meta-analysis to assess the relationship between predicted habitat suitability and functional traits. With the correlation coefficient or regression slope of a functional trait predicted from habitat suitability scores used as an effect size for each species. This would then allow an overall effect size for a functional trait (e.g., height, biomass, reproductive output) to be obtained. This approach has the added benefit in that it can include multiple studies, in which the primary research question is not directly associated with the proposed hypothesis for the study. This should help to minimize any publication bias in studies used, reducing potential for associated 'file drawer' problems (Rosenthal 1979). Research of this nature would be a key step forward in evaluating the biological interpretation and reliability of SDM outputs.

\subsubsection{Does the magnitude of realised niches shift correspond to trait differences between native and introduced range populations?}

In Chapter 4 of this thesis, I asked if climate niche shifts could predict trait change through time in introduced species. I did not find evidence for trait change through time to coincide with climate niche shifts, however I was unable to conclude if a shift in the realised climate niche does not influence trait change in the introduced range. Furthermore, I did observe some evidence for trait differences between native and introduced populations of $A$. populifolia to correspond with what would be expected following a shift into wetter climate in the introduced range (Chapter 7 ). Following on from this, another question that could be asked is; do realised niche shifts predict trait differences between native and introduced range populations? This is a similar question to that asked in Chapter 4 of this thesis; the key difference being that the effect size would be the difference in trait values between native and introduced populations, indicating the degree of trait divergence between ranges. 
The large number of studies that examine trait differences between native and introduced ranges in controlled glasshouse or common garden experiments (Genton et al. 2005; Williams et al. 2008; Colautti et al. 2009; Ebeling et al. 2011), provide an opportunity for a meta-analysis to be conducted, which asks if niche shifts predict trait divergence between ranges? A possible approach would be to use the difference in trait values between native and introduced populations as an effect size in a meta-regression, which would then be predicted by the difference in habitat suitability scores between the native and introduced range sites, from a SDM that has been trained on the species' native range. The model formula would be:

$$
\left(T_{n}-T_{i}\right) \sim\left(S_{n}-S_{i}\right)
$$

(equation 8.)

Where; $\quad T_{n}=$ Mean trait value of the native population

$T_{i}=$ Mean trait value of the introduced population

$S_{n}=$ The habitat suitability score of the native range site

$S_{i}=$ The habitat suitability score of the introduced range site

By creating a SDM using training data from just the native range, the magnitude of niche shift a population has undergone could be inferred by predicting habitat suitability for the introduced range sites. The difference in the habitat suitability scores between a native and introduced range site would represent the magnitude of the niche shift that the introduced site has undergone, in relation to the native range site with which it is being compared. Thus it would be expected that the greater the difference in predicted climate suitability between sites, the greater the difference in trait values.

Testing if niche shifts predict trait divergence between ranges would have a number of advantages over the approach I used with herbarium specimens in Chapter 3, in that: i) there would be less noise in the measurement of trait differences (i.e., these measurements should be a more accurate reflection of the actual populations), ii) habitat suitability scores could be applied to the location of each population, thus accounting for some of the potential mismatch in spatial-scale that could arise from predicting trait differences in populations from niche shifts calculated from global occurrence data, and iii) the large number of published studies 
examining trait differences between native and introduced ranges would allow for a large number replicates to be included in the meta-regression.

\subsubsection{Does phenotypic plasticity decrease on niche margins?}

Glasshouse or common garden experiments often involve growing populations in different environmental conditions. This also enables plasticity to be assessed in many studies, even if that was not the study's primary goal (Davidson et al. 2011). Phenotypic plasticity has been hypothesized to be lower in marginal range edge populations, due to higher levels of environmental stress (Magi et al. 2011) or because these populations may be smaller with less genetic diversity (Berg et al. 2005). Thus, plasticity may also be expected to be lower in populations on the margins of its environmental niche (i.e., those with lower predicted suitability), relative to more central populations.

Phenotypic plasticity in a population can be assessed from studies that use common garden or glasshouse experiments to measure traits across multiple environments. The difference in trait values between treatments could be used as an effect size for each population. SDMs would be generated from occurrence data for each species in the study. Habitat suitability scores would then be predicted for the site of each population. This would allow for a metaregression to be performed, predicting phenotypic plasticity in response to habitat suitability score.

\subsubsection{Does habitat suitability predict species' local abundance?}

Another biologically meaningful way to validate the accuracy of species distribution models could be to look at habitat suitability scores in relation to their true percentage cover in real field sites. If the goal of species distribution models is to predict the suitability of a site for a species, indicating the likelihood of its occurrence, then local percentage cover may be expected to reflect this. Large landscape scale studies that take measurements of plant percentage cover provide an opportunity to examine this. Stevens et al. (2011) provides a 
freely available online dataset for species cover compositions for 153 grassland sites across Europe. Similarly, the New Zealand National Vegetation Survey databank (NVS; Wiser et al. 2001) and Land Use and Carbon Analysis System (LUCAS; Coomes et al. 2002) forest plot data provide data of percentage cover and biomass estimates for thousands of plots throughout New Zealand. These datasets could be used to obtain percentage cover at each site, for species that frequently re-occur in these plots. Species distribution models could then be created for each species to generate habitat suitability scores for each site. If SDM suitability scores do reflect a species' likelihood of prevalence at the microsite scale, then greater suitability scores would be expected to coincide with increasing percentage cover.

A key advantage of this study would be that when discrepancies arise (i.e., habitat suitability does not predict percentage cover), the possible causes for this can then be investigated further. The Stevens et al. (2011) dataset also includes information on soil conditions, nitrogen deposition, management and the prevalence of other species at the site. Each of these factors may limit a species' cover at a given site, even if it is predicted to have high habitat suitability score. Validating SDMs that have been created using large continental scale species distribution data, with microsite scale measurements of a species' actual occurrence, would provide a unique insight into how effective SDMs are in reflecting real biological processes.

\subsection{Personnel reflections and recommendations}

From my experiences in researching this thesis, a key recommendation I would make is to take special care in ensuring that any model predictions or calculated indices appropriately reflect the data that they are based on. In two separate incidents in my research, I followed approaches that have been used in prior studies, using what appear to be sound methods at first glance. However, on further inspection I found that the output of these approaches did not correspond appropriately to the data they were based on.

In my initial research for Chapter 4 , I found how a lack of available climate space to draw presence or absence values from, can lead to SDMs over-predicting suitability into 
'undocumented' climate space, which I discuss earlier in this chapter. This issue is not well detected using conventional model evaluation techniques (e.g., AUC score), thus I recommend inspecting SDM model predictions in climate space: i) to check for biologically unreasonable response curves or suitability contours, and ii) because this allows for predicted suitability scores to be inspected in relation to the presence and absence data used to generate them, and ensure that their predictions are not being extrapolated into climate space that lacks input data.

In chapter three I followed a method used in Davidson et al. (2011) to calculate 'an effect size of an effect size', for a difference in plasticity between native and introduced populations. Although the method appeared sound, it wasn't until closer examination of individual effect sizes for each trait did it become apparent that some of these did not reflect the true differences in plasticity between ranges (outlined in Chapter 6). These are two examples of erroneous calculations that could have easily been missed if I hadn't been especially thorough in checking my methods and result, and critical enough to recognize the possibility of some fundamental flaws in published research. As a PhD student, I would recommend to any early career scientists or research students to be especially thorough in the checking of their methods, results, and any assumptions made in their research approach. In addition to trusting ones intuition when feeling there is something amiss with other published research. 


\section{$\underline{8.5 \text { Concluding remarks }}$}

In this thesis, I assessed how introduced plants may respond to a change in environment via rapid evolution in key morphological traits, or via the rapid evolution of phenotypic plasticity. My key conclusions from this study are:

The magnitude and direction of realised climate niche shifts did not predict trait change through time in introduced plants. However, the combined noise and uncertainty associated with the two approaches (i.e., modelling trait change through time from herbarium specimens and quantifying climate niche shifts) make it difficult to draw clear conclusions as to the relation between a change in climate and adaptation in a species' introduced range. Nevertheless, a common garden glasshouse experiment with Arctotheca populifolia did reveal some distinct trait differences between ranges that were not detected from analysis of herbarium specimens. Some of these trait differences did correspond to what might be expected following a shift into wetter climate (e.g., reduced leaf shape complexity, increase in height, reduced leaf senescence). Therefore, trait differences between native and introduced populations, compared in glasshouse or common garden experiments, may be a better tool to assess trait divergence in relation to a shift in realised niche. Glasshouse and/or common garden experiments can be both time consuming and expensive to run. This can pose a challenge for running a multi-species assessment. However, the large amount of already published data make meta-analysis a viable approach to assess trait divergence between ranges, in relation to a realised niche shift. Irrespective of this proposed study, the use of common garden and/or glasshouse experiments should remain an important tool to evaluate the reliability of modelling predictions.

Contrary to my expectations, Arctotheca populifolia underwent a rapid loss of phenotypic plasticity in as little as 80 years since its introduction to Eastern Australia. Additionally, I demonstrate that the method used to calculate an effect size for the difference in overall phenotypic plasticity between native and invasive populations in a meta-analysis (Davidson et al. 2011), has a bias towards overestimating plasticity in the population with lower standard 
deviation. Thus, the assumption that phenotypic plasticity is always beneficial and tends to increase in introduced/invasive species may need to be reconsidered and tested further.

As scientific research continues to focus on the capability to publish answers to novel research questions, it is likely that novel methods will be employed to address them. The increasing availability of large datasets of plant traits, species distributions, climate conditions, and previously published research, will allow for exciting and novel 'big data' research to be carried out. This may also coincide with a growth of complex and novel research methods. However, any assumptions in these procedures should be carefully considered, with particular care taken to ensure that model predictions reliably reflect the real world data that they represent. 


\section{References}

Ackerly, D. D. (2003) Community assembly, niche conservatism, and adaptive evolution in changing environments. International Journal of Plant Sciences, 164, S165-S184.

Adamson, D. \& Adamson, H. (1999) A southern hemisphere view of nature. Plants in action: adaptation in nature, performance in cultivation (ed. by B.J. Atwell, P. Kriedemann and C. Turnbull), pp. 8-10. Macmillan Education AU, South Yarra.

Ahmadikhah, A. \& Marufinia, A. (2016) Effect of reduced plant height on drought tolerance in rice. 3 Biotech, 6, 221.

Ainsworth, E.A., Davey, P.A., Hymus, G.J., Osborne, C.P., Rogers, A., Blum, H., NÖSberger, J. \& Long, S.P. (2003) Is stimulation of leaf photosynthesis by elevated carbon dioxide concentration maintained in the long term? A test with Lolium perenne grown for 10 years at two nitrogen fertilization levels under Free Air $\mathrm{CO}_{2}$ Enrichment (FACE). Plant, Cell \& Environment, 26, 705-714.

Akaike, H. (1998) Information hheory and an extension of the maximum likelihood principle. Selected papers of Hirotugu Akaike (ed. by E. Parzen, K. Tanabe and G. Kitagawa), pp. 199-213. Springer New York, New York, NY.

Alexander, J.M. \& Edwards, P.J. (2010) Limits to the niche and range margins of alien species. Oikos, 119, 1377-1386.

Alpert, P. \& Simms, E.L. (2002) The relative advantages of plasticity and fixity in different environments: when is it good for a plant to adjust? Evolutionary Ecology, 16, 285-297.

Anderson, J.T., Willis, J.H. \& Mitchell-Olds, T. (2011) Evolutionary genetics of plant adaptation. Trends in Genetics, 27, 258-266.

Araújo, M.B., Thuiller, W. \& Pearson, R.G. (2006) Climate warming and the decline of amphibians and reptiles in Europe. Journal of Biogeography, 33, 1712-1728.

Arft, A.M., Walker, M.D., Gurevitch, J., Alatalo, J.M., Bret-Harte, M.S., Dale, M., Diemer, M., Gugerli, F., Henry, G.H.R., Jones, M.H., Hollister, R.D., Jónsdóttir, I.S., Laine, K., Lévesque, E., Marion, G.M., Molau, U., Mølgaard, P., Nordenhäll, U., Raszhivin, V., Robinson, C.H., Starr, G., Stenström, A., Stenström, M., Totland, Ø., Turner, P.L., Walker, L.J., Webber, P.J., Welker, J.M. \& Wookey, P.A. (1999) Responses of tundra plants to experimental qarming: meta-analysis of the international tundra experiment. Ecological Monographs, 69, 491-511.

Ashley, M.V., Willson, M.F., Pergams, O.R.W., O'Dowd, D.J., Gende, S.M. \& Brown, J.S. (2003) Evolutionarily enlightened management. Biological Conservation, 111, 115123.

AVG Database (2014) Australia's Virtual Herbarium. Available at http://avh.ala.org.au (accessed 8 August 2013). 
Baher, Z.F., Mirza, M., Ghorbanli, M. \& Bagher Rezaii, M. (2002) The influence of water stress on plant height, herbal and essential oil yield and composition in Satureja hortensis L. Flavour and Fragrance Journal, 17, 275-277.

Baker, H.G. (1965) Characteristics and modes of origin of weeds. The Genetics of Colonizing Species (ed. by H.G. Baker and G.L. Stebbins), pp. 147-169. Academic Press, New York.

Barbet-Massin, M., Jiguet, F., Albert, C.H. \& Thuiller, W. (2012) Selecting pseudo-absences for species distribution models: how, where and how many? Methods in Ecology and Evolution, 3, 327-338.

Bastlová, D. \& Květ, J. (2002) Differences in dry weight partitioning and flowering phenology between native and non-native plants of purple loosestrife (Lythrum salicaria L.). Flora, 197, 332-340.

Becker, B.J. \& Wu, M.-J. (2007) The synthesis of regression slopes in meta-analysis. Statistical Science, 22, 414-429.

Berg, H., Becker, U. \& Matthies, D. (2005) Phenotypic plasticity in Carlina vulgaris: effects of geographical origin, population size, and population isolation. Oecologia, 143, 220231.

Berg, M.P. \& Ellers, J. (2010) Trait plasticity in species interactions: a driving force of community dynamics. Evolutionary Ecology, 24, 617-629.

Blonder, B., Lamanna, C., Violle, C. \& Enquist, B.J. (2014) The n-dimensional hypervolume. Global Ecology and Biogeography, 23, 595-609.

Blossey, B. \& Notzold, R. (1995) Evolution of increased competitive ability in invasive nonindigenous plants: a hypothesis. Journal of Ecology, 83, 887-889.

Bocsi, T., Allen, J.M., Bellemare, J., Kartesz, J., Nishino, M. \& Bradley, B.A. (2016) Plants' native distributions do not reflect climatic tolerance. Diversity and Distributions, 22, 615-624.

Bolnick, D.I., Svanbäck, R., Araújo, M.S. \& Persson, L. (2007) Comparative support for the niche variation hypothesis that more generalized populations also are more heterogeneous. Proceedings of the National Academy of Sciences of the United States of America, 104, 10075-10079.

Borenstein, M., Hedges, L.V., Higgins, J.P.T. \& Rothstein, H.R. (2009a) Complex data structures. Introduction to Meta-Analysis, pp. 213-216. John Wiley \& Sons, Ltd.

Borenstein, M., Hedges, L.V., Higgins, J.P.T. \& Rothstein, H.R. (2009b) Effect szes based on means. Introduction to Meta-Analysis, pp. 21-32. John Wiley \& Sons, Ltd.

Borenstein, M., Hedges, L.V., Higgins, J.P.T. \& Rothstein, H.R. (2009c) Why perform a metaanalysis. Introduction to Meta-Analysis, pp. 9-14. John Wiley \& Sons, Ltd. 
Bossdorf, O., Lipowsky, A. \& Prati, D. (2008) Selection of preadapted populations allowed Senecio inaequidens to invade Central Europe. Diversity and Distributions, 14, 676685.

Bossdorf, O., Auge, H., Lafuma, L., Rogers, W.E., Siemann, E. \& Prati, D. (2005) Phenotypic and genetic differentiation between native and introduced plant populations. Oecologia, 144, 1-11.

Botto, J.F. \& Smith, H. (2002) Differential genetic variation in adaptive strategies to a common environmental signal in Arabidopsis accessions: phytochrome-mediated shade avoidance. Plant, Cell \& Environment, 25, 53-63.

Box, G.E.P., Jenkis, G.M. \& Reinsel, G.C. (2007) Time series analysis: forcasting and control, Fourth edn. John Wiley \& Sons, Hoboken, New Jersey.

Bradshaw, A.D. (1965) Evolutionary Significance of Phenotypic Plasticity in Plants. Advances in Genetics, 13, 115-155.

Bradshaw, A.D. (1984) Ecological signifcance of genetic variation between populations. Perspectives on Plant Populations Ecology (ed. by R. Dirzo and J. Sarukhán), pp. 213218. Sinauer, Sunderland, MA.

Brambilla, M. \& Ficetola, G.F. (2012) Species distribution models as a tool to estimate reproductive parameters: a case study with a passerine bird species. Journal of Animal Ecology, 81, 781-787.

Brandenburger, C.R., Sherwin, W.B., Creer, S., Buitenwerf, R., Poore, A.G.B. \& Moles, A.T. (2017) Rapid reshaping: The evolution of striking morphological changes in an introduced beach daisy. Manuscript submitted for publication,

Broennimann, O., Treier, U.A., Müller-Schärer, H., Thuiller, W., Peterson, A.T. \& Guisan, A. (2007) Evidence of climatic niche shift during biological invasion. Ecology Letters, 10, 701-709.

Broennimann, O., Fitzpatrick, M.C., Pearman, P.B., Petitpierre, B., Pellissier, L., Yoccoz, N.G., Thuiller, W., Fortin, M.-J., Randin, C., Zimmermann, N.E., Graham, C.H. \& Guisan, A. (2012) Measuring ecological niche overlap from occurrence and spatial environmental data. Global Ecology and Biogeography, 21, 481-497.

Burnham, K.P. \& Anderson, D.R. (2003) Model selection and multi model inference: a practical information-theoretic appraoch, Second edn. Springer, New York.

Buswell, J.M. (2009) Rapid evolution in introduced plant species. Victoria University of Wellington, Welllington, New Zealand.

Buswell, J.M., Moles, A.T. \& Hartley, S. (2011) Is rapid evolution common in introduced plant species? Journal of Ecology, 99, 214-224.

Byers, J.E., Smith, R.S., Pringle, J.M., Clark, G.F., Gribben, P.E., Hewitt, C.L., Inglis, G.J., Johnston, E.L., Ruiz, G.M., Stachowicz, J.J. \& Bishop, M.J. (2015) Invasion Expansion: Time since introduction best predicts global ranges of marine invaders. In: Scientific reports, p. 12436 
Calinger, K.M., Queenborough, S. \& Curtis, P.S. (2013) Herbarium specimens reveal the footprint of climate change on flowering trends across north-central North America. Ecology Letters, 16, 1037-1044.

Caño, L., Escarré, J., Fleck, I., Blanco-Moreno, J. \& Sans, F. (2008) Increased fitness and plasticity of an invasive species in its introduced range: A study using Senecio pterophorus. Journal of Ecology, 96, 468-476.

Carneiro, L.R.d.A., Lima, A.P., Machado, R.B. \& Magnusson, W.E. (2016) Limitations to the use of species-distribution models for environmental-impact assessments in the Amazon. PLOS ONE, 11, e0146543.

Carroll, S.P., Hendry, A.P., Reznick, D.N. \& Fox, C.W. (2007) Evolution on ecological timescales. Functional Ecology, 21, 387-393.

Cavender-Bares, J. \& A. Bazzaz, F. (2004) From leaves to ecosystems: using chlorophyll fluorescence to assess photosynthesis and plant function in ecological studies. Chlorophyll a Fluorescence: A Signature of Photosynthesis (ed. by G.C. Papageorgiou and Govindjee), pp. 737-755. Springer Netherlands, Dordrecht.

Chun, Y.J., Collyer, M.L., Moloney, K.A. \& Nason, J.D. (2007) Phenotypic plasticity of native vs. invasive purple loosestrife: a two-state multivariate approach. Ecology, 88, 14991512.

Colautti, R.I. \& Barrett, S.C.H. (2013) Rapid adaptation to climate facilitates range expansion of an invasive plant. Science, 342, 364-366.

Colautti, R.I., Maron, J.L. \& Barrett, S.C. (2009) Common garden comparisons of native and introduced plant populations: latitudinal clines can obscure evolutionary inferences. Evolutionary Applications, 2, 187-199.

Coleman, J.S., McConnaughay, K.D.M. \& Ackerly, D.D. (1994) Interpreting phenotypic variation in plants. Trends in Ecology \& Evolution, 9, 187-191.

Colomer-Ventura, F., Martínez-Vilalta, J., Zuccarini, P., Escolà, A., Armengot, L. \& Castells, E. (2015) Contemporary evolution of an invasive plant is associated with climate but not with herbivory. Functional Ecology, 29, 1475-1485.

Colwell, R.K. \& Rangel, T.F. (2009) Hutchinson's duality: The once and future niche. Proceedings of the National Academy of Sciences, 106, 19651-19658.

Coomes, D.A., Allen, R.B., Scott, N.A., Goulding, C. \& Beets, P. (2002) Designing systems to monitor carbon stocks in forests and shrublands. Forest Ecology and Management, 164, 89-108.

Cornelissen, J.H.C., Lavorel, S., Garnier, E., Díaz, S., Buchmann, N., Gurvich, D.E., Reich, P.B., Steege, H.t., Morgan, H.D., Heijden, M.G.A.v.d., Pausas, J.G. \& Poorter, H. (2003) A handbook of protocols for standardised and easy measurement of plant functional traits worldwide. Australian Journal of Botany, 51, 335-380.

Cox, G.W. (2004) Alien species and evolution: the evolutionary ecology of exotic plants, animals, microbes, and interacting native species. Island Press, Washington DC. 
Crawley, M.J. (1987) What makes a community invasible? Colonization, succession and stability (ed. by A.J. Gray, M.J. Crawley and P.J. Edwards), pp. 429-453. Blackwell Scientific, Oxford.

Crick, J.C. \& Grime, J.P. (1987) Morphological plasticity and mineral nutrient capture in two herbaceous species of contrasted ecology. New Phytologist, 107, 403-414.

Crispo, E., DiBattista, J.D., Correa, C., Thibert-Plante, X., McKellar, A.E., Schwartz, A.K., Berner, D., De León, L.F. \& Hendry, A.P. (2010) The evolution of phenotypic plasticity in response to anthropogenic disturbance. Evolutionary Ecology Research, 12, 47-66.

Cruz-Cárdenas, G., López-Mata, L., Villaseñor, J.L. \& Ortiz, E. (2014) Potential species distribution modeling and the use of principal component analysis as predictor variables. Revista Mexicana de Biodiversidad, 85, 189-199.

Dalrymple, R.L., Buswell, J.M. \& Moles, A.T. (2015) Asexual plants change just as often and just as fast as do sexual plants when introduced to a new range. Oikos, 124, 196-205.

Davidson, A.M., Jennions, M. \& Nicotra, A.B. (2011) Do invasive species show higher phenotypic plasticity than native species and, if so, is it adaptive? A meta-analysis. Ecology Letters, 14, 419-431.

Davis, A.J., Jenkinson, L.S., Lawton, J.H., Shorrocks, B. \& Wood, S. (1998) Making mistakes when predicting shifts in species range in response to global warming. Nature, 391, 783-786.

DeWalt, S.J., Denslow, J.S. \& Hamrick, J.L. (2004) Biomass allocation, growth, and photosynthesis of genotypes from native and introduced ranges of the tropical shrub Clidemia hirta. Oecologia, 138, 521-531.

DeWitt, T.J. \& Scheiner, S.M. (2004) Phenotypic plasticity: functional and conceptual approaches. Oxford University Press, Oxford.

DeWitt, T.J., Sih, A. \& Wilson, D.S. (1998) Costs and limits of phenotypic plasticity. Trends in Ecology \& Evolution, 13, 77-81.

Dlugosch, K.M. \& Parker, I.M. (2008) Founding events in species invasions: genetic variation, adaptive evolution, and the role of multiple introductions. Molecular Ecology, 17, 431449.

Donohue, K., Pyle, E.H., Messiqua, D., Heschel, M.S. \& Schmitt, J. (2001) Adaptive divergence in plasticity in natural populations of Impatiens capensis and Its consequences for performance in novel habitats. Evolution, 55, 692-702.

Dormann, C.F., Schymanski, S.J., Cabral, J., Chuine, I., Graham, C., Hartig, F., Kearney, M., Morin, X., Römermann, C., Schröder, B. \& Singer, A. (2012) Correlation and process in species distribution models: bridging a dichotomy. Journal of Biogeography, 39, 2119-2131.

Ebeling, S.K., Stöcklin, J., Hensen, I. \& Auge, H. (2011) Multiple common garden experiments suggest lack of local adaptation in an invasive ornamental plant. Journal of Plant Ecology, 4, 209-220. 
Eckert, C.G., Manicacci, D. \& Barrett, S.C.H. (1996) Genetic drift and founder effect in native versus introduced populations of an invading plant, Lythrum salicaria (Lythraceae). Evolution, 50, 1512-1519.

Elith, J. \& Leathwick, J.R. (2009) Species distribution models: ecological explanation and prediction across space and time. Annual review of ecology, evolution, and systematics, 40, 677-697.

Elith, J., Ferrier, S., Huettmann, F. \& Leathwick, J. (2005) The evaluation strip: A new and robust method for plotting predicted responses from species distribution models. Ecological Modelling, 186, 280-289.

Elmendorf, S.C. \& Moore, K.A. (2008) Use of community-composition data to predict the fecundity and abundance of species. Conservation Biology, 22, 1523-1532.

Emery, R.J.N., Chinnappa, C.C. \& Chmielewski, J.G. (1994) Specialization, plant strategies, and phenotypic plasticity in populations of Stellaria longipes along an elevational gradient. International Journal of Plant Sciences, 155, 203-219.

Enquist, B.J., Brown, J.H. \& West, G.B. (1998) Allometric scaling of plant energetics and population density. Nature, 395, 163.

Escobar, L.E., Qiao, H., Phelps, N.B.D., Wagner, C.K. \& Larkin, D.J. (2016) Realized niche shift associated with the Eurasian charophyte Nitellopsis obtusa becoming invasive in North America. Scientic Reports, 6, 29037.

Felker-Quinn, E., Schweitzer, J.A. \& Bailey, J.K. (2013) Meta-analysis reveals evolution in invasive plant species but little support for Evolution of Increased Competitive Ability (EICA). Ecology and Evolution, 3, 739-751.

Fitzpatrick, B.M. (2012) Underappreciated consequences of phenotypic plasticity for ecological speciation. International Journal of Ecology, 2012, 12.

Fitzpatrick, M.C., Weltzin, J.F., Sanders, N.J. \& Dunn, R.R. (2007) The biogeography of prediction error: why does the introduced range of the fire ant over-predict its native range? Global Ecology and Biogeography, 16, 24-33.

Flores-Moreno, H., García-Treviño, E.S., Letten, A.D. \& Moles, A.T. (2015) In the beginning: phenotypic change in three invasive species through their first two centuries since introduction. Biological Invasions, 17, 1215-1225.

Flory, S.L., Long, F. \& Clay, K. (2011) Invasive Microstegium populations consistently outperform native range populations across diverse environments. Ecology, 92, 22482257.

Fonseca, C.R., Overton, J.M., Collins, B. \& Westoby, M. (2000) Shifts in trait-combinations along rainfall and phosphorus gradients. Journal of Ecology, 88, 964-977.

Forsman, A. (2015) Rethinking phenotypic plasticity and its consequences for individuals, populations and species. Heredity, 115, 276-284. 
Funk, J.L. (2008) Differences in plasticity between invasive and native plants from a low resource environment. Journal of Ecology, 96, 1162-1173.

Gabriel, W. (2005) How stress selects for reversible phenotypic plasticity. Journal of Evolutionary Biology, 18, 873-883.

Gallagher, R.V., Beaumont, L.J., Hughes, L. \& Leishman, M.R. (2010) Evidence for climatic niche and biome shifts between native and novel ranges in plant species introduced to Australia. Journal of Ecology, 98, 790-799.

Gallien, L., Münkemüller, T., Albert, C.H., Boulangeat, I. \& Thuiller, W. (2010) Predicting potential distributions of invasive species: where to go from here? Diversity and Distributions, 16, 331-342.

Gallien, L., Thuiller, W., Fort, N., Boleda, M., Alberto, F.J., Rioux, D., Lainé, J. \& Lavergne, S. (2016) Is there any evidence for rapid, genetically-based, climatic niche expansion in the invasive common ragweed? PLOS ONE, 11, e0152867.

Genton, B.J., Kotanen, P.M., Cheptou, P.-O., Adolphe, C. \& Shykoff, J.A. (2005) Enemy release but no evolutionary loss of defence in a plant invasion: an inter-continental reciprocal transplant experiment. Oecologia, 146, 404-414.

Getty, T. (1996) The maintenance of phenotypic plasticity as a signal detection problem. The American Naturalist, 148, 378-385.

Ghalambor, C.K., McKay, J.K., Carroll, S.P. \& Reznick, D.N. (2007) Adaptive versus nonadaptive phenotypic plasticity and the potential for contemporary adaptation in new environments. Functional Ecology, 21, 394-407.

Gilbert, M.E. (2008) The zonation of coastal dune plants in relation to sand burial, resource availability and physiological adaptation Doctoral,

Gilbert, S.F. \& Epel, D. (2009) Ecological developmental biology: integrating epigenetics, medicine and evolution. Sinauer, Sunderland, MA.

Godsoe, W. \& Case, B.S. (2015) Accounting for shifts in the frequency of suitable environments when testing for niche overlap. Methods in Ecology and Evolution, 6, 5966.

Gomez-Mestre, I. \& Jovani, R. (2013) A heuristic model on the role of plasticity in adaptive evolution: plasticity increases adaptation, population viability and genetic variation. Proceedings of the Royal Society B: Biological Sciences, $\mathbf{2 8 0}$

Grassein, F., Till-Bottraud, I. \& Lavorel, S. (2010) Plant resource-use strategies: the importance of phenotypic plasticity in response to a productivity gradient for two subalpine species. Annals of Botany, 106, 637-645.

Gratani, L. (2014) Plant phenotypic plasticity in response to environmental factors. Advances in Botany, 2014, 17. 
Grime, J.P. (1977) Evidence for the existence of three primary strategies in plants and its eelevance to ecological and evolutionary theory. The American Naturalist, 111, 11691194.

Guerin, G.R., Wen, H. \& Lowe, A.J. (2012) Leaf morphology shift linked to climate change. Biology Letters, 8, 882-886.

Guisan, A. \& Thuiller, W. (2005) Predicting species distribution: offering more than simple habitat models. Ecology Letters, 8, 993-1009.

Guisan, A., Petitpierre, B., Broennimann, O., Daehler, C. \& Kueffer, C. (2014) Unifying niche shift studies: insights from biological invasions. Trends in ecology \& evolution, 29, $260-$ 269.

Guisan, A., Tingley, R., Baumgartner, J.B., Naujokaitis-Lewis, I., Sutcliffe, P.R., Tulloch, A.I.T., Regan, T.J., Brotons, L., McDonald-Madden, E., Mantyka-Pringle, C., Martin, T.G., Rhodes, J.R., Maggini, R., Setterfield, S.A., Elith, J., Schwartz, M.W., Wintle, B.A., Broennimann, O., Austin, M., Ferrier, S., Kearney, M.R., Possingham, H.P. \& Buckley, Y.M. (2013) Predicting species distributions for conservation decisions. Ecology Letters, 16, 1424-1435.

Gurevitch, J. (2013) Meta-analysis of results from multisite studies. Handbook of Metaanalysis in Ecology and Evolution (ed. by J. Koricheva, J. Gurevitch and K. Mengersen), pp. 313-320. Princeton University Press, Princeton, New Jersey.

Gurevitch, J. \& Hedges, L.V. (1999) Statistical issues in ecological meta-analyses. Ecology, 80, 1142-1149.

Güsewelll, S., Jakobs, G. \& Weber, E. (2006) Native and introduced populations of Solidago gigantea differ in shoot production but not in leaf traits or litter decomposition. Functional Ecology, 20, 575-584.

Hartley, S., Harris, R. \& Lester, P.J. (2006) Quantifying uncertainty in the potential distribution of an invasive species: climate and the Argentine ant. Ecology Letters, 9, 1068-1079.

Hefley, T.J. \& Hooten, M.B. (2016) Hierarchical species distribution models. Current Landscape Ecology Reports, 1, 87-97.

Heidy Kikillus, K., Hare, K.M. \& Hartley, S. (2010) Minimizing false-negatives when predicting the potential distribution of an invasive species: a bioclimatic envelope for the redeared slider at global and regional scales. Animal Conservation, 13, 5-15.

Hesp, P. (2002) Foredunes and blowouts: initiation, geomorphology and dynamics. Geomorphology, 48, 245-268.

Hijmans, R.J. \& Elith, J. (2017) Species distribution modeling with R. R package version 0.811.

Hijmans, R.J., Cameron, S.E., Parra, J.L., Jones, P.G. \& Jarvis, A. (2005) Very high resolution interpolated climate surfaces for global land areas. International Journal of Climatology, 25, 1965-1978. 
Hill, M.P. \& Terblanche, J.S. (2014) Niche overlap of congeneric invaders supports a singlespecies hypothesis and provides insight into future invasion risk: implications for global Management of the Bactrocera dorsalis complex. PLOS ONE, 9, e90121.

Hobbie, S.E. \& Chapin, F.S. (1998) The response of tundra plant biomass, aboveground production, nitrogen, and $\mathrm{CO}_{2}$ flux to experimental warming. Ecology, 79, 1526-1544.

Hoffmann, A.A. \& Sgro, C.M. (2011) Climate change and evolutionary adaptation. Nature, 470, 479-485.

Holt, R.D. (2009) Bringing the Hutchinsonian niche into the 21st century: ecological and evolutionary perspectives. Proceedings of the National Academy of Sciences, 106, 19659-19665.

Holt, R.D., Barfield, M. \& Gomulkiewicz, R. (2005) Theories of niche conservatism and evolution: could exotic species be potential tests? Species invasions: insights into ecology, evolution, and biogeography (ed. by D.F. Sax, J.J. Stachowicz and S.D. Gaines), pp. 259-290. Sinauer, Sunderland, MA, USA.

Hori, M. (1993) Frequency-dependent natural selection in the handedness of scale-eating cichlid fish. Science, 260, 216-219.

Huang, Q.Q., Pan, X.Y., Fan, Z.W. \& Peng, S.L. (2015) Stress relief may promote the evolution of greater phenotypic plasticity in exotic invasive species: a hypothesis. Ecology and Evolution, 5, 1169-1177.

Hulme, P.E. (2009) Trade, transport and trouble: managing invasive species pathways in an era of globalization. Journal of Applied Ecology, 46, 10-18.

Hulme, P.E. \& Barrett, S.C.H. (2013) Integrating trait- and niche-based approaches to assess contemporary evolution in alien plant species. Journal of Ecology, 101, 68-77.

Hurlbert, S.H. (1984) Pseudoreplication and the design of ecological field experiments. Ecological Monographs, 54, 187-211.

Hutchinson, G.E. (1957) Concluding remarks. Cold Spring Harbor Symposia on Quantative Biology, 22, 415-427.

Hyldgaard, B. \& Brix, H. (2012) Intraspecies differences in phenotypic plasticity: Invasive versus non-invasive populations of Ceratophyllum demersum. Aquatic Botany, 97, 4956.

Jackson, S.T. \& Overpeck, J.T. (2000) Responses of plant populations and communities to environmental changes of the late Quaternary. Paleobiology, 26, 194-220.

Jakobs, G., Weber, E. \& Edwards, P.J. (2004) Introduced plants of the invasive Solidago gigantea (Asteraceae) are larger and grow denser than conspecifics in the native range. Diversity and Distributions, 10, 11-19.

Jeschke, J.M. \& Strayer, D.L. (2008) Usefulness of bioclimatic models for studying climate change and invasive species. Annals of the New York Academy of Sciences, 1134, 124. 
Jiménez-Valverde, A. (2012) Insights into the area under the receiver operating characteristic curve (AUC) as a discrimination measure in species distribution modelling. Global Ecology and Biogeography, 21, 498-507.

Joshi, J., Schmid, B., Caldeira, M.C., Dimitrakopoulos, P.G., Good, J., Harris, R., Hector, A., Huss-Danell, K., Jumpponen, A., Minns, A., Mulder, C.P.H., Pereira, J.S., Prinz, A., Scherer-Lorenzen, M., Siamantziouras, A.S.D., Terry, A.C., Troumbis, A.Y. \& Lawton, J.H. (2001) Local adaptation enhances performance of common plant species. Ecology Letters, 4, 536-544.

Kattge, J., Diaz, S., Lavorel, S., Prentice, I.C., Leadley, P., BÖNisch, G., Garnier, E., Westoby, M., Reich, P.B., Wright, I.J., Cornelissen, J.H.C., Violle, C., Harrison, S.P., Van Bodegom, P.M., Reichstein, M., Enquist, B.J., Soudzilovskaia, N.A., Ackerly, D.D., Anand, M., Atkin, O., Bahn, M., Baker, T.R., Baldocchi, D., Bekker, R., Blanco, C.C., Blonder, B., Bond, W.J., Bradstock, R., Bunker, D.E., Casanoves, F., Cavender-Bares, J., Chambers, J.Q., Chapin lii, F.S., Chave, J., Coomes, D., Cornwell, W.K., Craine, J.M., Dobrin, B.H., Duarte, L., Durka, W., Elser, J., Esser, G., Estiarte, M., Fagan, W.F., Fang, J., FernaNdez-Mendez, F., Fidelis, A., Finegan, B., Flores, O., Ford, H., Frank, D., Freschet, G.T., Fyllas, N.M., Gallagher, R.V., Green, W.A., Gutierrez, A.G., Hickler, T., Higgins, S.I., Hodgson, J.G., Jalili, A., Jansen, S., Joly, C.A., Kerkhoff, A.J., Kirkup, D., Kitajima, K., Kleyer, M., Klotz, S., Knops, J.M.H., Kramer, K., Kuhn, I., Kurokawa, H., Laughlin, D., Lee, T.D., Leishman, M., Lens, F., Lenz, T., Lewis, S.L., Lloyd, J., Llusia, J., Louault, F., Ma, S., Mahecha, M.D., Manning, P., Massad, T., Medlyn, B.E., Messier, J., Moles, A.T., Muller, S.C., Nadrowski, K., Naeem, S., Niinemets, Ü., Nollert, S., Nuske, A., Ogaya, R., Oleksyn, J., Onipchenko, V.G., Onoda, Y., Ordonez, J., Overbeck, G., Ozinga, W.A., Patino, S., Paula, S., Pausas, J.G., Penuelas, J., Phillips, O.L., Pillar, V., Poorter, H., Poorter, L., Poschlod, P., Prinzing, A., Proulx, R., Rammig, A., Reinsch, S., Reu, B., Sack, L., Salgado-Negret, B., Sardans, J., Shiodera, S., Shipley, B., Siefert, A., Sosinski, E., Soussana, J.F., Swaine, E., Swenson, N., Thompson, K., Thornton, P., Waldram, M., Weiher, E., White, M., White, S., Wright, S.J., Yguel, B., Zaehle, S., Zanne, A.E. \& Wirth, C. (2011) TRY - a global database of plant traits. Global Change Biology, 17, 2905-2935.

Kaufman, S.R. \& Smouse, P.E. (2001) Comparing indigenous and introduced populations of Melaleuca quinquenervia (Cav.) Blake: response of seedlings to water and $\mathrm{pH}$ levels. Oecologia, 127, 487-494.

Keane, R.M. \& Crawley, M.J. (2002) Exotic plant invasions and the enemy release hypothesis. Trends in Ecology \& Evolution, 17, 164-170.

Kearney, M. \& Porter, W. (2009) Mechanistic niche modelling: combining physiological and spatial data to predict species' ranges. Ecology Letters, 12, 334-350.

Kearney, M.R., Wintle, B.A. \& Porter, W.P. (2010) Correlative and mechanistic models of species distribution provide congruent forecasts under climate change. Conservation Letters, 3, 203-213.

Khan, M.B., Hussain, N. \& Iqbal, M. (2001) Effect of water stress on growth and yield components of maize variety YHS 202. Journal of Research (Science), 12, 15-18. 
Kolbe, J.J., VanMiddlesworth, P.S., Losin, N., Dappen, N. \& Losos, J.B. (2012) Climatic niche shift predicts thermal trait response in one but not both introductions of the Puerto Rican lizard Anolis cristatellus to Miami, Florida, USA. Ecology and Evolution, 2, 15031516.

Kriticos, D.J., Webber, B.L., Leriche, A., Ota, N., Macadam, I., Bathols, J. \& Scott, J.K. (2012) CliMond: global high-resolution historical and future scenario climate surfaces for bioclimatic modelling. Methods in Ecology and Evolution, 3, 53-64.

Kumar, S., LeBrun, E.G., Stohlgren, T.J., Stabach, J.A., McDonald, D.L., Oi, D.H. \& LaPolla, J.S. (2015) Evidence of niche shift and global invasion potential of the Tawny Crazy ant, Nylanderia fulva. Ecology and Evolution, 5, 4628-4641.

Lajeunesse, M.J. (2015) Bias and correction for the log response ratio in ecological metaanalysis. Ecology, 96, 2056-2063.

Lamarque, L.J., Porté, A.J., Eymeric, C., Lasnier, J.-B., Lortie, C.J. \& Delzon, S. (2013) A test for pre-adapted phenotypic plasticity in the invasive tree Acer negundo L. PLOS ONE, 8, e74239.

Lambers, H. \& Poorter, H. (1992) Inherent variation in growth rate between higher plants: a search for physiological causes and ecological consequences. Advances in ecological research, 23, 187-261.

Lambrinos, J.G. (2004) How interactions between ecology and evolution influence contemporary invasion dynamics. Ecology, 85, 2061-2070.

Lande, R. (1980) Genetic variation and phenotypic evolution during allopatric speciation. The American Naturalist, 116, 463-479.

Lande, R. (2009) Adaptation to an extraordinary environment by evolution of phenotypic plasticity and genetic assimilation. Journal of Evolutionary Biology, 22, 1435-1446.

Lande, R. (2015) Evolution of phenotypic plasticity in colonizing species. Molecular Ecology, 24, 2038-2045.

Larcher, W. (2003) Physiological plant ecology; ecophysiology and stress physiology of functional groups. Springer-Verlag, Berlin.

Lavergne, S. \& Molofsky, J. (2007) Increased genetic variation and evolutionary potential drive the success of an invasive grass. Proceedings of the National Academy of Sciences, 104, 3883-3888.

Law, W. \& Salick, J. (2005) Human-induced dwarfing of Himalayan snow lotus, Saussurea laniceps (Asteraceae). Proceedings of the National Academy of Sciences of the United States of America, 102, 10218-10220.

Lawson, A.M. \& Weir, J.T. (2014) Latitudinal gradients in climatic-niche evolution accelerate trait evolution at high latitudes. Ecology Letters, 17, 1427-1436.

Lázaro-Nogal, A., Matesanz, S., Godoy, A., Pérez-Trautman, F., Gianoli, E. \& Valladares, F. (2015) Environmental heterogeneity leads to higher plasticity in dry-edge populations 
of a semi-arid Chilean shrub: insights into climate change responses. Journal of Ecology, 103, 338-350.

Lee, C.E. (2002) Evolutionary genetics of invasive species. Trends in Ecology \& Evolution, 17, 386-391.

Lee, Y.W., Gould, B.A. \& Stinchcombe, J.R. (2014) Identifying the genes underlying quantitative traits: a rationale for the QTN programme. AoB PLANTS, 6, plu004plu004.

Legendre, P. (1993) Spatial autocorrelation: Trouble or new paradigm? Ecology, 74, 16591673.

Leiss, K.A. \& Müller-Schärer, H. (2001) Adaptation of Senecio vulgaris (Asteraceae) to ruderal and agricultural habitats. American Journal of Botany, 88, 1593-1599.

Lennon, J.J. (2000) Red-shifts and red herrings in geographical ecology. Ecography, 23, 101113.

Levin, D.A. (1988) Local differentiation and the breeding structure of plant populations. Plant Evolutionary Biology (ed. by L.D. Gottlieb and S.K. Jain), pp. 305-329. Springer Netherlands, Dordrecht.

Linares, J.C. \& Camarero, J.J. (2012) From pattern to process: linking intrinsic water-use efficiency to drought-induced forest decline. Global Change Biology, 18, 1000-1015.

Lira-Noriega, A. \& Manthey, J.D. (2014) Relationship of genetic diversity and niche centrality: a survey and analysis. Evolution, 68, 1082-1093.

Lobo, J.M., Jiménez-Valverde, A. \& Hortal, J. (2010) The uncertain nature of absences and their importance in species distribution modelling. Ecography, 33, 103-114.

Lortie, C.J. \& Aarssen, L.W. (1996) The specialization hypothesis for phenotypic plasticity in plants. International Journal of Plant Sciences, 157, 484-487.

Mägi, M., Semchenko, M., Kalamees, R. \& Zobel, K. (2011) Limited phenotypic plasticity in range-edge populations: a comparison of co-occurring populations of two Agrimonia species with different geographical distributions. Plant Biology, 13, 177-184.

Mandle, L., Warren, D.L., Hoffmann, M.H., Peterson, A.T., Schmitt, J. \& von Wettberg, E.J. (2011) Conclusions about niche rxpansion in introduced Impatiens walleriana populations depend on method of analysis. PLOS ONE, 5, e15297.

Maron, J.L., Elmendorf, S.C. \& Vilà, M. (2007) Contrasting plant physiological adapation to climate in the native and introduced range of Hypericum perforatum. Evolution, 61, 1912-1924.

Maron, J.L., Vilà, M., Bommarco, R., Elmendorf, S. \& Beardsley, P. (2004) Rapid evolution of an invasive plant. Ecological Monographs, 74, 261-280.

Martínez-Meyer, E., Díaz-Porras, D., Peterson, A.T. \& Yáñez-Arenas, C. (2013) Ecological niche structure and rangewide abundance patterns of species. Biology Letters, 9 
Matute, D.R. (2013) The role of founder effects on the evolution of reproductive isolation. Journal of Evolutionary Biology, 26, 2299-2311.

Matzek, V. (2012) Trait values, not trait plasticity, best explain invasive species' performance in a changing environment. PLOS ONE, 7, e48821.

Maun, M.A. (1998) Adaptations of plants to burial in coastal sand dunes. Canadian Journal of Botany, 76, 713-738.

Maun, M.A. (2009) The biology of coastal sand dunes. Oxford University Press, Oxford.

Maun, M.A., Elberling, H. \& D'Ulisse, A. (1996) The effects of burial by sand on survival and growth of Pitcher's thistle (Cirsium pitcheri) along lake huron. Journal of Coastal Conservation, 2, 3-12.

Maxwell, K. \& Johnson, G.N. (2000) Chlorophyll fluorescence-a practical guide. Journal of Experimental Botany, 51, 659-668.

Mayr, E. (1942) Systematics and the origin of species. Columbia University Press, New York.

Mayr, E. (1954) Change of genetic environment and evolution. Evolution as a process (ed. by J. Huxley, A.C. Hardy and E.B. Ford), pp. 157-180. Allen \& Unwin, London.

McDonald, P.G., Fonseca, C.R., Overton, J.M.C.C. \& Westoby, M. (2003) Leaf-size divergence along rainfall and soil-nutrient gradients: is the method of size reduction common among clades? Functional Ecology, 17, 50-57.

McDowell, S.C.L. (2002) Photosynthetic characteristics of invasive and noninvasive species of Rubus (Rosaceae). American Journal of Botany, 89, 1431-1438.

McGill, B. (2015) Why AIC appeals to ecologist's lowest instincts. Available at https://dynamicecology.wordpress.com/ (accessed 11 February 2017).

McGraw, J.B. (2001) Evidence for decline in stature of American ginseng plants from herbarium specimens. Biological Conservation, 98, 25-32.

McLellan, T. \& Endler, J.A. (1998) The relative success of some methods for measuring and describing the shape of complex objects. Systematic Biology, 47, 264-281.

McWilliams, S.R., Afik, D. \& Secor, S. (1997) Patterns and processes in the vertebrate digestive system. Trends in Ecology \& Evolution, 12, 420-422.

Medley, K.A. (2010) Niche shifts during the global invasion of the Asian tiger mosquito, Aedes albopictus Skuse (Culicidae), revealed by reciprocal distribution models. Global Ecology and Biogeography, 19, 122-133.

Medrano, H., Flexas, J. \& Galmés, J. (2009) Variability in water use efficiency at the leaf level among Mediterranean plants with different growth forms. Plant and Soil, 317, 17-29.

Meyerson, L.A. \& Mooney, H.A. (2007) Invasive alien species in an era of globalization. Frontiers in Ecology and the Environment, 5, 199-208. 
Miller-Rushing, A.J., Primack, R.B., Primack, D. \& Mukunda, S. (2006) Photographs and herbarium specimens as tools to document phenological changes in response to global warming. American Journal of Botany, 93, 1667-1674.

Moles, A.T., Warton, D.I., Warman, L., Swenson, N.G., Laffan, S.W., Zanne, A.E., Pitman, A., Hemmings, F.A. \& Leishman, M.R. (2009) Global patterns in plant height. Journal of Ecology, 97, 923-932.

Moles, A.T., Perkins, S.E., Laffan, S.W., Flores-Moreno, H., Awasthy, M., Tindall, M.L., Sack, L., Pitman, A., Kattge, J. \& Aarssen, L.W. (2014) Which is a better predictor of plant traits: temperature or precipitation? Journal of Vegetation Science, 25, 1167-1180.

Molina-Montenegro, M.A., Salgado-Luarte, C., Oses, R. \& Torres-Díaz, C. (2013) Is physiological performance a good predictor for ftness? Insights from an invasive plant species. PLOS ONE, 8, e76432.

Moody, M.E. \& Mack, R.N. (1988) Controlling the spread of plant invasions: The importance of nascent foci. Journal of Applied Ecology, 25, 1009-1021.

Mooney, H.A. \& Cleland, E.E. (2001) The evolutionary impact of invasive species. Proceedings of the National Academy of Sciences, 98, 5446-5451.

Moran, N.A. (1992) The evolutionary maintenance of alternative phenotypes. The American Naturalist, 139, 971-989.

Müller-Schärer, H. \& Steinger, T. (2004) Predicting evolutionary change in invasive, exotic plants and its consequences for plant-herbivore interactions. Genetics, evolution and biological control (ed. by L.E. Ehler, R. Sforza and T. Mateille), pp. 137-162. CABI, Wallingford, UK.

Müller-Schärer, H., Schaffner, U. \& Steinger, T. (2004) Evolution in invasive plants: implications for biological control. Trends in Ecology \& Evolution, 19, 417-422.

Munné-Bosch, S. \& Alegre, L. (2004) Die and let live: leaf senescence contributes to plant survival under drought stress. Functional Plant Biology, 31, 203-216.

Nakagawa, S. \& Cuthill, I.C. (2007) Effect size, confidence interval and statistical significance: a practical guide for biologists. Biological Reviews, 82, 591-605.

Navas, M.-L. \& Garnier, E. (2002) Plasticity of whole plant and leaf traits in Rubia peregrina in response to light, nutrient and water availability. Acta Oecologica, 23, 375-383.

Nicotra, A.B., Leigh, A., Boyce, C.K., Jones, C.S., Niklas, K.J., Royer, D.L. \& Tsukaya, H. (2011) The evolution and functional significance of leaf shape in the angiosperms. Functional Plant Biology, 38, 535-552.

Nicotra, A.B., Atkin, O.K., Bonser, S.P., Davidson, A.M., Finnegan, E.J., Mathesius, U., Poot, P., Purugganan, M.D., Richards, C.L., Valladares, F. \& van Kleunen, M. (2010) Plant phenotypic plasticity in a changing climate. Trends in Plant Science, 15, 684-692. 
Nieminen, P., Lehtiniemi, H., Vähäkangas, K., Huusko, A. \& Rautio, A. (2013) Standardised regression coefficient as an effect size index in summarising findings in epidemiological studies. Epidemiology, Biostatistics and Public Health, 10

Niinemets, Ü. (2001) Global-scale climate controls of leaf dry mass per area, density, and thickness in trees and shrubs. Ecology, 82, 453-469.

Novak, S.J. (2007) The role of evolution in the invasion process. Proceedings of the National Academy of Sciences, 104, 3671-3672.

Padilla, D.K. \& Adolph, S.C. (1996) Plastic inducible morphologies are not always adaptive: The importance of time delays in a stochastic environment. Evolutionary Ecology, 10, 105-117.

Palacio-López, K. \& Gianoli, E. (2011) Invasive plants do not display greater phenotypic plasticity than their native or non-invasive counterparts: a meta-analysis. Oikos, 120, 1393-1401.

Pamela J. Yeh \& Trevor D. Price (2004) Adaptive phenotypic plasticity and the successful colonization of a novel environment. The American Naturalist, 164, 531-542.

Parker, I.M., Rodriguez, J. \& Loik, M.E. (2003) An evolutionary approach to understanding the biology of invasions: local adaptation and general-purpose genotypes in the weed Verbascum thapsus. Conservation Biology, 17, 59-72.

Parkhurst, D.F. \& Loucks, O.L. (1972) Optimal leaf size in relation to environment. Journal of Ecology, 60, 505-537.

Pearman, P.B., Randin, C.F., Broennimann, O., Vittoz, P., Knaap, W.O.v.d., Engler, R., Lay, G.L., Zimmermann, N.E. \& Guisan, A. (2008) Prediction of plant species distributions across six millennia. Ecology Letters, 11, 357-369.

Pearson, R.G. \& Dawson, T.P. (2003) Predicting the impacts of climate change on the distribution of species: are bioclimate envelope models useful? Global Ecology and Biogeography, 12, 361-371.

Pelletier, F., Garant, D., \& Hendry, A. P. (2009). Introduction: Eco-Evolutionary dynamics. Philosophical Transactions: Biological Sciences, 364, 1483-1489.

Peppe, D.J., Royer, D.L., Cariglino, B., Oliver, S.Y., Newman, S., Leight, E., Enikolopov, G., Fernandez-Burgos, M., Herrera, F., Adams, J.M., Correa, E., Currano, E.D., Erickson, J.M., Hinojosa, L.F., Hoganson, J.W., Iglesias, A., Jaramillo, C.A., Johnson, K.R., Jordan, G.J., Kraft, N.J.B., Lovelock, E.C., Lusk, C.H., Niinemets, Ü., Peñuelas, J., Rapson, G., Wing, S.L. \& Wright, I.J. (2011) Sensitivity of leaf size and shape to climate: global patterns and paleoclimatic applications. New Phytologist, 190, 724-739.

Pérez-Harguindeguy, N., Díaz, S., Garnier, E., Lavorel, S., Poorter, H., Jaureguiberry, P., BretHarte, M.S., Cornwell, W.K., Craine, J.M., Gurvich, D.E., Urcelay, C., Veneklaas, E.J., Reich, P.B., Poorter, L., Wright, I.J., Ray, P., Enrico, L., Pausas, J.G., de Vos, A.C., Buchmann, N., Funes, G., Quétier, F., Hodgson, J.G., Thompson, K., Morgan, H.D., ter Steege, H., Sack, L., Blonder, B., Poschlod, P., Vaieretti, M.V., Conti, G., Staver, A.C., Aquino, S. \& Cornelissen, J.H.C. (2013) New handbook for standardised 
measurement of plant functional traits worldwide. Australian Journal of Botany, 61, 167-234.

Pergams, O.R.W. \& Ashley, M.V. (2001) Microevolution in island rodents. Genetica, 112, 245256.

Perrings, C., Burgiel, S., Lonsdale, M., Mooney, H.A. \& Williamson, M. (2010) Globalization and bioinvasions: The international policy problem. Bioinvasions and Globalization: Ecology, Economics, Management and Policy (ed. by C. Perrings and H.A. Mooney), pp. 235-249. Oxford University Press, Oxford, UK.

Peterson, A.T. (2003) Predicting the geography of species' invasions via ecological niche modeling. The Quarterly Review of Biology, 78, 419-433.

Peterson, A.T. (2006) Ecologic niche modeling and spatial patterns of disease transmission. Emerging Infectious Diseases, 12, 1822-1826.

Peterson, A.T. (2007) Ecological niche modelling and understanding the geography of disease transmission. Veterinaria italiana, 43, 393-400.

Peterson, A.T. (2011) Ecological niche conservatism: a time-structured review of evidence. Journal of Biogeography, 38, 817-827.

Peterson, A.T. \& Vieglais, D.A. (2001) Predicting species invasions using ecological niche modeling: New approaches from bioinformatics atack a Pressing Problem. BioScience, 51, 363-371.

Petitpierre, B., Kueffer, C., Broennimann, O., Randin, C., Daehler, C. \& Guisan, A. (2012) Climatic niche shifts are rare among terrestrial plant invaders. Science, 335, 13441348.

Pfennig, D.W. \& McGee, M. (2010) Resource polyphenism increases species richness: a test of the hypothesis. Philosophical Transactions of the Royal Society B: Biological Sciences, 365, 577-591.

Phillips, B.L., Brown, G.P., Webb, J.K. \& Shine, R. (2006) Invasion and the evolution of speed in toads. Nature, $439,803-803$.

Pigliucci, M. (2001) Phenotypic plastity, beyond nature and nuture. Johns Hopkins University press, Baltimore.

Pigliucci, M. (2005) Evolution of phenotypic plasticity: where are we going now? Trends in Ecology \& Evolution, 20, 481-486.

Pigliucci, M., Murren, C.J. \& Schlichting, C.D. (2006) Phenotypic plasticity and evolution by genetic assimilation. Journal of Experimental Biology, 209, 2362-2367.

Poorter, H. \& Nagel, O. (2000) The role of biomass allocation in the growth response of plants to different levels of light, $\mathrm{CO}_{2}$, nutrients and water: a quantitative review. Functional Plant Biology, 27, 595-607. 
Poorter, H., Niinemets, Ü., Poorter, L., Wright, I.J. \& Villar, R. (2009) Causes and consequences of variation in leaf mass per area (LMA): a meta-analysis. New Phytologist, 182, 565-588.

Poorter, H., Niinemets, Ü., Walter, A., Fiorani, F. \& Schurr, U. (2010) A method to construct dose-response curves for a wide range of environmental factors and plant traits by means of a meta-analysis of phenotypic data. Journal of Experimental Botany, 61, 2043-2055.

Poorter, H., Niklas, K.J., Reich, P.B., Oleksyn, J., Poot, P. \& Mommer, L. (2012) Biomass allocation to leaves, stems and roots: meta-analyses of interspecific variation and environmental control. New Phytologist, 193, 30-50.

Porfirio, L.L., Harris, R.M.B., Lefroy, E.C., Hugh, S., Gould, S.F., Lee, G., Bindoff, N.L. \& Mackey, B. (2014) Improving the use of species distribution models in conservation planning and management under climate change. PLOS ONE, 9, e113749.

Porter, J.R. \& Semenov, M.A. (2005) Crop responses to climatic variation. Philosophical Transactions of the Royal Society B: Biological Sciences, 360, 2021-2035.

Prentis, P.J., Wilson, J.R.U., Dormontt, E.E., Richardson, D.M. \& Lowe, A.J. (2008) Adaptive evolution in invasive species. Trends in Plant Science, 13, 288-294.

Pyšek, P. \& Richardson, D.M. (2007) Traits associated with invasiveness in alien plants: Where do we stand? Biological Invasions (ed. by W. Nentwig), pp. 97-125. SpringerVerlag, Berlin, Heidelberg.

R Core Team (2013) R: A language and environment for statistical computing. $R$ Foundation for Statistical Computing. Vienna, Austria. Available at https://www.r-project.org/.

Randall, R.P. (2012) A global compendium of weeds, Second edn. Department of Agriculture and Food, Western Australia.

Rausher, M.D. \& Delph, L.F. (2015) Commentary: When does understanding phenotypic evolution require identification of the underlying genes? Evolution, 69, 1655-1664.

Reich, P.B., Walters, M.B. \& Ellsworth, D.S. (1997) From tropics to tundra: Global convergence in plant functioning. Proceedings of the National Academy of Sciences, 94, $13730-13734$.

Reznick, D.N. \& Ghalambor, C.K. (2001) The population ecology of contemporary adaptations: what empirical studies reveal about the conditions that promote adaptive evolution. Genetica, 112, 183-198.

Richards, C.L., Bossdorf, O., Muth, N.Z., Gurevitch, J. \& Pigliucci, M. (2006) Jack of all trades, master of some? On the role of phenotypic plasticity in plant invasions. Ecology Letters, 9, 981-993.

Robinson, B.W. \& Wilson, D.S. (1994) Character release and displacement in fishes: A neglected literature. The American Naturalist, 144, 596-627. 
Rödder, D. \& Lötters, S. (2009) Niche shift versus niche conservatism? Climatic characteristics of the native and invasive ranges of the Mediterranean house gecko (Hemidactylus turcicus). Global Ecology and Biogeography, 18, 674-687.

Rollins, L.A., Moles, A.T., Lam, S., Buitenwerf, R., Buswell, J.M., Brandenburger, C.R., FloresMoreno, H., Nielsen, K.B., Couchman, E., Brown, G.S., Thomson, F.J., Hemmings, F., Frankham, R. \& Sherwin, W.B. (2013) High genetic diversity is not essential for successful introduction. Ecology and Evolution, 3, 4501-4517.

Rosenthal, R. (1979) The file drawer problem and tolerance for null results. Psychological Bulletin, 86, 638-641.

Rucker, K.S., Kvien, C.K., Holbrook, C.C. \& Hook, J.E. (1995) Identification of peanut genotypes with improved drought avoidance traits. Peanut Science, 22, 14-18.

Ryser, P. \& Eek, L. (2000) Consequences of phenotypic plasticity vs. interspecific differences in leaf and root traits for acquisition of aboveground and belowground resources. American Journal of Botany, 87, 402-411.

Sakai, A.K., Allendorf, F.W., Holt, J.S., Lodge, D.M., Molofsky, J., With, K.A., Baughman, S., Cabin, R.J., Cohen, J.E. \& Ellstrand, N.C. (2001) The population biology of invasive species. Annual review of ecology and systematics, 32, 305-332.

Samsone, I., Druva-Lūsīte, I., Andersone, U., N̦ečajeva, J., Karlsons, A. \& levinsh, G. Plasticity of a dune plant Alyssum gmelinii in response to sand burial in natural conditions.

Sarkar, S. (2004) From the Reaktionsnorm to the evolution of adaptive plasticity. Phenotypic plasticity. Functional and conceptual approaches (ed. by T.J. Dewitt and S.M. Scheiner), pp. 10-30. New York, Oxford University Press.

Sax, D.F., Stachowicz, J.J., Brown, J.H., Bruno, J.F., Dawson, M.N., Gaines, S.D., Grosberg, R.K., Hastings, A., Holt, R.D., Mayfield, M.M., O'Connor, M.I. \& Rice, W.R. (2007) Ecological and evolutionary insights from species invasions. Trends in Ecology \& Evolution, 22, 465-471.

Scheiner, S.M. (1993) Genetics and evolution of phenotypic plasticity. Annual review of ecology and systematics, 24, 35-68.

Scheiner, S.M. \& Berrigan, D. (1998) The genetics of phenotypic plasticity. VIII. the cost of plasticity in Daphnia pulex. Evolution, 52, 368-378.

Schlichting, C.D. (1986) The evolution of phenotypic plasticity in plants. Annual review of ecology and systematics, 17, 667-693.

Schlichting, C.D. (2002) Phenotypic plasticity in plants. Plant Species Biology, 17, 85-88.

Schlichting, C.D. (2004) The role of phenotypic plasticity in diversification. Phenotypic Plasticity: Functional and Conceptual Approaches (ed. by T.J. Dewitt and S.M. Scheiner), pp. 191-200. Oxford University Press, Oxford.

Schlichting, C.D. \& Pigliucci, M. (1998) Phenotypic evolution: a reaction norm perspective. Sinauer Associates Incorporated, Sunderland, USA. 
Schlichting, C.D. \& Smith, H. (2002) Phenotypic plasticity: linking molecular mechanisms with evolutionary outcomes. Evolutionary Ecology, 16, 189-211.

Schneider, C.A., Rasband, W.S. \& Eliceiri, K.W. (2012) NIH Image to ImageJ: 25 years of image analysis. Nat Meth, 9, 671-675.

Seibt, U., Rajabi, A., Griffiths, H. \& Berry, J.A. (2008) Carbon isotopes and water use efficiency: sense and sensitivity. Oecologia, 155, 441.

Senay, S.D., Worner, S.P. \& Ikeda, T. (2013) Novel three-step pseudo-absence selection technique for improved species distribution modelling. PLOS ONE, 8, e71218.

Sexton, J.P., McKay, J.K. \& Sala, A. (2002) Plasticity and genetic diversity may allow saltcedar to invade cold climates in north america. Ecological Applications, 12, 1652-1660.

Shi, L., Zhang, Z.J., Zhang, C.Y. \& Zhang, J.Z. (2004) Effects of sand burial on survival, growth, gas exchange and biomass allocation of Ulmus pumila seedlings in the Hunshandak Sandland, China. Annals of Botany, 94, 553-560.

Silvertown, J. (2004) Plant coexistence and the niche. Trends in Ecology \& Evolution, 19, 605611.

Simberloff, D., Dayan, T., Jones, C. \& Ogura, G. (2000) Character displacement and release in the small indian mongoose, Herpestes javanicus. Ecology, 81, 2086-2099.

Smekens, M.J. \& van Tienderen, P.H. (2001) Genetic variation and plasticity of Plantago coronopus under saline conditions. Acta Oecologica, 22, 187-200.

Smith, T.B. (1993) Disruptive selection and the genetic basis of bill size polymorphism in the African finch Pyrenestes. Nature, 363, 618-620.

Soberón, J. \& Peterson, A.T. (2011) Ecological niche shifts and environmental space anisotropy: a cautionary note. Revista Mexicana de Biodiversidad, 82, 1348-1355.

Stapley, J., Santure, A.W. \& Dennis, S.R. (2015) Transposable elements as agents of rapid adaptation may explain the genetic paradox of invasive species. Molecular Ecology, 24, 2241-2252.

Stearns, S.C. (1982) The role of development in the evolution of life histories. Evolution and Development: Report of the Dahlem Workshop on Evolution and Development Berlin (ed. by J.T. Bonner), pp. 237-258. Springer, Berlin, Heidelberg.

Stearns, S.C. (1989) The evolutionary significance of phenotypic plasticity. BioScience, 39, 436-445.

Stevens, C.J., Dupré, C., Dorland, E., Gaudnik, C., Gowing, D.J.G., Diekmann, M., Alard, D., Bobbink, R., Corcket, E., Mountford, J.O., Vandvik, V., Aarrestad, P.A., Muller, S. \& Dise, N.B. (2011) Grassland species composition and biogeochemistry in 153 sites along environmental gradients in Europe. Ecology, 92, 1544-1544.

Still, D.W., Kim, D.H. \& Aoyama, N. (2005) Genetic variation in Echinacea angustifolia along a climatic gradient. Annals of Botany, 96, 467-477. 
Stockwell, C.A. \& Ashley, M.V. (2004) Rapid adaptation and conservation. Conservation Biology, 18, 272-273.

Stockwell, C.A., Hendry, A.P. \& Kinnison, M.T. (2003) Contemporary evolution meets conservation biology. Trends in Ecology \& Evolution, 18, 94-101.

Stomp, M., van Dijk, Mark A., van Overzee, Harriët M.J., Wortel, Meike T., Sigon, Corrien A.M., Egas, M., Hoogveld, H., Gons, Herman J. \& Huisman, J. (2008) The timescale of phenotypic plasticity and Its impact on competition in fluctuating environments. The American Naturalist, 172, E169-E185.

Strubbe, D., Beauchard, O. \& Matthysen, E. (2015) Niche conservatism among non-native vertebrates in Europe and North America. Ecography, 38, 321-329.

Suarez, A.V. \& Tsutsui, N.D. (2008) The evolutionary consequences of biological invasions. Molecular Ecology, 17, 351-360.

Sultan, S.E. (1987) Evolutionary implications of phenotypic plasticity in plants. Evolutionary Biology: Volume 21 (ed. by M.K. Hecht, B. Wallace and G.T. Prance), pp. 127-178. Springer US, Boston, MA.

Sultan, S.E. (2001) Phenotypic plasticity for fitness components in Polygonum species of contrasting ecological breadth. Ecology, 82, 328-343.

Sultan, S.E. \& Bazzaz, F.A. (1993) Phenotypic plasticity in Polygonum persicaria. III. The evolution of ecological breadth for nutrient environment. Evolution, 47, 1050-1071.

Svanbäck, R. \& Schluter, D. (2012) Niche specialization influences adaptive phenotypic plasticity in the threespine stickleback. The American Naturalist, 180, 50-59.

Svanbäck, R., Pineda-Krch, M. \& Doebeli, M. (2009) Fluctuating population dynamics promotes the evolution of phenotypic plasticity. The American Naturalist, 174, 176189.

Taylor, D.R. \& Aarssen, L.W. (1988) An interpretation of phenotypic plasticity in Agropyron repens (Graminae). American Journal of Botany, 75, 401-413.

Thébaud, C. \& Simberloff, D. (2001) Are plants really larger in their introduced ranges? The American Naturalist, 157, 231-236.

Thompson, J.D. (1991) Phenotypic plasticity as a component of evolutionary change. Trends in Ecology \& Evolution, 6, 246-249.

Thompson, J.N. (1998) Rapid evolution as an ecological process. Trends in Ecology \& Evolution, 13, 329-332.

Thuiller, W., Lavorel, S., Araújo, M.B., Sykes, M.T. \& Prentice, I.C. (2005a) Climate change threats to plant diversity in Europe. Proceedings of the National Academy of Sciences of the United States of America, 102, 8245-8250.

Thuiller, W., Richardson, D.M., Pyšek, P., Midgley, G.F., Hughes, G.O. \& Rouget, M. (2005b) Niche-based modelling as a tool for predicting the risk of alien plant invasions at a global scale. Global Change Biology, 11, 2234-2250. 
Thuiller, W., Albert, C.H., Dubuis, A., Randin, C. \& Guisan, A. (2009) Variation in habitat suitability does not always relate to variation in species' plant functional traits. Biology Letters, 6, rsbl20090669.

Tingley, R., Vallinoto, M., Sequeira, F. \& Kearney, M.R. (2014) Realized niche shift during a global biological invasion. Proceedings of the National Academy of Sciences, 111, 10233-10238.

Tingley, R., Thompson, M.B., Hartley, S. \& Chapple, D.G. (2016) Patterns of niche filling and expansion across the invaded ranges of an Australian lizard. Ecography, 39, 270-280.

Tufto, J. (2000) The evolution of plasticity and nonplastic spatial and temporal adaptations in the presence of imperfect environmental cues. The American Naturalist, 156, 121-130.

Turner, K.G., Hufbauer, R.A. \& Rieseberg, L.H. (2014) Rapid evolution of an invasive weed. New Phytologist, 202, 309-321.

Václavík, T., Beckmann, M., Cord, A.F. \& Bindewald, A.M. (2017) Effects of UV-B radiation on leaf hair traits of invasive plants-Combining historical herbarium records with novel remote sensing data. PLOS ONE, 12, e0175671.

Vadillo, M.A., Konstantinidis, E. \& Shanks, D.R. (2016) Underpowered samples, false negatives, and unconscious learning. Psychonomic Bulletin \& Review, 23, 87-102.

Valladares, F., Sanchez-Gomez, D. \& Zavala, M.A. (2006) Quantitative estimation of phenotypic plasticity: bridging the gap between the evolutionary concept and its ecological applications. Journal of Ecology, 94, 1103-1116.

van Kleunen, M. \& Schmid, B. (2003) No evidence for an evolutionary increased compertative ability in an invasive plant. Ecology, 84, 2816-2823.

van Kleunen, M. \& Fischer, M. (2005) Constraints on the evolution of adaptive phenotypic plasticity in plants. New Phytologist, 166, 49-60.

van Kleunen, M., Fischer, M. \& Schmid, B. (2000) Costs of plasticity in foraging characteristics of the clonal plant Ranunculus reptans. Evolution, 54, 1947-1955.

van Kleunen, M., Schlaepfer, D.R., Glaettli, M. \& Fischer, M. (2011) Preadapted for invasiveness: do species traits or their plastic response to shading differ between invasive and non-invasive plant species in their native range? Journal of Biogeography, 38, 1294-1304.

Van Valen, L. (1965) Morphological variation and width of ecological niche. The American Naturalist, 99, 377-390.

Vaughan, I.P. \& Ormerod, S.J. (2003) Improving the quality of distribution models for conservation by addressing shortcomings in the field collection of training data. Conservation Biology, 17, 1601-1611.

Venables, W.N. \& Ripley, B.D. (2002) Mordern applied statistics with S, Fourth edn. Springer, New York. 
Via, S. (1984) The quantitative genetics of polyphagy in an insect herbivore. I. Genotypeenvironment interaction in larval performance on different host plant species. Evolution, 38, 881-895.

Via, S., Gomulkiewicz, R., De Jong, G., Scheiner, S.M., Schlichting, C.D. \& Van Tienderen, P.H. (1995) Adaptive phenotypic plasticity: consensus and controversy. Trends in Ecology \& Evolution, 10, 212-217.

Villemant, C., Barbet-Massin, M., Perrard, A., Muller, F., Gargominy, O., Jiguet, F. \& Rome, Q. (2011) Predicting the invasion risk by the alien bee-hawking Yellow-legged hornet Vespa velutina nigrithorax across Europe and other continents with niche models. Biological Conservation, 144, 2142-2150.

Violle, C., Navas, M.-L., Vile, D., Kazakou, E., Fortunel, C., Hummel, I. \& Garnier, E. (2007) Let the concept of trait be functional! Oikos, 116, 882-892.

Visser, M.E. (2008) Keeping up with a warming world; assessing the rate of adaptation to climate change. Proceedings of the Royal Society B: Biological Sciences, 275, 649659.

Waddington, C.H. (1953) Genetic assimilation of an acquired character. Evolution, 7, 118-126.

Walker, M.D., Wahren, C.H., Hollister, R.D., Henry, G.H., Ahlquist, L.E., Alatalo, J.M., BretHarte, M.S., Calef, M.P., Callaghan, T.V. \& Carroll, A.B. (2006) Plant community responses to experimental warming across the tundra biome. Proceedings of the National Academy of Sciences, 103, 1342-1346.

Warren, D.L., Glor, R.E. \& Turelli, M. (2008) Environmental niche equivalency versus conservatism: quantative appraoches to niche evolution. Evolution, 62, 2868-2883.

Weinig, C. (2000) Plasticity versus canalization: population differences in the timing of shadeavoidance responses. Evolution, 54, 441-451.

West-Eberhard, M.J. (1989) Phenotypic plasticity and the origins of diversity. Annual review of Ecology and Systematics, 20, 249-278.

West-Eberhard, M.J. (2003) Developmental plasticity and evolution. Oxford University Press, Oxford.

Westoby, M. (1998) A leaf-height-seed (LHS) plant ecology strategy scheme. Plant and Soil, 199, 213-227.

Westoby, M., Falster, D.S., Moles, A.T., Vesk, P.A. \& Wright, I.J. (2002) Plant ecological strategies: some leading dimensions of variation between species. Annual review of ecology and systematics, 33, 125-159.

Whitlock, M.C. (1996) The Red Queen Beats the Jack-Of-All-Trades: The Limitations on the Evolution of Phenotypic Plasticity and Niche Breadth. The American Naturalist, 148, S65-S77.

Whitman, D. \& Agrawal, A. (2009) What is phenotypic plasticity and why is it important? Phenotypic Plasticity of Insects. Science Publishers. 
Whitney, K.D. \& Gabler, C.A. (2008) Rapid evolution in introduced species, 'invasive traits' and recipient communities: challenges for predicting invasive potential. Diversity and Distributions, 14, 569-580.

Wiens, J.J. \& Graham, C.H. (2005) Niche conservatism: integrating evolution, ecology, and conservation biology. Annu. Rev. Ecol. Evol. Syst., 36, 519-539.

Williams, D.G., Mack, R.N. \& Black, R.A. (1995) Ecophysiology of introduced Pennisetum Setaceum on Hawaii: The role of phenotypic plasticity. Ecology, 76, 1569-1580.

Williams, J.L., Auge, H. \& Maron, J.L. (2008) Different gardens, different results: native and introduced populations exhibit contrasting phenotypes across common gardens. Oecologia, 157, 239-248.

Wilson, J.B. \& Sykes, M.T. (1999) Is zonation on coastal sand dunes determined primarily by sand burial or by salt spray? A test in New Zealand dunes. Ecology Letters, 2, 233236.

Wiser, S.K., Bellingham, P.J. \& Burrows, L.E. (2001) Managing biodiversity information: development of New Zealand's National Vegetation Survey databank. New Zealand Journal of Ecology, 25, 1-17.

Wittmann, M.E., Barnes, M.A., Jerde, C.L., Jones, L.A. \& Lodge, D.M. (2016) Confronting species distribution model predictions with species functional traits. Ecology and Evolution, 6, 873-879.

Woltereck, R. (1909) Weitere experimentelle Untersuchungen über Artveränderung, speziel über das Wesen quantitativer Artunterschiede bei Daphniden. Verhandlungen der Deutschen Zoologischen Gesellschaft, 19, 110-173.

Wright, I.J., Reich, P.B. \& Westoby, M. (2001) Strategy shifts in leaf physiology, structure and nutrient content between species of high- and low-rainfall and high- and low-nutrient habitats. Functional Ecology, 15, 423-434.

Wright, I.J., Reich, P.B., Westoby, M., Ackerly, D.D., Baruch, Z., Bongers, F., Cavender-Bares, J., Chapin, T., Cornelissen, J.H.C., Diemer, M., Flexas, J., Garnier, E., Groom, P.K., Gulias, J., Hikosaka, K., Lamont, B.B., Lee, T., Lee, W., Lusk, C., Midgley, J.J., Navas, M.-L., Niinemets, U., Oleksyn, J., Osada, N., Poorter, H., Poot, P., Prior, L., Pyankov, V.I., Roumet, C., Thomas, S.C., Tjoelker, M.G., Veneklaas, E.J. \& Villar, R. (2004) The worldwide leaf economics spectrum. Nature, 428, 821-827.

Wund, M.A. (2012) Assessing the impacts of phenotypic plasticity on evolution. Integrative and Comparative Biology, 52, 5-15.

Xu, Z. \& Zhou, G. (2008) Responses of leaf stomatal density to water status and its relationship with photosynthesis in a grass. Journal of experimental botany, 59, 3317-3325.

Yates, M.J., Anthony Verboom, G., Rebelo, A.G. \& Cramer, M.D. (2010) Ecophysiological significance of leaf size variation in Proteaceae from the Cape Floristic Region. Functional Ecology, 24, 485-492. 
Zenni, R.D., Bailey, J.K. \& Simberloff, D. (2014) Rapid evolution and range expansion of an invasive plant are driven by provenance-environment interactions. Ecology Letters, 17, 727-735.

Zou, J., Rogers, W.E. \& Siemann, E. (2007) Differences in morphological and physiological traits between native and invasive populations of Sapium sebiferum. Functional Ecology, 21, 721-730.

Zou, J., Rogers, W.E. \& Siemann, E. (2009) Plasticity of Sapium sebiferum seedling growth to light and water resources: Inter- and intraspecific comparisons. Basic and Applied Ecology, 10, 79-88. 